Verena Mertins

Institutionen-

ökonomische

Analyse von

Innovationsförderung 
Verena Mertins

\section{Institutionenökonomische Analyse von Innovationsförderung}

Die Unterstützung der Innovationstätigkeit von Unternehmen durch staatliche Förderung stellt ein populäres Instrument der Politik dar. Diese Arbeit untersucht unter Anwendung von Ansätzen der Neuen Institutionenökonomik und Entrepreneurship-Theorie die Ausgestaltung von Innovationsförderung am Beispiel des Niedersächsischen Innovationsförderprogramms und die von diesem Programm ausgehende Wirkung auf die beteiligten Akteure. Daran schließt sich die Darstellung der Ergebnisse einer Unternehmensbefragung an, in welcher die theoretischen und empirischen Erkenntnisse verknüpft werden. Im Mittelpunkt steht dabei die Fragestellung, inwiefern sich Unternehmen, die Innovationsfördermittel erhalten haben, von nicht geförderten Unternehmen unterscheiden.

Verena Mertins wurde 1980 in Hamburg geboren. Sie studierte von 1999 bis 2004 Volkswirtschaftslehre in Göttingen und Poitiers (Frankreich) und arbeitete im Anschluss als Wissenschaftliche Mitarbeiterin an der Universität Göttingen. Ihr Promotionsstudium schloss sie 2008 ab. 
Institutionenökonomische Analyse von Innovationsförderung 


\title{
cege-Schriften
}

Centrum für Europa-, Governance- und Entwicklungsforschung (cege)

Georg-August-Universitöt Göttingen

Center for European, Governance and Economic Development Research (cege)

\section{Band 17}

\author{
Herausgegeben von \\ Kilian Bizer, Stephan von Cramon-Taubadel, \\ Andreas Oestreicher, Renate Ohr, \\ Gerhard Rübel, Robert Schwager, \\ Verantwortlicher Herausgeber für diesen Band: \\ Kilian Bizer
}

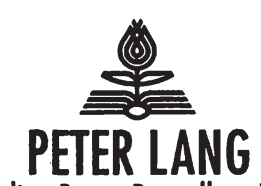

Frankfurt am Main - Berlin - Bern - Bruxelles - New York - Oxford - Wien 


\section{Verena Mertins}

\section{Institutionenökonomische Analyse von Innovationsförderung}

Eine theoretische und empirische Betrachtung am Beispiel Niedersachsens

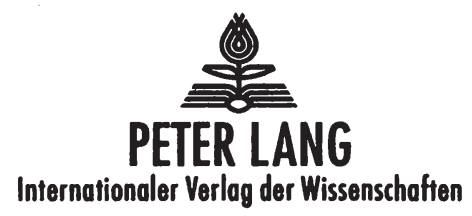


Bibliografische Information der Deutschen Nationalbibliothek Die Deutsche Nationalbibliothek verzeichnet diese Publikation in der Deutschen Nationalbibliografie; detaillierte bibliografische Daten sind im Internet über <http://www.d-nb.de> abrufbar.

Zugl.: Göttingen, Univ., Diss., 2008

Open Access: The online version of this publication is published on www.peterlang.com and www.econstor.eu under the international Creative Commons License CC-BY 4.0. Learn more on how you can use and share this work: http://creativecommons.org/licenses/ by/4.0.

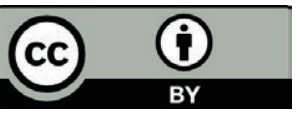

This book is available Open Access thanks to the kind support of ZBW - Leibniz-Informationszentrum Wirtschaft.

Gedruckt auf alterungsbeståndigem, säurefreiem Papier.

\author{
D 7 \\ ISSN 1617-741X \\ ISBN 978-3-631-59185-7 \\ ISBN 978-3-631-75685-0 (eBook) \\ (c) Peter Lang GmbH \\ Internationaler Verlag der Wissenschaften \\ Frankfurt am Main 2009 \\ Alle Rechte vorbehalten.
}

Das Werk einschließlich aller seiner Teile ist urheberrechtlich geschützt. Jede Verwertung außerhalb der engen Grenzen des

Urheberrechtsgesetzes ist ohne Zustimmung des Verlages unzulăssig und strafbar. Das gilt insbesondere für

Vervielfältigungen, Übersetzungen, Mikroverfilmungen und die Einspeicherung und Verarbeitung in elektronischen Systemen.

Printed in Germany 123457

www.peterlang.de 


\section{Vorwort des Herausgebers}

Innovationen können auf vielfältige Weise durch den Staat gefördert werden. Unter der Annahme, dass eine Förderung grundsätzlich sinnvoll ist, weil externe Effekte bestehen, stellt sich die Frage, welche Form der Förderung sinnvoll ist. So gibt es zahlreiche Arbeiten über Patente zum Schutz geistigen Eigentums, die letztlich eine Internalisierung der positiven externen Effekte anstreben. Ebenfalls eine reichhaltige Literatur misst den Ertrag von Innovationsförderung in Form von Arbeitsplätzen und Patenten in Abhängigkeit verschiedener Förderinstrumente. Diese Arbeit geht einen anderen Weg, in dem sie ausgehend von der Neuen Institutionenökonomik und der Unternehmertheorie versucht herauszufinden, ob denn Innovationsförderprogramme die „richtigen“ Unternehmer erreicht. Insofern leistet sie sowohl einen - theoretischen - Beitrag zur Präzisierung der Neuen Institutionenökonomik und Unternehmenstheorie als auch einen empirischen Beitrag zur Wirkungsforschung von Innovationsförderprogrammen, insbesondere des Niedersächsischen Innovationsförderprogramms.

Für die Ausgestaltung von Förderprogrammen leistet die Arbeit damit einen interessanten Beitrag, denn sie gibt Aufschluss in Bezug auf die Zielgruppenspezifität, die institutionelle Ausgestaltung des Programmablaufs und weitere Merkmale, und sie zeigt darüber hinaus, dass Förderprogramme wie andere „entrepreneurial opportunities“ zu behandeln sind.

Der Arbeit ist ein Leserkreis zu wünschen, der weit über die Wissenschaft hinaus bis in die Politik und Verwaltung reichen sollte.

Prof. Dr. Kilian Bizer Georg-August-Universität Göttingen 
Verena Mertins - 978-3-631-75685-0

Downloaded from PubFactory at 01/11/2019 03:00:43AM

via free access 


\section{Danksagung}

Die vorliegende Arbeit entstand im Rahmen meiner Tätigkeit als wissenschaftliche Mitarbeiterin am Lehrstuhl für Wirtschaftspolitik und Mittelstandsforschung an der Georg-August-Universität Göttingen. Dem Inhaber des Lehrstuhls, meinem Doktorvater Herrn Prof. Dr. Kilian Bizer, danke ich für die fachliche Begleitung dieser Arbeit, den großzügig gewährten Forschungsfreiraum und das stete Vertrauen in meine Fähigkeiten. Herrn Prof. Dr. Rolf Sternberg bin ich für die Übernahme des Zweitgutachtens und seine Anregungen zur Empirie zu Dank verpflichtet. Herrn Prof. Dr. Klaus Möller danke ich für seine Bereitschaft, als Drittprüfer meiner Disputation beizuwohnen.

Der empirische Teil dieser Arbeit basiert auf einer Unternehmensbefragung, die ohne die Unterstützung von Herrn Dr. Ole Janssen (Niedersächsisches Ministerium für Wirtschaft, Arbeit und Verkehr), Frau Dr. Anja Altmann (NBank), Frau Dr. Hildegard Sander (Vereinigung der Handwerkskammern Niedersachsen), Herrn Christian Treptow (Industrie- und Handelskammer Hannover) und die Hilfe der Innovationsberater der niedersächsischen Handwerkskammern nicht möglich gewesen wäre. Ein besonderer Dank gilt Herrn Reiner Strunk-Lissowski von der Handwerkskammer Hildesheim-Südniedersachsen und Herrn Dr. Hans-Jürgen Buß vom Innovationszentrum Niedersachsen für ihre Bereitschaft, sich für ein Interview zur Verfügung zu stellen und jederzeit meine Fragen zum Niedersächsischen Innovationsförderprogramm zu beantworten. Für fachliche Anregungen im Bereich Handwerk bedanke ich mich außerdem bei Herrn Dr. Klaus Müller (Volkswirtschaftliches Institut für Mittelstand und Handwerk).

Den im Folgenden aufgeführten Kollegen und Freunden habe ich in besonderer Weise für ihre Unterstützung durch fachliche Anregungen, lebhafte Diskussionen und kritisches Korrekturlesen zu danken: Corinna Ahlfeld, Dr. Nils Bedke, Petra Enß, Ralph Henger, Anja Sölter, Jörg Thomä und Anja Wendt. Für die Hilfe bei der Durchführung der Unternehmensbefragung bedanke ich mich bei den studentischen Hilfskräften des Lehrstuhls.

Mein besonderer Dank gebührt Torsten Elvers für seine unschätzbare Hilfe bei der Endkorrektur und dem Layout dieser Arbeit sowie seine außerordentliche Geduld. Meiner Mutter Gesa Mertins danke ich für ihre große Hilfe beim Korrekturlesen und ihre jederzeitige, unbedingte Unterstützung. Ihr und meinem Großvater Kurt Niemann ist diese Arbeit gewidmet. 
Verena Mertins - 978-3-631-75685-0

Downloaded from PubFactory at 01/11/2019 03:00:43AM

via free access 


\section{Inhaltsverzeichnis}

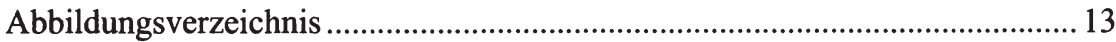

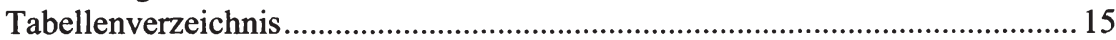

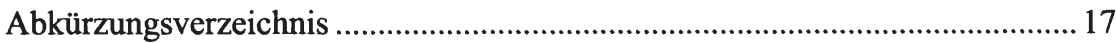

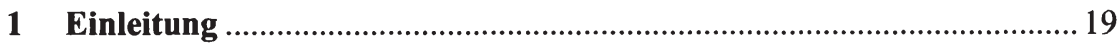

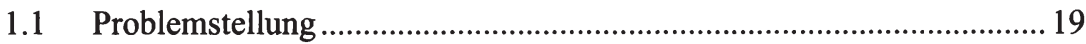

1.2 Stand der Literatur und Forschungsfragen ............................................2 24

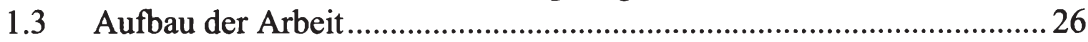

2 Innovationsförderung als Mittel der Wirtschaftspolitik ...................... 29

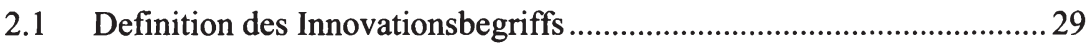

2.2 Ordnungspolitische Einordnung von direkter Innovationsförderung ... 31

2.3 Marktversagen als Begründung für Innovationsförderung ................... 32

2.3.1 Positive externe Effekte von F\&E und Innovation ......................... 32

2.3.2 F\&E und Innovationen als öffentliche Güter ..................................34

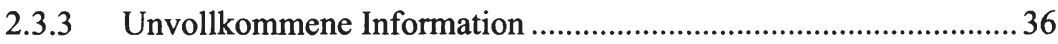

2.3.4 Gefahr einer Überinvestition in die Innovationstätigkeit ................41

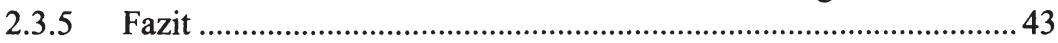

2.4 Wachstumseffekte als Begründung für Innovationsförderung .............43

2.5 Effizienz, Treffsicherheit und Informationsanforderungen ...................47

2.6 Zusammenfassung der Ergebnisse und Hypothesen ..............................51

3 Theorie der Neuen Institutionenökonomik .........................................53

3.1 Grundlegende Theorieansätze der Neuen Institutionenökonomik........53

3.1.1 Unvollständige Information und „bounded rationality“..................55

3.1.2 Der Transaktionskostenansatz .........................................................57

3.1.3 Informationsasymmetrien und Prinzipal-Agent-Beziehungen....... 59

3.2 Politiker in der Neuen Politischen Ökonomie.......................................66

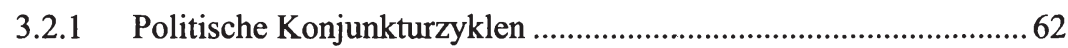

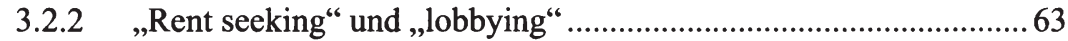

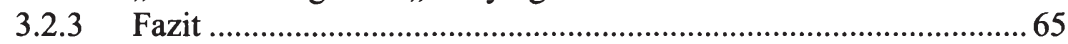

3.3 Bürokraten als Verwalter der Wirtschaftsfördermittel..........................65

3.3.1 Die Bürokratie als Budgetmaximierer nach Niskanen ....................66

3.3.2 Einschränkungen der Macht der Bürokraten ..................................6 68

3.3.3 Alternative Theorieansätze: Risikoaversion und Maximierung des „slack“ 


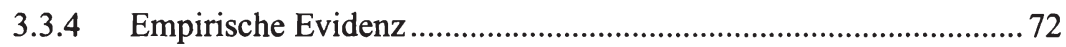

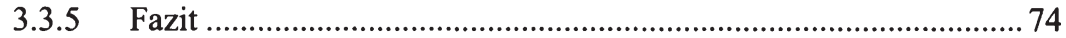

3.4 Zusammenfassung der Ergebnisse ................................................. 75

4 Institutionenökonomische Untersuchung des Niedersächsischen

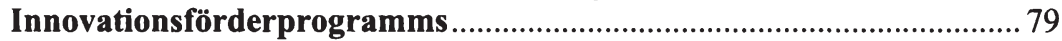

4.1 Der europarechtliche Rahmen von Wirtschaftsförderung.................... 79

4.2 Institutionelle Ausgestaltung des NIFP ..................................................8 82

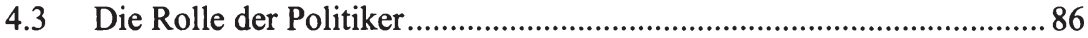

4.3.1 Die Rolle der regionalen Politiker im NIFP ….............................87

4.3.2 Das politische Interesse an Innovationsförderung.......................... 88

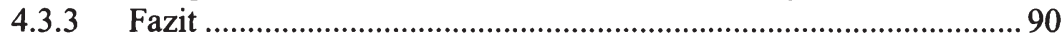

4.4 Die Rolle der Bürokratie ................................................................91

4.4.1 Die Einordnung der Bürokratiegruppen in den Ablauf des NIFP.. 94

4.4.2 Prinzipal-Agent-Beziehung zwischen Politik und Bürokratie ....... 96

4.4.3 Spezifische Interessen der verschiedenen Bürokratengruppen ...... 99

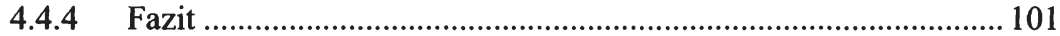

4.5 Die Unternehmer als Fördermittelempfänger des NIFP .................... 102

4.5.1 Prinzipal-Agent-Beziehung zwischen Bürokratie und Unternehmern ............................................................................ 102

4.5.1.1 Erste Informationsasymmetrie: die Auswahl der zu fördernden Projekte .................................................................. 102

4.5.1.2 Zweite Informationsasymmetrie: „rent seeking“ und Mitnahmeeffekte .................................................................. 103

4.5.2 Die Wahrnehmung von Förderangeboten ................................... 107

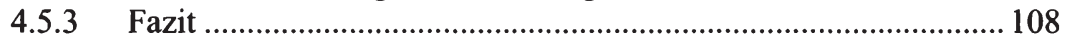

4.6 Zusammenfassung der Ergebnisse und Hypothesen ........................... 109

5 Die Entrepreneurship-Theorie und ihre Anwendung auf Innovationsförderung ........................................................................... 113

5.1 Der Unternehmer in der volkswirtschaftlichen Theorie - die

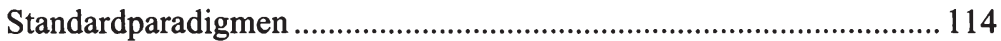

5.2 Grundlegendes zum Unternehmer und zum Entrepreneur.................. 117

5.2.1 Der Begriff des Unternehmers........................................................ 117

5.2.2 Die Unterscheidung zwischen Unternehmer und Entrepreneur... 119

5.3 Unternehmerische Gelegenheiten (,entrepreneurial opportunities“)

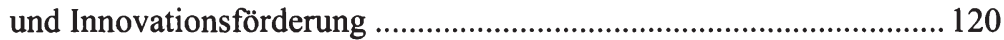

5.4 Erste Phase: Entdeckung der EO ......................................................... 123

5.4.1 Die Reaktion auf die komplexe Umgebung nach von Hayek ...... 124 
5.4.2 Die Aufmerksamkeit des Unternehmers nach von Mises und Kirzner .......................................................................................... 125

5.4.3 Moderne Theorieansätze: die Urteilsfähigkeit nach Casson ........ 128

5.4.4 Empirische Untersuchungen..................................................... 130

5.4.5 Anwendung der Theorie auf Innovation und Innovations-

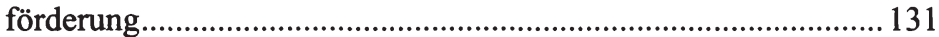

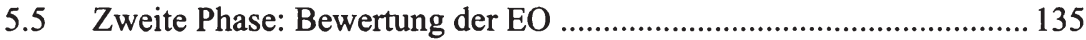

5.5.1 Der Entrepreneur als Risikoträger nach Cantillon ........................ 136

5.5.2 Unterscheidung zwischen Risiko und Unsicherheit nach Knight 137

5.5.3 Moderne Theorieansätze.......................................................... 139

5.5.4 Empirische Untersuchungen..................................................... 139

5.5.5 Anwendung der Theorie auf Innovation und Innovations-

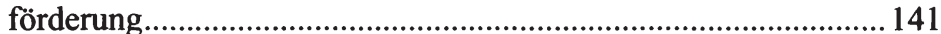

5.6 Dritte Phase: Ausbeutung der EO …….......................................... 144

5.6.1 Vorarbeiten: von Thünen und die Historische Schule................... 144

5.6.2 Die Durchsetzung neuer Kombinationen ..................................... 145

5.6.3 Der Schumpeter'sche Entrepreneur und Wirtschaftswachstum... 146

5.6.4 Anwendung der Theorie auf Innovationsförderung ..................... 148

5.7 Zusammenfassung der Ergebnisse und Hypothesen ............................ 149

6 Empirische Untersuchung des Niedersächsischen Innovations-

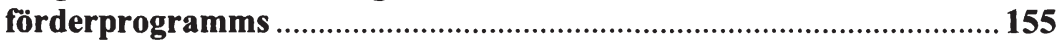

6.1 Auswahl der zu befragenden Unternehmen ....................................... 155

6.2 Verschickung des Fragebogens und Rücklauf .................................. 157

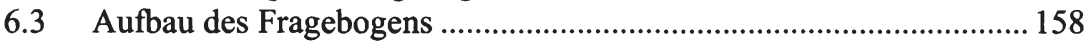

6.4 Vergleich der geförderten und nicht geförderten Unternehmen in

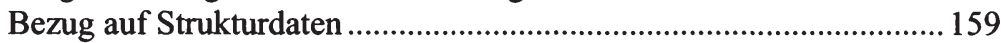

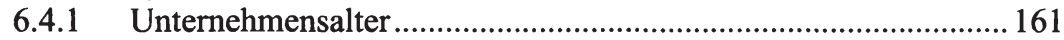

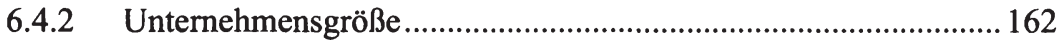

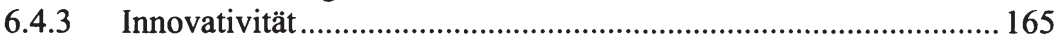

6.4.4 Bewertung von Innovationshemmnissen................................... 172

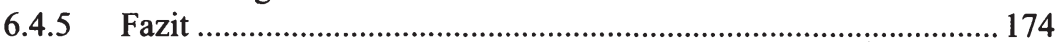

6.5 Vergleich der geförderten und nicht geförderten Unternehmen in Bezug auf Unternehmereigenschaften.............................................. 174

6.5.1 Die Informiertheit des Unternehmers ...................................... 175

6.5.1.1 Informationsverhalten bzgl. Wirtschaftsförderung ................ 179

6.5.1.2 Informiertheit in Bezug auf den Bewerbungsaufwand bei Innovationsförderprogrammen ............................................. 184

6.5.1.3 Informationsverhalten bzgl. Innovation ............................. 187

6.5.1.4 Informiertheit der befragten Handwerksunternehmen .......... 190

6.5.1.5 Zusammenfassung der Ergebnisse.......................................... 193 
6.5.2 Die Risikoeinstellung des Unternehmers ..................................... 193

6.5.3 Bewertung von Unternehmereigenschaften ...............................200

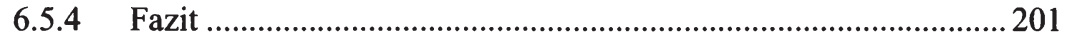

6.6 Logit-Modell (logistische Regression) ............................................... 202

6.7 Zusammenfassung der Ergebnisse und Beantwortung der Hypothesen ...........................................................................................2209

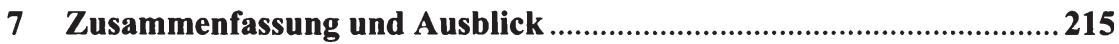

7.1 Zusammenfassung der Ergebnisse ..............................................2215

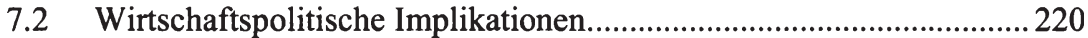

7.2.1 Zielgruppe der Förderung .........................................................220

7.2.2 „Förderdschungel“....................................................................222

7.2.3 Beratungsangebote in der Förderlandschaft ................................2224

7.2.4 Bewerbungsaufwand um Innovationsförderung ...........................225

7.2.5 Institutionelle Ausgestaltung des Programmablaufs ..................... 226

7.2.6 Erfolgskontrolle des Programms ...................................................227

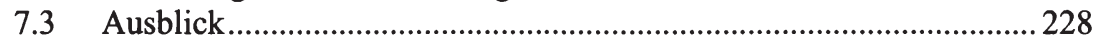

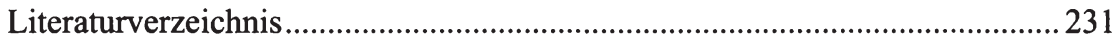

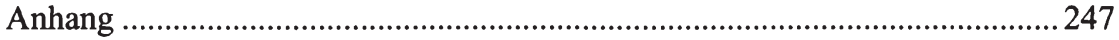




\section{Abbildungsverzeichnis}

Abbildung 1: Bruttoinlandsausgaben für F\&E in Prozent vom BIP für 2006... 20 Abbildung 2: Bruttoinlandsausgaben für F\&E nach finanzierenden Akteuren.. 21 Abbildung 3: Staatliche Ausgaben für F\&E nach durchführenden Akteuren .... 22 Abbildung 4: Allokatives Marktversagen bei Vorliegen eines positiven

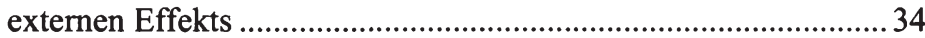

Abbildung 5: Nutzenbewertung von riskanten Investitionsprojekten ..................38 Abbildung 6: Wirkungsweise einer Pigou'schen Subvention ............................. 48 Abbildung 7: Wirkungsweise einer Subvention nach dem Preis-Standard-

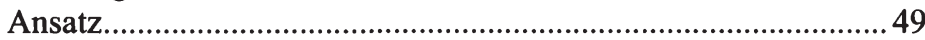

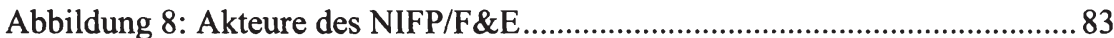

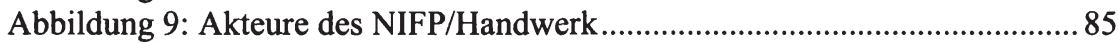

Abbildung 10: Ablauf der Skizzenprüfung .................................................. 95

Abbildung 11: Funktion des Ausfüllenden im Unternehmen ........................... 159

Abbildung 12: Boxplot des Unternehmensalters aufgeteilt nach Förderung.... 162

Abbildung 13: Boxplot der Beschäftigtenverteilung nach Förderung............... 163

Abbildung 14: Säulendiagramm der Beschäftigtenverteilung nach

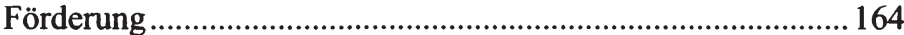

Abbildung 15: Boxplot der Innovationsintensität nach Förderung ................... 169

Abbildung 16: Boxplot des Beschäftigtenanteils im Bereich F\&E nach

Förderung ............................................................................. 170

Abbildung 17: Boxplot der Patente nach Förderung …................................... 170

Abbildung 18: Boxplot des Anteils von Marktneuheiten am Unternehmensumsatz nach Förderung ........................................................... 171

Abbildung 19: Innovationshemmnisse nach Förderung .................................. 172

Abbildung 20: Bewertung der Informationsquellen im Bereich Wirtschaftsförderung

Abbildung 21: Bewertung der Informationsquellen im Bereich Innovation .... 178 Abbildung 22: Uninformiertheit nach Förderung ............................................. 179

Abbildung 23: Uninformiertheit bezogen auf Wirtschaftsförderung ............... 183

Abbildung 24: Zeitaufwand für die Erstellung der Antragskizze...................... 185

Abbildung 25: Zeitaufwand für die Erstellung des Förderantrags ................... 185

Abbildung 26: Zeitaufwand für die Erstellung der Dokumentation der

Fördermittelverwendung......................................................... 186

Abbildung 27: Uninformiertheit bezogen auf den Bereich Innovation ............ 190

Abbildung 28: Bewertung der Informationsquellen im Bereich Wirtschaftsförderung - nur Handwerksunternehmen

Abbildung 29: Bewertung der Informationsquellen im Bereich Innovation nur Handwerksunternehmen ................................................... 192

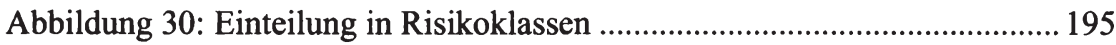


Abbildung 31: Risikoeinstellung nach Förderung 197 Abbildung 32: Risikoeinstellung nach Innovativität 198 


\section{Tabellenverzeichnis}

Tabelle 1: Befragte Unternehmen nach Förderung .......................................... 160

Tabelle 2: Befragte Unternehmen nach Kammerzugehörigkeit ....................... 160

Tabelle 3: Strukturdaten der befragten Unternehmen ....................................... 161

Tabelle 4: Befragte Unternehmen geordnet nach Innovativität ........................ 166

Tabelle 5: Signifikanz der Gruppenunterschiede bezogen auf Innovativitäts-

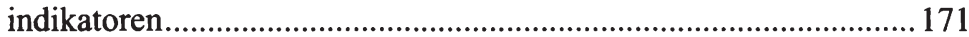

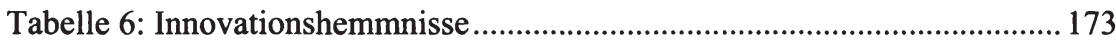

Tabelle 7: Informationsquellen für Wirtschaftsförderung differenziert nach

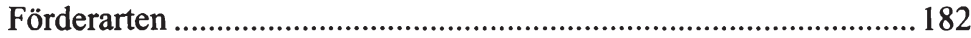

Tabelle 8: Zeitaufwand für die Bewerbung und Dokumentation ...................... 186

Tabelle 9: Ergebnisse des Wilcoxon-Rangsummentest für den Zeitaufwand .. 187

Tabelle 10: Informationsquellen für Innovation differenziert nach

Förderarten nur für innovativ tätige Unternehmen

Tabelle 11: Differenzierte Ergebnisübersicht der Risikoaversion nach

Förderung

Tabelle 12: Differenzierte Ergebnisübersicht der Risikoaversion nach Innovativität in absoluten und relativen Zahlen.............................. 198

Tabelle 13: Ergebnisse des Chi-Quadrat-Unabhängigkeitstests ...................... 199

Tabelle 14: Bewertung der Unternehmereigenschaften.................................... 201

Tabelle 15: Branchen der befragten Unternehmen ...........................................203

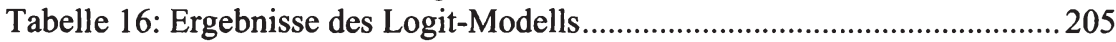

Tabelle 17: Teilnahme an Förderprogrammen.................................................... 223

Tabelle 18: Informationsquellen für Innovation bezogen auf nicht geförderte und durch Innovationsförderung geförderte Unternehmen ...........267

Tabelle 19: Ergebnisse des Logit-Modells nur für innovativ tätige Unternehmen 


\section{Abkürzungsverzeichnis}

$\begin{array}{ll}\text { ABl. } & \text { Amtsblatt } \\ \text { Abs. } & \text { Absatz } \\ \text { Aufl. } & \text { Auflage } \\ \text { Art. } & \text { Artikel } \\ \text { BGBl. } & \text { Bundesgesetzblatt } \\ \text { BIP } & \text { Bruttoinlandsprodukt } \\ \text { BMWi } & \text { Bundesministerium für Wirtschaft und Technologie } \\ \text { bzgl. } & \text { bezüglich } \\ \text { ca. } & \text { circa } \\ \text { d. h. } & \text { das heißt } \\ \text { EFRE } & \text { Europäischer Fonds für regionale Entwicklung } \\ \text { EG } & \text { Europäische Gemeinschaften } \\ \text { EO } & \text { „entrepreneurial opportunity“ } \\ \text { et al. } & \text { et alii (und andere) } \\ \text { etc. } & \text { et cetera } \\ \text { EU } & \text { Europäische Union } \\ \text { F\&E } & \text { Forschung und Entwicklung } \\ \text { f./ff. } & \text { folgende/fortfolgende } \\ \text { Fn. } & \text { Fußnote } \\ \text { FTE } & \text { Forschung und technologische Entwicklung } \\ \text { GA } & \text { Gemeinschaftsaufgabe „Verbesserung der regionalen Wirt- } \\ & \text { schaftsstruktur“ } \\ \text { HwO } & \text { Handwerksordnung } \\ \text { i. O. } & \text { in Ordnung } \\ \text { Kfz } & \text { Kraftfahrzeug } \\ \text { KMU } & \text { kleine und mittlere Unternehmen } \\ \text { Lkw } & \text { Lastkraftwagen } \\ \text { MBl. } & \text { Ministerialblatt } \\ \text { Mio. } & \text { Million } \\ \text { Mrd. } & \text { Milliarde } \\ \text { MW } & \text { Niedersächsisches Ministerium für Wirtschaft, Arbeit und } \\ \text { MU } & \text { Verkehr } \\ \text { NACE } & \text { Niedersächsisches Ministerium für Umwelt und Klimaschutz } \\ & \text { Nomenclature statistique des activités économiques dans la } \\ \text { NBank } & \text { Communauté européenne } \\ \text { Nds. } & \text { Investitions- und Förderbank Niedersachsen } \\ \text { NIFP } & \text { Niedersächsisch } \\ & \text { Niedersächsisches Innovationsförderprogramm } \\ & \end{array}$


NIFP/F\&E Niedersächsisches Innovationsförderprogramm für Forschungs- und Entwicklungsausgaben

NIFP/Handwerk Niedersächsisches Innovationsförderprogramm für das Handwerk

$\mathrm{Nr}$. Nummer

OECD

Organisation for Economic Co-operation and Development

$\mathrm{Rn}$. Randnummer

RWI

Rheinisch-Westfälisches Institut für Wirtschaftsforschung e.V.

S. Seite

u. a. unter anderem

u. ä. vgl. und Ähnliches vergleiche

z. B.

zum Beispiel 


\section{Einleitung}

\subsection{Problemstellung}

„Die zukünftige Wirtschaftsentwicklung Europas hängt von seiner Fähigkeit ab, innovative und forschungsbasierte Sektoren mit hoher Wertschöpfung, die mit den Weltbesten konkurrieren können, zu schaffen und wachsen zu lassen. "1

Diese Einschätzung der Europäischen Kommission stößt in den Wirtschaftswissenschaften und der Politik auf breite Zustimmung. Erstere beschäftigen sich im Rahmen der Endogenen Wachstumstheorie mit der Bedeutung von Innovation und Forschung und Entwicklung (F\&E) für das Wirtschaftswachstum. ${ }^{2}$ In der Politik hingegen gilt das Schlagwort „Innovation“ als Allheilmittel für eine schwächelnde Volkswirtschaft. So werden auf der Ebene der Europäischen Union (EU) Innovation und F\&E als wichtige Bestandteile einer wachsenden Volkswirtschaft angesehen, wie in den Beschlüssen des Europäischen Rats von Lissabon (2000) und Barcelona (2002) hervorgehoben wird. So lautet das Kernziel der von den europäischen Staats- und Regierungschefs verabschiedeten Lissabon-Strategie, unter anderem durch die Förderung von F\&E und Innovationen die EU bis zum Jahr 2010 zum ,wettbewerbsfähigsten und dynamischsten wissensbasierten Wirtschaftsraum in der Welt ${ }^{\text {“3 }}$ zu machen. Eine Konkretisierung der Lissabon-Strategie erfolgte auf dem Gipfel des Europäischen Rats in Barcelona. Demnach sollen die Gesamtausgaben für F\&E und Innovation in der EU bis zum Jahre 2010 auf ein Niveau von $3 \%$ des BIP steigen. Die Erhöhung soll dabei zu zwei Dritteln vom privaten Sektor finanziert werden. ${ }^{4}$

Anhand von Abbildung 1, die die Gesamtausgaben für F\&E in verschiedenen europäischen Staaten für das Jahr 2006 wiedergibt, wird deutlich, dass dieses Ziel bislang nur von Schweden und Finnland erreicht worden ist. Die meisten europäischen Staaten geben deutlich weniger als $3 \%$ ihres BIP für F\&E bzw. Innovation aus. Für Deutschland beträgt der entsprechende Wert 2,53 \%, was zwar über dem EU-Durchschnitt von 1,84\% liegt, jedoch noch weit von den angestrebten $3 \%$ entfernt ist. In absoluten Zahlen gibt Deutschland 58,2 Mrd. Euro für F\&E aus und bleibt damit um ca. 11 Mrd. Euro hinter dem $3 \%$ Ziel zurück.

1 Kommission der Europäischen Gemeinschaften (2005), Teil II, Rn. 24.

2 Vgl. Romer (1990).

3 Vgl. Europäischer Rat (2000), Schlussfolgerung Nr. 5.

4 Vgl. Europäischer Rat (2002), Teil I, Nr. 47. 


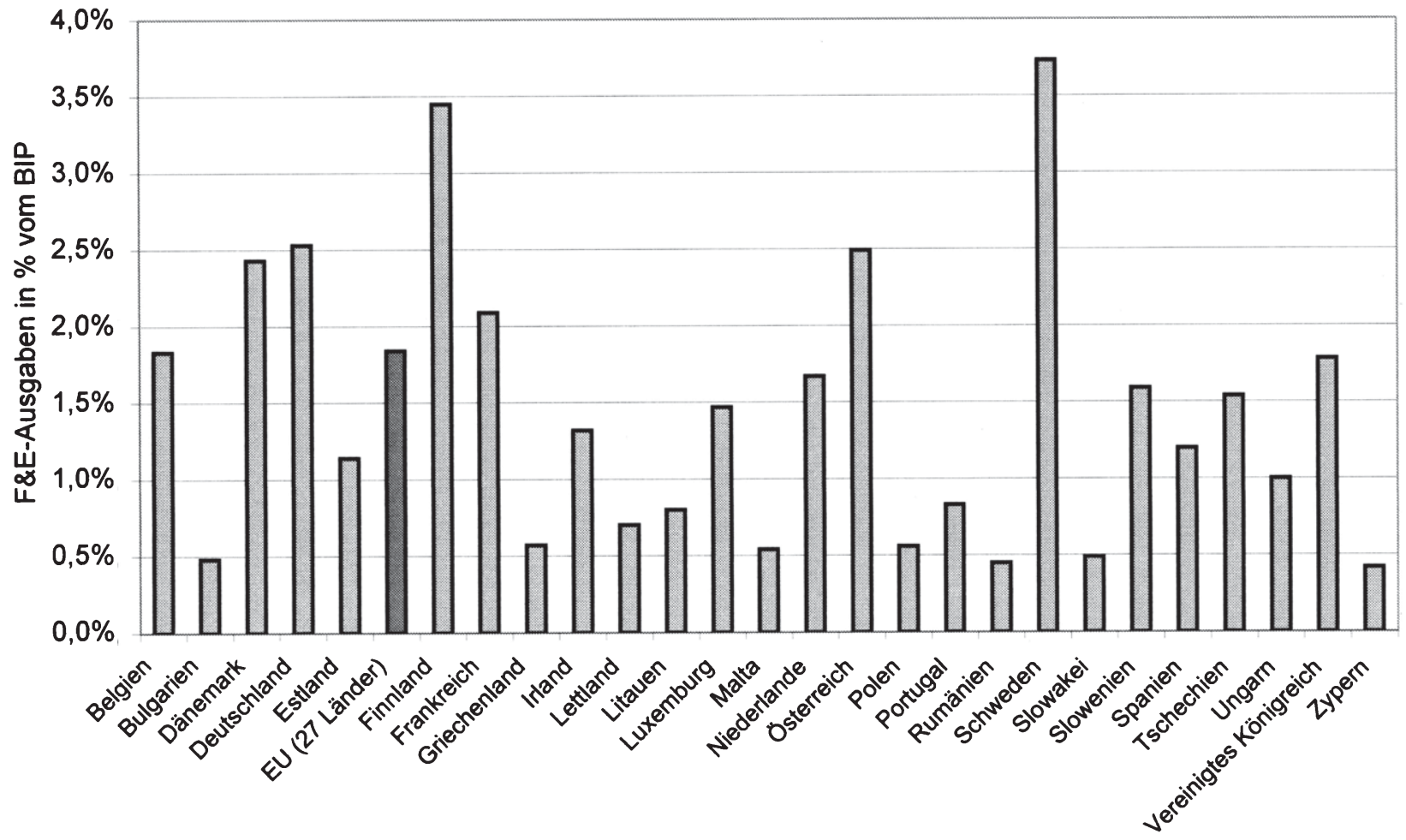

Abbildung 1: Bruttoinlandsausgaben für F\&E in Prozent vom BIP für 2006 (Eigene Darstellung nach Daten von Eurostat. Für Italien lagen noch keine Daten vor. Teilweise sind die Daten vorläufig.). 
Die Gesamtausgaben für F\&E stammen aus verschiedenen Finanzierungsquellen: dem privaten inländischen Sektor, dem öffentlichen inländischen Sektor und dem Ausland. Eine Aufteilung der F\&E-Ausgaben nach finanzierendem Akteur für die Bundesrepublik Deutschland findet sich in Abbildung 2. Die Darstellung zeigt, dass der staatliche Finanzierungsanteil an den gesamten deutschen F\&EAusgaben von 1981 bis 2005 von $41,79 \%$ auf $28,38 \%$ sank. Gleichzeitig stieg der Anteil der Wirtschaft an den gesamten F\&E-Ausgaben von 56,85\% auf $67,57 \%$. Der Finanzierungsanteil des Auslands und der privaten Institutionen ohne Erwerbszweck stellen mit 3,75 bzw. 0,29\% im Jahr 2005 dagegen deutlich geringere Werte dar. Trotz relativ sinkendem Anteil haben sich die staatlichen F\&E-Ausgaben im Zeitraum 1981 bis 2005 mit einer absoluten Erhöhung von 8,12 Mrd. Euro auf 15,82 Mrd. Euro fast verdoppelt und stellen mit einem relativen Anteil von $28,38 \%$ immer noch eine erhebliche Einflussgröße des deutschen F\&E-Verhaltens dar. ${ }^{5}$

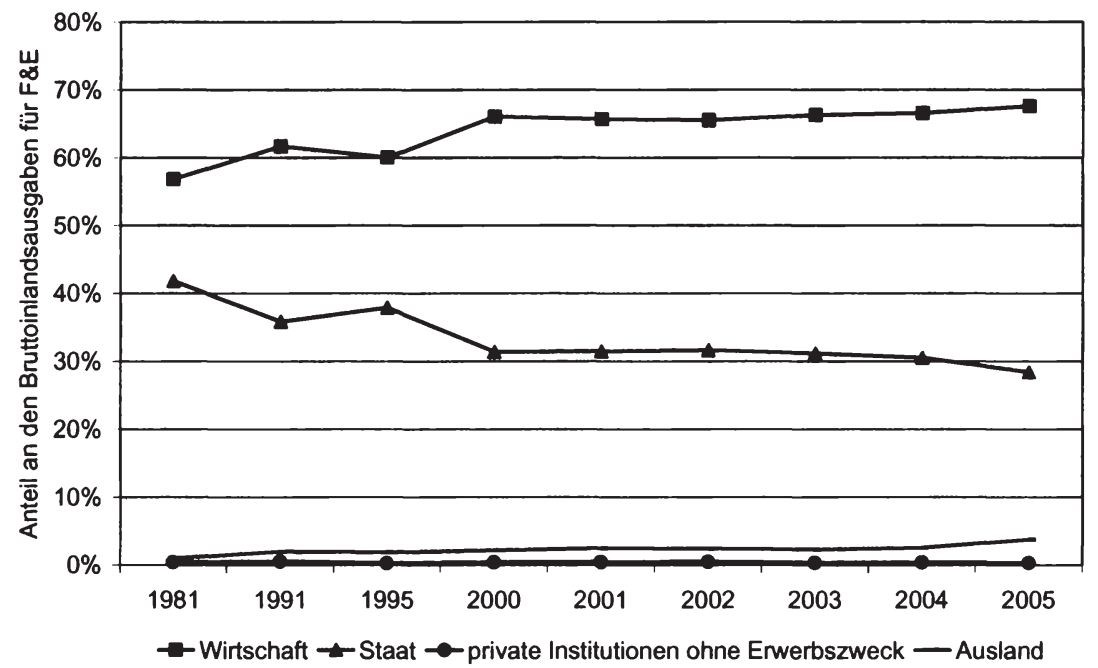

Abbildung 2: Bruttoinlandsausgaben für F\&E nach finanzierenden Akteuren (Eigene Darstellung nach Daten vom Bundesministerium für Bildung und Forschung (2008), S. 492 f.).

Um das $3 \%$-Ziel von Barcelona zu erreichen, kann ein Staat entweder in eigenen Organisationen zusätzliche F\&E bzw. Innovation durchführen lassen oder er kann den privaten Unternehmenssektor mithilfe von indirekten und direkten Fördermaßnahmen zu erhöhten F\&E- bzw. Innovationsinvestitionen anregen. Zu indirekten Fördermaßnahmen gehören beispielsweise Steuererleichterungen für

Vgl. Bundesministerium für Bildung und Forschung (2008), S. $492 \mathrm{f}$. 
F\&E und Wagniskapitalprogramme, während direkte Fördermaßnahmen beispielsweise Auftragsforschung sowie Beihilfen für F\&E oder Innovation im Rahmen einer Projektförderung umfassen. ${ }^{6}$

Bei Betrachtung der Aufteilung der staatlichen Gesamtausgaben für F\&E in Deutschland auf die einzelnen durchführenden Sektoren in Abbildung 3 wird deutlich, dass der staatlich finanzierte Anteil von F\&E der Wirtschaft mit $10,89 \%$ bzw. 1,7 Mrd. Euro im Jahr 2005 keineswegs unerheblich ist. Zwar lag dieser Anteil in der Vergangenheit mit 27,85\% bzw. 2,3 Mrd. Euro im Jahr 1981 sowohl absolut als auch relativ deutlich höher. Jedoch haben sich die staatlich finanzierten Anteile der Hochschulen und der staatlichen Institutionen kontinuierlich erhöht. ${ }^{7}$ Dies spricht zunächst für einen Bedeutungsrückgang der staatlichen Unterstützungsmaßnahmen für F\&E und Innovation im Wirtschaftssektor.

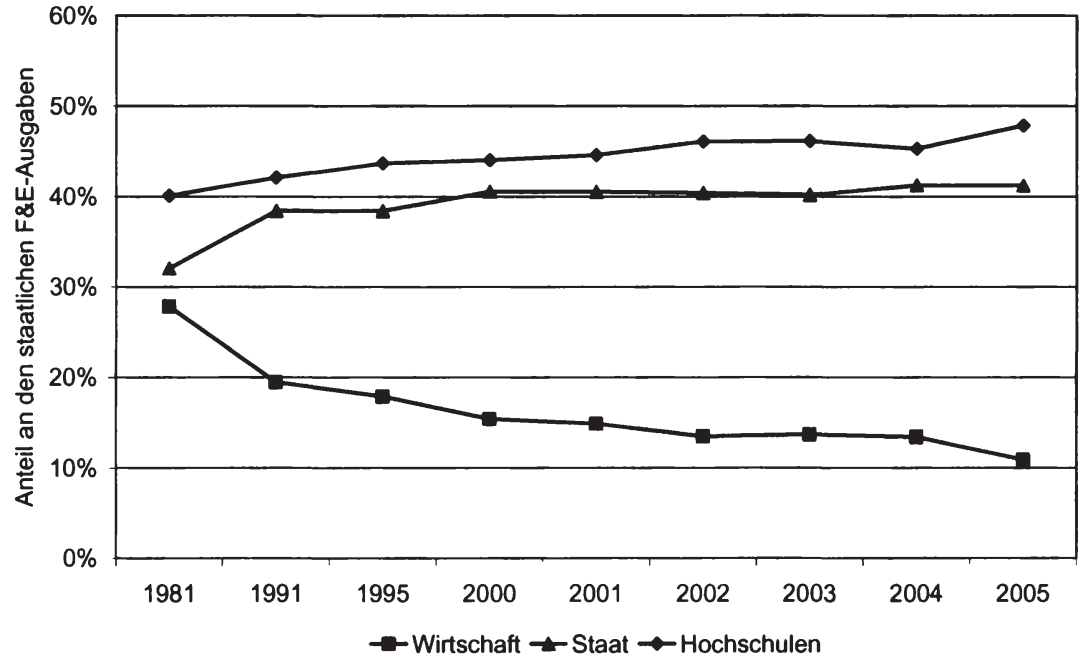

Abbildung 3: Staatliche Ausgaben für F\&E nach durchführenden Akteuren (Eigene Darstellung nach Daten vom Bundesministerium für Bildung und Forschung (2008), S. 492 f.).

Jedoch kommen Rammer und Binz (2006) zu dem Schluss, dass innovative Unternehmen des Verarbeitenden Gewerbes zu einem großen Prozentsatz eine finanzielle Förderung für Innovationsprojekte durch staatliche Stellen in Anspruch nehmen. Am höchsten war der Anteil der unterstützten Unternehmen im Instrumenten- und Fahrzeugbau. So wurden $39 \%$ der innovativen Unternehmen

6 Vgl. Rammer und Binz (2006), S. 134.

7 Vgl. Bundesministerium für Bildung und Forschung (2008), S. 492 f. 
des Instrumentenbaus und $36 \%$ der innovativen Unternehmen des Fahrzeugbaus im Zeitraum 2001-2003 bei ihren Innovationsaktivitäten unterstützt. In den Branchen Chemie/Pharma/Mineralöl, Glas/Keramik/Steinwaren, Maschinenbau, Elektroindustrie und den technischen F\&E-Dienstleistungen lag der Anteil jeweils bei ungefähr $25 \%{ }^{8}$ Diese Zahlen belegen, dass eine staatliche Unterstützung der Innovationsaktivität von einem erheblichen Teil der innovativen Unternehmen in Anspruch genommen wird und in der Praxis ein bedeutsames Phänomen darstellt.

Solche staatliche Unterstützung fällt regelmäßig unter die Beihilfenkontrolle der EU. ${ }^{9}$ Bezogen auf den gesamten Beihilfebereich hat der Europäische Rat in den Schlussfolgerungen von Barcelona betont, dass in der EU die Vergabe von Beihilfen reduziert werden und zielgerichteter erfolgen soll. ${ }^{10}$ Dementsprechend hat die Europäische Kommission im „Aktionsplan staatliche Beihilfen“ festgelegt, dass Innovation und F\&E zu den Schwerpunktbereichen zählen, in denen auch zukünftig Beihilfen vorgesehen sind. ${ }^{11}$ Öffentliche Beihilfen für Innovation und F\&E werden also auch in Zukunft an den privaten Sektor vergeben und beeinflussen den Innovationsprozess im europäischen Wirtschaftsraum.

Die Vergabe von Beihilfen erfolgt auf verschiedenen politischen Ebenen der EU. So stehen deutschen Unternehmen Förderprogramme der EU, des Bundes und der Bundesländer für die Bereiche Innovation und F\&E offen, welche dazu beitragen können, das angesprochene $3 \%$-Ziel in Deutschland zu erreichen. Eines dieser Programme auf Ebene des Landes Niedersachsen ist das Niedersächsische Innovationsförderprogramm (NIFP), welches in dieser Arbeit im Mittelpunkt steht. Dieses Programm wird durch Mittel des Europäischen Fonds für regionale Entwicklung (EFRE) und des niedersächsischen Landeshaushalts finanziert und besteht aus zwei Elementen: erstens dem Niedersächsischen Innovationsförderprogramm für Forschungs- und Entwicklungsausgaben (NIFP/F\&E) und zweitens dem Niedersächsischen Innovationsförderprogramm für das Handwerk (NIFP/Handwerk). Der F\&E-Programmteil ist überaus interessant, da er keinen bestimmten Schwerpunkt der zu fördernden Branchen aufweist und sich in besonderem Maße der Förderung von kleinen und mittleren Unternehmen (KMU) annimmt, die aufgrund ihrer Größe bei der Innovationstätigkeit speziellen Schwierigkeiten unterliegen. Die Ausrichtung auf die Zielgruppe des Handwerks im Rahmen des zweiten Programmteils verdient ebenfalls besondere Beachtung, da speziell die Innovationstätigkeit des Handwerks in der Literatur häufig unterschätzt wird. ${ }^{12}$ Darüber hinaus ist die institutionelle

8 Vgl. Rammer und Binz (2006), S. 140.

9 Vgl. hierzu Abschnitt 4.1.

10 Vgl. Europäischer Rat (2002), Teil III, Wirtschaft und Finanzen, Schlussfolgerung Nr. 16.

11 Vgl. Kommission der Europäischen Gemeinschaften (2005), Teil II, Rn. 25.

12 Vgl. Lahner (2004), S. $1 \mathrm{ff}$. 
Ausgestaltung des NIFP/Handwerk aufgrund der Einbindung von Innovationsberatern der Handwerkskammern in den Entscheidungsprozess über die inhaltliche Bewertung der Projektanträge deutschlandweit einmalig. Eine eingehende Untersuchung dieses Förderprogramms erscheint daher viel versprechend.

\subsection{Stand der Literatur und Forschungsfragen}

Wegen des staatlichen Eingriffs in den Marktmechanismus wird dem Phänomen der Wirtschaftsförderung in der neoklassischen Volkswirtschaftslehre mit besonderer Skepsis begegnet. ${ }^{13}$ Die bisherige Behandlung von Innovationsförderung in der volkswirtschaftlichen Literatur beschränkt sich daher im Wesentlichen auf zwei Bereiche: erstens die Analyse der theoretischen Begründbarkeit von Innovationsförderung und zweitens die Untersuchung der durch die Förderung generierten Effekte.

Der erste Bereich der theoretischen Auseinandersetzung mit dem Phänomen der Innovationsförderung findet im Rahmen von ordnungsökonomischen Analysen statt, die wirtschaftspolitische Rechtfertigungsmöglichkeiten der Förderung von F\&E bzw. Innovation als Eingriff in den Marktmechanismus untersuchen. ${ }^{14}$ Dabei können zum einen das Vorliegen eines allokativen Marktversagens im Bereich F\&E/Innovation durch externe Effekte oder unvollkommene Information als ordnungsökonomische Begründung des Markteingriffs herangezogen werden. Zum anderen bietet der positive Zusammenhang zwischen technischem Fortschritt und Wirtschaftswachstum nach den Erkenntnissen der endogenen Wachstumstheorie eine mögliche Rechtfertigung für staatliche Fördermaßnahmen. ${ }^{15}$

Die wirtschaftspolitische Begründung von Innovationsförderung stellt jedoch nur einen Teilaspekt dieser Arbeit dar. Die Existenz von Innovationsförderung in der EU ist ein wirtschaftspolitisches Faktum, dessen Wirkungen in dieser Arbeit einer näheren Analyse unterzogen werden. Es wird dabei jedoch nicht um die Messung der Effekte der Förderung im Sinne des direkten Einflusses auf volkswirtschaftliche Schlüsselgrößen wie technischer Fortschritt, Wirtschaftswachstum und Beschäftigung gehen. Hierzu liegt bereits eine große Bandbreite an Studien vor, welche die durch die jeweiligen Förderprogramme erzeugten wirtschaftlichen Folgeeffekte abbilden. ${ }^{16}$ Diese Untersuchungen machen den zweiten großen Bereich der volkswirtschaftlichen Literatur zum Thema Innova-

13 Vgl. z. B. Oberender (1987), S. $132 \mathrm{ff}$.

14 Vgl. für den Bereich F\&E/Innovation z. B. Issing (1979), Oberender (1987) und Zeppernick (1985).

15 Vgl. z. B. Romer (1990), S. 97; Klodt (1995), S. 98; Zagler (2007), S. 821 ff.

16 Vgl. für den Bereich F\&E/Innovation z. B. David et al. (2000), Wallsten (2000) und Czarnitzki und Fier (2002). 
tionsförderung aus, der eine empirische Auseinandersetzung mit diesem Phänomen bietet. Die meisten Studien messen den Erfolg von Wirtschaftsförderprogrammen, indem sie die Entwicklung einer Gruppe der durch ein Programm geförderten Unternehmen der Entwicklung einer Kontrollgruppe nicht geförderter Unternehmen gegenüberstellen. Das grundsätzliche Problem dieser Untersuchungen besteht jedoch darin, dass die Zuordnung von Unternehmen zu den beiden Gruppen nicht zufällig erfolgt. ${ }^{17}$ Bestimmte Charakteristika beeinflussen sowohl die Entscheidung des Unternehmens, sich überhaupt um Fördermittel zu bewerben, sowie die positive Bewertung der Förderanträge durch die Fördermittel vergebende Institution als auch die weitere Unternehmensentwicklung. Wenn nun die Entwicklung der Gruppe der geförderten Unternehmen mit der Entwicklung der Kontrollgruppe verglichen wird, dann ist es schwierig den Einfluss der Förderung vom Einfluss dieser unternehmenseigenen Charakteristika zu trennen.

Diese Arbeit hat die Zielsetzung, am Beispiel der Innovationsförderung auf die Spur dieser beschriebenen Charakteristika zu kommen und nimmt zu diesem Zweck eine theoretische und empirische Analyse vor. Es wird daher die Fragestellung behandelt, inwiefern sich Unternehmen, die sich für Innovationsförderprogramme beworben bzw. Innovationsfördermittel erhalten haben, von Unternehmen, die sich nicht für Fördermittel bewarben und dementsprechend auch keine Förderung erhielten, unterscheiden. Zu diesem Zweck wird das in der Volkswirtschaftslehre vorherrschende Modell des Homo Oeconomicus verlassen und die Theorien der Neuen Institutionenökonomik angewendet. Es gibt bislang nur wenige institutionenökonomische Untersuchungen von Wirtschaftsförderung, wie beispielsweise die politökonomische Analyse von Subventionen als Instrument der Wirtschaftspolitik von Hartig (1990) und die institutionenökonomische Untersuchung von Gründungsförderung von Gläser (2002). Diese Arbeit baut auf diesen Studien auf und untersucht die Ausgestaltung von Innovationsförderung am Beispiel des NIFP und die Wirkung von Innovationsförderung auf die beteiligten Akteure unter Berücksichtigung ihrer Anreize, Interessen und Verhaltensweisen. Zum näheren Verständnis der wichtigen Akteursgruppe der Unternehmer, welche vor der Entscheidung stehen, sich für Förderprogramme zu bewerben, werden Theorieansätze aus der Entrepreneurship-Forschung angewandt. Diese Theorieansätze beschäftigen sich im Wesentlichen mit der Fragestellung, inwiefern sich Unternehmer in ihren Eigenschaften und Fähigkeiten von anderen Wirtschaftssubjekten unterscheiden. In dieser Arbeit werden diese Theorien zur Differenzierung innerhalb der Gruppe der Unternehmer verwendet, um auf die Spur einiger der oben angesprochenen Charakteristika zu kommen, die geförderte Unternehmer von nicht geförderten Unternehmern unterscheiden. Die vorgenommene Verknüpfung von Neuer Institutionenökonomik und Entrepreneurship-Theorie trägt in hohem Maße zur Erweiterung des ökonomi-

17 Vgl. Wallsten (2000), S. 88; Görg und Strobl (2007), S. 220 f.; Busom (2000), S. 114. 
schen Verständnisses von Innovationsförderung bei, da hierdurch die Akteursgruppe der Unternehmer detailliert betrachtet und angemessen berücksichtigt werden kann.

Neben den theoretischen Erkenntnissen trägt diese Arbeit mit ihrer Analyse des niedersächsischen Programms als einem besonders interessanten Innovationsförderprogramm zur wirtschaftspolitischen Forschung bei. Bislang existiert nur eine Untersuchung des Handwerksprogrammteils des Vorgängerprogramms von Lahner (2004), die eine innovationstheoretische und handwerksspezifische Betrachtung liefert. Die institutionenökonomische Analyse unter Einbeziehung der Entrepreneurship-Ansätze liefert nicht nur theoretisch hergeleitete Hypothesen, sondern überprüft diese anschließend empirisch anhand einer Befragung von niedersächsischen Unternehmen.

Die in dieser Arbeit gewonnenen Erkenntnisse sind für die Wirtschaftspolitik insbesondere deshalb von praktischer Bedeutung, da sie eine Erklärung dafür liefern, welche Unternehmen sich überhaupt für Innovationsförderprogramme bewerben und in der Folge auch Fördermittel erhalten. Obwohl bei der Beurteilung der Förderwürdigkeit eines Unternehmens das konkrete Innovationsprojekt im Vordergrund steht, können systematische Unterschiede zwischen geförderten und nicht geförderten Unternehmen darauf hindeuten, dass Innovationsförderprogramme nur von einer bestimmten Gruppe von Unternehmern wahrgenommen und für interessant befunden werden, so dass sich letztlich eine bestimmte Klientel überhaupt um diese Fördermittel bewirbt. In diesem Fall schließt sich automatisch die Frage an, ob es sich bei dieser Gruppe um die ursprünglich formulierte Zielgruppe der Förderung handelt oder ob die Ausgestaltung und Vermittlung von Innovationsförderprogrammen womöglich angepasst werden sollte.

\subsection{Aufbau der Arbeit}

Die Arbeit gliedert sich in sieben Kapitel. Im Anschluss an diese Einleitung untersucht Kapitel 2 die Innovationsförderung als Mittel der Wirtschaftspolitik im Rahmen einer ordnungsökonomischen Analyse. Zunächst werden die definitorischen Grundlagen dieser Arbeit gelegt, indem der Innovationsbegriff definiert und vom enger zu verstehenden Begriff der F\&E abgegrenzt wird. Es folgt eine ordnungsökonomische Einordnung von Innovationsförderung als staatlichem Eingriff in den Marktmechanismus unter Berücksichtigung der damit verbundenen möglichen negativen Effekte. Trotzdem kann der Einsatz des wirtschaftspolitischen Instruments der Innovationsförderung ordnungsökonomisch durch Vorliegen eines allokativen Marktversagens oder wachstumstheoretisch durch die mit Innovation verbundenen positiven Wachstumseffekte gerechtfertigt werden. 
Es wird untersucht, welcher Argumentation das in dieser Arbeit als Beispiel herangezogene NIFP folgt.

Nach Prüfung der grundlegenden Rechtfertigung von Innovationsförderung wird das konkrete niedersächsische Beispiel einer institutionenökonomischen Analyse unterzogen. $\mathrm{Zu}$ diesem Zweck werden in Kapitel 3 die grundlegenden Theoriegebäude der Neuen Institutionenökonomik (unvollständige Information, „bounded rationality“, Transaktionskosten, Informationsasymmetrie und Prinzipal-Agent-Beziehung) sowie deren spezielle Theorieansätze der Neuen Politischen Ökonomie und der Theorie der Bürokratie dargestellt, welche sich mit den Akteursgruppen der Politiker und der Bürokraten befassen.

Kapitel 4 widmet sich der eigentlichen institutionenökonomischen Analyse des NIFP. Zunächst wird die konkrete Ausgestaltung des Programms beschrieben. Daran anschließend werden die in Kapitel 3 vorgestellten Theorien konkret auf die am NIFP beteiligten Akteursgruppen angewendet, um ihr jeweiliges Verhalten, ihre Interessen, Anreize und Beziehungen zu erklären. Die verschiedenen Ebenen des Fördersystems mit den einzelnen Gruppen der daran beteiligten Akteure werden differenziert untersucht. Es wird dargelegt, welche Interessen die regionalen Politiker mit dem Beschluss zur Einrichtung eines derartigen Förderprogramms verfolgen. Die Auswahl der zu fördernden Unternehmen durch Bürokraten, die die Fördermittel vergeben, wird ebenfalls einer genauen theoretischen Untersuchung unterzogen.

Jedoch können nur Unternehmen gefördert werden, die sich überhaupt für das Förderprogramm beworben haben. Da die dargestellten Theorieansätze der Neuen Institutionenökonomik für die Beantwortung dieser Frage keine geeigneten Ansatzpunkte aufweisen, wird ein weiterer theoretischer Ansatz herangezogen und in die institutionenökonomische Analyse integriert: die Entrepreneurship-Theorie, welche in Kapitel 5 in ihren für diese Arbeit wesentlichen Konzepten und Zusammenhängen dargestellt wird. Es wird dargelegt, dass sowohl Innovation als auch Innovationsförderung im Sinne von profitversprechenden Möglichkeiten für das Unternehmen als so genannte „entrepreneurial opportunities“ bezeichnet werden können. Die auf das Konzept der „entrepreneurial opportunities" anwendbaren Ansätze der Entrepreneurship-Theorie beschäftigen sich mit Urteilsfähigkeit, Risikoeinstellung und Innovativität von Unternehmern, welche sich in diesen Eigenschaften und Fähigkeiten von anderen Wirtschaftssubjekten unterscheiden. Diese Theorieansätze werden diskutiert und anschließend direkt auf das konkrete Beispiel des NIFP angewendet, indem innerhalb der Gruppe der Unternehmer nach diesen Eigenschaften differenziert wird. Mit Hilfe dieser Theorieansätze können Charakteristika von Unternehmen und Unternehmern bestimmt werden, die maßgebliche Bestimmungsgründe für eine Bewerbung um Fördermittel und die anschließende Förderung darstellen.

Kapitel 6 bildet den empirischen Teil der Arbeit, in dem die Ergebnisse einer im Mai/Juni 2007 im Rahmen dieser Arbeit durchgeführten schriftlichen Un- 
ternehmensbefragung in Niedersachsen dargelegt werden. $\mathrm{Zu}$ den Themen Innovativität und Innovationsförderung wurden Unternehmen befragt, die durch das NIFP Fördermittel erhielten, sowie Unternehmen aus denselben Wirtschaftszweigen, die entweder nicht gefördert wurden oder sich nicht für ein solches Förderprogramm beworben haben. Die in den vorhergehenden Kapiteln aufgestellten Hypothesen werden in diesem Kapitel empirisch überprüft.

Kapitel 7 gibt abschließend einen Überblick über die zentralen Ergebnisse der Arbeit und stellt die aus diesen Ergebnissen resultierenden wirtschaftspolitischen Implikationen dar. 


\section{Innovationsförderung als Mittel der Wirtschaftspolitik}

Dieses Kapitel beschäftigt sich mit der ordnungsökonomischen Einordnung von Innovationsförderung und der Diskussion, unter welchen Umständen Innovationsförderung als Eingriff in den Marktmechanismus zu rechtfertigen ist. Zu diesem Zweck werden nach einer Definition des Innovationsbegriffs (Abschnitt 2.1) und einer ordnungspolitischen Einordnung von Innovationsförderung in der Marktwirtschaft (Abschnitt 2.2) die verschiedenen theoretischen Begründungen für einen staatlichen Eingriff - allokatives Marktversagen (Abschnitt 2.3) und Wachstumseffekte (Abschnitt 2.4) - ausführlich diskutiert. In diesem Zusammenhang wird genauer auf die Frage eingegangen, welche Arten von Unternehmen und Unternehmern vom jeweiligen theoretischen Standpunkt aus gefördert werden sollten. Schließlich wird das Instrument der direkten Innovationsförderung in Hinblick auf Effizienz, Treffsicherheit und Informationsanforderungen für die beteiligten Akteure bewertet (Abschnitt 2.5), bevor ein Fazit und eine abschließende Diskussion erfolgt (Abschnitt 2.6).

\subsection{Definition des Innovationsbegriffs}

Fast alle Definitionen des Innovationsbegriffs gehen auf Schumpeter (1926) zurück, der anstelle des Begriffs Innovation den Ausdruck „Durchsetzung neuer Kombinationen "18 verwendet. Mit dieser Beschreibung betont er zwei Aspekte von Innovationen: die qualitative Neuartigkeit eines Produktes bzw. Verfahrens (Invention) sowie die Durchsetzung am Markt oder innerhalb einer Organisation. Die grundlegenden Innovationen können fünf verschiedene Formen annehmen: „1. Herstellung eines neuen, d. h. dem Konsumentenkreise noch nicht vertrauten Gutes oder einer neuen Qualität eines Gutes. 2. Einführung einer neuen, d. h. dem betreffenden Industriezweig noch nicht praktisch bekannten Produktionsmethode, die keineswegs auf einer wissenschaftlich neuen Entdeckung zu beruhen braucht und auch in einer neuartigen Weise bestehen kann mit einer Ware kommerziell zu verfahren. 3. Erschließung eines neuen Absatzmarktes [...]. 4. Eroberung einer neuen Bezugsquelle von Rohstoffen oder Halbfabrikaten [...]. 5. Durchführung einer Neuorganisation, wie Schaffung einer Monopolstellung [...] oder Durchbrechen eines Monopols "19.

Die beiden grundlegenden von Schumpeter beschriebenen Aspekte einer Innovation, die qualitative Neuartigkeit und die Durchsetzung am Markt, finden

18 Schumpeter (1926), S. 100. Für eine Diskussion weiterer Definitionsmöglichkeiten und Abgrenzungen des Innovationsbegriffes vgl. Hauschildt (1997), S. 1 ff.

19 Schumpeter (1926), S. $100 \mathrm{f}$. 
sich auch im Oslo Manual, den Richtlinien der OECD für Sammlung und Interpretation von Innovationsdaten: "An innovation is the implementation of a new or significantly improved product (good or service), or process, a new marketing method, or a new organisational method in business practices, workplace organisation or external relations. "20 Innovationsaktivitäten werden dementsprechend definiert als: ,all those scientific, technological, organisational, $f i$ nancial and commercial steps, including investment in new knowledge, which actually lead to, or are intended to lead to, the implementation of innovations " 21

Der Innovationsbegriff, der in der in Abschnitt 4.2 näher erläuterten „Richtlinie über die Gewährung von Zuwendungen im Rahmen des Niedersächsischen Innovationsförderprogramms “22 (im Folgenden NIFP-Richtlinie) verwendet wird, enthält ebenfalls die wesentlichen beiden Aspekte der Schumpeter'schen Definition. So müssen die geförderten Vorhaben gemäß Ziffer 4.2 der NIFP-Richtlinie ,eine Neuheit in der Bundesrepublik Deutschland darstellen. Ein Vorhaben gilt als neu, wenn sich die zu entwickelnden Produkte, Verfahren oder Dienstleistungen noch nicht auf dem Markt befinden und nicht wirtschaftlich verwertet wurden. Die Weiterentwicklung von Technologien und Techniken für bereits auf dem Markt befindliche Produkte, Verfahren oder Dienstleistungen kann gefördert werden, wenn die Weiterentwicklung zur erheblichen Verbesserung oder wesentlichen Änderung des bisherigen Entwicklungsergebnisses führt. " Hier wird das Kriterium der qualitativen Neuheit von Schumpeter deutlich. Das Kriterium der Durchsetzung am Markt findet sich in Ziffer 4.4 der Richtlinie: „Vorhaben sollen mittelfristig die Aussicht auf eine wirtschaftliche Verwertbarkeit bzw. einen wirtschaftlichen Erfolg erkennen lassen. "

Um ein neuartiges Produkt oder Produktionsverfahren zu erzeugen, muss F\&E durchgeführt werden. Die Bereiche F\&E und Innovation gehören deswegen untrennbar zusammen ${ }^{23}$, so dass bei der ordnungsökonomischen Einordnung von Innovationsförderung auf die Erkenntnisse aus der F\&E-Forschung zurückgegriffen werden kann.

20 Vgl. OECD und Eurostat (2005), S. 46 f.

21 Vgl. OECD und Eurostat (2005), S. 91.

22 Gemeinsamer Runderlass des Niedersächsischen Ministeriums für Wirtschaft, Arbeit und Verkehr und des Ministeriums für Umwelt vom 28.04.2004 (Nds. MBI. Nr. 19/2004, S. 415).

23 Für eine Darstellung des Innovationsprozesses aus betriebswirtschaftlicher Sicht vgl. Brockhoff (1999), S. 38 f. 


\subsection{Ordnungspolitische Einordnung von direkter Innovations- förderung}

Die wesentlichen konstituierenden Merkmale einer Marktwirtschaft sind neben dem Privateigentum an Produktionsmitteln die dezentralen Entscheidungen der Wirtschaftsakteure über die von ihnen angebotenen und nachgefragten Güter. Die Koordination von Angebot und Nachfrage erfolgt auf dem Markt über den Wettbewerb und den sich bildenden Marktpreis. Leistungsanreize sind gegeben durch die Möglichkeit der Gewinnerzielung für den Unternehmer und den Erwerb von Lohneinkommen für die Arbeitnehmer. ${ }^{24}$

Es handelt sich bei Innovationsförderung um eine spezielle Form von Subventionen, also Geldzahlungen oder geldwerte Leistungen der öffentlichen Hand an Unternehmen, in deren Gegenzug die öffentliche Hand bestimmte Verhaltensweisen erwartet bzw. sich davon verspricht. ${ }^{25}$ Innovationsförderung greift in den Marktmechanismus auf direkte und indirekte Weise ein. Die direkte Form von Innovationsförderung besteht beispielsweise aus Zuschüssen und vergünstigten Darlehen für bestimmte Innovationsprojekte und einzelne Unternehmen, während Steuererleichterungen und generelle Investitionszulagen Mittel der indirekten Innovationsförderung für alle innovativ tätigen Unternehmen darstellen. $\mathrm{Da}$ es in dieser Arbeit vorrangig um das NIFP als ein Beispiel für direkte Innovationsförderung in Form von Zuschüssen geht, beschränkt sich die vorliegende Arbeit auf die Bewertung dieser speziellen Förderform. ${ }^{26}$

Bei der Untersuchung von direkter Innovationsförderung als eine Form von Subvention auf ihre generelle Systemkonformität mit den marktwirtschaftlichen Prinzipien wird der erhebliche staatliche Eingriff in den Marktmechanismus deutlich. Die freie Bildung des Marktpreises und somit der Allokationsmechanismus des Marktes werden durch die Verwendung von Subventionen insofern beeinträchtigt, als dass subventionierte Unternehmen ihre Güter zu niedrigeren Preisen produzieren und anbieten können als ihre nicht subventionierten Wettbewerber. In der Folge kann der Marktpreis sinken und eine Gewinnverschiebung zwischen geförderten und nicht geförderten Unternehmen stattfinden. Die Gewinnsituation der subventionierten Unternehmen verbessert sich, so dass es zu einer Verschlechterung der Wettbewerbssituation der nicht subventionierten Unternehmen kommt. Es liegt eine Sozialisierung der Verluste vor, da alle Unternehmen und Bürger über ihre Steuerzahlungen die Subventionen mit finanzieren, so dass die Möglichkeit besteht, dass subventionierte Unternehmen eigentlich wettbewerbsfähige Unternehmen vom Markt verdrängen und der Wettbe-

24 Vgl. Gutmann (1988), S. 140 ff.; Fritsch et al. (2003), S. 6 ff.

25 Vgl. Hansmeyer (1977), S. 960.

26 Zur ökonomischen Bewertung von direkter und indirekter Innovationsförderung im Vergleich vgl. Issing (1979), S. 44 ff.; Oberender (1987); Klodt (1995), S. 114 f. 
werbsmechanismus verfälscht wird. ${ }^{27}$ Aus diesem Grund besteht in der EU auch ein generelles Beihilfenverbot (vgl. Abschnitt 4.1).

Weitere Kritikpunkte an Subventionen sind neben mittel- und langfristig auftretenden Beharrungs- und Kumulationstendenzen die Zerstörung der unternehmerischen Risikobereitschaft, da Unternehmer die Durchführung von Investitionen von der Subventionsvergabe abhängig machen könnten und ohne Subventionen keine Investitionstätigkeit mehr vornehmen. ${ }^{28}$

Um Innovationsförderung trotz der genannten allgemeinen Subventionskritik als wirtschaftspolitischen Eingriff theoretisch zu rechtfertigen, gibt es zwei mögliche Argumentationen. Zum einen kann die Innovationstätigkeit mit einem allokativen Marktversagen verbunden sein (vgl. folgender Abschnitt), zum anderen können Innovationen positive Wachstumseffekte für die Volkswirtschaft erzeugen, worauf in Abschnitt 2.4 eingegangen wird. Im Folgenden wird näher erläutert, dass nicht alle der oben genannten Argumente der allgemeinen Subventionskritik auch für den Spezialfall der Innovationsförderung gelten. Denn die künstliche Veränderung der Güterpreise, die Wettbewerbsverzerrung zugunsten der geförderten innovativen Unternehmen und die Übernahme eines Teiles des unternehmerischen Risikos durch die Subvention entsprechen genau der mit Innovationsförderung verbundenen Zielsetzung, wie im folgenden Abschnitt gezeigt wird.

\subsection{Marktversagen als Begründung für Innovationsförderung}

Um Wirtschaftsförderung aus ökonomischer Sicht mit dem Vorliegen eines allokativen Marktversagens zu rechtfertigen, müssen entweder externe Effekte oder unvollkommene Information vorliegen, unabhängig davon, um welche Form der Wirtschaftsförderung es sich im Einzelfall handelt. ${ }^{29}$ Die Europäische Kommission gibt an, dass der Innovationsprozess durch Marktversagen gestört werden kann, so dass öffentliche Beihilfen in diesem Bereich gerechtfertigt seien. ${ }^{30}$ Inwiefern diese Argumentation zutrifft, wird im Folgenden untersucht.

\subsubsection{Positive externe Effekte von $F \& E$ und Innovation}

Die erste Begründung für Marktversagen liegt vor, wenn Unternehmen nicht ausschließen können, dass Teile der Volkswirtschaft, wie z. B. Konkurrenten,

27 Vgl. Harzem (1988), S. 39 f.; Röpke (1929), S. 877; Bundesministerium der Finanzen (2006), S. 8; Oberender (1987), S. 132 ff.

28 Vgl. Hartig (1990), S. 79 f.; Berthold (1967), S. 117 f.; Oberender (1987), S. 134 f.; Zeppernick (1985), S. 79; Casson (2001), S. 532.

29 Vgl. Hansmeyer (1977), S. 980; Issing (1979), S. 34 f.

30 Vgl. Kommission der Europäischen Gemeinschaften (2005), Teil II, Rn. 25. 
von ihrer F\&E-Tätigkeit profitieren, welche die Basis für die Entwicklung innovativer Produkte bildet (externer Grenzertrag). Die privaten Grenzerträge der Unternehmen durch die F\&E-Tätigkeit (bestimmt durch die Nachfrage der Konsumenten nach den innovativen Produkten) weichen von den sozialen Grenzerträgen (private Grenzerträge addiert um den externen Grenzertrag durch die Innovationstätigkeit) ab. Da die Unternehmen nun bei Festlegung des Ausmaßes ihrer F\&E-Tätigkeit nur ihre privaten Grenzerträge berücksichtigen und ihren privaten Grenzkosten der F\&E-Produktion gleichsetzen, ist ihre F\&E-Tätigkeit geringer als die gesamtgesellschaftlich optimale Menge. ${ }^{31}$

Der Zusammenhang kann graphisch in einem Preis-Mengen-Diagramm abgebildet werden. Die privaten Grenzkosten (unternehmerische Angebotskurve) steigen mit zunehmender Produktionsmenge. Die sozialen Grenzerträge ergeben sich aus der Summe der privaten Grenzerträge (private Nachfrage) und dem zusätzlichen externen Grenznutzen. Wie Abbildung 4 zeigt, ergibt sich als Marktgleichgewicht der Punkt $C$ im Schnittpunkt von Angebot und Nachfrage mit einer Menge in Höhe von X' und einem Preis in Höhe von P'. Das gesamtwirtschaftliche Optimum liegt allerdings im Punkt D, wo sich Angebotskurve und soziale Grenzerträge schneiden, mit einer höheren Menge in Höhe von X" und einem höheren Preis in Höhe von P".

Empirische Studien bestätigen die Existenz dieser externen Effekte im F\&E-Bereich. So zeigt Griliches (1992) in einem Überblick über 17 empirische Studien von 1958-1991, dass alle Studien im folgenden Ergebnis übereinstimmen: Die sozialen Erträge von F\&E in Landwirtschaft und Industrie liegen deutlich über den privaten Erträgen. Es existieren also erhebliche externe Effekte von F\&E. ${ }^{32}$ Der Überblick über 15 empirische Untersuchungen im Zeitraum von 1982-1991 von Geroski (1995) weist auf die Existenz von positiven externen Effekten ebenfalls im Bereich der die F\&E-Tätigkeit beinhaltende Innovationstätigkeit hin, wobei das Ausmaß der Spillover-Effekte sehr stark von der betrachteten Branche abhängt und teilweise nur gering ausfällt. ${ }^{33}$ Schließlich untersucht Nordhaus (2004) die Aneignung der Innovationserträge durch innovativ tätige Unternehmer und kommt zu dem Ergebnis, dass Unternehmen nur in der Lage sind, sich 2,2 \% der durch ihre Innovationen entstehenden sozialen Erträge anzueignen. ${ }^{34}$

Auch für den Einfluss der internationalen Innovationstätigkeit auf die nationale Innovationstätigkeit gibt es empirische Untersuchungen, die auf positive externe Effekte hinweisen. Dabei wurde die Innovationsaktivität jeweils mit Hil-

31 Vgl. Fritsch et al. (2003), S. 96 f., für eine generelle wohlfahrtsökonomische Analyse positiver externer Effekte. Vgl. weiterhin Grossmann (2007), Fn. 1, S. 892; Arrow (1962b), S. 619.

32 Vgl. Griliches (1992), S. $43 \mathrm{f}$.

33 Vgl. Geroski (1995), S. $114 \mathrm{ff}$.

34 Vgl. Nordhaus (2004), S. 34. 
fe der Patentanzahl gemessen. Untersuchungsgegenstand von Bottazzi und Peri (2007) ist die Innovationstätigkeit von 15 OECD-Staaten von 1973-1999. ${ }^{35}$ Griffith et. al. (2006) verwenden ein Panel von 188 an der Londoner Börse gelisteten britischen Unternehmen, die Daten decken den Zeitraum von 1990-2000 ab. $^{36}$

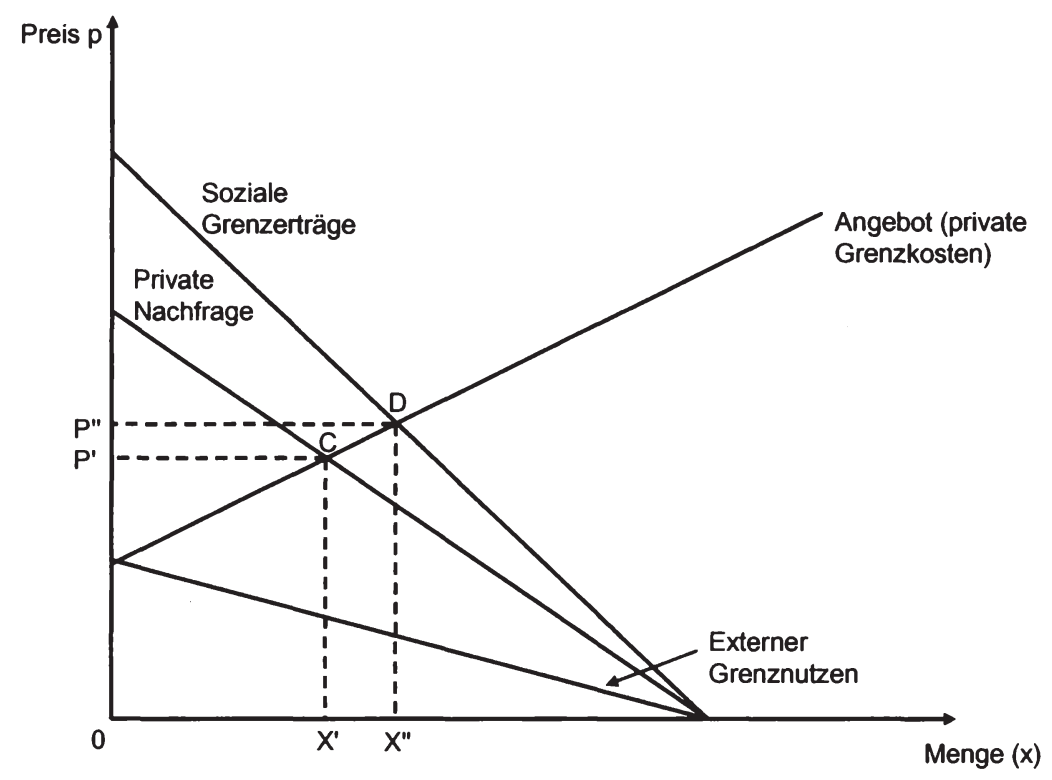

Abbildung 4: Allokatives Marktversagen bei Vorliegen eines positiven externen Effekts (Eigene Darstellung nach Fritsch et al. (2003), S. 123).

\subsubsection{F\&E und Innovationen als öffentliche Güter}

Die mit F\&E einhergehenden positiven externen Effekte führen dazu, dass in der Literatur die einhellige Meinung vorherrscht, F\&E und Inventionen als öffentliche Güter zu bezeichnen. Denn die Ausschließbarkeit anderer Akteure vom Konsum kann meist nicht gewährleistet werden und gleichzeitig besteht NichtRivalität im Konsum. ${ }^{37}$ Dieses Argument gilt in besonderem Maße für die Grundlagenforschung, die zu einer Erhöhung der Faktorproduktivität insgesamt

35 Vgl. Bottazzi und Peri (2007).

36 Vgl. Griffith et al. (2006).

37 Vgl. Arrow (1962b), S. 614 ff.; Romer (1990), S. 74; Hujer und Radić (2005), S. 565 f.; Hartig (1990), S. 12 f. 
führt und als vollkommen öffentliches Gut angesehen werden kann. Dennoch stellt die Grundlagenforschung aber kein freies Gut dar, da ein Unternehmen oder eine Forschungseinrichtung in Infrastruktur, Institutionen, Humankapital und Netzwerke investieren muss, um Erkenntnisse der Grundlagenforschung verstehen und anwenden $\mathrm{zu}$ können. ${ }^{38}$

Trotz des Öffentlichen-Guts-Charakters der Ideen sind jedoch die innovativen Produkte und Produktionsverfahren, die auf den Ideen basieren, durch ,property rights" wie Patente größtenteils bzw. für eine gewisse Zeit ausschließbar. ${ }^{39}$ Da der Produktion von Innovationen fast immer ein hoher Fixkostenblock für F\&E vorausgeht (u. a. bedingt durch Investitionen in Humankapital und Ausrüstung), wäre es allerdings verlustbringend für das Unternehmen, den Preis des innovativen Produktes den Grenzkosten seiner Produktion gleichzusetzen. Stattdessen werden Unternehmen nur dann in den Markt eintreten und mit Hilfe von F\&E Ideen für neue Produkte und Produktionsverfahren entwickeln, wenn die Möglichkeit der Gewinnerzielung besteht, also keine vollkommene Konkurrenz auf dem Markt herrscht. Durchsetzbare ,property rights“, die den Innovatoren ein zumindest zeitweiliges Monopol für ihre Ideen sichern, lösen das Problem des allokativen Marktversagens und sind somit ein wichtiger Anreiz für die Schaffung von Innovationen und damit zusammenhängend für das Wirtschaftswachstum. ${ }^{40}$

38 Vgl. Hartig (1990), S. 14 f.; Callon (1994), S. 399 ff.; Pavitt (2001), S. 764 f.; Oberender (1987), S. 136 f.; Arrow (1962b), S. 618 f.; Nelson (1959), 298 ff. Vgl. Callon (1994), S. 397 ff., für eine Diskussion, unter welchen Umständen bei Grundlagenforschung (bei ihm als „science“ bezeichnet) die Ausschließbarkeit von der Anwendung teilweise gewährleistet werden kann, so dass es sich nur um ein quasi-öffentliches Gut handelt. Vgl. Dasgupta und David (1985) für eine Diskussion der Abgrenzungsmöglichkeiten zwischen Grundlagenforschung (ebenfalls als „science“ bezeichnet) und angewandter Forschung (,technology“). - Interessanterweise zeigt Pavitt (2001), dass es trotz des Öffentlichen-Guts-Charakters der Grundlagenforschung kein Free-RidingVerhalten in dem Sinne gibt, dass sich Länder komplett aus der Grundlagenforschung zurückziehen. Sowohl die westlichen Industrieländer als auch die asiatischen Schwellenländer investieren in den 1980ern und 1990ern stark in die Grundlagenforschung. Der Autor begründet dieses den ökonomischen Vorhersagen widersprechende Phänomen damit, dass anscheinend doch einige mit der Grundlagenforschung zusammenhängende Nutzenzuwächse im Land verbleiben, diese also kein vollkommen öffentliches Gut ist, vgl. Pavitt (2001), 764 ff.

39 Vgl. Geroski (1995), S. 93.

40 Vgl. Arrow (1962b), S. 616 f.; Jones (2002), S. 80 ff.; Dasgupta (1987), S. 14; Metcalfe (1995), S. 419 ff. Die Nutzen durch ein zeitweiliges Monopol sind allerdings gegenüber den mit jedem monopolistischen Markt einhergehenden Gefahren abzuwägen, zu denen die Durchsetzung des hohen Monopolpreises, die Erzielung der Monopolrente durch den Unternehmer und eine deutlich verringerte Konsumentenrente im Vergleich zur Situation der vollständigen Konkurrenz zählen, vgl. Klodt (1995), S. 27 ff. 
Es ist allerdings aus mehreren Gründen zweifelhaft, dass „property rights“ immer vollständig durchsetzbar sind. Erstens können Arbeitskräfte ihren Arbeitgeber wechseln und ihre Erfahrung und Informationen bei ihrem neuen Arbeitgeber mit in das Unternehmen einbringen. Zweitens können sich Unternehmen bei ihrer eigenen Innovationstätigkeit in einem gewissen Ausmaß von den innovativen Produkten der Konkurrenz inspirieren lassen, sofern sie - analog zur obigen Argumentation für die Grundlagenforschung - die nötigen Investitionen in Institutionen, Ausrüstung und Humankapital getätigt haben. Drittens legen Patente teilweise genug Informationen über das zu schützende Projekt offen, so dass die Imitation durch die Konkurrenz paradoxerweise gerade dadurch gesteigert wird. Und viertens können gar nicht alle Innovationen mit Hilfe von ,property rights" geschützt werden. Deshalb variiert der Patentierungsgrad je nach Branche stark. $^{41}$

\subsubsection{Unvollkommene Information}

Eine vollständige Durchsetzung der „property rights“ - sofern sie denn möglich ist - löst zwar das Problem des allokativen Marktversagens, doch bietet sie keine Lösung für den zweiten angesprochenen Grund für die Investitionszurückhaltung der Unternehmen im Bereich Innovationen und F\&E. Neben den bereits erläuterten externen Effekten stellt der unsichere Investitionserfolg ein Problem für die Unternehmen dar, was als eine Form von unvollkommener Information bezeichnet werden kann. Unternehmer scheuen den Kapitalaufwand für Innovationen bzw. F\&E in besonderem Maße, weil Investitionen in die Innovationstätigkeit generell mit einem höheren Risiko verbunden sind als andere Investitionen. Die erste Unsicherheit besteht darin, ob im Rahmen der F\&E-Tätigkeit überhaupt nützliche Ergebnisse erzielt werden (technologische Unsicherheit). Die zweite Unsicherheit besteht darin, ob Produktinnovationen, die auf den Markt gebracht werden, von diesem auch angenommen werden (nachfrageseitige Unsicherheit). ${ }^{42}$ Bei Übertragung dieser Argumentation auf Prozessinnovationen stellt diese Unsicherheit die tatsächliche Anwendung der Innovation im Produktionsprozess innerhalb des Unternehmens dar.

Im Allgemeinen wird davon ausgegangen, dass Wirtschaftssubjekte risikoavers sind. ${ }^{43}$ Anhand der Entscheidung eines Unternehmers zwischen zwei In-

41 Vgl. Brusoni et al. (2006), S. 9; Geroski (1995), S. 103 und S. 118 f.; Dasgupta und David (1985), S. 22.

42 Vgl. Geroski (1995), S. 92; Hujer und Radić (2005), S. 565 f.; Hartig (1990), S. 14; Issing (1979), S. 35; Metcalfe (1995), S. 412 f.

43 Inwiefern die Risikoeinstellung von Unternehmern $\mathrm{zu}$ anderen Wirtschaftssubjekten abweicht und Unternehmer als weniger risikoavers als andere Wirtschaftssubjekte angesehen werden können, wird in Abschnitt 5.5 ausführlicher diskutiert. Ohne den Ergebnissen vorzugreifen, ist es kein Paradoxon, Unternehmer als relativ risikofreudig im Vergleich zu anderen Wirtschaftssubjekten, aber als absolut risikoavers zu beschreiben. 
vestitionsprojekten, die mit unterschiedlichem Risiko behaftet sind, können die Konsequenzen für die Investitionsentscheidungen aufgezeigt werden.

Angenommen, ein Investitionsprojekt $\mathrm{A}$ führt zu einem sicheren Ertrag von $(1 / 2 x+1 / 2 y)$, während ein Investitionsprojekt B mit einer Wahrscheinlichkeit von $50 \%$ zu einem Ertrag in Höhe von $x$ und mit einer Wahrscheinlichkeit von $50 \%$ zu einem Ertrag in Höhe von y führt. Wenn der Unternehmer risikoneutral ist, ist er zwischen den beiden Investitionsprojekten indifferent, da der Erwartungswert des Ertrags aus beiden Investitionsprojekten gleich ist. Ist der Unternehmer aber risikoavers, so hat er eine individuelle Nutzenfunktion mit abnehmendem Grenznutzen des Einkommens, was einem konkaven Kurvenverlauf der in Abbildung 5 dargestellten Nutzenkurve entspricht.

Die Annahme einer konkaven Nutzenfunktion für mögliche Gewinne bzw. Einkommenszuwächse ist seit Ende der 1970er Jahre ausgehend von den wegweisenden Arbeiten von Kahneman und Tversky weit verbreitet und wurde in zahlreichen empirischen Studien bestätigt. ${ }^{44}$ Der risikoaverse Unternehmer wird somit das Investitionsprojekt A dem Investitionsprojekt B vorziehen, weil der Erwartungswert des Nutzens bei Projekt A größer ist als bei Projekt B (vgl. Abbildung 5): ${ }^{45}$

$$
u_{A}=u\left(\frac{1}{2} x+\frac{1}{2} y\right)>u_{B}=u\left(\frac{1}{2} u(x)+\frac{1}{2} u(y)\right)
$$

Bei Anwendung dieses Beispiels auf die Durchführung von Innovationsprojekten, welche annahmegemäß mit einem höheren Risiko als normale Investitionsprojekte verbunden sind, wird deutlich, dass ein Unternehmer das nichtinnovative Investitionsprojekt mit sicherem Ertrag (Investitionsprojekt A) dem innovativen Investitionsprojekt mit unsicherem Ertrag (Investitionsprojekt B) vorzieht.

Bezogen auf die Gesamtheit der Volkswirtschaft gleichen sich durch die Diversifikation die Risiken der einzelnen Investitionsprojekte aus. Da die Risikoeinstellung der Unternehmer von der gesamtwirtschaftlich optimalen Risikoeinstellung abweicht und die Unternehmer somit risikoscheuer sind als der Staat, könnte aufgrund dieses Diversifikationseffektes staatliche Unterstützung für Innovationsprojekte sinnvoll sein. Denn die reinen Marktprozesse führen bei einem risikoaversen Investitionsverhalten zu einer gesamtgesellschaftlichen Unterinvestition in F\&E bzw. Innovation. ${ }^{46}$

44 Für das theoretische Modell und einen Überblick zu entsprechenden empirischen Studien, vgl. Tversky und Kahneman (1986), S. S258.

45 Vgl. Klodt (1995), S. 15 f.

46 Vgl. Klodt (1995), S. 14; Dasgupta (1987), S. 19; Fölster (1991), S. 27. Zu beachten ist hier allerdings der Spezialfall, wenn risikoneutrale Unternehmer durch die Inanspruchnahme staatlicher Fördermittel von möglichen Gewinnen allein profitieren, aber mögli- 


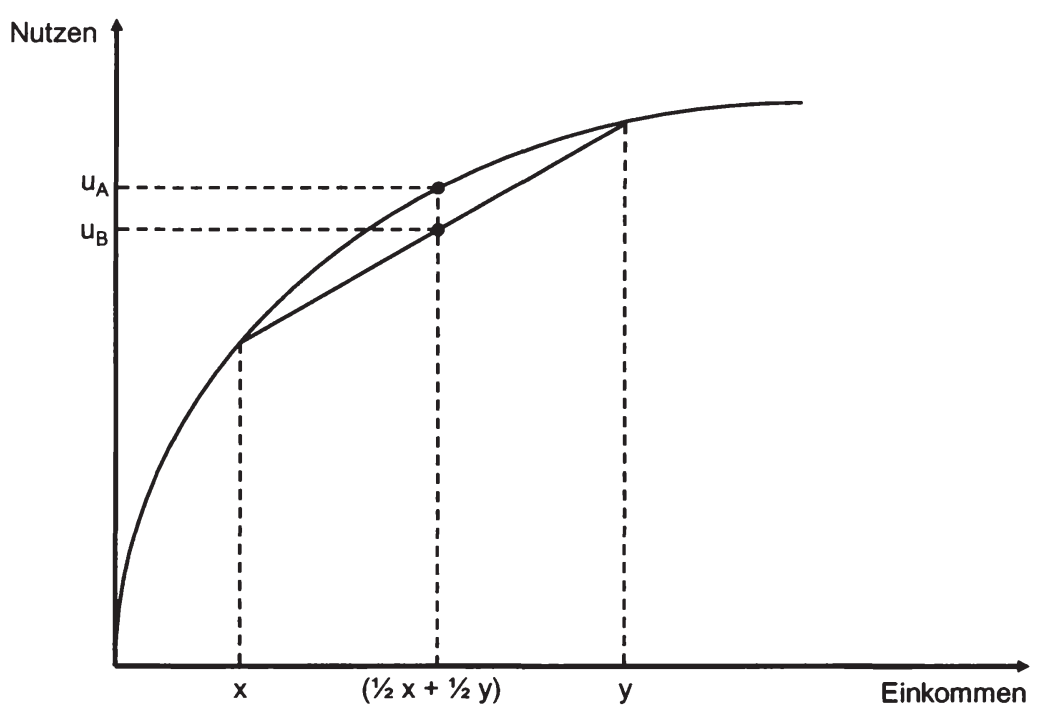

Abbildung 5: Nutzenbewertung von riskanten Investitionsprojekten (Eigene Darstellung nach Klodt (1995), S. 16).

Aber auch für den Fall, dass sich Unternehmer trotz Risikoaversion für die Durchführung von riskanten Innovationsprojekten entschieden haben, treffen sie auf Schwierigkeiten, wenn sie auf die Suche nach externen Kapitalgebern für ihre Innovationsprojekte angewiesen sind. Für die Schwierigkeit der Suche nach externem Kapital gibt es vier Gründe, die im Folgenden erläutert werden.

Erstens liegt eine Informationsasymmetrie im Sinne einer Adversen Selektion (eine Form der in Abschnitt 3.1.3 beschriebenen Prinzipal-Agent-Beziehung) vor. Das bedeutet, dass die Kapitalgeber vor Abschluss eines Vertrags zur Kapitalüberlassung die Erfolgsaussichten der Investitionsprojekte im Allgemeinen schlechter beurteilen können, da sie über weniger Informationen als die Unternehmer selbst verfügen. Somit könnte ein Anreiz für diejenigen Unternehmer, deren Investitionsprojekte relativ geringe Erfolgsaussichten haben, darin bestehen, sich opportunistisch zu verhalten und in besonderem Maße externes Kapital

che Verluste aus riskanten Innovationsprojekten nur teilweise tragen müssen, z. B. weil die Möglichkeit der Insolvenz durch eine unvollständige Haftung für die Verbindlichkeiten im Falle des Scheiterns des Projektes mit einkalkuliert wird. In diesem Fall liegt der private Erwartungswert des Ertrags aus dem Investitionsprojekt nämlich über dem sozialen Erwartungswert und es kommt zu einer gesamtgesellschaftlichen Überinvestition in F\&E; vgl. Klodt (1995), S. 19 f. bzw. Sappington (1991), S. 58 f., mit einer ähnlichen Argumentation in Bezug auf die Kreditvergabe von Banken für risikoreiche Investitionsprojekte. 
für diese Projekte zu beantragen, um das Risiko nicht allein zu tragen. ${ }^{47}$ Die bestehende Informationsasymmetrie wird noch dadurch verstärkt, dass die Unternehmer nicht alle Details ihres Vorhabens bereitwillig preisgeben, sondern ein strategisches Interesse daran haben, bestimmte Informationen bzgl. ihres Innovationsvorhabens für sich zu behalten, damit die Konkurrenz nicht von ihren Ideen erfährt. Denn sobald derartige Informationen preisgegeben sind, können sie kostenlos repliziert und weitergegeben werden, weswegen dem innovierenden Unternehmen sehr daran gelegen sein dürfte, möglichst keine Informationen weiterzugeben, bevor die Innovation am Markt realisiert wurde. ${ }^{48}$ Für den Fall, dass trotzdem eine Kreditbeziehung aufgenommen wird, entstehen weitere Anreizprobleme durch diese Informationsasymmetrie zwischen Kapitalgeber und -nehmer in Form von ,moral hazard“". Diese Probleme werden ausführlicher in Kapitel 4 am Beispiel der Innovationsförderung und der Anreizprobleme zwischen Fördermittel vergebender Institution und Fördermittel empfangendem Unternehmer erläutert.

Der zweite Grund für die Schwierigkeit, externe Kapitalgeber für die Finanzierung zu finden, liegt darin begründet, dass Kapitalgeber generell vor der Finanzierung von Projekten mit hohen Risiken, wie Innovationsprojekten, zurückschrecken. ${ }^{49}$ Begründen lässt sich dies analog zur oben skizzierten Risikoaversion der Unternehmer. Dieser Effekt wird noch dadurch verstärkt, dass bei Innovationsprojekten meist zu wenig Sachanlagen angeschafft werden, die dem Kapitalgeber als Sicherheit dienen können, da der Großteil der Ausgaben für die Entlohnung der Wissenschaftler und Entwickler anfällt und das Resultat des Innovationsprozesses die Erhöhung des Wissensstands innerhalb des Unternehmens einen immateriellen Vermögenswert darstellt. ${ }^{50}$ Deswegen sind interne Rücklagen (Eigenfinanzierung), wie viele empirische Studien zeigen, die wichtigste Finanzierungsquelle für F\&E- und Innovationsprojekte. ${ }^{51}$

Drittens unterliegen kleine und mittlere Unternehmen (KMU) speziellen Budgetbeschränkungen, da sie nur wenige Investitionsprojekte durchführen und somit im Gegensatz zu Großunternehmen keine Risikodiversifizierung betreiben können und außerdem in der Regel höhere Zinsen zahlen müssen, weil sie ihren

47 Bezogen auf allgemeine Unternehmensfinanzierung gibt es in der Literatur vereinzelt auch eine gegenteilige Meinung. So könnten die Kreditgeber besser über die Erfolgschancen eines Projektes informiert sein als die Kredit beantragenden Unternehmen, vgl. Parker (2007), S. 39 f.

48 Vgl. Hall (2002), S. 38; Piga und Atzeni (2007), S. 153; Mohnen et al. (2008), S. 1. Vgl. für die theoretische Modellierung Bhattacharya und Ritter (1983).

49 So zeigen Canepa und Stoneman (2002), dass mit höherem Risiko behaftete Industrien stärker unter Finanzierungsbeschränkungen leiden.

50 Vgl. Hall (2002), S. 36 ff.; Piga und Atzeni (2007), S. 169; Mohnen et al. (2008), S. 2.

51 Vgl. Hall (2002), S. 44 f.; Piga und Atzeni (2007), S. 151 ff.; Mohnen et al. (2008), S. $1 \mathrm{f}$. 
Kreditgebern weniger Sicherheiten bieten können. ${ }^{52}$ Deswegen greifen KMU im Vergleich zu Großunternehmen in einem höheren Ausmaß auf interne Rücklagen als auf kurzfristige Kredite zur Finanzierung ihrer F\&E- und Innovationsprojekte zurück. ${ }^{53}$

Viertens werden externe Kapitalgeber ihre Mittel eher Unternehmen anvertrauen, die Erfahrung aus der Vergangenheit in der Entwicklung und Durchführung von Innovationen vorweisen können. Indikatoren für diese Erfahrung können das Ausmaß der F\&E- und die Innovationstätigkeit des Unternehmens in der Vergangenheit und Gegenwart sein. So finden Piga und Atzeni (2007) in einer empirischen Untersuchung des F\&E-Verhaltens italienischer Unternehmen des Verarbeitenden Gewerbes heraus, dass Unternehmen mit generell niedriger F\&E-Tätigkeit seltener Kredite beantragen als Unternehmen mit generell hoher F\&E-Tätigkeit, und wenn sie es tun, werden ihre Kreditanträge eher abgelehnt. Außerdem hängt für Unternehmen mit niedriger F\&E-Tätigkeit die Wahrscheinlichkeit der Ablehnung ihres Kreditantrags positiv mit dem Ausmaß ihrer F\&ETätigkeit zusammen, was die Autoren der oben beschriebenen unzureichenden Absicherung des Kredits durch Sachanlagen zuschreiben. Dagegen besteht für Unternehmen mit generell hoher F\&E-Tätigkeit kein signifikanter Zusammenhang zwischen F\&E-Tätigkeit und Wahrscheinlichkeit der Kreditablehnung. ${ }^{54}$ In Bezug auf die Finanzierung durch Venture Capital-Fonds, die für die F\&EFinanzierung im Laufe der Zeit immer wichtiger werden, gibt es ähnliche Ergebnisse. Empirische Studien belegen, dass Venture Capital-Fonds gezielt in diejenigen Unternehmen investieren, die eine hohe Patentanzahl aufweisen. ${ }^{55} \mathrm{Da}$ Patente einen Indikator für die F\&E-Erfahrung eines Unternehmens darstellen, deuten auch diese Ergebnisse darauf hin, dass Erfahrung in F\&E- und Innovationstätigkeit als ein positives Signal für den Kapitalgeber dienen kann und dass gerade die Unternehmen mit wenig Erfahrung in der Innovationstätigkeit in der Vergangenheit unter Kreditbeschränkungen leiden, wenn sie Mittel für ein Innovationsprojekt aufbringen müssen. Das Humankapital, das in diesen Unternehmen vorhanden ist, wird also für die Innovationsherstellung in der Gesellschaft systematisch zu wenig genutzt.

52 Vgl. Arrow (1962b), S. 616; Klodt (1995), S. 16 f.; Acs und Audretsch (2005), S. 17. Diese Problematik wurde durch die Eigenkapitalvorschriften des Basler Ausschusses für Bankenaufsicht (unter dem Begriff Basel II bekannt) noch verstärkt. Informationen über Basel II finden sich auf den Internetseiten http://www.bundesbank.de/bankenaufsicht/ bankenaufsicht_basel.php und http://www.basel-ii.info/, zugegriffen am 19.03.2008.

53 Vgl. Schröder und Steiger (1996), S. 115. Vgl. Martínez-Ros und Tribó (2000) für einen Überblick über empirische Studien zu den Finanzierungsquellen von KMU für F\&EProjekte und Magri (2007) für entsprechende empirische Belege für italienische KMU.

54 Vgl. Piga und Atzeni (2007). In der Gruppe der Unternehmen mit hoher F\&E-Tätigkeit befinden sich diejenigen Unternehmen, deren F\&E-Tätigkeit im obersten Quintil der betrachteten Gesamtheit liegt.

Vgl. Mohnen et al. (2008), S. 9, mit einem Überblick. 
Die Ausführungen dieses Abschnitts machen deutlich, dass Unternehmen mit besonderen Schwierigkeiten zu kämpfen haben, wenn sie externes Kapital für ihre Innovationsprojekte benötigen. Die Kapitalfindung wird umso schwieriger bzw. die Risikoprämie steigt umso stärker, je weniger Informationen der Unternehmer über sein Projekt offen legen möchte (Problematik der adversen Selektion), je kleiner das Unternehmen ist (mangelnde Diversifizierungsmöglichkeit), je risikoreicher das Innovationsprojekt ist und je weniger Erfahrung das Unternehmen in diesem Bereich hat, weil es sich in der Vergangenheit nur gering im Bereich F\&E/Innovation engagiert hat. Die Voraussetzung dafür, dass ein Unternehmer trotzdem versucht, solch ein risikoreiches Innovationsprojekt umzusetzen, ist ein gewisses $\mathrm{Ma} ß$ an Risikofreudigkeit bzw. eine vergleichsweise geringer ausgeprägte Risikoaversion.

\subsubsection{Gefahr einer Überinvestition in die Innovationstätigkeit}

Die vorigen Abschnitte befassen sich mit verschiedenen Formen des Marktversagens, die eine gesamtgesellschaftliche Unterinvestition in die Innovationstätigkeit hervorbringen können. Vereinzelt finden sich in der Literatur aber auch modelltheoretische Ergebnisse eines möglichen Marktversagens im Sinne einer privaten Überinvestition in die Innovationstätigkeit bedingt durch Parallelforschung, Patentrennen oder „business stealing“. Allen diesen Modellen liegt die Möglichkeit der Ausschließbarkeit der Konkurrenz von den eigenen Forschungsergebnissen zugrunde.

Die Argumentation bzgl. eines Marktversagens durch Parallelforschung basiert darauf, dass ein Innovatorunternehmen als Monopolist eine Innovationsrente im Markt erzielen kann. Dadurch besteht für andere Unternehmen ein Anreiz, selber F\&E zu betreiben, um Innovationen zu entwickeln und eine Monopolstellung zu erobern. Dasgupta und Stiglitz (1980) zeigen, dass - unter den Annahmen vollkommener Konkurrenz und identischer technologischer Innovation für jedes Unternehmen - genau so viele Unternehmen auf den Markt drängen und Innovationstätigkeit durchführen, bis die gesamtwirtschaftlichen Innovationserträge durch die Innovationskosten verbraucht sind. Die gesamtwirtschaftliche Innovationsrente, die der Innovationsrente des Monopolisten entspricht, wird vollständig aufgezehrt. Es liegt eine Überinvestition in F\&E im Vergleich zum gesamtgesellschaftlichen Optimum vor. Denn dadurch dass sich die Unternehmensanstrengungen der F\&E inhaltlich überlappen, ist die Rate des technischen Fortschritts zu gering und es kommt zu einem ineffizienten Ressourceneinsatz. ${ }^{56}$

Eine ähnliche Argumentation liegt einer Modellvariante von Dasgupta und Stiglitz (1980) zugrunde, die eine gesamtgesellschaftliche Überinvestition in die Innovationstätigkeit aufgrund von Patentrennen beschreibt. Unternehmen konkurrieren miteinander um die Entwicklung einer Innovation. Je näher in der $\mathrm{Zu}$ -

56 Vgl. Dasgupta und Stiglitz (1980), S. 279 f. 
kunft die Innovation erfolgt, desto höher ist der Ertrag einer Innovation und desto höher sind aber auch deren Entwicklungskosten. Es gibt einen gesamtwirtschaftlich optimalen Zeitpunkt für die Innovation, bei der die Differenz zwischen Erträgen und Kosten, die gesamtwirtschaftliche Innovationsrente, maximal ist. Da die Unternehmen aber miteinander im Wettbewerb stehen, haben sie einen Anreiz, ihre eigene Entwicklung zu beschleunigen, um ihre Innovation vor ihren Konkurrenten auf den Markt zu bringen. Denn nur dasjenige Unternehmen mit der schnellsten Invention kann sich das Patent sichern und auf diese Weise durch die vollständig durchgesetzten ,property rights“ eine Monopolrente für das innovative Produkt erhalten. Unter bestimmten Annahmen (freier Marktzutritt für Wettbewerber, fixe Kosten des Entwicklungsprozesses) wird der Wettbewerb solange fortgeführt, bis die gesamte Innovationsrente durch die gestiegenen Innovationskosten aufgezehrt wird und der tatsächliche Innovationszeitpunkt deutlich vor dem gesamtwirtschaftlich optimalen Zeitpunkt liegt. ${ }^{57}$

In einem ähnlichen Modell der Konkurrenz zwischen Forschern, welches sich aber auch auf innovative Unternehmen anwenden lässt, zeigen Dasgupta und Maskin (1987) außerdem, dass die Akteure eher in stark risikoreiche F\&EProjekte investieren, weil sie so ihre Chancen erhöhen, vor der Konkurrenz bahnbrechende Entdeckungen zu machen, die sich in lukrative innovative Produkte umsetzen lassen. ${ }^{58}$ Voraussetzung dieses Ergebnisses ist allerdings die eher unrealistische Annahme der vollkommenen Risikoneutralität der Forscher bzw. Unternehmer.

Unter Beibehaltung der Annahme einer Monopolstruktur auf den Absatzmärkten ist eine gesamtgesellschaftliche Überinvestition in die Innovationstätigkeit auch dann möglich, wenn im Sinne des Schumpeter'schen Prozesses der schöpferischen Zerstörung (vgl. Kapitel 5.6.3) alte Innovatoren durch neue Innovatoren als Monopolisten abgelöst werden, wie Aghion und Howitt (1992) modelltheoretisch zeigen. Innovationen werden hier vordringlich als Verbesserung alter Produkte und nicht als Einführung vollkommen neuer Produkte gesehen. Da die Kunden zu den verbesserten neuen Produkten greifen, eignen sich die neuen Innovatoren Teile der alten Innovationsrenten an (,business stealing"). Da die neuen Innovatoren diesen externen Effekt des Rentenverlustes der alten Innovatoren nicht in ihre Kalkulation mit einbeziehen, ist das Ausmaß ihrer Innovationstätigkeit im Vergleich zum gesamtgesellschaftlichen Optimum zu hoch. ${ }^{59}$

Zusammenfassend ist in Bezug auf die modelltheoretischen Ergebnisse festzuhalten, welche ein mögliches Marktversagen durch Überinvestition in die Innovationstätigkeit beschreiben, dass sie sich auf sehr strikte Annahmen stüt-

57 Vgl. Dasgupta und Stiglitz (1980), S. 284 ff.; Thiel (1988), S. $22 \mathrm{ff}$.

58 Vgl. Dasgupta und Maskin (1987). Vgl. auch das Vorläufermodell bei Dasgupta und Stiglitz (1980), S. 283 f.

59 Vgl. Aghion und Howitt (1992). 
zen, wie z. B. die Möglichkeit der vollständigen Ausschließbarkeit der Konkurrenz von den eigenen Forschungsergebnissen. Diese Ergebnisse konnten bislang weder empirisch noch experimentell bestätigt werden, so dass insgesamt gesehen die theoretische und empirische Argumentation für eine Unterinvestition in F\&E bzw. Innovation in der Gesellschaft deutlich überzeugender erscheint. ${ }^{60}$

\subsubsection{Fazit}

Es liegen gewichtige theoretische und empirische Argumente vor, die für ein allokatives Marktversagen in den Bereichen F\&E und Innovation sprechen. So liegen externe Effekte in Bezug auf F\&E bzw. Innovation vor, die dazu führen, dass die gesellschaftliche Investitionstätigkeit in diesem Bereich unter dem gesamtwirtschaftlich optimalen Ausmaß liegt. Der unsichere Innovationserfolg und die damit zusammenhängende unvollkommene Information führen dazu, dass Unternehmer im Vergleich zum gesamtgesellschaftlichen Optimum von sich aus zu wenig Innovationstätigkeit durchführen und außerdem Schwierigkeiten haben, externes Kapital für Innovationsprojekte zu akquirieren. Zur Behebung des allokativen Marktversagens durch den Staat mit Hilfe von Innovationsförderung sollten demzufolge risikoreiche Innovationsprojekte von risikofreudigen Unternehmern gefördert werden, die in Bezug auf Innovation wenig Erfahrung haben. Dabei führt die Vergabe staatlicher Fördermittel nicht nur direkt zu einer verstärkten unternehmerischen Innovationstätigkeit, sondern es ist davon auszugehen, dass eine Förderung als positives Signal für andere externe Kapitalgeber dient, so dass das geförderte Unternehmen in der Zukunft einfacher Kapital für weitere Innovationsprojekte akquirieren kann. ${ }^{61}$

\subsection{Wachstumseffekte als Begründung für Innovationsförderung}

Um Innovationsförderung ökonomisch mit dem Vorliegen von Wachstumseffekten zu rechtfertigen, können die Erkenntnisse der endogenen Wachstumstheorie benutzt werden, in der Innovation und Technologie zu den Bestimmungsfaktoren von Wirtschaftswachstum gehören und positive Effekte auf die Beschäftigung auslösen können. Innovationen sind nicht nur für ein konstantes Wirtschaftswachstum wichtig, sondern treiben die Produktivität und somit die Wettbewerbsfähigkeit von Unternehmen voran und sorgen somit dafür, dass das Niveau des Wachstumspfades angehoben wird und dass gleichzeitig der Wachstumspfad steiler wird, die Wachstumsrate einer Volkswirtschaft bzw. Region

60 Auch in der Literatur wird die Möglichkeit einer gesellschaftlichen Überinvestition eher am Rande behandelt, vgl. Geroski (1995), S. 120.

61 Vgl. Lerner (1999) für eine theoretische und empirische Bestätigung dieser Argumentation am Beispiel des Small Business Innovation Research Program. 
also im Zeitablauf zunimmt. ${ }^{62}$ Es gibt verschiedene endogene Wachstumsmodelle, im Folgenden werden die vier für das Thema Innovationsförderung wichtigsten kurz vorgestellt:

Arrow entwickelte 1962 das Learning-by-doing-Modell, das auf externen Effekten der Investitionen in Sachkapital beruht. Durch Erfahrung in der Produktion entstehen Lerneffekte, die nicht ausschließlich dem investierenden Unternehmen zugute kommen. Alle Unternehmen profitieren von der Gesamtheit der in dieser Volkswirtschaft gemachten Erfahrungen, die Arrow mit Hilfe der kumulierten Güterproduktion der Vergangenheit abbildet. Die existierenden externen Effekte (vgl. Abschnitt 2.3.1) führen dazu, dass die private Investitionsquote unter der gesamtgesellschaftlich optimalen Investitionsquote liegt. ${ }^{63}$

Lucas Jr. (1988) führte in seinem Wachstumsmodell zusätzlich zu den herkömmlichen Produktionsfaktoren Arbeit und Kapital den akkumulierbaren Faktor Humankapital in die Produktionsfunktion ein, welcher neben dem positiven internen Effekt auf die individuelle Produktivität einen positiven externen Effekt auf die Produktivität der anderen beiden Produktionsfaktoren in der Gesellschaft erzeugt. ${ }^{64}$ Lucas Jr. zeigt, dass die effiziente Wachstumsrate des Humankapitals höher ist als die im Konkurrenzgleichgewicht tatsächlich erreichte Wachstumsrate, woraus sich ableiten lässt, dass auch das gesamtwirtschaftliche tatsächliche Wachstum hinter dem optimalen zurückbleibt. ${ }^{65}$ Länder mit unterschiedlichen Anfangsausstattungen des Humankapitals konvergieren $\mathrm{zu}$ langfristig unterschiedlichen Einkommensniveaus, wodurch Lucas Jr. eine Erklärung für permanente Einkommensunterschiede zwischen Industrie- und Entwicklungsländern findet. ${ }^{66}$

Eines der einflussreichsten Modelle im Bereich der endogenen Wachstumstheorie ist das von Romer 1990 entwickelte Produktionsmodell mit drei Sektoren: einem Forschungssektor, einem Sektor für die Produktion von Zwischenprodukten und einem Sektor für die Produktion der Endprodukte, wobei die meisten Unternehmen gleichzeitig in allen drei Sektoren tätig sind. Es gibt vier Inputfaktoren in diesem Modell: Kapital, Arbeit, Humankapital und Stand der Technologie. ${ }^{67}$ Wenn nun ein Unternehmen in F\&E investiert und beispielsweise ein neues Design entwirft, produziert es ein neues Gut, wofür es über den Markt entlohnt wird. Zusätzlich fällt aber ein externer Effekt an, weil das Unternehmen mit seiner F\&E-Tätigkeit den allgemeinen Wissensstock der Gesell-

62 Vgl. Romer (1990), S. 97; Lambooy (2000), S. 106 ff.; Klodt (1995), S. 98; Zagler (2007), S. 821 ff. Vgl. Griliches (1992), S. 29 f., für empirische Belege des positiven Zusammenhangs zwischen F\&E, Produktivität und Wirtschaftswachstum.

63 Vgl. Arrow (1962a).

64 Vgl. Lucas Jr. (1988), S. 17 f.

65 Vgl. Lucas Jr. (1988), S. 23 ff.

66 Vgl. Lucas Jr. (1988), S. 25.

67 Vgl. Romer (1990), S. 78 f. 
schaft erhöht und die Produktivität der Forschung anderer Unternehmen erhöht, wofür es keine Entlohnung über den Markt erhält. ${ }^{68}$ Durch diesen externen Effekt ist anzunehmen, dass zu wenig Humankapital im Forschungssektor eingebunden wird, weil das soziale marginale Produkt des Humankapitals höher ist als das private marginale Produkt, nach welchem sich die Entlohnung des $\mathrm{Hu}$ mankapitals im Forschungssektor richtet. Stattdessen wird zu viel Humankapital in der Produktion der Endprodukte eingesetzt. ${ }^{69}$ Die Wachstumsrate der Volkswirtschaft (g) hängt bei Romer ausschließlich vom Zinssatz (r) und dem Produkt aus Produktivität $(\delta)$ und Humankapital $(\mathrm{H})$ ab:

$$
g=\delta H_{A}=\delta H-\Lambda r^{70}
$$

Die Wachstumsrate nimmt mit steigendem Humankapitalbestand zu. Der negative Einfluss des Zinssatzes erklärt sich dadurch, dass die Kosten einer Innovation in der Gegenwart, die Erträge aber erst in der Zukunft anfallen. ${ }^{71}$ Romer selbst weist auf die Möglichkeit hin, dass eine Subventionierung des Forschungssektors die negativen Effekte der Fehlallokation des Humankapitals abmildern kann, so dass sich die Wachstumsrate der Volkswirtschaft erhöht und die Volkswirtschaft auf einen höheren Wachstumspfad gelangt. ${ }^{72}$

Ein weiteres endogenes Wachstumsmodell, das zur Rechtfertigung der Subventionierung von Innovation herangezogen werden kann, wurde von Grossman und Helpman (1990) entwickelt und besteht aus einer Volkswirtschaft mit zwei Produktionssektoren. ${ }^{73}$ Der Output $X$ der beiden Sektoren $i=1,2$ hängt von den eingesetzten Produktionsfaktoren Land $(\mathrm{T})$ und Arbeit $(\mathrm{L})$ sowie der Produktionsfunktion (F) ab, wobei das Wissen (K) als öffentliches Gut in die Funktion mit eingeht:

$$
X^{i}=K F^{i}\left(T^{i}, L^{i}\right), i=1,2
$$

In Sektor 1 treten bei der Produktion externe Effekte in Form von Wissen (,Knowledge“) auf, welche die Produktivität in beiden Sektoren steigern, ohne dass die diese Effekte generierenden Unternehmen sich die Erträge aneignen können. Da die Unternehmen nur ihre privaten Erträge berücksichtigen, kommt es zu einer suboptimalen Allokation der Ressourcen in Sektor 1 und zu einer geringeren Wachstumsrate als im Wohlfahrtsoptimum. Ein staatlicher Eingriff,

68 Vgl. Romer (1990), S. 84.

69 Vgl. Romer (1990), S. 96.

70 HA stellt das Humankapital dar, das in den Forschungssektor investiert wird. $\Lambda$ ist eine Konstante, vgl. Romer (1990), S. 92.

71 Vgl. Romer (1990), S. 94.

72 Vgl. Romer (1990), S. $96 \mathrm{f}$.

73 Vgl. Grossman und Helpman (1990b). 
z. B. durch eine Subventionierung der Produktion des Sektors 1, kann hier zu Wohlfahrtsverbesserungen führen. ${ }^{74}$

Die Problematik des allokativen Marktversagens kann durch die vollständige Durchsetzung von ,property rights“ behoben werden, wie in Abschnitt 2.3 diskutiert wurde. Selbst bei vollständiger Durchsetzung dieser ,property rights“ zeigt die endogene Wachstumstheorie jedoch, dass in einer Volkswirtschaft zu wenig F\&E angeboten wird, da Ideen bzw. Innovationen neben den erwähnten externen Effekten auf andere Unternehmen auch positive externe Effekte in Bezug auf den Nutzen der Konsumenten und künftiger Generationen ausüben. Denn diese profitieren ebenfalls von den Innovationen, beispielsweise dadurch dass die Preise der innovativen Güter ihre Qualitätsverbesserung nicht vollständig widerspiegeln. ${ }^{75}$ Ihre Zahlungsbereitschaft taucht jedoch erst in der Zukunft auf und wird jedoch von den Unternehmen deswegen in ihrer gegenwärtigen Kalkulation nicht berücksichtigt.

Empirische Untersuchungen unterstützen die Bedeutung von Innovation für das Wirtschaftswachstum. ${ }^{76}$ So findet Jungmittag (2004) empirische Belege dafür, dass Innovation einen positiven Einfluss auf das langfristige Wirtschaftswachstum von 14 EU-Staaten im Zeitraum von 1969-1998 ausübt. ${ }^{77}$ Auch Lederman und Saenz (2005) stellen einen positiven Einfluss von Innovation auf das langfristige Wirtschaftswachstum fest. ${ }^{78}$ Dazu verwenden sie Daten des Patentamts der USA (U.S. Patent and Trademark Office) für 164 Herkunftsländer der Inventoren im Zeitraum von 1963-2000 und des Europäischen Patentamts (EPO) für 172 Herkunftsländer der Inventoren im Zeitraum von 1980-2000. Eine aktuelle Studie von Zagler (2007) für Österreich im Zeitraum von 1976-2000 ergibt ebenfalls einen erheblichen positiven signifikanten Einfluss der Innovationsrate auf die Wachstumsrate. Eine Erhöhung der Innovationsrate um $1 \%$ erhöht die Wachstumsrate um ca. 0,4\%. ${ }^{79}$ Auch für die positive Beziehung zwischen Innovativität und Beschäftigung finden sich empirische Belege, bei-

74 Vgl. Grossman und Helpman (1990b), S. 89. Vgl. für eine ausführlichere modelltheoretische Analyse bei Handel zwischen zwei Ländern mit unterschiedlichen komparativen Vorteilen Grossman und Helpman (1990a).

75 Ein oft zitiertes Beispiel in diesem Zusammenhang ist die Verbesserung der Leistungen von Computern bei gleichzeitig sinkenden Verkaufspreisen, vgl. Griliches (1992), S. 36.

76 Innovation wird in diesen Untersuchungen fast immer mit Hilfe der Patentanzahl gemessen. Die Problematik bei der Messung von Innovation und eine Diskussion der üblicherweise verwendeten Indikatoren wird in Abschnitt 6.4.3 ausführlicher diskutiert.

77 Vgl. Jungmittag (2004), S. 22. Eine detaillierte Untersuchung der wechselseitigen Beeinflussung von Pro-Kopf-Einkommen und Innovativität in Deutschland von 18501913 und 1951-1999 findet sich in Jungmittag und Grupp (2006).

78 Vgl. Lederman und Saenz (2005), S. 23 f.

79 Vgl. Zagler (2007), S. 827. 
spielsweise bei Lachenmaier und Rottmann (2008) für deutsche Unternehmen des Verarbeitenden Gewerbes im Zeitraum 1982-2003. ${ }^{80}$

Die endogene Wachstumstheorie mit ihrer Betonung der großen Bedeutung der Innovativität bzw. des technischen Fortschritts für die langfristigen Wachstumsperspektiven einer Volkswirtschaft dient häufig als Begründung für eine innovationsorientierte Wirtschaftsförderpolitik. ${ }^{81}$ Die ordnungspolitische Begründung des Marktversagens im Innovationsbereich durch das Vorliegen externer Effekte ist dagegen zweitrangig. Auch in Niedersachsen wird auf die wachstumspolitische Argumentation zurückgegriffen, wie an Ziffer 4.3 der NIFPRichtlinie deutlich wird, in der es heißt, dass geförderte Vorhaben „einen Beitrag für den gesamtwirtschaftlichen Innovationsprozess leisten [...]. Das ist insbesondere dann der Fall, wenn zu erwarten ist, dass ein Vorhaben zur Steigerung der Leistungsfähigkeit der niedersächsischen Wirtschaft oder zur Schaffung bzw. Sicherung von Arbeitsplätzen beiträgt. “

Die wachstumspolitische Begründung für Innovationsförderung legt die Förderung von Innovationsprojekten mit einer relativ hohen Erfolgswahrscheinlichkeit und relativ sicheren Wachstums- und Beschäftigungsbeiträgen nahe. Es ist anzunehmen, dass diese Projekte eher von Unternehmen durchgeführt werden, die über Erfahrung mit innovativen Produkten und Prozessen verfügen, also schon in der Vergangenheit innovativ tätig waren. Dass diese Unternehmer Fördermittel für ihre Projekte beantragen, deutet auf eine gewisse Risikoscheu hin. Denn eigentlich müsste es für sie bei den Erfolgsaussichten ihrer Projekte und ihrer Erfahrung im Bereich Innovation möglich sein, Finanzierungsmittel aus anderen Quellen zu erhalten, die aus den zukünftigen Erträgen zurückgezahlt werden müssen. Der Aspekt der Risikoeinstellung von Unternehmern, die sich für Innovationsförderung bewerben, wird in Abschnitt 5.5.5 im Rahmen der Entrepreneurship-Theorie ausführlich behandelt.

Es ist festzustellen, dass aus wachstumspolitischen Überlegungen also eher sichere Innovationsprojekte risikoscheuer Unternehmer, die über ausreichend Erfahrung in der Innovationstätigkeit verfügen, gefördert werden sollten. Denn deren Innovationsprojekte haben hohe Erfolgschancen und tragen so mit hoher Wahrscheinlichkeit zu einer Erhöhung des Wachstums und der Beschäftigung bei.

\subsection{Effizienz, Treffsicherheit und Informationsanforderungen}

Nach Darlegung der beiden verschiedenen wirtschaftspolitischen Begründungsmöglichkeiten für Innovationsförderung soll im Folgenden die Wirkungsweise

80 Vgl. Lachenmaier und Rottmann (2008), S. 267 f.

81 Vgl. Ewers und Brenck (1992), S. 312. 
einer Subvention auf die F\&E- bzw. Innovationstätigkeit graphisch verdeutlicht werden. Daran anschließend werden Effizienz und Treffsicherheit dieses Instruments diskutiert und auf die notwendigen Informationsanforderungen für die beteiligten Akteure eingegangen. Zu diesem Zweck greift dieser Abschnitt auf Abbildung 4 aus Abschnitt 2.3.1 zurück, in der die Auswirkungen der vorliegenden positiven externen Effekte auf die F\&E- und Innovationstätigkeit dargestellt wurde. Eine so genannte Pigou'sche Subvention (zahlbar pro Mengeneinheit der F\&E-Tätigkeit) in Höhe der gesamtgesellschaftlichen Grenzerträge im gesamtgesellschaftlichen Optimum könnte die Unternehmen dazu veranlassen, das gesamtgesellschaftlich optimale Niveau an F\&E bereitzustellen. ${ }^{82}$ In Abbildung 6 verschiebt die Subvention die ursprüngliche Angebotskurve der Unternehmen nach unten, so dass das neue Marktgleichgewicht im Punkt E mit Produktion der gesamtwirtschaftlich optimalen Menge X" erreicht wird.

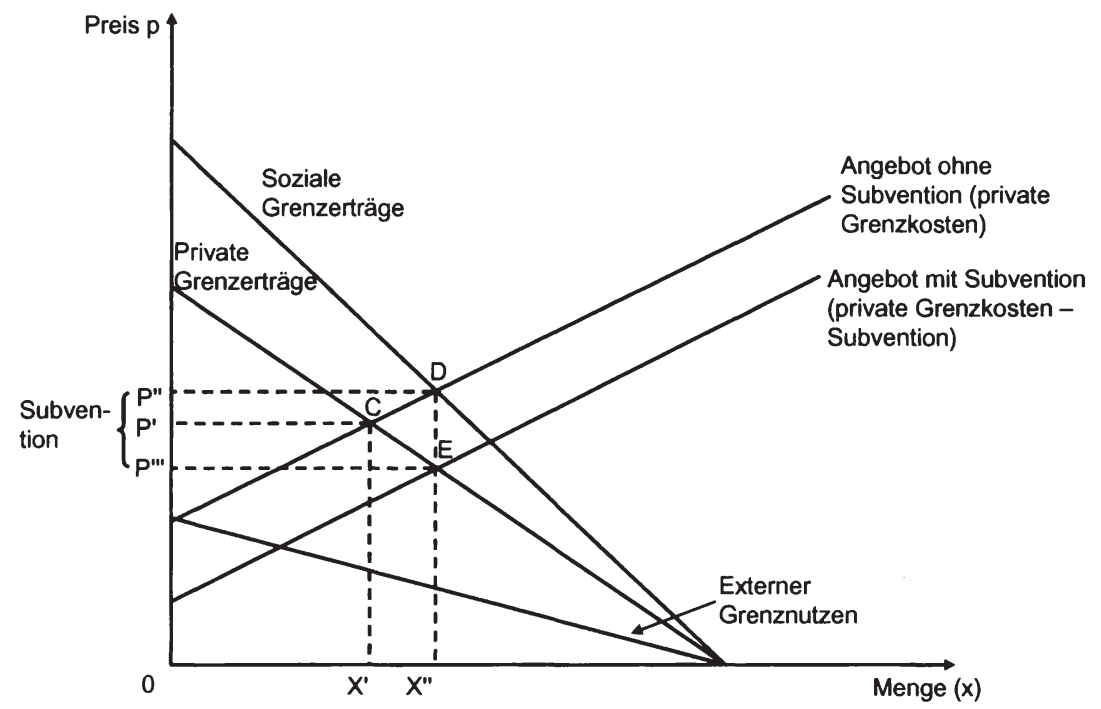

Abbildung 6: Wirkungsweise einer Pigou'schen Subvention (Eigene Darstellung nach Fritsch et al. (2003), S. 123).

Eine Pigou'sche Subvention funktioniert nur unter der Voraussetzung dass der soziale Zusatznutzen durch die Innovationstätigkeit und das gesamtgesellschaftliche Optimum genau bestimmt werden können. Sofern es überhaupt möglich wäre, den sozialen Zusatznutzen zu messen, ist ein immenser Informationsaufwand für die die Subventionshöhe festlegende staatliche Verwaltung erforder- 
lich. ${ }^{83}$ In der Praxis werden deswegen anstelle der Pigou'schen Subventionen Zuschüsse gemäß dem Preis-Standard-Ansatz verwendet. Dieser Ansatz verzichtet auf die Ermittlung eines gesamtgesellschaftlichen Optimums und die Messung des sozialen Zusatznutzens. Stattdessen bestimmt die Politik einen Zielwert für das angestrebte Ausmaß der subventionierten Tätigkeit. Wichtig ist hierbei, dass die Subvention an eine geeignete Bezugsgröße gekoppelt ist, die eng mit dem externen Effekt zusammenhängt. Die Wirkungsweise einer Subvention nach dem Preis-Standard-Ansatz verdeutlicht Abbildung 7. Bei einer Subventionshöhe in Höhe von S (pro Einheit der Externalität) und der Annahme von steigenden Grenzkosten erzeugt das geförderte Unternehmen ein Ausmaß der Externalität in Höhe von N, da in diesem Punkt die Grenzkosten der Erzeugung der Externalität genau der erhaltenen Subvention entsprechen. Je höher der Subventionssatz, desto höher das produzierte Ausmaß der Externalität. ${ }^{84}$

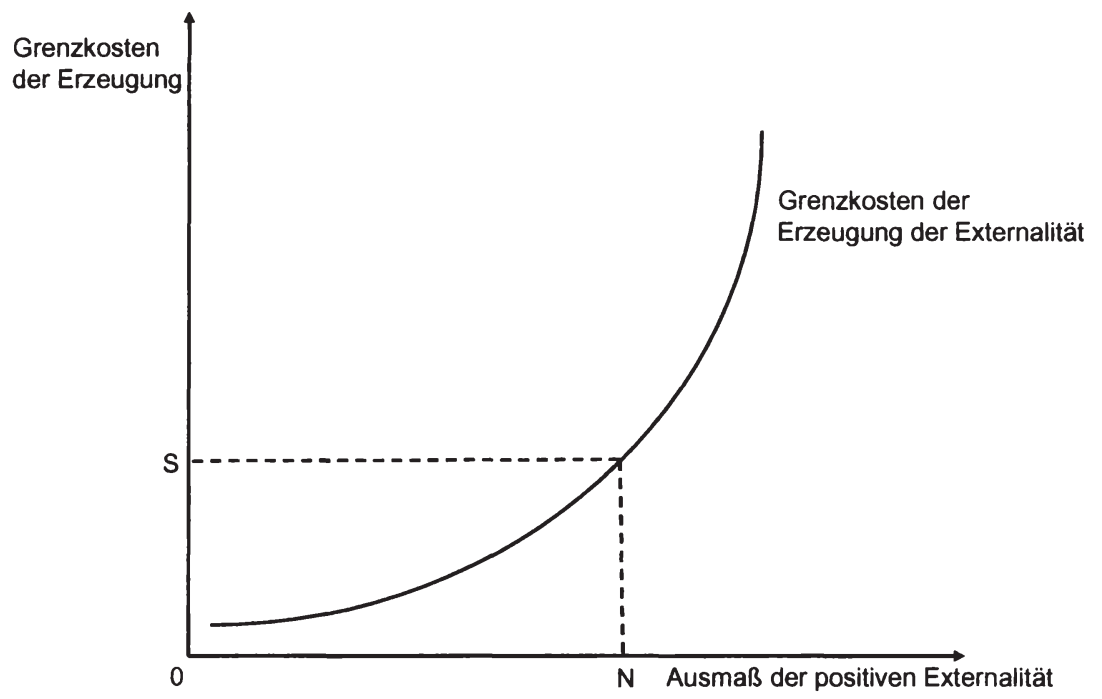

Abbildung 7: Wirkungsweise einer Subvention nach dem Preis-Standard-Ansatz (Eigene Darstellung nach Fritsch et al. (2003), S. 126).

Bei der Bewertung der Effizienz und Treffsicherheit des Instrumentes der Innovationssubvention ergibt sich ein differenziertes Ergebnis. Die Subventionen nach dem Preis-Standard-Ansatz sind statisch effizient, weil die Innovationstätigkeit der Unternehmen erhöht wird, in dem Sinne, dass die Unternehmen mit niedrigeren Grenzkosten der Erzeugung der Externalität ihre Produktion stärker

83 Vgl. Fritsch et al. (2003), S. $124 \mathrm{f}$.

84 Vgl. Fritsch et al. (2003), S. $125 \mathrm{f}$. 
erhöhen als die Unternehmen mit höheren Kosten. Es bestehen weiterhin Anreize zur kontinuierlichen Verbesserung der Innovationstätigkeit im Sinne einer Produktionskostensenkung, so dass auch das Kriterium der dynamischen Effizienz erfüllt ist. Die Treffsicherheit ist dagegen gering, da der Staat zur Festlegung der Subventionshöhe die privaten Kostenkalkulationen der Unternehmen in diesem Bereich kennen muss - eine zugegebenermaßen unrealistische Annahme. ${ }^{85}$ Zusammenfassend ist also festzustellen, dass Innovationsförderung in Form von Zuschüssen zu einer Ausweitung der Innovationstätigkeit führen kann, die die Kriterien der statischen und dynamischen Effizienz erfüllt, aber aufgrund der mangelnden Treffsicherheit nicht systematisch das gesamtgesellschaftliche Optimum der Innovationstätigkeit herstellen kann.

Ein wesentlicher Kritikpunkt am Einsatz der direkten Innovationsförderung ist die staatliche Beeinflussung der Investitionsstruktur, welche auch zu einer Veränderung der Produktionsstruktur und somit der sektoralen Struktur der Volkswirtschaft führt. Nur wenn die für Innovationsförderung verantwortlichen Politiker bzw. Institutionen besser als der Markt über die Erfolgsaussichten und technologischen Möglichkeiten der Innovationen informiert sind, können sie qualifizierte Entscheidungen bzgl. der Auswahl der zu fördernden Sektoren vornehmen und ein Ergebnis für die Gesamtwirtschaft erreichen, das der Situation ohne staatliche Einflussnahme vorzuziehen ist. ${ }^{86}$ Die erheblichen Prognoseschwierigkeiten, die mit einer Vorhersage des Innovationserfolgs und der noch nicht entwickelten Nachfrage nach neuen Produkten einhergehen, betreffen Politiker und die öffentliche Verwaltung aber genauso wie andere Wirtschaftssubjekte. Eine einzelne Institution wie die zuständige Verwaltung kann im Gegensatz zum Entdeckungsmechanismus Markt die Komplexität der miteinander verbundenen Phänomene und Informationen viel schlechter verarbeiten und demzufolge auch schlechtere Einschätzungen vornehmen. ${ }^{87}$

Aus diesen Gründen verwundert es nicht, dass in der Praxis die geförderten so genannten Zukunftstechnologien überall identisch sind, weil alle Verwaltungen dieselben Prioritäten setzen. Dieses Vorgehen ist aus wohlfahrtsökonomischen Überlegungen nicht sinnvoll, weil erstens jedes Land bzw. jede Region andere komparative Standort- und Kostenvorteile aufweist und eine internatio-

85 Vgl. Fritsch et al. (2003), S. $127 \mathrm{ff}$.

86 Vgl. Issing (1979), S. 38 ff.; Zeppernick (1985), S. 74 ff. Auf die Möglichkeit, dass Politik und Verwaltung nicht das gesamtgesellschaftliche Optimum anstreben, sondern Eigeninteressen verfolgen, wird in den Kapiteln 3 und 4 ausführlich eingegangen.

87 Vgl. Schienstock (2000), S. 23; Arrow (1962b), S. 623; Zeppernick (1985), S. 74 f.; Metcalfe (1995), S. 414 f. Ein Beispiel für staatliche Fehleinschätzungen von Technologien ist die Konzentration der deutschen F\&E-Förderung in den 1970er Jahren auf die Erforschung alternativer Energien im Bereich der Kohleverflüssigung als Reaktion auf die erste Ölkrise und die Vernachlässigung anderer alternativer Energien, die sich aber im Laufe der Zeit als viel bedeutsamer herausgestellt haben, vgl. Oberender (1987), S. 130. 
nale technologische Spezialisierung dementsprechend und auch aufgrund von eventuell vorliegender Skaleneffekte folgerichtig wäre. Außerdem kann zweitens ein internationaler Subventionswettlauf entstehen, in dem jedes Land Innovationsförderung zum Schutz der einheimischen Unternehmen betreibt (Protektionismus), damit deren Wettbewerbsfähigkeit nicht unter den subventionierten ausländischen Konkurrenten leidet. ${ }^{88}$

Zusammenfassend ist von einer Festlegung der Förderung auf einzelne Branchen abzusehen, da dieses einer staatlichen Strukturpolitik im Sinne einer Lenkung der Wirtschaftsstruktur entspricht. Eine Bevorzugung bestimmter Branchen kann daher nur mit anderen politischen Zielen begründet werden. Die theoretische Bewertung von Innovationsförderung in Form von Zuschüssen bezieht sich auf generelle Investitionszuschüsse für alle innovativ tätigen Unternehmen. Im Rahmen des NIFP wird aber nur eine Auswahl von bestimmten Innovationsprojekten gefördert. Deswegen ist es zur Abschätzung der Wirkungsweise im Rahmen einer Analyse des Programms unbedingt erforderlich, die institutionelle Struktur, die Auswahlprinzipien und die am Entscheidungsprozess über die Vergabe der Fördermittel beteiligten Akteure zu untersuchen. Zu diesem Zweck nimmt Kapitel 4 dieser Arbeit eine institutionenökonomische Analyse vor.

\subsection{Zusammenfassung der Ergebnisse und Hypothesen}

Das Vorliegen von positiven externen Effekten im Bereich F\&E, das hohe Risiko der Innovationsprojekte und die damit zusammenhängende Investitionszurückhaltung und Finanzierungsschwierigkeiten der Unternehmer stellen ebenso wie die durch Innovation generierten positiven Wachstumseffekte für die Volkswirtschaft gewichtige Argumente für ein staatliches Eingreifen in Form von Innovationsförderung dar. Selbst eine vollständige, gleichwohl unrealistische Durchsetzung der ,property rights“ führt nicht zum gesamtgesellschaftlichen Optimum, wenn externe Effekte in Bezug auf den Nutzen zukünftiger Konsumenten und Unternehmen vorliegen.

Es gibt zwei mögliche Zielgruppen von Innovationsförderung. Die erste Zielgruppe sollte aus allokationspolitischen Gründen, die zweite aus wachstumspolitischen Erwägungen heraus gefördert werden. Die Formulierung von Ziffer 4.3 der NIFP-Richtlinie deutet darauf hin, dass die zweite Gruppe die eigentliche Zielgruppe des NIFP darstellt. Welche Unternehmen bzw. Unternehmer im Rahmen des NIFP tatsächlich gefördert werden, wird in Kapitel 6 anhand der folgenden Hypothesen empirisch untersucht, welche auf der Argumentation der vergangenen Abschnitte aufbauen: 
Hypothese 1: Es werden eher risikoreiche Innovationsprojekte von risikofreudigen Unternehmern, die in Bezug auf Innovation wenig Erfahrung haben, gefördert (allokationspolitische Begründung).

Hypothese 2: Es werden eher sichere Innovationsprojekte risikoscheuer Unternehmer, die über ausreichend Erfahrung die Innovationstätigkeit betreffend verfügen, gefördert (wachstumspolitische Begründung).

Bevor die Hypothesen empirisch überprüft werden, beschreibt Kapitel 3 dieser Arbeit die divergierenden Interessen und Anreize der an Innovationsförderung beteiligten Akteursgruppen mit den sich daraus ergebenden Problemen und institutionellen Lösungsmöglichkeiten aus Sicht der Neuen Institutionenökonomik. In Kapitel 4 werden diese theoretischen Ergebnisse anschließend auf die konkrete institutionelle Ausgestaltung des NIFP und die an diesem Programm beteiligten Akteure angewendet. 


\section{Theorie der Neuen Institutionenökonomik}

„There can be no doubt, that, if power is granted to a body of men, called representatives, they, like any other men, will use their power, not for the advantage of the community, but for their own advantage, if they can. " 89

Das Ziel dieses Kapitels besteht darin, die für die institutionenökonomische Analyse des NIFP nötigen theoretischen Konzepte und Theorien bereitzustellen. Die Ergebnisse dieses Kapitels können dann in Kapitel 4 dazu verwendet werden, Hypothesen bezüglich des Verhaltens der am NIFP beteiligten Akteure aufzustellen. $\mathrm{Zu}$ diesem $\mathrm{Zweck}$ wird zunächst gezeigt, dass ein Innovationsförderprogramm eine Institution darstellt, die die Anreize und das Verhalten der beteiligten Akteure beeinflusst. Um die Mechanismen dieser Institution detailliert zu untersuchen, wird die Neue Institutionenökonomik zu Hilfe genommen, die die Reaktion von Individuen auf Institutionen untersucht. In Abschnitt 3.1 werden die grundlegenden Theorieansätze der Neuen Institutionenökonomik - Informationsasymmetrien, „bounded rationality“, Transaktionskosten sowie PrinzipalAgent-Beziehungen - vorgestellt. $\mathrm{Zu}$ den beteiligten Akteuren zählen erstens die das Innovationsförderprogramm beschließenden Politiker, zweitens die das Programm umsetzenden Bürokraten und drittens die Fördermittel beantragenden Unternehmer. Für die ersten beiden Akteursgruppen gibt es spezielle Theorien der Neuen Institutionenökonomik, die in den Abschnitten 3.2 und 3.3 vorgestellt werden - die Neue Politische Ökonomie und die Theorie der Bürokratie. Abschnitt 3.4 schließt mit einer Zusammenfassung der Ergebnisse.

\subsection{Grundlegende Theorieansätze der Neuen Institutionen- ökonomik}

Die Forschungsrichtung der Neuen Institutionenökonomik untersucht die Entstehung, das Funktionieren und den Wandel von Institutionen. ${ }^{90}$ Es gibt keine einheitliche Definition des Begriffes Institutionen, sondern im Wesentlichen zwei miteinander konkurrierende Definitionen: Institutionen als Spielregeln oder als Ergebnis eines Spiels. ${ }^{91}$

89 Mill (1820), Rn. 79.

90 Die Neue Institutionenökonomik baut auf dem Ende des 19. Jahrhunderts in den USA gegründeten Institutionalismus auf. Die Definition des Institutionsbegriffs in beiden Theorierichtungen ist sehr nahe, abgesehen davon, dass im Institutionalismus das Konzept der Gewohnheiten (, habits“) eine zentrale Rolle spielt, zur Abgrenzung zwischen beiden Theorierichtungen vgl. Hodgson (1998).

91 Vgl. Richter und Furubotn (2003), S. 9. 
Der erste Ansatz, Institutionen als Spielregeln sowohl formeller als auch informeller Natur zu beschreiben, ist in der Literatur weit verbreitet und geht auf Schmoller (1900) zurück, der den Begriff Institution beschreibt als ,eine partielle, bestimmten Zwecken dienende, zu einer selbständigen Entwickelung gelangte Ordnung des Gemeinschaftslebens, welche das feste Gefäß für das Handeln von Generationen, oft von Jahrhunderten und Jahrtausenden abgiebt "92 Als Beispiele nennt er das Eigentum und die Gewerbefreiheit. ${ }^{93}$ Anknüpfend an Schmoller definieren Richter und Furubotn (2003) eine Institution als ,,ein System miteinander verknüpfter, formgebundener (formaler) und formungebundener (informeller) Regeln (Normen) einschließlich der Vorkehrungen zu deren Durchsetzung ".94 Indem Institutionen Ordnung in das alltägliche Leben bringen, regeln sie die zwischenmenschlichen Beziehungen und verringern dabei Unsicherheit. Institutionen schaffen aus Interaktionen zwischen Individuen eine Gruppe, ob es sich im Kleinen um eine Familie oder im Großen um eine nationale Volkswirtschaft handelt. Formgebundene Institutionen werden durch einen oder mehrere soziale Planer bewusst festgelegt und zielgerichtet ausgestaltet, wie z. B. jegliche Form von Gesetzen. Formlose Institutionen entstehen dagegen ungeplant, spontan, durch menschliche Aktion und kulturelle Evolution, wie z. B. Sitten und Gebräuche. ${ }^{95}$

Der zweite Ansatz, Institutionen als Ergebnis eines Spiels zu definieren, wird u. a. von Schotter $(1981,1986)$ vertreten. Im Unterschied zum regelorientierten Erklärungsansatz untersucht Schotter die Konventionen sozialen Verhaltens, die sich evolutorisch entwickeln, um eine Lösung auf sich wiederholende Problemstellungen zu finden. ${ }^{96}$ Für ihn sind soziale Institutionen ,a regularity in social behavior that is agreed to by all members of society "97, welche unter Anwendung spieltheoretischer Konzepte auch als Nash-Gleichgewicht eines wiederholt gespielten Spiels betrachtet werden können. ${ }^{98}$

Wichtig ist, dass Institutionen aus einer bestimmten Anzahl von Regeln bestehen und dass diese auf sich wiederholende soziale Verhaltensweisen einer bestimmten Gruppe von Individuen einwirken, die ein gemeinsames Ziel verfolgen. Für diesen Zweck haben Institutionen einen Durchsetzungsmechanismus, der mit Sanktionen bei Nichteinhaltung der Regeln verbunden ist. Die Aufrecht-

\footnotetext{
92 Schmoller (1900), S. 61.

93 Vgl. Schmoller (1900), S. 61.

94 Richter und Furubotn (2003), S. 7. Eine ähnliche Definition findet sich bei Nee und Ingram (1998), S. 19.

95 Vgl. Schotter (1986), S. 119; North (1990), S. 4 und 19; Nee und Ingram (1998), S. 19; Richter und Furubotn (2003), S. 7 f.

96 Vgl. Schotter (1986), S. 118.

97 Schotter (1981), S. 11.

98 Richter und Furubotn (2003), S. 9.
} 
erhaltung der Ordnung kontrolliert das individuelle Handeln, um einen Nutzen für andere Individuen zu erzeugen. ${ }^{99}$

Das Konzept der Institutionen ist auch auf Wirtschaftsförderprogramme anwendbar, dadurch dass sie aus einer Gesamtheit von Regeln zur Auswahl der geförderten Unternehmen und zur Durchführung der Fördermaßnahmen bestehen. Es ist davon auszugehen, dass diese Regeln, die von den die Förderprogramme beschließenden Politikern aufgestellt werden, das Verhalten der Bürokraten, die die Programme verwalten, und der Unternehmer, die Fördermittel beantragen und erhalten, beeinflussen. Der erste institutionelle Durchsetzungsmechanismus besteht darin, dass Unternehmer bzw. deren vorgeschlagene Projekte, welche die Auswahlkriterien nicht erfüllen, nicht gefördert werden. Ein zweiter Durchsetzungsmechanismus besteht darin, dass geförderte Unternehmer, die Fördermittel nicht zum vereinbarten Zweck einsetzen, die Mittel i. d. R. zurückzahlen müssen (vgl. Abschnitt 4.5.1.2).

Die Neue Institutionenökonomik basiert auf dem methodologischen Individualismus, welcher Institutionen unter Rückgriff auf das Verhalten der beteiligten Individuen definiert, die unterschiedliche Präferenzen und Ziele haben. ${ }^{100}$ Dieser Ansatz wird in Kapitel 4 konkret angewendet, wenn die Institution Innovationsförderung durch die verschiedenen an der Wirtschaftsförderung beteiligten Gruppen mit ihren speziellen Anreizen und Verhaltensweisen analysiert wird. Die dafür notwendigen Konzepte und Theorien werden in den folgenden Abschnitten hergeleitet. $\mathrm{Zu}$ den wichtigen Grundannahmen der Neuen Institutionenökonomik zählen neben dem methodologischen Individualismus die Berücksichtigung von unvollständiger Information (Abschnitt 3.1.1), wobei der Begriff und die Theorie der „bounded rationality“ von Simon $(1955,1959)$ besonders gewürdigt werden. Aus der unvollständigen Information ergeben sich die Existenz von Transaktionskosten (Abschnitt 3.1.2) und der Prinzipal-AgentAnsatz (Abschnitt 3.1.3).

\subsubsection{Unvollständige Information und „bounded rationality“}

In der klassischen Volkswirtschaftslehre gilt das Verhaltensmodell des Homo Oeconomicus. Danach verhalten sich Individuen vollkommen rational und eigennützig und maximieren ihren Nutzen, indem sie Grenznutzen und Grenzkosten einander gleich setzen. Allerdings wird dieses Verhaltensmodell von Simon (1955) wegen seiner rigiden und realitätsfernen Annahmen stark kritisiert: „, The task is to replace the global rationality of economic man with a kind of rational behavior that is compatible with the access to information and the computa-

99 Vgl. Ostrom (1986), S. 5; Commons (1934), S. 70 f.; Hodgson (1998), S. 179.

100 Vgl. Richter und Furubotn (2003), S. 3; Erlei et al. (1999), S. 319. 
tional capacities that are actually possessed by organisms, including man, in the kinds of environments in which such organisms exist. "101

Wenn ein Wirtschaftssubjekt eine Entscheidung trifft, ist es nicht in der Lage, alle bekannten Informationen aufzunehmen und in seine Entscheidungsfindung mit einzubeziehen. Dazu ist die Umgebung zu komplex und es existieren zu viele Informationen. Das Individuum versucht, sich auf die für seine Entscheidung relevanten Informationen zu konzentrieren. Dabei kann es nur einen Bruchteil der tatsächlich verfügbaren Informationen nutzen und interpretieren. ${ }^{102}$ Ein in der Literatur häufig verwendetes Beispiel ist der Schachspieler, der vor seinem Zug zwar vollkommene Information über alle regelkonformen Züge, die möglichen Antwortzüge seines Gegenspielers, seine eigenen Antworten darauf etc. hat. Der Spieler ist aber trotzdem nicht in der Lage, alle Informationen zu verarbeiten und den besten Zug zu bestimmen - selbst mit technischer Unterstützung nicht. So muss er sich auf einen Teil der verfügbaren Informationen beschränken und seine Entscheidung darauf stützen. ${ }^{103}$

Aufgrund der Komplexität der Umwelt ist es für jedes vor einer Entscheidung stehende Wirtschaftssubjekt außerdem zu kompliziert, ein Grenznutzenkalkül aufzustellen, um die optimale Handlungsstrategie und damit zusammenhängend das optimale Ergebnis zu errechnen. Da es für das Individuum unmöglich ist, alle Informationen zu erhalten und auszuwerten, beendet es die Suche nach und die Aufnahme von Informationen, sobald es auf ein akzeptables Ergebnis stößt. Diese Strategie wird auch als „satisficing“ bezeichnet. ${ }^{104}$ Die Erwartungen des Individuums, was ein akzeptables Ergebnis ausmacht, spielen hierbei eine sehr wichtige Rolle. Diese Erwartungen passen sich im Laufe der Zeit an, je nachdem ob das Individuum in der Vergangenheit in Hinblick auf potentiell erzielbare Ergebnisse enttäuscht oder erfreut wurde. ${ }^{105}$ Evtl. verändern sich im Laufe der Zeit auch durch die gemachten Erfahrungen und die gesammelten Informationen die Handlungsalternativen, die das Individuum für sich in Betracht zieht, um zu akzeptablen Ergebnissen zu gelangen. ${ }^{106}$ In Abgrenzung zur vollständigen Rationalität des Homo Oeconomicus spricht Simon (1955) von einer , limited rationality “107. Dieser Begriff wird später zur ,, bounded rationality “108.

Welche Informationen und Handlungsalternativen das Individuum wahrnimmt, hängt neben den eigenen Erfahrungen auch von den von anderen über-

101 Vgl. Simon (1955), S. 99.

102 Vgl. von Hayek (1945), S. 521 ff.; Simon (1959), S. 272 f.

103 Vgl. Richter und Furubotn (2003), S. 579.

104 Vgl. Simon (1979), S. 503; Simon (1955), S. 106 ff.; Simon (1959), S. 262 f.

105 Vgl. Simon (1955), S. 111; Simon (1959), S. 263.

106 Vgl. Simon (1955), S. $111 \mathrm{f}$.

107 Simon (1955), S. 113.

108 Simon (1979), S. 501. 
nommenen Verhaltensweisen ab. Die so genannten Kognitionen (,, Meinungen, Glaubensweisen, aber auch Wissenseinheiten "109) helfen bei zukünftiger Entscheidungsfindung unter Unsicherheit. Allerdings wird das Individuum auch von kognitiven Grenzen eingeengt, so dass es als Konsequenz gar nicht alle bestehenden Handlungsalternativen wahrnimmt. Hier können Institutionen eingreifen und die kognitiven Grenzen und somit auch das Verhalten der Individuen beeinflussen. ${ }^{110}$

Der Ansatz der „bounded rationality“ beinhaltet einen radikalen Bruch mit dem Modell des Homo Oeconomicus. Inwiefern dieser Ansatz mit der Neuen Institutionenökonomik vereinbar ist, welche das Modell des Homo Oeconomicus grundsätzlich beibehält, es aber um unvollständige Information, Transaktionskosten und Prinzipal-Agenten-Beziehungen (vgl. die späteren Abschnitte 3.1.2 und 3.1.3) erweitert, ist in der Literatur kontrovers diskutiert. Laut Simon selbst sind die beiden Theorien unvereinbar, weil das Modell der ,bounded rationality" die Annahme verwirft, dass Wirtschaftssubjekte ein dem Homo Oeconomicus irgendwie ähnlich rationales Kalkül aufstellen. Denn die individuellen Informationsanforderungen steigen $\mathrm{zu}$ sehr, wenn Unsicherheit und unvollständige Information mit Hilfe von Wahrscheinlichkeitsverteilungen in das Modell mit einbezogen werden. Denn wenn Individuen keine vollständige Information über das sie ursprünglich interessierende Ereignis haben, besitzen sie auch keinerlei Informationen über die Wahrscheinlichkeitsverteilung dieses Ereignisses. ${ }^{11}$ Gerade aus diesem Grund verwendet Williamson $(1990,1993)$ die „bounded rationality" aber als zentrale Verhaltensannahme für seine Transaktionskostenökonomik und integriert so die Theorie der „bounded rationality“ in die Neue Institutionenökonomik. ${ }^{112}$ Aufbauend auf Williamsons Argumentation wird in dieser Arbeit die „bounded rationality“ ebenfalls im Rahmen der Neuen Institutionenökonomik aufgeführt und verwendet.

\subsubsection{Der Transaktionskostenansatz}

Eine Transaktion stellt einen Austausch von Verfügungsrechten (,property rights") dar, wobei Verfügungsrechte wiederum , die mit materiellen und immateriellen Gütern verbundenen, institutionell legitimierten Handlungsrechte eines oder mehrerer Wirtschaftssubjekte "113 sind.

109 Bizer (1998), S. 12.

110 Vgl. Bizer (1998), S. 12 ff.

111 Vgl. Simon (2000), der auch die Spieltheorie und die Theorie der rationalen Erwartungen mit einer ähnlichen Argumentation kritisiert.

112 Vgl. Williamson (1990), S. 51; Williamson (1993), S. 11.

113 Picot und Dietl (1990), S. 178. 
Transaktionskosten werden definiert als Kosten, die damit zusammenhängen, dass Verfügungsrechte bestimmt, getauscht und durchgesetzt werden. ${ }^{114}$ Das Transaktionsobjekt stellen also die ,property rights" dar und nicht die weitergegebenen Güter und Dienstleistungen. ${ }^{1{ }^{5}}$ Allgemein ausgedrückt entsprechen Transaktionskosten den festen und variablen Kosten des wirtschaftlichen Handelns und sind der eigentliche Grund für die Entstehung von Institutionen, da sich die beteiligten Individuen nicht sofort kostenlos an neue Gegebenheiten anpassen können. ${ }^{16}$

Coase (1937) erkannte als erster, dass die Benutzung von Märkten mit Kosten verbunden ist, weswegen der Koordinationsmechanismus des Unternehmens dem Markt vorzuziehen sein kann: „The main reason why it is profitable to establish a firm would seem to be that there is a cost of using the price mechanism. "117 Die Begründung für die Existenz von Unternehmen sind somit die Kosten der Benutzung des Marktmechanismus bzw. die Transaktionskosten. Zu diesen Kosten zählen laut Coase Kosten der Information über die auf dem Markt vorherrschenden Preise sowie Verhandlungskosten und Kosten der Vertragsschließung. ${ }^{118}$

Doch nicht nur die Durchführung von Transaktionen auf dem Markt ist mit Kosten verbunden. Auch bei der Durchführung von Transaktionen innerhalb eines Unternehmens fallen bestimmte Kosten an, die Coase in seine Analyse mit einbezieht. Die Transaktionskosten innerhalb des Unternehmens steigen mit der Unternehmensgröße an, außerdem setze der Unternehmer mit steigender Anzahl der zu organisierenden Transaktionen die Produktionsfaktoren immer weniger effizient ein. Deswegen gebe es keine Gefahr, dass eine Volkswirtschaft irgendwann nur noch aus einem Unternehmen bestehe, unter dessen Dach alle Transaktionen abgewickelt werden. Ein Unternehmen werde nur so lange wachsen, bis die Grenzkosten der Produktion einer Transaktion innerhalb des Unternehmens identisch seien mit den Grenzkosten der Durchführung der Transaktion auf dem Markt. ${ }^{119}$

Als grundlegende Voraussetzung für die Existenz von Transaktionskosten dient neben dem im vorhergehenden Abschnitt erläuterten Konstrukt der ,bounded rationality“ der Opportunismus der Wirtschaftsakteure. Dabei ist Opportunismus als Verschärfung der Annahme des methodologischen Individualismus zu verstehen. Wirtschaftssubjekte verfolgen ihre Eigeninteressen, und dabei ist

114 Vgl. Picot und Dietl (1990), S. 178.

$115 \mathrm{Vgl}$. für eine ausführliche Diskussion des Transaktionskostenansatzes Haverkamp (2007), S. $103 \mathrm{ff}$.

116 Vgl. Williamson (1990), S. 1 f.; Richter und Furubotn (2003), S. 585 und 592.

117 Coase (1937), S. 390.

118 Vgl. Coase (1937), S. $390 \mathrm{f}$.

119 Vgl. Coase (1937), S. 394 f. 
ihnen jedes Mittel recht, auch List, Lüge und Betrug. ${ }^{120}$ Deswegen bestehen bei unvollständigen Verträgen verschiedene Gefahren, welche im Rahmen der Prinzipal-Agent-Beziehung im folgenden Abschnitt näher erläutert werden.

\subsubsection{Informationsasymmetrien und Prinzipal-Agent-Beziehungen}

Der Prinzipal-Agent-Ansatz beschreibt eine Beziehung zwischen mindestens zwei Individuen, von denen einer Auftraggeber (Prinzipal) und der andere dessen Vertreter (Agent) ist. Die Entscheidungen und das Handeln des Agenten beeinflussen nicht nur seinen Nutzen, sondern auch denjenigen des Prinzipals. ${ }^{121}$ Es bestehen Interessenkonflikte zwischen dem Prinzipal, der an einem guten Auftragsergebnis interessiert ist, und dem Agenten, der seinen eigenen Nutzen maximieren will, welcher neben dem Auftragsergebnis auch von seinen Handlungsentscheidungen und seinem mit Opportunitätskosten verbundenen Einsatz beeinflusst wird. ${ }^{122}$

Während in der klassischen Volkswirtschaftslehre jederzeit vollkommene Information herrscht, ist die Information in einer Prinzipal-Agent-Beziehung asymmetrisch verteilt, so dass eine kostenlose Überwachung des Agenten durch den Prinzipal nicht möglich ist. Bei diesen asymmetrisch verteilten Informationen handelt es sich entweder um "hidden information" oder "hidden action“. Der erste Begriff bedeutet, dass der Agent über private Informationen oder Beobachtungen verfügt, die der Prinzipal nicht besitzt. Der Begriff ,hidden action“ bezeichnet das Phänomen, dass der Prinzipal den Einsatz bzw. die unternommenen Handlungen des Agenten nicht beobachten kann. In diesem Zusammenhang tritt „moral hazard“" auf: das moralische Risiko, das nach Vertragsschluss auftritt, wenn der Agent zum „ex post“-Opportunismus neigt. ${ }^{123}$

Im Fall einer oben beschriebenen Prinzipal-Agent-Beziehung besteht eine Lösungsmöglichkeit für die Interessenkonflikte zwischen Prinzipal und Agent in einer bestimmten Vertragsausgestaltung zwischen den beiden Akteuren: Der Agent erhält eine Entlohnung in Höhe des Auftragsergebnisses, die sich um einen festen Betrag verringert, den der Prinzipal bekommt. ${ }^{124}$ Bei Einführung von Risiko bzw. Unsicherheit als einem Bestimmungsfaktor des Auftragsergebnisses neben dem Einsatz des Agenten ist diese Lösung allerdings nicht optimal. Wenn der Prinzipal risikoneutral und der Agent risikoavers ist, wird eine Vertragsstruktur vorteilhaft, in der der Prinzipal einen Teil des Risikos übernimmt. Da nun der Agent nur noch anteilig an einer Erhöhung des Auftragsergebnisses

120 Vgl. Williamson (1990), S. 53 f.; Williamson (1993), S. 11 f.

121 Vgl. Jensen und Meckling (1976), S. 308; Sappington (1991), S. 46 f.; Arrow (1985), S. 37; Pratt und Zeckhauser (1985), S. 2.

122 Vgl. Sappington (1991), S. 47; Arrow (1985), S. 43 f.

123 Vgl. Arrow (1985), S. 38 ff.; Richter und Furubotn (2003), S. 584; Voigt (2002), S. 104.

124 Vgl. Sappington (1991), S. 47. 
durch seinen Arbeitseinsatz profitiert, wendet er weniger Einsatz auf, als die für den Prinzipal optimale Menge beträgt. ${ }^{12}$

Lösungsmöglichkeiten für diese Prinzipal-Agent-Probleme bestehen erstens in der Auswahl der Agenten mit Hilfe des ,signalling“, d.h. der Interpretation der von den Agenten ausgesendeten Signale durch den Prinzipal. Zweitens kann der Prinzipal mit Hilfe eines guten Beobachtungssystems (,monitoring“) versuchen, direkt oder indirekt Informationen über den Einsatz seines Agenten zu erhalten. Drittens besteht die Möglichkeit eines vom Prinzipal initiierten Wettbewerbs zwischen den Agenten, in dem derjenige Agent mit dem besten Auftragsergebnis belohnt und/oder derjenige Agent mit dem schlechtesten Ergebnis bestraft wird. ${ }^{126}$

Zusammenfassend beschreibt die Prinzipal-Agent-Theorie also die Gefahr, dass das Handeln des Agenten vom vertraglich vereinbarten Handeln abweicht. Dagegen vorgehende Kontrollen und Sanktionen sind nicht kostenlos wie in der klassischen Volkswirtschaftslehre, sondern mit Transaktionskosten (vgl. Abschnitt 3.1.2) verbunden. Eine klassische Prinzipal-Agent-Beziehung besteht zwischen Politikern und Bürokraten dadurch, dass die Politiker in der Legislative und Exekutive Gesetze und Richtlinien beschließen, die von den Bürokraten mit entworfen und anschließend im Detail umgesetzt werden. Bezogen auf die Thematik der Innovationsförderung besteht auch zwischen Bürokraten und Unternehmern eine Prinzipal-Agenten-Beziehung. Diese Prinzipal-Agent-Beziehungen werden in Kapitel 4 untersucht.

Die in den letzten Abschnitten beschriebenen Konzepte der Neuen Institutionenökonomik gelten für alle Wirtschaftssubjekte und somit gleichermaßen für Politiker, Bürokraten und Unternehmer. Es gibt aber auch spezielle Theorien der Neuen Institutionenökonomik für die beiden beteiligten Akteursgruppen der Politiker und Bürokraten, die ihre jeweiligen Anreize und Interessen gesondert untersuchen. Diese Theorien werden zunächst in den Abschnitten 3.2 und 3.3 vorgestellt, um dann in Kapitel 4 alle vorgestellten Theorieansätze der Neuen Institutionenökonomik zusammen auf die Thematik der Innovationsförderung anzuwenden.

125 Vgl. Sappington (1991), S. 49 ff.; Arrow (1985), S. 44 f. Auch für den Fall, dass der Agent nicht an den Vertrag gebunden ist oder vor Abschluss des Vertrags nähere Informationen über das das Auftragsergebnis beeinflussende Risiko hat, kommt es dazu, dass der Agent einen geringeren Einsatz leistet, vgl. Sappington (1991), S. 50 f.

126 Vgl. Sappington (1991), S. 52 ff.; Arrow (1985), S. 45 ff.; Pratt und Zeckhauser (1985), S. $5 \mathrm{f}$. 


\subsection{Politiker in der Neuen Politischen Ökonomie}

Ein Politiker wird allgemein definiert als „Person, die haupt-oder nebenamtlich an der politischen Willensbildung und dem politischen Entscheidungsprozess beteiligt und hierfür rechenschaftspflichtig ist " ${ }^{127}$ Die Theorierichtung der Neuen Politischen Ökonomie setzt sich speziell mit den Interessen und dem Verhalten von Politikern auseinander, die separat von den in Abschnitt $3.3 \mathrm{zu}$ behandelnden Bürokraten analysiert werden. Theorieansätze wie das LeviathanModell von Brennan und Buchanan (1980), folgen dieser Argumentation nicht und modellieren den Staat als Ganzes, indem Politik- und Bürokratieebene gemeinsam als nahezu allmächtige Institution dargestellt werden, die nur durch verfassungsrechtliche Regelungen im Zaum gehalten werden kann. ${ }^{128}$ Derartige Theorieansätze werden im Rahmen dieser Arbeit nicht behandelt. Stattdessen werden im Folgenden die Theorieansätze der Neuen Politischen Ökonomie und dabei insbesondere das Modell der politischen Konjunkturzyklen (Abschnitt 3.2.1) und ,rent seeking“-Ansätze (Abschnitt 3.2.2) erläutert.

Die Neue Politische Ökonomie sieht Politiker nicht als wohlwollende soziale Planer, sondern bezieht den individuellen Nutzen der Politiker in die Analyse mit ein. Der individuelle Nutzen wird von Faktoren wie Einkommen, Prestige und Macht bestimmt. Um diese mit dem politischen Amt verknüpften Faktoren auch in Zukunft zu behalten, ist der Politiker an seiner Wiederwahl interessiert. ${ }^{129} \mathrm{Um}$ seine Wiederwahl zu erlangen, ist der Politiker seinen Wählern verpflichtet und sollte seine Wahlversprechen erfüllen. Politiker gehören aber auch bestimmten Parteien an, in denen sich Individuen mit ähnlichen Interessen und Zielen zusammengeschlossen haben. An der Erfüllung dieser Ziele ist der Politiker interessiert, wenn er in eine politische Position gewählt wurde. ${ }^{130}$ Abgesehen von der eigenen politischen Überzeugung darf sich der Politiker auch deswegen nicht zu weit von den Zielen seiner Partei entfernen, weil ihm diese ansonsten den Rückhalt verweigert. Dann wird es schwierig für den Politiker, seine politischen Projekte durchzusetzen und zur Wiederwahl aufgestellt zu werden.

Der Politiker muss also versuchen, drei Arten von Zielen gleichzeitig gerecht zu werden: denen seiner Partei, denen seiner Wähler und nicht zuletzt seinen eigenen. Der folgende Abschnitt beschäftigt sich mit den ersten beiden Zielen, indem der Zusammenhang zwischen wirtschaftlicher Entwicklung, Parteipräferenzen und Wiederwahl eines amtierenden Politikers mit Hilfe des Modells der politischen Konjunkturzyklen modelliert wird. In Abschnitt 3.2.2 geht es

127 Schmidt (1995), S. 731.

128 Vgl. Brennan und Buchanan (1980), S. $15 \mathrm{ff}$.

129 Vgl. Frey und Schneider (1978), S. 178.

130 Vgl. Hartig (1990), S. 104 f. 
daran anschließend um die Verwirklichung der persönlichen Ziele des Politikers, die mit Hilfe der Theorie des ,rent seeking“ beschrieben werden.

\subsubsection{Politische Konjunkturzyklen}

Das Modell der politischen Konjunkturzyklen basiert auf Nordhaus (1975) und beschreibt eine Situation, in der die amtierende Regierung die Wirtschaftspolitik benutzt, um im Wahljahr einen ökonomischen Aufschwung zu erzeugen. Die Bürger bewerten die aktuelle wirtschaftliche Entwicklung im Wahljahr und vergleichen diese mit einem Referenzwert, der beispielsweise aus den Erfahrungen der Vergangenheit gebildet wird. Die Chancen der amtierenden Regierung auf Wiederwahl steigen mit einer positiven wirtschaftlichen Entwicklung im Wahljahr. ${ }^{131}$ Entscheidend für dieses Modell ist die Annahme, dass die Wähler keinen Anreiz haben, hohe Transaktionskosten auf sich zu nehmen, um sich vollständig über die Regierungsarbeit zu informieren. Deshalb berücksichtigen sie nur die wichtigsten ökonomischen Größen, die ihr wirtschaftliches Handeln direkt beeinflussen. Die Bürger gehen davon aus, dass die Wirtschaftsentwicklung vollständig in der Macht der Regierung liegt und ignorieren alle alternativen Einflussgrößen. ${ }^{132}$ Wichtig ist die Modellannahme, dass die Wähler in dem Sinne kurzsichtig sind, dass sie in ihrer Wahlentscheidung nicht die wirtschaftliche Entwicklung der gesamten Regierungszeit berücksichtigen, sondern sich auf die Gegenwart und die gerade zurückliegende Vergangenheit konzentrieren. Für diese Annahme gibt es vielfältige empirische Belege. ${ }^{\text {. }} 3$

Im klassischen Modell des Konjunkturzyklus sind Arbeitslosigkeit und Inflation die beiden entscheidenden wirtschaftlichen Variablen, die die Wähler interessieren. Dabei wird die Arbeitslosigkeit laut Nordhaus von den Wählern am wichtigsten bewertet. Deswegen halten Politiker die Arbeitslosigkeit im Wahljahr möglichst niedrig und nehmen dafür eine höhere Inflation in Kauf. Nach der Wahl bekämpfen die Politiker die hohe Inflationsrate, indem sie eine Erhöhung der Arbeitslosenquote in Kauf nehmen. Dieses Modell geht somit zum einen vom Vorhandensein des durch die Phillips-Kurve beschriebenen negativen Zusammenhangs zwischen Inflation und Arbeitslosigkeit aus und schreibt zum anderen den Politikern die Macht zu, sich eine Kombination der beiden Variablen auszusuchen. ${ }^{134}$

Im Grundmodell haben Politiker keinerlei Präferenzen hinsichtlich der Ausprägung der Variablen Inflation und Arbeitslosigkeit. In Erweiterungen der Konjunkturzyklusmodelle mit Hilfe von so genannten Partisan-Ansätzen werden jedoch Parteipräferenzen bezüglich der interessierenden Wirtschaftsvariablen

131 Vgl. Nordhaus (1975), S. 172 f.; Paldam (1997), S. 346.

132 Vgl. Frey und Schneider (1978), S. 175; Nordhaus (1975), S. 172 f.

133 Vgl. Paldam (1997), S. 346.

134 Vgl. Nordhaus (1975), S. 170 und 184. 
explizit berücksichtigt. Besteht die amtierende Regierung aus Politikern der linksgerichteten Parteien, so legen diese ebenso wie ihre Wähler einen hohen Wert auf eine niedrige Arbeitslosigkeit zum Wahlzeitpunkt. Dagegen bevorzugen rechtsgerichtete Parteien und ihre Wähler eine niedrige Inflationsrate und versuchen deswegen zum Wahlzeitpunkt eine möglichst niedrige Inflationsrate zu erreichen. ${ }^{135}$

Wie oben erwähnt, sind im klassischen Modell des Konjunkturzyklus Arbeitslosigkeit und Inflation die beiden entscheidenden Variablen, die die Wähler interessieren. Allerdings weist schon Nordhaus auf die Möglichkeit anderer Variablen, z. B. öffentliche Investitionen, hin. ${ }^{136}$ Später fügen Frey und Schneider (1978) noch das Konsumwachstum als relevante Einflussgröße hinzu. ${ }^{137}$ Bei Verwendung des Wirtschaftswachstums als die den Wähler interessierende Variable lässt sich ein Zusammenhang zwischen dem Modell der politischen Konjunkturzyklen und Innovationsförderung herstellen, wie in Abschnitt 4.3.2 näher ausgeführt wird. Innovationsförderung kann als politisches Instrument eingesetzt werden, um das regionale Wirtschaftswachstum zu erhöhen und so die Wahlchancen der politischen Amtsinhaber zu steigern.

\subsection{2 „Rent seeking“ und „lobbying“}

Während sich der vorige Abschnitt mit der Berücksichtigung der Ziele von Wählern und Parteien durch die Politiker beschäftigte, behandelt die „rent seeking“-Theorie die persönlichen Interessen der Politiker. Der Begriff des „rent seeking" beschreibt eine Situation, in der Politiker Interessengruppen die Errichtung oder Verteidigung einer Monopolposition ermöglichen, wodurch diese von Monopolrenten profitieren, während die Konsumenten der entsprechenden Güter und Dienstleistungen durch den Monopolpreis eine Verringerung ihrer Rente in Kauf nehmen müssen. Insgesamt kommt es zu einem gesamtgesellschaftlichen Wohlfahrtsverlust. ${ }^{138}$

Buchanan (1980) differenziert zwischen drei Ausgabenarten, die sich zu einem durch das ,rent seeking“ anfallenden gesellschaftlichen Wohlfahrtsverlust addieren. Erstens sind die Anstrengungen und Ausgaben der potentiellen Profiteure des Monopols zu nennen, die z. B. im Rahmen des „lobbying“ entstehen. Zweitens müssen die Anstrengungen der Regierungsangehörigen berücksichtigt

135 Für einen Überblick über Partisan-Ansätze und politische Konjunkturzyklen vgl. Paldam (1997), S. 356 ff. Frey und Schneider (1978) stellen ein politökonomisches Modell auf, das zum einen aus einer Popularitätsfunktion der Wähler für die Regierung und zum anderen der politischen Reaktionsfunktion zur Sicherung der Wiederwahl besteht, und welches die Autoren mit positiven Resultaten für den Zeitraum 1953-1975 für die USA empirisch überprüft haben.

136 Vgl. Nordhaus (1975), S. 169 und 188.

137 Vgl. Frey und Schneider (1978), S. 174.

138 Vgl. Buchanan (1980), S. 7 f.; Tollison (1982), S. 575 f. 
werden, die Mittel von den Interessengruppen erhalten und auf deren Anliegen reagieren. Unter die Anstrengungen der Regierungsangehörigen fallen auch die Anstrengungen zur Erlangung einer bestimmten Machtposition, um in dieser Position den Interessengruppen bei Errichtung oder Verteidigung ihrer Monopolposition zu helfen und sich dadurch eigene Vorteile zu verschaffen. Ein Beispiel für diese Anstrengungen ist die mit Kosten verbundene Humankapitalerhöhung der Politiker, um sich für eine Machtposition zu qualifizieren. Drittens dürfen die Beeinflussung der Wohlfahrt dritter Gruppen durch die ,rent seeking“-Aktivitäten nicht vergessen werden. ${ }^{139}$ Dabei gehören Bestechungsgelder selbst nicht zwingend zum gesellschaftlichen Wohlfahrtsverlust, sondern stellen eine Form der Redistribution zwischen verschiedenen gesellschaftlichen Gruppen dar. Der soziale Wohlfahrtsverlust entsteht durch die Transaktionskosten, die mit den „rent seeking“-Aktivitäten bei allen beteiligten Gruppen verbunden sind. Es gibt in der Literatur eine rege Diskussion über die Höhe des sozialen Wohlfahrtsverlustes und ob er die Monopolrente möglicherweise vollständig verschlingt. $^{140}$

Die Theorie des „rent seeking“ betrifft vielfältige Politikfelder, beispielsweise staatlich vergebene Monopole, Außenhandelspolitik oder auch direkte Unternehmenstransferzahlungen, zu denen auch Subventionen im Innovationsförderbereich gehören. ${ }^{141}$ Empirische Schätzungen des Wohlfahrtsverlustes durch „rent seeking“ in einer Volkswirtschaft ergeben je nach Schätzungsmethode teilweise erhebliche Summen, die in der Vergangenheit bis zu $50 \%$ des BIP ausmachten. ${ }^{142}$ Dieser Wohlfahrtsverlust kam zwar überwiegend durch Handelshemmnisse zustande, doch können auch Subventionen hier eine Rolle spielen. Entscheidend für ,rent seeking“ sind die Anstrengungen der Interessengruppen, die die Politikträger in Form von Geldzahlungen, wie z. B. Parteispenden, oder der Lieferung von Informationen im Zuge des Wahlkampfes oder der Gesetzgebung (,lobbying“) zu beeinflussen versuchen. ${ }^{143}$ So könnten auch die Unternehmer vertretenden Interessengruppen versuchen, die Politik zu einer Ausweitung der Innovationsförderung zu drängen, weil einzelne Interessengrup-

139 Vgl. Buchanan (1980), S. $12 \mathrm{ff}$.

140 Vgl. Krueger (1974), S. 292 f.; Tollison (1997), S. 508 ff.; Mueller (2003), S. 335 ff.

141 Vgl. Mueller (2003), S. $343 \mathrm{ff}$.

142 Für einen Überblick über empirische Studien zum sozialen Wohlfahrtsverlust durch „rent seeking“ vgl. Tollison (1997), S. 512 ff. Die erste empirische Schätzung findet sich bei Krueger (1974), die sich auf den Wohlfahrtsverlust durch ,rent seeking“ nur im Bereich von Importlizensen für die Türkei (15\% des BIP im Jahr 1968) und Indien ( $7 \%$ des BIP im Jahr 1964) konzentriert.

143 Es gibt einen eigenen Theoriestrang der Neuen Politischen Ökonomie, der sich mit dem Bereich der Interessengruppen und ihren Anstrengungen, die Politik zu beeinflussen, beschäftigt. Die einflussreichste Formalisierung stammt von Becker (1983) und zeigt die vielfältige Anwendbarkeit des Interessengruppenansatzes auf die Politik. Ein guter Überblick über die gesamte Theorierichtung findet sich bei Austen-Smith (1997). 
penmitglieder von der Förderung profitieren. Diese Argumentation wird in Abschnitt 4.3.2 ausgeführt.

\subsubsection{Fazit}

Die Neue Politische Ökonomie verlässt das in weiten Teilen der Volkswirtschaftslehre noch vorherrschende Bild des wohlwollenden sozialen Planers und berücksichtigt stattdessen den individuellen Nutzen der Politiker, der von Einkommen, Prestige und Macht beeinflusst wird. Diese Faktoren sind mit seinem politischen Amt und somit seiner Wiederwahl eng verknüpft. Deswegen muss der Politiker neben seinen eigenen Zielen die Ziele seiner Wähler und der ihn für ein Amt aufstellenden Partei beachten. Das Modell der politischen Konjunkturzyklen stellt die Beeinflussung der Wiederwahl durch Manipulation der wirtschaftlichen Entwicklung durch den Amtsinhaber dar. Im Grundmodell von Nordhaus werden Arbeitslosigkeit und Inflation als die die Wähler interessierenden wirtschaftlichen Variablen verwendet, doch ist auch das durch die Innovationstätigkeit beeinflusste Wirtschaftswachstum eine sinnvoll anzuwendende Größe. Der mögliche Einfluss der Interessengruppen auf die Politik wurde im Rahmen der „rent seeking“-Theorie und des „lobbying“ vorgestellt. Dabei gewähren Politiker Interessengruppen eine irgendwie geartete Monopolposition und die dazugehörige Monopolrente. Die Anwendung der Theorien auf die Thematik der Innovationsförderung erfolgt im Rahmen der institutionenökonomischen Analyse des NIFP in Kapitel 4.

\subsection{Bürokraten als Verwalter der Wirtschaftsfördermittel}

Die Bürokratie ist dafür verantwortlich, die von den Politikern entworfenen Innovationsförderprogramme umzusetzen und die Fördermittel zu vergeben. Um das Verhalten und die Anreize der Bürokraten zu analysieren, gibt es eine eigene Theorierichtung innerhalb der Neuen Institutionenökonomik: die Theorie der Bürokratie, die im Folgenden vorgestellt wird. Der Schwerpunkt der folgenden Abschnitte liegt auf dem Bürokratie-Modell von Niskanen (1994) und seinen Einschränkungen bzw. Erweiterungen, weil es bis heute der einflussreichste und am stärksten diskutierte Ansatz ist. ${ }^{144}$ Zunächst wird im folgenden Abschnitt das Grundmodell des Budgetmaximierers von Niskanen vorgestellt. Daran anschließend werden Einschränkungen der Anwendbarkeit des Niskanen-Modells (Abschnitt 3.3.2), alternative Theorieansätze (Abschnitt 3.3.3) und die empirische Evidenz (Abschnitt 3.3.4) diskutiert.

144 Vgl. Moe (1997), S. 458. 


\subsubsection{Die Bürokratie als Budgetmaximierer nach Niskanen}

Die kommunale Verwaltung ist eine Bürokratie im Sinne von Niskanen, also eine nicht auf Gewinn ausgerichtete Organisation, deren Einkommen nicht (nur) über den Verkauf ihres Outputs, sondern auch über wiederkehrende externe Finanzmittel erfolgt. ${ }^{145}$ Ihre Mitglieder, die Bürokraten, sind weder in ihrer Ernennung noch in ihrer Arbeit von der Meinung der Wähler abhängig. Obwohl die Entscheidungsgewalt der Bürokraten nicht die Erstellung der Regeln zur Vergabe von Wirtschaftsförderung, also den Beschluss der Förderprogramme, beinhaltet, ist ihre Macht ein nicht zu vernachlässigender Faktor. Mueller (2003) definiert den Begriff Macht allgemein als ,ability or capacity to do something "146. Muellers Definition basiert auf der Definition des Philosophen Russell (1947), welcher Macht als "Hervorbringen beabsichtigter Wirkungen "147 beschreibt und Macht über Individuen in drei Formen aufgliedert: erstens direkte physische Gewalt über den Körper, zweitens die Verwendung von Belohnungen und Strafen als Anreize, drittens Beeinflussung der Meinung durch Propaganda, also Informationen. ${ }^{148}$

Die Macht der Bürokraten basiert im Wesentlichen auf der dritten Form von Macht, den wertvollen Informationen, die die Bürokraten zu Experten mit Informationsvorteilen gegenüber anderen Akteuren machen. Es herrscht eine Prinzipal-Agent-Beziehung zwischen Politikern und Bürokraten vor, die von einer Informationsasymmetrie entscheidend beeinflusst wird. Crozier (1964) illustriert diese Macht der Information anhand des Beispiels eines Großunternehmens, in dessen Produktionsabläufen es nur eine Unsicherheit gibt: Von Zeit zu Zeit brechen die Maschinen zusammen und können nur von den Mechanikern repariert werden. Diese Information über den Maschinenmechanismus gibt den Mechanikern Macht - und zwar nicht nur über die Arbeiter, die einen vertraglich vereinbarten Output pro Tag fertig stellen müssen und deswegen auf eine schnelle Reparatur angewiesen sind, sondern auch über die Aufseher, die in der Firmenhierarchie eigentlich über den Mechanikern stehen, aber deren Expertenwissen nichts entgegenzusetzen haben. ${ }^{149}$ Angewendet auf das hier zu untersuchende Thema stellen die Mechaniker die Bürokraten dar, die im inhaltlichen Bereich der Durchführung der Innovationsförderprogramme Expertenwissen besitzen, während die Politiker als die hierarchisch über ihnen stehenden „Aufseher" zwar Weisungsbefugnis haben, aber gleichzeitig auf das Wissen und die inhaltliche Unterstützung durch die Bürokraten angewiesen sind, um eine erfolg-

145 Vgl. Niskanen (1994), S. 15.

146 Mueller (2003), S. 360.

147 Russell (1947), S. 29

148 Vgl. Russell (1947), S. 29.

149 Vgl. Crozier (1964), S. 61-142, vgl. zur Interpretation dieses Beispiels auch Mueller (2003), S. 361. 
reiche Wirtschaftspolitik zu betreiben. Diese stellt ihren durch die Wähler bewerteten Output dar, der wiederum von den Wählern beurteilt wird. Es herrscht eine Prinzipal-Agent-Beziehung zwischen den die Förderprogramme entwerfenden und beschließenden Politikern als Prinzipalen und den für die Durchführung der Programme verantwortlichen Bürokraten als Agenten. Diese PrinzipalAgent-Beziehung und die dazugehörige Informationsasymmetrie zwischen Politikern und Bürokraten werden ausführlicher in Abschnitt 4.4.2 untersucht.

Der bürokratische Handlungsspielraum ist jedoch nicht nur ein Resultat der herrschenden Informationsasymmetrie. Politiker sorgen teilweise auch bewusst für einen relativ großen bürokratischen Handlungsspielraum, indem sie Gesetze und Richtlinien vage formulieren, weil vor Inkraftreten der Regelung nicht alle möglichen Auswirkungen auf verschiedene Akteursgruppen bedacht werden können. Der Handlungsspielraum der Bürokraten kann dann einer verbesserten Implementierung im Rahmen des Gesetzgebers dienen. ${ }^{150}$ Nach Niskanen besitzen die Bürokraten neben ihrem Informationsmonopol noch eine zweite Form von Macht, die so genannte ,agenda power“, dadurch dass sie den Politikern ein Angebot für die Produktion eines bestimmten Outputs mit Hilfe eines bestimmten Budgets unterbreiten können, was als „take-it-or-leave-it offer“ bezeichnet wird. ${ }^{15}$

Neben dem Informationsvorteil und der ,agenda power“ gibt es noch einen weiteren Grund für eine besondere Stellung der Bürokratie, nämlich die Beschaffenheit des Bürokratieoutputs, welcher nicht marktgängig, komplex und multidimensional ist. Somit ist die Messung des Outputs der Bürokraten und damit zusammenhängend die Überwachung ihrer Effizienz sehr schwierig. ${ }^{152}$ Es ist folglich problematisch für Politiker, die Bürokratie zu überwachen. Bürokraten können als Konsequenz hierauf ihre eigenen Ziele neben den offiziellen Zielen der Bürokratie verfolgen. Nun ist es Bürokraten nicht möglich, Gewinnziele $\mathrm{zu}$ verfolgen und sich über Gewinnbeteiligungen höhere Einkommen zu verschaffen, wie es in der freien Wirtschaft üblich ist. Deswegen richtet sich ihr Streben statt nach höheren Löhnen hauptsächlich nach nichtmonetären Vorteilen, wie gut ausgestatteten Büroräumen, einer hohen Anzahl an Untergebenen oder Ansehen sowie Bequemlichkeit der Arbeit, in dem Sinne dass die Arbeit mit möglichst wenig Stress und Anstrengung verbunden ist. Insgesamt werden diese Ziele in der Literatur unter dem Begriff X-Ineffizienz ${ }^{153}$ zusammengefasst. ${ }^{154}$ Bis auf die Bequemlichkeit der Arbeit hängen alle diese Vorteile direkt

150 Vgl. Mueller (2003), S. 389. Zur optimalen Aufgabenteilung zwischen Politikern und Bürokraten vgl. Alesina und Tabellini (2007).

151 Vgl. Moe (1997), S. 458 f.; Mueller (2003), S. 365.

152 Vgl. Mueller (2003), S. 363. Zum Problem der Leistungsmessung in Politik und Verwaltung vgl. Bogumil (2004).

153 Dieser Begriff tauchte als erstes bei Leibenstein (1966), S. 392, auf.

154 Vgl. Mueller (2003), S. 362 f. und S. 368. 
mit der Größe und dem Budget der jeweiligen Abteilung zusammen. Je größer und komplexer die Abteilung ist, desto schwieriger ist die externe Überwachung, so dass auch die Bequemlichkeit der Arbeit positiv mit der Abteilungsgröße zusammenhängt. Aus diesen Gründen haben Bürokraten einen Anreiz, ihr Budget zu maximieren und zu diesem Zweck so viele zusätzliche Aufgaben wie möglich anzuhäufen. ${ }^{155} \mathrm{Da}$ diese Argumentation intuitiv einleuchtet, verwendet der Großteil der Literatur zur Bürokratietheorie das Modell des budgetmaximierenden Bürokraten. Dieses Modell ist in der Realität allerdings nur eingeschränkt gültig, wie im folgenden Abschnitt ausgeführt wird.

\subsubsection{Einschränkungen der Macht der Bürokraten}

In der Realität ist aus fünf Gründen davon auszugehen, dass die Bürokratie in ihrer Macht und Entscheidungsgewalt eingeschränkt ist. Erstens ist die Entscheidung über den zu produzierenden Output und das Budget meist nicht vollständig der Bürokratie überlassen. Es ist davon auszugehen, dass die zuständigen Politiker konkrete Outputvorgaben machen und nicht jedes von den Bürokraten vorgeschlagene Budget akzeptieren. Auch ist unklar, warum die Bürokratie die Nachfragefunktion der Politik und damit zusammenhängend ihre Akzeptanzschwellen für die Annahme bestimmter Kombinationen aus Output und Budget wie im Falle eines vollkommenen Monopols kennen sollte. Stattdessen steht die Politik als Monopsonist dem Monopol der Bürokratie gegenüber und hat ebenfalls eine gewisse Machtstellung inne. Die Annahme der ,agenda power" der Bürokraten ist somit unrealistisch. Es bleibt die Informationsasymmetrie als wesentliche Machtbasis der Bürokratie. ${ }^{156}$

Zweitens besteht die Möglichkeit, dass die Politik ihre Monopolstellung als Finanzier der Bürokratie erkennt und verhandlungstaktisch ausnutzt, beispielsweise in Form von Drohungen einer zukünftigen drastischen Mittelkürzung für einen bestimmten Bürokratiebereich. ${ }^{157}$

Drittens ergibt sich eine Machteinschränkung bei Aufweichung der Monopolannahme für die Bürokratie, indem ein möglicher Wettbewerb zwischen verschiedenen öffentlichen Einrichtungen um die Ausführung bestimmter Aufträge berücksichtigt wird. ${ }^{158}$

Viertens nehmen Institutionen, wie z. B. Rechnungshöfe, die die Mittelverwendung der Bürokratien überwachen, eine wesentliche Rolle bei der Einschränkung der Entscheidungsmacht der Bürokraten ein. Des Weiteren besteht die Möglichkeit, dass einzelne Bürokraten Einzelheiten die Arbeitsweise und

155 Vgl. Mueller (2003), S. 362 ff. und S. 368; Vaubel (1991), S. 36; Duncombe et al. (1997), S. 5.

156 Vgl. Moe (1997), S. 459 f.

157 Vgl. Mueller (2003), S. 367 f.; Duncombe et al. (1997), S. 5; Wintrobe (1997), S. 431.

158 Vgl. Mueller (2003), S. 367 f.; Duncombe et al. (1997), S. 5; Wintrobe (1997), S. 431. 
Vorgänge innerhalb ihrer Abteilung betreffend nach außen tragen (so genannte „whistle-blowers"). Im Fall einer Entdeckung von exzessiver Mittelverschwendung besteht die Gefahr, dass die Abteilung bestraft wird, z. B. in Form eines geringeren zukünftigen Budgets oder Personalreduzierungen. ${ }^{159}$ In diesem Fall ist die Erhöhung des Budgets über das unbedingt notwendige Maß hinaus mit zwei gegenläufigen Einflüssen auf den Nutzen des Bürokraten verbunden. Zum einen steigt der Nutzen des Bürokraten, weil durch das höhere Budget die im vorhergehenden Abschnitt beschriebenen nichtmonetären Vorteile steigen. Zum anderen erhöht sich aber die erwartete Strafe bei Entdeckung der Mittelverschwendung mit steigendem Ausmaß der Abweichung des ausgegebenen Budgets vom für die Arbeit der Bürokratie notwendigen Budget. Aus diesem Grund sinkt der Nutzen der Bürokraten mit steigendem Budget. Die Risikoeinstellung der Bürokraten bestimmt ihr Ausgabeverhalten in erheblicher Weise mit, weil risikoscheue Bürokraten dem zweiten Einfluss eine höhere Wichtigkeit beimessen, so dass ihr Grenzschaden mit steigendem Ressourceneinsatz zunimmt. Risikoscheue Bürokraten werden also im Vergleich zu risikoneutralen Bürokraten eine deutlich geringere Ausweitung des Ressourceneinsatzes vornehmen. ${ }^{160}$

Das fünfte Argument für einen eingeschränkten diskretionären Handlungsspielraum der Bürokraten besteht darin, dass die Bürokraten innerhalb der Verwaltung mobil sind und häufig ihre Arbeitsplätze wechseln. Ein Arbeitsplatzwechsel ist einfacher, wenn der Beschäftigte nur die Abteilung innerhalb einer Bürokratie wechselt, als wenn er von einem privaten Unternehmen zu einem anderen privaten Unternehmen wechselt. Da somit der Wettbewerb um Beförderungen und Versetzungen in der öffentlichen Verwaltung stärker ausgeprägt sei als in der Privatwirtschaft, argumentieren Breton und Wintrobe (1982), dass die Ineffizienz der Bürokratie verringert werde, weil Bürokraten einen Anreiz hätten, sich mit guten Leistungen für weitere Aufgaben zu qualifizieren und eine gute Beziehung zu der hierarchisch über ihnen stehenden politischen Ebene anzustreben. $^{161}$

Sechstens ist zu berücksichtigen, dass die konkrete individuelle Ausprägung der Macht der Bürokraten, wie bei allen anderen Akteuren auch, von ihrer Position in der Verwaltungshierarchie und ihren persönlichen Fähigkeiten abhängt. ${ }^{162}$

Die dargestellten Einschränkungen der bürokratischen Macht spielen eine wesentliche Rolle bei der konkreten Analyse der Beziehungen zwischen Politik und Bürokratie sowie der Untersuchung der institutionellen Ausgestaltung des NIFP, welche in Kapitel 4 vorgenommen werden.

159 Vgl. Mueller (2003), S. 367.

160 Vgl. Mueller (2003), S. 367; Moe (1997), S. 461.

161 Vgl. Breton und Wintrobe (1982), S. 96 f.; Breton (1996), S. 177 f.

162 Vgl. Cox und Jacobsen (2001), S. 108. 


\subsubsection{Alternative Theorieansätze: Risikoaversion und Maximierung des „slack“}

Ein auf dem budgetmaximierenden Bürokraten aufbauender Theorieansatz untersucht die Risikoaversion der Bürokraten und die daraus resultierende Auswahl der von der Bürokratie durchzuführenden Projekte. Diese Theorie besagt, dass Bürokraten aufgrund ihrer Risikoaversion bestimmte Projekte nicht unternehmen, obwohl ihre Finanziers, die Politiker, genau diese Projekte befürworten und durchsetzen würden, falls sie kostenlos die Aktivitäten der Bürokratie überwachen könnten. Auch für diesen Theorieansatz sind somit asymmetrische Informationsverteilung zwischen Politikern und Bürokraten und mangelnde Überwachungsmöglichkeiten entscheidend. Bürokraten möchten möglichst erfolgreiche Projekte unterstützen, damit Politiker ihre Arbeit positiv beurteilen und sie auch für die Zukunft über mindestens das gleiche Budget verfügen lassen. ${ }^{163}$

Ein alternatives Modell von Wyckoff (1990) stellt anstelle der Budgetmaximierung einen vollkommen anderen Anreizmechanismus in den Vordergrund und betont die Wichtigkeit der Bequemlichkeit der Arbeit (,slack“) für den Bürokraten. Der Bürokrat maximiert in diesem Modell nicht das Budget, sondern den „slack“, so dass der Bürokrat bzw. die Verwaltung denjenigen Output herstellt, bei dem die Differenz zwischen erhaltenem Budget und dem für die Produktion des Outputs aufgewendeten Budget maximal wird. Diese Differenz kann für unproduktive Ausgaben verwendet werden, wie z. B. die in Abschnitt 3.3.1 erwähnten nichtmonetären Vorteile. Vom Standpunkt der Politiker aus stellt die Differenz allerdings Ineffizienz dar und kann nur entstehen, weil die Politiker die Kostenfunktionen der Bürokratie zur Produktion des Outputs nicht kennen. Ebenso wie im Modell des budgetmaximierenden Bürokraten ergibt sich als Konsequenz des den „slack“" maximierenden Bürokraten ein größeres von der Bürokratie verwaltetes Budget als die Politiker bei vollständiger Information genehmigen würden. ${ }^{164}$

In der ökonomischen und psychologischen Literatur gibt es diverse weitere Theorieansätze, die sich mit Verhalten und Anreizen der Bürokratie beschäftigen. Ein besonders einflussreicher Beitrag zur Theorie der Bürokratie stammt von Downs (1967), der neben der persönlichen Nutzenmaximierung des Bürokraten auch die Verfolgung altruistischer Motive in seine Analyse mit einbezieht. Bürokraten haben Wertvorstellungen in Bezug auf die von ihnen zu erfüllenden Aufgaben und die dahinter stehenden politischen Ziele. Downs klassifi-

163 Vgl. Gist und Hill (1981), S. 64; Mueller (2003), S. 370 f.; Fölster (1991), S. 36. Dass jegliche Entscheidungsträger bei Evaluation ihrer Arbeit eine stärker risikoscheue Projektauswahl vornehmen, wurde in der Literatur mehrfach gezeigt, vgl. Kahneman und Lovallo (1994), S. $81 \mathrm{f}$.

164 Vgl. Wyckoff (1990), S. 35 ff. 
ziert fünf Bürokratentypen. ${ }^{165}$ Erstens beschreibt er den ehrgeizigen Aufsteiger, der neben seinem Einkommen seine Macht und sein Prestige maximieren will. Deswegen arbeitet dieser entweder auf eine Beförderung in eine Position hin, die mit höherer Reputation verbunden ist, oder bemüht sich um die Verbesserung seiner derzeitigen Position durch eine Budget- und Aufgabenausweitung. Zweitens gibt es den konservativen Bewahrer, der sein derzeitiges Niveau an Einkommen, Prestige und Macht erhalten will. Deswegen verteidigt dieser Bürokratentyp den Status quo und sperrt sich gegen jegliche Veränderung, insbesondere wenn diese mit einer Mittelkürzung für seine Abteilung verbunden ist. Der dritte von Downs beschriebene Bürokratentyp ist der Eiferer, häufig ein Einzelkämpfer, der energisch und aggressiv um sein inhaltliches Anliegen kämpft. Auch kämpft der Eiferer für eine radikale Veränderung des Status quo, wenn diese seinen inhaltlichen Anliegen dient. Viertens gibt es den eng in der Organisation verwurzelten Anwalt, dem seine inhaltlichen Anliegen auch am Herzen liegen, der aber gleichzeitig eine große Loyalität zu seiner Abteilung, seinen Vorgesetzten und seinen Kollegen aufweist. Schließlich beschreibt Downs den Staatsmann als fünften Bürokratentypen, der dem gesamtgesellschaftlichen Wohl dienen möchte und sich nicht für die Einzelinteressen bestimmter Bürokratien interessiert.

Downs' Ansatz bildet zwar aufgrund des hohen Differenzierungsgrads die Realität sehr gut ab, doch kann er keine generalisierbaren Aussagen in Bezug auf das Verhalten und die Anreizsituation der Bürokraten treffen. Bezogen auf den Prozess der Innovationsförderung ist der Typus des Aufsteigers an einer Budget- und Aufgabenausweitung interessiert und ähnelt darin und in seinen persönlichen Zielen dem Bürokraten nach Niskanen. Vom Bewahrer dagegen gehen keine Veränderungsimpulse in Bezug auf das Budget und die Akkumulation neuer Aufgaben aus. Die letzten drei beschriebenen Typen sind wenig berechenbar, ihre Verhaltensweisen hängen von ihrer persönlichen Einschätzung der Sinnhaftigkeit von Innovationsförderung und deren bester Ausgestaltung ab.

Hartig (1990) vermutet, dass in den Verwaltungen, die mit der Vergabe von Subventionen betraut sind, der Typus des Aufsteigers am häufigsten vertreten ist. Denn die Vergabe von Fördermitteln für einzelne Projekte sei mit einem erheblichen Verwaltungsaufwand verbunden, so dass insbesondere diejenigen Bürokratietypen in diese Verwaltungen drängen, die sich eine Gegenleistung versprechen, die ihren Aufwand rechtfertigt. Laut Hartig sind dies vor allem die Aufsteiger, die sich von der Arbeit in einer aufstrebenden Subventionsabteilung in besonderem Maße eine Ausweitung des Budgets, ihrer Kompetenzen und ihres damit verbundenen Prestiges erhoffen und im Gegenzug grundsätzlich eine Expansion der Förderung unterstützen. ${ }^{166}$

165 Vgl. Downs (1967), S. 92 ff.

166 Vgl. Hartig (1990), S. 107 ff. 
Im Gegensatz zu schrumpfenden Subventionsbereichen wie beispielsweise der Steinkohle steigt die Wichtigkeit und Wertschätzung von Innovation und F\&E in der öffentlichen Diskussion. Politiker betonen die Notwendigkeit einer kontinuierlichen Förderung für die Stärkung des Wirtschaftswachstums (vgl. Abschnitt 4.3.2). Das Thema Innovation gilt spätestens seit der 2000 vom Europäischen Rat verabschiedeten Lissabon-Strategie und der Festlegung des $3 \%$ Ziels für F\&E- und Innovationsausgaben als entscheidend für die künftige volkswirtschaftliche Entwicklung europäischer Staaten. Aus diesem Grund wird die unternehmerische Innovationstätigkeit in der EU in hohem Maße staatlich gefördert (vgl. Abschnitt 1.1). Insofern ist die Annahme einleuchtend, dass europäische Bürokraten des Typus Aufsteiger besonders häufig in Positionen in industrie- und innovationspolitischen Abteilungen tätig sind, in deren Aufgabenbereich auch die Innovationsförderung fällt. Da der Aufsteiger nach Downs dem Bürokraten nach Niskanen sehr ähnlich ist, ist Niskanens Verhaltensmodell für die Thematik der Innovationsförderung gut anwendbar.

\subsubsection{Empirische Evidenz}

Die schon erwähnte Schwierigkeit, den Output und die Kostenfunktionen der Bürokratie zu messen, erschwert die empirische Überprüfung der Modelle des budgetmaximierenden, des risikoaversen und das den "slack" maximierenden Bürokraten. Es gibt zahlreiche Studien, die sich mit den Produktionskostendifferenzen ähnlicher Güter und Dienstleistungen zwischen öffentlichen und privaten Unternehmen beschäftigen. Mueller (2003) fasst die Ergebnisse von 71 Studien aus dem Zeitraum 1967-1998 zusammen. 56 Studien kommen zu dem Ergebnis, dass die öffentlichen Anbieter ihre Güter zu höheren Kosten anbieten und ineffizienter produzieren als die privaten Anbieter. Fünf Studien liefern entgegengesetzte Resultate und 10 Studien finden keinen Hinweis auf signifikante Kostendifferenzen. ${ }^{167}$ Auch die Untersuchung der Kosteneffizienz von öffentlichen Schulen in New Yorker Verwaltungsbezirken von Duncombe et al. (1997) kommt zu einem entsprechenden Ergebnis: 88 \% der untersuchten Schulen sind nicht kosteneffizient. ${ }^{168}$ Es gibt also empirisch vielfältige Hinweise darauf, dass eine Produktion durch den öffentlichen Sektor - in Form eines öffentlichen Unternehmens oder einer öffentlichen Verwaltung wie einer Schule $-\mathrm{zu} \mathrm{X-}$ Ineffizienz führt, was als Hinweis auf die Gültigkeit der Modellansätze des budgetmaximierenden und des "slack“ maximierenden Bürokraten interpretiert werden kann.

Daran anknüpfend gibt es empirische Untersuchungen für verschiedene Bereiche der öffentlichen Verwaltung, die untersuchen, in welcher Form die XIneffizienz auftritt. Wie in vorherigen Abschnitt beschrieben, stellt die Theorie

167 Vgl. Mueller (2003), S. $373 \mathrm{ff}$.

168 Vgl. Duncombe et al. (1997), S. 15. 
des risikoaversen Bürokraten einen möglichen Ansatz dar. So finden Gist und Hill (1981) heraus, dass Angestellte der Abteilung für Wohnungsbau und Stadtentwicklung (Department for Housing and Urban Development) in den USA bei der Mittelvergabe im Rahmen eines Stadtentwicklungsprogramms (Urban Development Action Grant) nicht die eigentlich laut Programmzielen zu fördernden, besonders hilfsbedürftigen Gegenden unterstützen. Stattdessen werden eher Projekte unterstützt, für die sich private Kapitalgeber finden, was zwar auf eine höhere Erfolgsquote dieser Projekte schließen lässt, aber nicht den eigentlichen Zielen des Stadtentwicklungsprogramms entspricht. ${ }^{169}$ Die Untersuchung liefert somit Belege für die Theorie des risikoaversen Bürokraten.

Ein Beispiel dafür, dass die Messprobleme ihres Outputs das Verhalten der Bürokraten beeinflussen, stammt von Lindsay (1976) mit seiner Untersuchung der öffentlichen Veteranenkrankenhäuser (Veterans' Adminstration Hospitals). Der Output dieser Krankenhäuser fällt im Vergleich mit dem Output privater Krankenhäuser, z. B. hinsichtlich des Verhältnisses von Krankenhauspersonal zu Patienten, qualitativ schlechter aus. Lindsay führt die Differenzen darauf zurück, dass die Qualität der Patientenbetreuung nicht ohne weiteres empirisch gemessen werden kann und somit die zuständigen Politiker nur Mittel zur Sicherstellung eines Mindestmaßes an Qualität bewilligen. ${ }^{170}$ Dagegen sind private Krankenhäuser aufgrund ihrer Wettbewerbssituation stärker an der Zufriedenheit ihrer Patienten interessiert. Hier wird wieder der Einfluss der Informationsasymmetrie zwischen Finanzier und Bürokratie deutlich. Eine höhere Qualität der Patientenversorgung in den öffentlichen Krankenhäusern wäre zwar eigentlich im Interesse der Politiker, aber da sie diese empirisch nicht überprüfen können, bewilligen sie dafür keine zusätzlichen Mittel. Somit beschränkt sich die zuständige Verwaltung auf die Produktion empirisch überprüfbarer Güter, um positiv evaluiert zu werden und ihr Budget für die Zukunft zu sichern oder zu erhöhen.

Die Studie von Dávila et al. (1999) ergibt bezogen auf einen anderen Verwaltungsbereich das gleiche Resultat. Die Autoren untersuchen die Immigrationskontrollen in den USA und stellen fest, dass die Kontrollen an den Außengrenzen der USA deutlich intensiver durchgeführt werden als die Kontrollen innerhalb der USA bei Unternehmen, die illegale Einwanderer beschäftigen könnten. Obwohl das Optimum in einer ausgewogenen Strategie besteht und die Außenkontrollen einen geringeren Abschreckungseffekt auf Immigrationswillige ausüben als Innenkontrollen, wird den Außenkontrollen der Vorzug gegeben. Die Autoren erklären dieses Phänomen dadurch, dass die Gewährung des Budgets vom vorzuweisenden Erfolg im Sinne der Anzahl der festgenommenen illegalen Immigranten besteht und nicht von der eigentlichen Zielgröße der Politik,

169 Vgl. Gist und Hill (1981), S. $70 \mathrm{ff}$.

$170 \mathrm{Vgl}$. Lindsay (1976), S. $1068 \mathrm{ff}$. 
nämlich der nicht zu messenden Anzahl der sich bereits im Land befindlichen illegalen Immigranten. Da Außenkontrollen eher zu einer Erhöhung der Anzahl der Festgenommenen führen als Innenkontrollen und der größere Abschreckungseffekt der Innenkontrollen nicht quantifiziert und den Kontrolleuren zugerechnet werden kann, präferiert die zuständige Verwaltung (U.S. Immigration and Naturalization Service) die weniger effektiven Außenkontrollen. ${ }^{171}$

\subsubsection{Fazit}

Die Neue Institutionenökonomik liefert eine eigene Theorie der Bürokratie, weil die Bürokratie sich in bestimmten Charakteristika grundlegend von anderen Organisationen unterscheidet. Niskanen definiert die Bürokratie als eine nicht auf Gewinn ausgerichtete Organisation, deren Einkommen nicht (nur) über den Verkauf ihres Outputs, sondern auch über wiederkehrende externe Finanzmittel erfolgt. Im Gegensatz zu Politikern sind Bürokraten weder in ihrer Ernennung noch in ihrer Arbeit von der Wählermeinung abhängig. Ihr Entscheidungsspielraum bestimmt sich vielmehr in ihrer Beziehung zu den Politikern, die Regeln beschließen, deren konkrete Umsetzung den Bürokraten obliegt. Aufgrund einer Informationsasymmetrie zwischen gut informierten Bürokraten mit Expertenwissen in ihrem Bereich und den sie überwachenden Politikern ist die Macht der Bürokraten nicht unerheblich. Das in der Literatur vorherrschende Modell ist der budgetmaximierende Bürokrat nach Niskanen, der seinen Informationsvorsprung dafür nutzt, die Größe und das Budget seiner Abteilung zu maximieren. Damit verbunden ist die Maximierung der X-Ineffizienz, nämlich den nichtmonetären persönlichen Vorteilen des Bürokraten. Es findet sich in der Literatur breite empirische Evidenz, die die Theorie des budgetmaximierenden Bürokraten stützt.

Neben dem budgetmaximierenden Bürokraten wurden drei weitere Theorieansätze vorgestellt: der risikoaverse Bürokrat, der „slack“ maximierende Bürokrat und die Bürokratentypologie von Downs. Der erste Ansatz beschreibt die bewusste risikoaverse Projektauswahl durch Bürokraten, die von Politikern am Erfolg der von ihnen ausgewählten Projekte gemessen werden. Die risikoaverse Projektauswahl wird auch vorgenommen, wenn eine risikoreichere Projektauswahl eher im Interesse der Politiker wäre. Einige empirische Studien stützen diesen Theorieansatz, der in Abschnitt 4.4.2 in Bezug auf die Projektauswahl im Rahmen von Innovationsförderprogrammen wieder aufgegriffen wird. Das Modell der Maximierung des „slack“ beschreibt die Maximierung der Bequemlichkeit der Arbeit durch den Bürokraten und weicht nur graduell vom budgetmaximierenden Bürokraten ab. Die Typologie der Bürokratie nach Downs stellt dagegen einen sehr differenzierten Ansatz dar und beschreibt mit dem Aufsteiger, dem Bewahrer, dem Eiferer, dem Anwalt und dem Staatsmann fünf verschiede-

171 Vgl. Dávila et al. (1999), S. 336 ff. 
ne Bürokratietypen, denen sowohl ihre persönliche Nutzenmaximierung als auch altruistische Motive am Herzen liegen. Es wurde dargelegt, warum der budgetmaximierende Bürokrat und der Aufsteiger in für Innovationsförderung zuständigen Abteilungen häufig zu finden sein sollte und deswegen die für diese Arbeit wesentlichen Bürokratietypen darstellen.

Alles in allem sollte die Entscheidungsgewalt der Bürokraten aus mehreren Gründen prinzipiell nicht überschätzt werden: Erstens besitzen Bürokraten in der Realität keine vollkommene Monopolposition. Zweitens erwarten Politiker die Erfüllung konkreter Outputvorgaben und informieren sich über die konkrete Mittelverwendung der Bürokratie mit Hilfe spezieller Überwachungsorganisationen. Drittens konkurrieren verschiedene Verwaltungen miteinander um Aufgaben und Aufträge. Viertens besteht jede Bürokratie aus einzelnen Individuen, deren persönliche Zielsetzung den Zielen der Bürokratie entgegenstehen kann.

\subsection{Zusammenfassung der Ergebnisse}

Das Ziel dieses Kapitels ist die Vorstellung und Diskussion derjenigen Theorieansätze der Neuen Institutionenökonomik, die für die konkrete Analyse des NIFP im Folgenden benötigt werden. In diesem Kapitel wurde zunächst dargestellt, warum das Konzept der Institution auf das Innovationsförderprogramm anwendbar ist. Ein Förderprogramm besteht aus einer Gesamtheit von Regeln zur Auswahl der geförderten Unternehmen und zur Durchführung der Fördermaßnahmen. Diese Regeln beeinflussen das Verhalten der beteiligten Individuen, die sich den Akteursgruppen Politikern, Bürokraten und Unternehmern zuordnen lassen. Institutionelle Durchsetzungsmechanismen bestehen in der Auswahl der zu fördernden Unternehmen und der Überprüfung der Fördermittelverwendung. Die Annahme des methodologischen Individualismus besagt, dass Institutionen unter Rückgriff auf die beteiligten Individuen zu erklären sind. Deswegen stellt diese Arbeit die am NIFP beteiligten Akteure mit ihren Interessen, Anreizen und Verhaltensweisen in den Mittelpunkt der Analyse.

Eine grundlegende Annahme der Neuen Institutionenökonomik besteht in der Berücksichtigung von unvollständiger Information. Ein Wirtschaftssubjekt kann nicht alle relevanten Informationen aufnehmen und in seine Entscheidungsfindung mit einbeziehen. Das Modell des Homo Oeconomicus wird für eine realitätsnähere Analyse durch die Annahme der „bounded rationality“ ergänzt. Es geht Wirtschaftsubjekten nicht um die Erzielung des optimalen Ergebnisses, sondern um das Erreichen eines akzeptablen Ergebnisses. Aus der unvollständigen Information ergeben sich die Existenz von Transaktionskosten und die Prinzipal-Agenten-Beziehung als wesentliche Bestandteile der Neuen Institutionenökonomik. 
Neben diesen Annahmen, die für alle am Innovationsfördersystem beteiligten Wirtschaftssubjekte gleichermaßen gelten, wurden spezielle Theorien für die Akteursgruppen der Politiker und der Bürokraten vorgestellt. Für die Politiker ist die Theorie der Neuen Politischen Ökonomie anzuwenden, die den individuellen Nutzen der Politiker explizit berücksichtigt. Eine gezielte Beeinflussung der wichtigsten wirtschaftlichen Variablen soll den Amtsinhabern die Wiederwahl ermöglichen und ihnen die mit der politischen Macht verbundenen positiven Faktoren wie Einkommen und Prestige weiterhin sichern. Außerdem wurde auf die mögliche Beeinflussung der politischen Amtsinhaber durch Lobbygruppen im Rahmen des „rent seeking“ eingegangen. So könnte von Interessengruppen, die für Innovationsförderprogramme potentiell in Frage kommende Unternehmer vertreten, der Versuch unternommen werden, Politiker zu einer Ausweitung der Innovationsförderung zu drängen, weil einzelne Interessengruppenmitglieder von der Förderung profitieren könnten.

Das Verhalten der Bürokraten wird durch die Theorie der Bürokratie analysiert. Die Bürokratie zeichnet sich demzufolge durch ihren nicht marktfähigen Output und die Informationsvorteile aus, die sie gegenüber den Politikern besitzt. Da die Überwachung der Bürokratie durch die Politik sehr schwierig ist, vermutet die Theorie der Bürokratie, dass Bürokraten neben ihren offiziellen Zielen relativ ungehindert ihre eigenen Ziele verfolgen können. Der bis heute einflussreichste Theorieansatz stammt von Niskanen und unterstellt den Bürokraten vordringlich an der Maximierung des Budgets ihrer Abteilung interessiert zu sein. Mit einem höheren Budget steigt die X-Ineffizienz und somit der Nutzen der Bürokraten. Da die für Innovationsförderung zuständigen Abteilungen zu den expandierenden Abteilungen innerhalb der Bürokratie zählen, finden sich hier besonders viele Bürokraten im Niskanen'schen Sinne, die in der Bürokratentypologie von Downs als Aufsteiger bezeichnet werden. Diese Bürokraten sehen in expandierenden Abteilungen die Möglichkeit, ihren persönlichen Nutzen durch eine Erhöhung von Budget und Zuständigkeiten zu erhöhen. Dieser Bürokratentyp ist in den für Wirtschaftsförderung zuständigen Abteilungen besonders stark vertreten und wird deswegen im Rahmen der institutionenökonomischen Analyse in Kapitel 4 als Verhaltensmodell verwendet. Die Theorie führt trotz der mit ihr verbundenen Vereinfachungen zu aufschlussreichen Aussagen hinsichtlich des Entscheidungsverhaltens der Bürokraten im Rahmen ihrer von der Politik vorgegebenen Grenzen. So stimmen Theorie und Empirie darin überein, dass budgetmaximierende Bürokraten eine eher risikoaverse Auswahl der von der Bürokratie durchzuführenden Projekte vornehmen. Denn diese Auswahl verbessert ihre Erfolgsbilanz und sichert der Bürokratie das Wohlwollen der Politiker und somit das Budget für ihren Aufgabenbereich bzw. ihre Abteilung.

Es gibt jedoch einige Gründe für die Annahme, dass die Macht und Entscheidungsgewalt der Bürokraten in der Realität tatsächlich eingeschränkter ist, 
als in den Bürokratietheorien vorausgesetzt wird. $\mathrm{Zu}$ diesen Gründen zählen eine starke Machtstellung der Politiker, der mögliche Wettbewerb verschiedener Bürokratien um Aufgaben und das Beförderungsstreben vieler Bürokraten. Die nähere Untersuchung der Entscheidungsgewalt der Bürokraten und der Beziehung zwischen Bürokratie und Politik ist für eine institutionenökonomische Analyse, wie sie im folgendem Kapitel vorgenommen wird, unerlässlich, um die tatsächliche Macht der Bürokratie im NIFP zu untersuchen.

Im Gegensatz zu den Politikern und Bürokraten gibt es für Unternehmer im Rahmen der Neuen Institutionenökonomik keine eigene Theorierichtung, weswegen Unternehmer in diesem Kapitel nicht gesondert aufgeführt wurden. Unternehmer werden als ganz normale Wirtschaftssubjekte betrachtet, die den allgemeinen Einschränkungen wie der „bounded rationality“ und Informationsasymmetrien unterliegen und die sich in Prinzipal-Agent-Beziehungen wieder finden, wie sie im folgenden Kapitel am Beispiel des NIFP beschrieben werden. 


\section{Institutionenökonomische Untersuchung des Niedersächsischen Innovationsförderprogramms}

Dieses Kapitel wendet die allgemeinen Ergebnisse der Theorie der Neuen Institutionenökonomik des vorigen Kapitels auf die spezielle Problematik des NIFP an. Es erfolgt eine Untersuchung des Programmablaufs und der Rolle der verschiedenen daran beteiligten Akteursgruppen. Bei dieser Untersuchung steht die Frage im Mittelpunkt, welche Unternehmen und Unternehmer durch das NIFP tatsächlich gefördert werden. Drei Akteursgruppen sind direkt am NIFP beteiligt: die regionalen Politiker, die Bürokraten des niedersächsischen Ministeriums für Wirtschaft, Arbeit und Verkehr (im Folgenden Wirtschaftsministerium) und der anderen am Programmablauf beteiligten Organisationen sowie die geförderten Unternehmer. Doch müssen sich alle diese Akteure mit ihrem Verhalten nach dem rechtlichen Rahmen richten, in welchem Wirtschaftsförderung in der EU und also auch in Niedersachsen stattfinden darf. Deswegen wird im folgenden Abschnitt 4.1 der europarechtliche Rahmen von Wirtschaftsförderung im Allgemeinen und Innovationsförderung im Besonderen betrachtet. Es folgt ein Überblick über die institutionelle Ausgestaltung des NIFP in Abschnitt 4.2. Daran anschließend werden die im Rahmen des NIFP auftretenden Prinzipal-AgentBeziehungen erläutert. Die Anreize und Interessen der einzelnen Akteursgruppen werden mit Hilfe der theoretischen Erkenntnisse aus Kapitel 3 analysiert. Zunächst geht es in Abschnitt 4.3 um die Rolle der Politiker, daran anschließend in Abschnitt 4.4 um die verschiedenen Bürokratengruppen, die in den Innovationsförderprozess involviert sind, und in Abschnitt 4.5 um die geförderten Unternehmer. Abschließend werden in Abschnitt 4.6 die Ergebnisse und Hypothesen dieses Kapitels kurz zusammengefasst.

\subsection{Der europarechtliche Rahmen von Wirtschaftsförderung}

Jede Form von Wirtschaftsförderung in Europa findet innerhalb des Systems von Wettbewerbsregeln statt, das die EU zum Schutz ihres Gemeinsamen Marktes vor Verfälschungen und Beeinträchtigungen des gemeinschaftlichen Handels erlassen hat. Die fortschreitende europäische Integration führt sowohl zu einem Abbau der protektionistischen Schranken zwischen den Mitgliedstaaten als auch zu einem steigenden Standortwettbewerb zwischen Staaten und Regionen. Wirtschaftsförderung in Form von staatlichen Beihilfen gewinnt als Instrument der Standortpolitik immer mehr an Bedeutung. Da wohlhabende Regionen in stärkerem Maße staatliche Beihilfen gewähren können als ärmere Regionen, besteht die Gefahr, dass die wohlhabenden Regionen durch ihre Förderpolitik immer 
attraktiver für Unternehmensansiedlungen werden und mit den zu erhaltenden zusätzlichen Einnahmen aus dieser Ansiedlung ihre Standortvorteile weiter ausbauen können. ${ }^{172}$ Die Divergenz zwischen den Regionen würde sich als Konsequenz verschärfen, was im Gegensatz zu den Aufgaben der EU steht, zu denen es u. a. gehört, ,eine harmonische, ausgewogene und nachhaltige Entwicklung des Wirtschaftslebens" sowie ,einen hohen Grad von Wettbewerbsfähigkeit und Konvergenz der Wirtschaftsleistungen" zu fördern (Art. 2 EG-Vertrag). Aus diesem Grunde gehört zum System der europäischen Wettbewerbsregeln das Instrument der Beihilfenkontrolle.

Gemäß Art. 87 Abs. 1 EG-Vertrag gilt ein präventives Beihilfenverbot mit Erlaubnisvorbehalt: ,Soweit in diesem Vertrag nicht etwas anderes bestimmt ist, sind staatliche oder aus staatlichen Mitteln gewährte Beihilfen gleich welcher Art, die durch die Begünstigung bestimmter Unternehmen oder Produktionszweige den Wettbewerb verfälschen oder zu verfälschen drohen, mit dem Gemeinsamen Markt unvereinbar, soweit sie den Handel zwischen Mitgliedstaaten beeinträchtigen. "In dieser Vorschrift werden fünf Tatbestandsmerkmale einer Beihilfe genannt, die kumulativ vorliegen müssen: ${ }^{173}$ Erstens besteht die Beihilfe aus einem Transfer staatlicher Mittel, wobei es sich hier um einen direkten Transfer staatlicher Mittel oder um einen indirekten Transfer durch beispielsweise einen Verzicht des Staates auf ihm zustehende Einnahmen handeln kann. Zweitens handelt es sich bei einer Beihilfe um eine Begünstigung, was bedeutet, dass dem staatlich gewährten Vermögensvorteil keine marktadäquate Gegenleistung gegenübersteht. Drittens wird die Begünstigung nur bestimmten Unternehmen oder Produktionszweigen gewährt, also profitiert nur eine ausgewählte Gruppe von der Beihilfe. Viertens droht eine Wettbewerbsverfälschung durch die Beihilfe, weil sich die Wettbewerbsbedingungen für die Empfänger der Beihilfe gegenüber der Konkurrenz verbessern. Fünftens muss der Handel zwischen zwei Mitgliedsstaaten durch die Beihilfe beeinträchtigt werden, dadurch dass die Stellung des begünstigten Unternehmens im Handel verstärkt wird. Durch den Gemeinsamen Markt der EU wird allerdings in der Regel davon ausgegangen, dass diese Beeinträchtigung des Handels vorliegt. Die Förderung von Unternehmen im Rahmen des NIFP stellt somit eine Beihilfe dar.

Ausnahmen vom Beihilfenverbot sind in Art. 87 Abs. 2 und 3 EG-Vertrag vorgesehen, wobei es sich entweder um Legal- oder um Ermessensausnahmen handeln kann, über die die Europäische Kommission entscheidet (Art. 88 EGVertrag). Darüber hinaus kann die Kommission durch so genannte Gruppenfreistellungsverordnungen bestimmte Beihilfearten für mit dem Gemeinsamen

172 Vgl. Haucap und Hartwich (2006); Jaenichen und Steinrücken (2007), S. 417 f.

173 Vgl. Frenz (2007), Rn. 30 ff.; Götz und Martínez Soria (2007), Rn. 49 ff.; Cremer (2007), Rn. 9 ff. 
Markt vereinbar erklären. ${ }^{174}$ Auf diese Weise können Beihilfen im Bereich F\&E bzw. Innovation dazu beitragen, die in Kapitel 1 beschriebenen Ziele der Lissabon-Strategie zu erreichen. In diesem Sinne wurde das NIFP/F\&E von der Europäischen Kommission als Ermessensausnahme vom Beihilfenverbot nach Art. 87 Abs. 3 c) EG-Vertrag („Förderung der Entwicklung gewisser Wirtschaftszweige") zugelassen. ${ }^{175}$ Das NIFP/Handwerk fällt dagegen unter die Gruppenfreistellungsverordnung für „De-minimis“-Beihilfen ${ }^{176}$, wonach Beihilfen unterhalb eines bestimmten Schwellenwertes nicht von der Kommission genehmigt werden müssen, sowie unter die Gruppenfreistellungsverordnung für Ausbildungsbeihilfen ${ }^{177}$ (vgl. die Ziffern 6.2 und 6.3 der NIFP-Richtlinie).

Die Finanzierung des NIFP ist ebenfalls in den Rahmen der EU eingebunden. So stammen die Mittel des Programms nicht nur aus dem niedersächsischen Landeshaushalt, sondern es erfolgt eine Kofinanzierung aus EFRE-Mitteln. In der Förderperiode 2000-2006 dienten die EFRE-Mittel zur Förderung von Vorhaben in Gebieten, die unter das Ziel 2 des EFRE („Unterstützung der wirtschaftlichen und sozialen Umstellung der Gebiete mit Strukturproblemen") fielen. In der Förderperiode 2007-2013 können die EFRE-Mittel im Rahmen der Ziele „Regionale Wettbewerbsfähigkeit und Beschäftigung“ und „Konvergenz“ landesweit eingesetzt werden. ${ }^{178}$

Im folgenden Abschnitt wird die institutionelle Ausgestaltung des NIFP und sein organisatorischer Ablauf detailliert vorgestellt. Im Rahmen der rechtlichen Vorgaben der EU können regionale Politiker weitgehend autonom darüber entscheiden, welche Wirtschaftsförderprogramme sie entwerfen und wie sie diese ausgestalten. Warum sie speziell ein Interesse an der Durchsetzung von Innovationsförderprogrammen haben, wird in Abschnitt 4.3 näher erläutert.

174 Art. 1 Abs. 1 der EG-Verordnung Nr. 994/98 vom 07.05.1998 (ABI. EG Nr. L 142 vom 14.05.1998, S. 1). Vgl. hierzu insgesamt Frenz (2007), Rn. 763 ff.; Götz und Martínez Soria (2007), Rn. $144 \mathrm{ff}$.

175 Vgl. Kommissionsdokument C(2004)1663 vom 22.04.2004, in: http://ec.europa.eu/ community_law/state_aids/comp-2004/n041-04.pdf, zugegriffen am 05.08.2008.

176 EG-Verordnung Nr. 69/2001 vom 12.01.2001 (ABl. EG Nr. L 10 vom 13.01.2001, S. 30).

177 EG-Verordnung Nr. 68/2001 vom 12.01.2001 (ABl. EG Nr. L 10 vom 13.01.2001, S. 20).

178 Vgl. die Informationen zur EFRE-Förderung der beiden Förderperioden auf der Internetseite des niedersächsischen Wirtschaftsministeriums, in: http://www.mw.niedersachsen.de/master/C44260858_N35379853_L20_D0_I712.html, zugegriffen am 05.08 . 2008. 


\subsection{Institutionelle Ausgestaltung des NIFP}

Das NIFP besteht aus zwei Teilen, zum einen aus dem niedersächsischen Innovationsförderprogramm für Forschungs- und Entwicklungsausgaben (NIFP/F\&E), zum anderen aus dem niedersächsischen Innovationsförderprogramm für das Handwerk (NIFP/Handwerk). Für beide Teilprogramme gilt, dass die Unternehmen zunächst eine Projektskizze (8-10 Seiten) bei der Investitionsund Förderbank Niedersachsen (NBank) einreichen müssen und nach einer positiven Beurteilung dieser Skizze zur Antragstellung aufgefordert werden. ${ }^{179}$

Das NIFP/F\&E fördert F\&E-Vorhaben in den Bereichen industrielle Forschung (Gewinnung von Erkenntnissen für neue Produkte, Dienstleistungen und Produktionsprozesse) und vorwettbewerbliche Entwicklung (Umsetzung der Erkenntnisse der industriellen Forschung). Besondere Schwerpunkte liegen in den Bereichen Energieerzeugung und -einsparung, erneuerbare Energien sowie Informations- und Kommunikationstechnologien (Ziffer 2.1 der NIFP-Richtlinie). Die NBank weist in den Programminformationen zwar explizit darauf hin, dass sich die Förderung auf die Schwerpunkttechnologiefelder des Landes Niedersachsen konzentriert (Adaptronik, Brennstoffzelle, Life Sciences, Mikrosystemtechnik und Sensorik, Nano- und Materialinnovationen, Telematik). ${ }^{180}$ Jedoch stammt in der Praxis nur ein Teil der geförderten Projekte aus diesen Technologiefeldern. ${ }^{181}$ Die Förderung steht somit allen Unternehmen offen, die Innovationsprojekte in sämtlichen Bereichen industrieller Forschung und vorwettbewerblicher Entwicklung planen, sofern ihre Innovationsvorhaben eine deutschlandweite Neuheit darstellen (Ziffer 4.1 der NIFP-Richtlinie).

Wie in Abbildung 8 dargestellt, sind folgende Akteure auf der Seite des Landes am NIFP/F\&E beteiligt: Während die niedersächsische Landesregierung über die allgemeine politische Ausrichtung der Wirtschaftsförderung entscheidet, hat das Wirtschaftsministerium zusammen mit dem Umweltministerium 2004 die NIFP-Richtlinie über die Vergabe der Fördermittel verfasst (vgl. Abschnitt 4.3.1). Auf dieser Grundlage sowie unter Berücksichtigung der Evaluationen der NBank (verantwortlich für den formalen Evaluationsteil und die Mittelverfügbarkeit im Rahmen des Programms) und des Innovationszentrums Niedersachsen (verantwortlich für den technischen und wirtschaftlichen Evaluationsteil) entscheiden die Mitarbeiter des Wirtschaftsministeriums über die För-

179 Vgl. Programmflyer des NIFP/F\&E auf der Internetseite der NBank, in: http:// www.nbank.de/_downloads/Foerderprograme/Innovations_Forschung_Entwicklung/ Programmflyer.pdf, zugegriffen am 02.08.2008.

180 Vgl. Merkblatt der NBank „Innovationsförderprogramm A. Förderung von Forschungsund Entwicklungsvorhaben“, in: http://www.nbank.de/_downloads/Foerderprogramme/ Innovations_Forschung_Entwicklung/Produktinformation.pdf, zugegriffen am 02.08. 2008.

181 Die Angaben wurden der Verfasserin von der NBank übermittelt. 
deranträge. Nach Auskunft der Beteiligten folgt das Wirtschaftsministerium in der Regel den Förderempfehlungen der NBank und des Innovationszentrums und weicht nur in Ausnahmefällen davon ab. Die Fördermittel werden dann von der NBank ausgegeben.

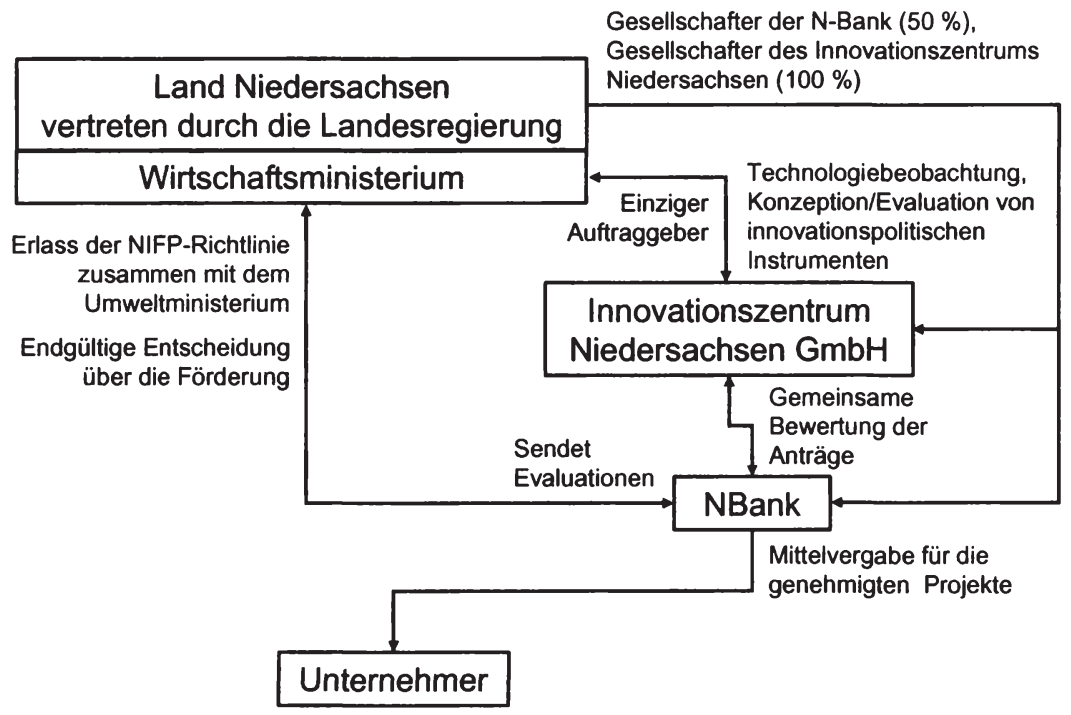

Abbildung 8: Akteure des NIFP/F\&E (Eigene Darstellung).

Laut Ziffer 5 der NIFP-Richtlinie werden die Fördermittel grundsätzlich in Form eines rückzahlbaren Darlehens gewährt. Nur in Ausnahmefällen soll die Förderung in Form eines nicht rückzahlbaren Zuschusses erfolgen und zwar dann „wenn durch die Gewährung eines Darlehens der Förderzweck nicht erreicht werden kann". In der Praxis werden jedoch bislang ausschließlich Zuschüsse vergeben, wohl auch aus Gründen der höheren Attraktivität des Programms für die zu fördernden Unternehmen. Die Zuschüsse im Rahmen des NIFP/F\&E betragen bis zu $25 \%$ der projektbezogenen zuwendungsfähigen Ausgaben für Einzel- und Verbundvorhaben sowie $35 \%$ für Kooperationsvorhaben. Der Fördersatz kann unter bestimmten Bedingungen auf bis zu $50 \%$ ansteigen, nämlich sofern es sich bei den geförderten Unternehmen um KMU handelt, diese in GAFördergebieten ${ }^{182}$ ansässig sind oder Ziele eines spezifischen Programms ver-

182 Die GA-Fördergebiete werden im Rahmen der Gemeinschaftsaufgabe „Verbesserung der regionalen Wirtschaftsstruktur" (GA) von Bund und Ländern gemeinsam festgelegt, vgl. http://www.bmwi.de/BMWi/Navigation/Wirtschaft/Wirtschaftspolitik/Regionalpoli tik/gemeinschaftsaufgabe.html, zugegriffen am 02.08.2008. 
wirklicht, das unter das FTE-Rahmenprogramm der EU ${ }^{183}$ fällt. Die Förderhöchstsumme liegt bei Vorhaben der Informations- und Kommunikationstechnologie bei $200.000 €$, bei Verbundvorhaben erhöht sich die Förderhöchstsumme auf $500.000 €$, höchstens jedoch $200.000 €$ pro Verbundpartner. ${ }^{184}$ Für Fördervorhaben anderer Bereiche gibt es keine Förderhöchstgrenze in diesem Programmteil.

Von 2004 bis 2007 haben 145 Unternehmen eine Bewilligung für den Erhalt von Fördermitteln im Rahmen des NIFP/F\&E bekommen. Die Anzahl der insgesamt eingegangenen Projektskizzen wird von der NBank auf 500 geschätzt. Die Bewilligungen erhielten 92 kleine und 20 mittlere Unternehmen sowie 33 Unternehmen, die keine KMU darstellen. Das Fördervolumen lag bei insgesamt 54,30 Mio. Euro. 16 Unternehmen haben mehrfach Fördermittel erhalten. ${ }^{185}$

Handwerksunternehmen steht neben dem NIFP/F\&E zusätzlich das NIFP/Handwerk offen (Ziffer 2.2 der NIFP-Richtlinie), welches Forschungsvorhaben, bei denen Erkenntnisse über neue Produkte, Dienstleistungen und Produktionsverfahren gewonnen werden sollen, und Entwicklungsvorhaben fördert. Die geförderten Vorhaben im Handwerk müssen laut Ziffer 4.2 der Richtlinie anders als im F\&E-Programmteil - nur den unternehmensbezogenen Stand der Technik übertreffen. Jedoch werden in der Realität deutlich härtere Maßstäbe angelegt. ${ }^{186} \mathrm{Im}$ Handwerksprogrammteil können auch Maßnahmen zur besseren Vermarktung gefördert werden, sofern sie im Zusammenhang mit den oben erwähnten innovativen F\&E-Vorhaben zu einer Absatzsteigerung, einer Arbeitsplatzsicherung oder einem Arbeitsplatzausbau beitragen können. Auch besondere Qualifizierungsvorhaben und die Entwicklung von innovativen technischen und organisatorischen Kooperationsmodellen können gefördert werden. Die Förderprojekte können entweder von den Handwerksunternehmen allein oder in Zusammenarbeit mit niedersächsischen Hochschulen und anderen Forschungseinrichtungen durchgeführt werden. Das NIFP/Handwerk ist deutschlandweit das einzige Innovationsförderprogramm, das sich speziell an die Zielgruppe Handwerk richtet. ${ }^{187}$

183 Die EU-Forschungsrahmenprogramme (Art. 166 EG-Vertrag) fassen spezifische EUProgramme für Forschung und technologische Entwicklung in einem zeitlich befristeten Rahmen zusammen, vgl. http://europa.eu/scadplus/leg/de/s23001.htm, zugegriffen am 02.08.2008.

184 Vgl. Merkblatt der NBank ,Innovationsförderprogramm A. Förderung von Forschungsund Entwicklungsvorhaben“, in: http://www.nbank.de/_downloads/Foerderprogramme/ Innovations_Forschung_Entwicklung/Produktinformation.pdf, zugegriffen am 02.08. 2008.

185 Die Angaben wurden der Verfasserin von der NBank übermittelt.

186 Vgl. Gespräch mit Herm Strunk-Lissowski am 28.03.2008 in Anhang A.

187 Vgl. Astor et al. (2006), S. 107. Eine Recherche in der Förderdatenbank des Bundes (http://www.foerderdatenbank.de) vom 25.06.2008 ergab dasselbe Resultat. 
Wie in Abbildung 9 deutlich wird, unterscheidet sich das NIFP/Handwerk in der Ausgestaltung vom NIFP/F\&E insofern, als dass die Evaluation der Anträge nicht durch das Innovationszentrum Niedersachsen, sondern durch den Innovationsberater der regional zuständigen Handwerkskammer erfolgt (Ziffer 7.3 der NIFP-Richtlinie). Nach jeder Bewerbungsrunde findet ein Treffen der Innovationsberater mit jeweils einem Vertreter des Heinz-Piest-Instituts für Handwerkstechnik an der Leibniz Universität Hannover, des Innovationszentrums, der Vereinigung der Handwerkskammern Niedersachsen, des Wirtschaftsministeriums und der NBank statt. Diese Runde beschließt die Förderempfehlungen, wobei ca. $90 \%$ der in dieser Runde vorgelegten Förderanträge zur Förderung empfohlen werden. Die Selektion der Anträge erfolgt vorher während des Prozesses der Antragstellung, in dem die Innovationsberater den Unternehmen direkt von einer Bewerbung abraten, wenn ihrer Einschätzung nach die Projektidee bzw. der Antrag keine Chance auf Förderung hat oder keine Programmmittel in ausreichender Höhe vorhanden sind. ${ }^{188}$ Anschließend prüft die NBank noch die formale Förderfähigkeit und die Empfehlungen werden an das Wirtschaftsministerium weitergeleitet, welches in der Regel den Förderempfehlungen folgt.

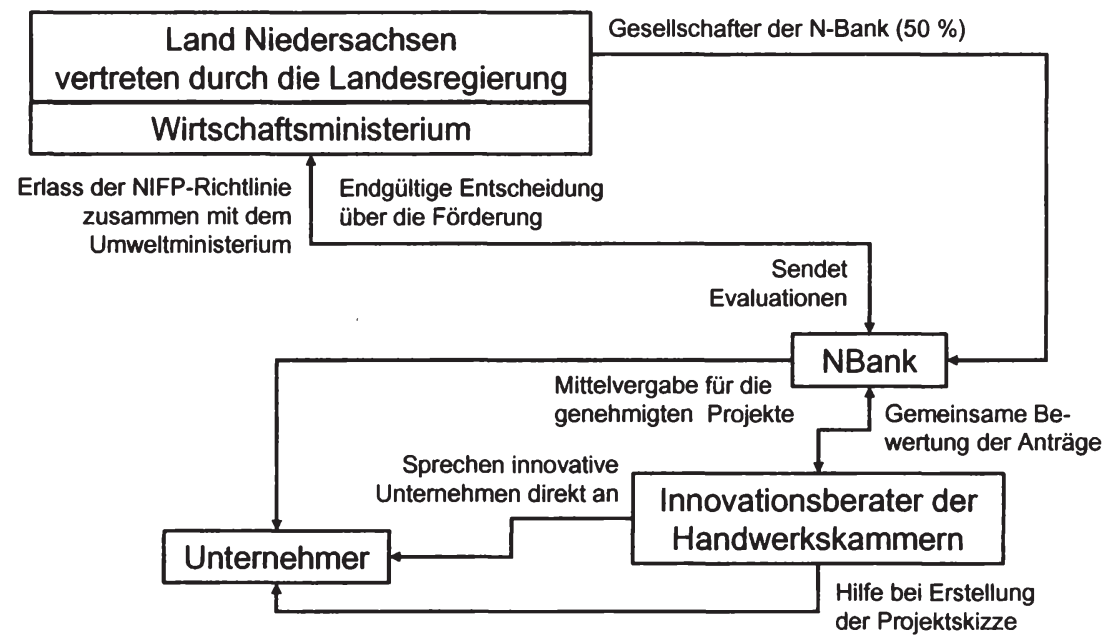

Abbildung 9: Akteure des NIFP/Handwerk (Eigene Darstellung).

Ein weiterer wesentlicher Unterschied der beiden Programmteile besteht darin, dass es für das Handwerk nur zwei Termine im Jahr gibt, zu denen Projektskizzen eingereicht werden können, während dies beim NIFP/F\&E jederzeit möglich

188 Vgl. Gespräch mit Herm Strunk-Lissowski am 28.03.2008 in Anhang A. 
ist. Allerdings gibt es im Jahr 2008 voraussichtlich keine Bewerbungstermine für das NIFP/Handwerk, da die Fördermittel des Jahres durch im November 2007 erstellte Zuwendungsbescheide vollständig belegt wurden. ${ }^{189}$

Die Fördermittel des NIFP/Handwerk werden ebenfalls in Form eines nicht rückzahlbaren Zuschusses in Höhe von maximal $35 \%$ der zuwendungsfähigen Ausgaben gewährt. Wenn eine Forschungseinrichtung am Förderprojekt beteiligt ist, können bis zu $50 \%$ der auf die Forschungseinrichtung entfallenden förderfähigen Ausgaben gefördert werden. Wenn die förderfähigen Ausgaben der Forschungseinrichtung höher als $25 \%$ der förderfähigen Gesamtausgaben sind, kann der Fördermittelempfänger (also das Handwerksunternehmen) für seine Ausgaben einen Zuschuss von bis zu $50 \%$ erhalten. Die Förderhöchstsumme liegt bei $50.000 €$, in Ziel-2-Gebieten bei $100.000 €$. $^{190}$

Von 2004 bis 2007 haben 96 Unternehmen eine Bewilligung für den Erhalt von Fördermitteln im Rahmen der Innovationsförderung im Handwerk bekommen. Die Bewilligungen erhielten 83 kleine und 12 mittlere Unternehmen sowie ein Unternehmen, das kein KMU darstellt. Das Fördervolumen lag bei insgesamt 7,62 Mio. Euro. ${ }^{191}$ Die Zahlen weisen auf das deutlich geringere Fördervolumen pro Projekt im Vergleich zum F\&E-Programmteil hin.

\subsection{Die Rolle der Politiker}

Im Folgenden wird eine institutionenökonomische Untersuchung des NIFP vorgenommen. Begonnen wird dabei mit der Rolle der regionalen Politiker, wobei zunächst konkret ihre Kompetenzen und ihre Einbindung in das NIFP betrachtet werden (Abschnitt 4.3.1), bevor anschließend auf das generelle Interesse der Politiker an Innovationsförderung und die daraus resultierenden Schlussfolgerungen für das Programm eingegangen wird (Abschnitt 4.3.2).

189 Vgl. die Antwort von Wirtschaftsminister Hirche auf die mündliche Anfrage der Abgeordneten Möhrmann, Lies, Schminke, Schneck, Schostok, Siebels, Tippelt und Will (SPD) in der Sitzung des Niedersächsischen Landtages am 03.07.2008, in: http:// www.mw.niedersachsen.de/master/C48244757_L20_D0_I712_h1.html, zugegriffen am 02.08.2008.

190 Vgl. Merkblatt der NBank „Innovationsförderprogramm B. Innovationsförderung im niedersächsischen Handwerk“", in: http://www.nbank.de/service/doc_unterlagen/IN/produktinformation_handwerk.pdf, zugegriffen am 10.02.2007. Wegen der oben erwähnten Fördermittelknappheit für 2008 steht zur Zeit kein Merkblatt für das NIFP/Handwerk auf der Internetseite der NBank zur Verfügung.

191 Die Angaben wurden der Verfasserin von der NBank übermittelt. 


\subsubsection{Die Rolle der regionalen Politiker im NIFP}

In Ergänzung zu der in Abschnitt 3.2 verwendeten Definition können Politiker im engeren Sinne als diejenigen verstanden werden, ,, die befugt sind, an der Erörterung und Beschlussfassung gesamtgesellschaftlich verbindlicher Entscheidungen mitzuwirken, “ und sich dadurch von ,den Beamten der öffentlichen Verwaltung, die diese Entscheidungen vorbereiten und ausführen "192, unterscheiden. So beschließen die Abgeordneten des niedersächsischen Landtags jährlich den Haushaltsplan, in dem die Finanzmittel für das NIFP bereitgestellt werden. Die Mittel finden sich im jeweiligen Einzelplan des niedersächsischen Wirtschaftsministeriums, in dem auch bestimmte Vorgaben für die Verwendung der Mittel, wie beispielsweise die allgemeinen Ziele des Programms sowie die Förderschwerpunkte, aufgeführt sind. ${ }^{193}$ Über die konkrete Verwendung der Mittel entscheiden die Mitarbeiter des Ministeriums unter Berücksichtigung der Vorgaben der NIFP-Richtlinie. Es handelt sich hierbei um eine Verwaltungsvorschrift, durch die der Wirtschaftsminister die Entscheidungen seiner Untergebenen steuert. ${ }^{194}$ Das generelle Schema einer solchen Verwaltungsvorschrift, die die Gewährung von Zuwendungen regelt, ist allerdings von der Niedersächsischen Staatskanzlei vorgegeben. ${ }^{195}$ So müssen in der Zuwendungsrichtlinie u. a. Zuwendungszweck, Förderungsgegenstand, Zuwendungsempfänger, Zuwendungsvoraussetzungen, Art, Umfang und Höhe der Zuwendung sowie das Förderungsverfahren näher ausgeführt werden. Wurde ein Richtlinienentwurf von den Mitarbeitern des Ministeriums unter Beachtung der Vorgaben des Ministers und der Staatskanzlei erstellt, muss dieser mit Verbänden, dem Finanzministerium und den übrigen Ressorts abgestimmt werden. Im Fall der NIFP-Richtlinie wurden beispielsweise die NBank und das Innovationszentrum in die Abstimmung eingebunden. Anschließend wird der Richtlinienentwurf der Staatskanzlei zur Durchsicht übersendet und der Landesrechnungshof angehört. Die endgültige Fassung der Richtlinie wird im Niedersächsischen Ministerialblatt veröffentlicht. ${ }^{196}$

Zusammenfassend stellt sich die Rolle der regionalen Politiker im NIFP folgendermaßen dar: Als Abgeordnete des Landtags und politische Führung der beteiligten Ministerien und der Staatskanzlei tragen sie die Verantwortung für die Schaffung des NIFP und geben dessen Rahmenbedingungen vor. Die konkrete Ausarbeitung des Programms sowie dessen Durchführung obliegt dann

192 Vgl. Schmidt (1995), S. 371.

193 Vgl. z. B. für das Haushaltsjahr 2008 den Einzelplan 08 - Ministerium für Wirtschaft, Arbeit und Verkehr, S. 160 ff., in: http://cdl.niedersachsen.de/blob/images/C13227050 L20.pdf, zugegriffen am 05.08.2008.

194 Vgl. Maurer (2006), § 24, Rn. 1 und 11.

195 Vgl. Niedersächsische Staatskanzlei (2006), S. 4 ff.

196 Vgl. Niedersächsische Staatskanzlei (2006), S. 16 f. 
den Bürokraten (vgl. Abschnitt 4.4). Welches Interesse Politiker an der Schaffung von Innovationsförderprogrammen wie dem NIFP haben, wird im folgendem Abschnitt untersucht.

\subsubsection{Das politische Interesse an Innovationsförderung}

Wie in Abschnitt 3.2 gezeigt, ist die traditionelle Annahme vom Politiker als wohlwollendem Planer, dessen Interesse einzig in der Steigerung des Gemeinwohls besteht, überholt. Realistischer ist die im Rahmen der Neuen Politischen Ökonomie und insbesondere in der Theorie der politischen Konjunkturzyklen verwendete Annahme, dass politische Amtsinhaber ein starkes Interesse an ihrer Wiederwahl haben. Nun stellt sich die Frage, inwiefern Wirtschaftsförderung und insbesondere Innovationsförderung zur Wiederwahl beitragen kann.

Das Modell der politischen Konjunkturzyklen (vgl. Abschnitt 3.2.1) lässt sich mit dem Themenkomplex der Innovationsförderung insofern in Zusammenhang bringen, wenn nicht das klassische Modell von Nordhaus mit Arbeitslosigkeit und Inflation als die Wähler interessierenden Variablen verwendet wird, sondern die Variable Wirtschaftswachstum ins Spiel kommt. Wenn die Wähler ein hohes Wirtschaftswachstum positiv bewerten, beurteilen sie Politiker nach der Ausprägung des Wirtschaftswachstums und treffen dementsprechend ihre Wahlentscheidung. Die Wähler geben ihre Stimme denjenigen Politikern, die ihnen das höchste Wirtschaftswachstum und somit den höchsten Nutzen versprechen. Eine dynamische Wirtschaftsentwicklung zeigt die Kompetenz der Amtsinhaber in wirtschaftlichen Fragen. Ist die Wirtschaftsentwicklung der Region dagegen schlechter als die anderer Regionen oder des Landesdurchschnitts, dann sind die Wähler unzufrieden und geben ihre Stimme der Opposition und nicht den Amtsinhabern. ${ }^{197}$

Nun entscheiden Politiker in der Realität allerdings nicht allein über die Ausprägung der volkswirtschaftlichen Variablen. Insbesondere die Beeinflussung des Wirtschaftswachstums gestaltet sich als schwierig. In Abschnitt 2.4 wurde im Rahmen der endogenen Wachstumstheorie der empirisch belegte positive Zusammenhang zwischen Innovation und Wirtschaftswachstum beschrieben, welcher allerdings nur langfristig wirkt. Die an ihrer Wiederwahl interessierten Politiker können somit mit Hilfe von Innovationsförderung das Wirtschaftswachstum in ihrer Region mit Blick auf den Wiederwahlzeitpunkt steigern. Auch wenn das Mittel der Innovationsförderung für eine kurzfristige Beeinflussung des Wirtschaftswachstums nicht geeignet ist, so stellt Innovationsförderung aus Mangel an Alternativen oder zur Ergänzung anderer Politikmaßnahmen ein sinnvolles Instrument der Politik zur Steigerung des langfristigen Wirtschaftswachstums dar. Das lässt die Schlussfolgerung zu, dass Politiker daran interessiert sind, vorrangig diejenigen Unternehmer zu fördern, die mit

Vgl. Hartig (1990), S. 114; Hansmeyer (1977), S. 985 f. 
ihren Innovationen besonders zu Wachstums- und Beschäftigungseffekten in der Region bzw. der Volkswirtschaft beitragen. ${ }^{198}$

Unterstützung für diese Argumentation lässt sich in den Wahlprogrammen der Parteien für den niedersächsischen Landtagswahlkampf 2008 finden. Die CDU möchte Niedersachsen als Innovationsstandort etablieren und das Land damit zum ,attraktiven Anlaufpunkt für Unternehmen, Wissenschaftler und Forscher ${ }^{6199}$ machen, u. a. durch eine verbesserte Mittelausstattung für die Innovationsförderung. Die SPD betont, dass gerade in forschungsintensiven Wirtschaftszweigen in der Zukunft viele Arbeitsplätze entstehen werden, und betont deswegen die Notwendigkeit einer strategischen Innovationspolitik, während FDP und Grüne ebenfalls explizit auf die Bedeutung einer Innovationspolitik und -förderung zur Stärkung des Wachstums und der Arbeitsmarktsituation Niedersachsens hinweisen. ${ }^{200}$

Neben dem langfristigen Zusammenhang zwischen Innovation und Wirtschaftswachstum gibt es noch einen zweiten Grund, warum Politiker Innovationsförderung betreiben. Durch die besondere Förderung bestimmter Unternehmen und Branchen können sie sich die Stimmen der mit diesen Unternehmen bzw. Branchen verbundenen und sympathisierenden Wähler sichern. ${ }^{201}$ Diese Argumentation basiert darauf, dass die Gesamtheit der Steuerzahler, welche für die Finanzierung der Fördermaßnahmen aufkommt, nicht bemerkt, dass sie für erhöhte Ausgaben der Förderung aufkommen müssen. Deswegen gehen den Politikern nicht die Stimmen der zahlenmäßig den Geförderten meist weit überle-

198 Diese Annahme scheint für die Mehrheit der Regionalpolitiker gültig zu sein. Eine Ausnahme bildet der Fall einer Regierung, die Innovationen bewusst unterdrückt, weil die von den Innovationen profitierenden Gruppen der Regierung gefährlich werden könnten. Somit versucht die Regierung diese Innovationen zu ersticken, damit die Innovatoren keinen ökonomischen und daraus evtl. resultierenden politischen Machtzuwachs erhalten, vgl. das Modell von Chaudhry und Garner (2007). Es lassen sich zwar einige historische Beispiele für dieses Regierungsverhalten finden, dennoch ist dieses Verhalten für demokratische Gesellschaften von wenig Belang. Für weitere historische Beispiele von innovationshemmenden Gesellschaften vgl. Landes (1999), S. $200 \mathrm{ff}$.

199 Vgl. das Regierungsprogramm der CDU für 2008-2013 „Zukunftsland Niedersachsen“, S. 28, in: http://www.cdu-niedersachsen.de/themen/regierungsprogramm/Regierungsprogramm_2008-2013.pdf, zugegriffen am 01.04.2008.

200 Vgl. das Regierungsprogramm der SPD für 2008-2013 „Gerechtigkeit kommt wieder“, S. $11 \mathrm{f}$, in: http://www.spdnds.de/imperia/md/content/landesverbandniedersachsen/ltw 2008/regierungsprogrammweb.pdf, zugegriffen am 01.04.2008, S. 28; das FDP-Programm zur Landtagswahl „Richtiges fortsetzen“, S. 6, in: http://www.fdp-nds.de/fdp nds/files/01_wahlprogramm_endgfassung_20_09_07_mit_deckblatt.pdf, zugegriffen am 01.04.2008; das Landeswahlprogramm 2008-2013 der Grünen „Jetzt. Für morgen“, S. 23, in: http://www.gruene-niedersachsen.de/cms/default/dokbin/207/207239.landtagswahlprogramm2008.pdf, zugegriffen am 01.04.2008.

201 Vgl. Hartig (1990), S. 106. 
genen Wähler verloren. ${ }^{202}$ Wird die Finanzierungslast allerdings zu hoch, dann besteht die Gefahr, dass Politiker von den Wählern für ihre Großzügigkeit bei der Ausgestaltung der Wirtschaftsförderung bestraft werden und die Wähler ihre Stimmen sparsameren Politikern geben. ${ }^{203}$

Ein dritter politökonomischer Grund für den Einsatz von Innovationsförderung als Politikmittel besteht darin, dass die Amtsinhaber mit Hilfe von Wirtschaftsförderung allgemein und Innovationsförderung im Besonderen ihre Aktivität zeigen, um die Wähler von ihrer wirtschaftspolitischen Kompetenz zu überzeugen. ${ }^{204}$ Deswegen sind Politiker daran interessiert, eine Vielzahl von Förderprogrammen zu kreieren und umzusetzen, um diese in der Öffentlichkeit und im Wahlkampf für die Erhöhung ihrer Reputation zu nutzen. ${ }^{205}$

Aufbauend auf der in Abschnitt 3.2.2 beschriebenen „rent seeking“-Theorie lässt sich ein weiterer Grund für das Interesse der Politik an Innovationsförderung finden. So könnten sich Unternehmer- und Branchenverbände für die Einführung und Stärkung von Innovationsförderprogrammen einsetzen, weil einige ihrer Mitgliedsunternehmen von diesen Programmen profitieren. Allerdings erhält nur ein verschwindend geringer Anteil der Verbandsmitglieder diese Fördermittel. Außerdem erfolgt der Auswahlprozess der Förderung durch die Einbeziehung verschiedener bürokratischer Ebenen und kann von den Interessengruppen selbst nicht direkt beeinflusst werden. Deswegen ist anzunehmen, dass Verbandsvertreter ihre Anstrengungen eher auf andere Maßnahmen konzentrieren, von denen alle oder zumindest ein großer Teil ihrer Mitglieder profitieren, wie beispielsweise Steuererleichterungen oder branchenspezifische Gesetzesvorhaben. Aus diesem Grund spielen Interessengruppen und ihre Einflussnahme im weiteren Verlauf der Arbeit keine Rolle, weswegen die Theorieansätze des „rent seeking“ nicht weiter angewendet werden.

\subsubsection{Fazit}

Zusammenfassend ist festzustellen, dass Politiker im Allgemeinen ein hohes Interesse an Innovationsförderung haben. Die Förderung soll Unternehmen darin unterstützen, Innovationsprojekte umzusetzen, die in besonderem Maße zu Wachstum und Beschäftigung in der Region bzw. Volkswirtschaft beitragen und die ohne Förderung nicht durchgeführt worden wären. Diese Feststellung wird durch die Ergebnisse der Neuen Politischen Ökonomie, insbesondere der Theorie der politischen Konjunkturzyklen, unter Berücksichtigung der Erkenntnisse

202 Vgl. Issing (1979), S. 51.

203 Vgl. Hartig (1990), S. 106; 109 f.

204 Vgl. Fölster (1991), S. 35 f. und 48 ff.

205 So weist schon Hansmeyer (1977) darauf hin, dass Transferzahlungen (Subventionen) eher vom Wähler bemerkt werden als Staatsausgaben, die der Allgemeinheit zugute kommen. 
der endogenen Wachstumstheorie unterstützt. Obwohl Politiker keine das Gemeinwohl maximierenden sozialen Planer sind, haben sie aufgrund ihres Eigeninteresses, wiedergewählt zu werden, den Anreiz, genau diejenigen Unternehmer zu fördern, die in Abschnitt 2.4 im Rahmen der wachstumspolitischen Implikationen beschrieben wurden.

Hypothese 3: Politiker haben den Anreiz, relativ sichere Innovationsprojekte risikoscheuer Unternehmer zu fördern, die erfahren in der Innovationstätigkeit sind, was genau der Zielgruppe der wachstumspolitischen Begründung für Innovationsförderung entspricht.

Diese Tatsache wird auch anhand der Formulierung der Ziele des NIFP in der Förderrichtlinie deutlich, wie in Abschnitt 2.4 erläutert wurde. Die Politiker bestimmen zwar die Leitlinien der niedersächsischen Innovationspolitik, indem sie die erforderlichen Haushaltsmittel bereitstellen und als politische Führung der Staatskanzlei und Ministerien die entsprechenden Richtlinien verantworten. Allerdings kümmern sich die zuständigen Bürokraten um die konkrete Durchsetzung der Förderprogramme und die Mittelvergabe. Den Beamten des Referats Industrie- und Technologiepolitik des niedersächsischen Wirtschaftsministeriums obliegt die Entscheidung über die Förderanträge unter Berücksichtigung der Antragsevaluationen des Innovationszentrums, der NBank und den Innovationsberatern der Handwerkskammern.

Aufgrund der wichtigen Position der Bürokraten in der institutionellen Ausgestaltung des NIFP beschäftigt sich der folgende Abschnitt ausführlich mit dieser Gruppe, die die Fördermittel verwaltet. Dabei geht es um die Fragestellungen, inwiefern die Anreize und Verhaltensweisen der Bürokraten mit denjenigen der Politiker bezogen auf Innovationsförderung übereinstimmen und ob Interessenkonflikte auftreten. Des Weiteren wird untersucht, welche Konsequenzen die Verteilung der Macht auf die unterschiedlichen Akteursgruppen auf die Umsetzung des NIFP hat.

\subsection{Die Rolle der Bürokratie}

Im Sinne der Theorie der Bürokratie können die Beamten des zuständigen Referats des Wirtschaftsministeriums als Bürokraten bezeichnet werden. Wie in Abschnitt 4.2 dargestellt, entscheiden sie über die Fördermittelvergabe. Allerdings treffen sie diese Entscheidung nicht allein, sondern auf Basis der Evaluationen anderer Institutionen. Die NBank ist hierbei für die formale bzw. wirtschaftliche Evaluation verantwortlich, während die technologische Evaluation vom Innovationszentrum Niedersachsen für den F\&E-Programmteil und von den Innovati- 
onsberatern der Handwerkskammern für den Handwerksprogrammteil vorgenommen wird.

Das Innovationszentrum Niedersachsen ist als $\mathrm{GmbH}$ organisiert, allerdings ist das Land Niedersachsen, vertreten durch die Landesregierung, hundertprozentiger Gesellschafter des Innovationszentrums und gleichzeitig einziger Auftraggeber. Das Innovationszentrum bezeichnet sich selbst auf seiner Homepage als „unabhängiger Berater und Think Tank für die niedersächsische Landesregierung ${ }^{\text {“206. }}$. Zu seinen Aufgaben gehören neben Technologiebeobachtung und -bewertung die Konzeption, Beratung und Begleitung von technologiepolitischen Maßnahmen, wobei das Innovationszentrum nur insofern in die Programmumsetzung eingebunden ist, als dass seine Mitarbeiter die technologische Förderwürdigkeitsprüfung für Projektskizzen und Anträge des Innovationsförderprogramms vornehmen. Neben der Evaluation von Instrumenten der Innovationsförderung ist das Innovationszentrum außerdem für die Umsetzung eines technologieorientierten Standortmarketings und die Betreuung des Innovationsnetzwerks Niedersachsen, welches mehr als 200 niedersächsische Institutionen der Innovations- und Wirtschaftsförderung umfasst, zuständig. Die Mitarbeiter des Innovationszentrums sind keine Beamten sondern Angestellte, aber da sie de facto ausschließlich für die Landesregierung und die Ministerien arbeiten und ihre Organisation nicht auf die Erzielung von Gewinnen ausgerichtet ist, können sie als Bürokraten im Sinne Niskanens (vgl. Abschnitt 3.3.1) bezeichnet werden.

Dieselbe Argumentation ist auf die Innovationsberater der Handwerkskammern anwendbar. Handwerkskammern sind Körperschaften des öffentlichen Rechts und vertreten die Interessen der einer Pflichtmitgliedschaft unterliegenden Handwerksunternehmen in einem bestimmten Kammerbezirk $(\S \S 90,91$ $\mathrm{HwO}^{207}$ ). Als Selbstverwaltungskörperschaften des Handwerks gehören sie zur mittelbaren Staatsverwaltung ${ }^{208}$ und unterstehen der Staatsaufsicht des zuständigen Wirtschaftsministeriums ( $§ 115$ Abs. 1 HwO). Die Organisation der Handwerkskammern ist nicht auf die Erzielung von Gewinnen hin ausgerichtet und erzeugt mit den ihr übertragenen hoheitlichen Aufgaben einen nicht marktfähigen Output. Diese Anhaltspunkte sprechen dafür, die Handwerkskammern als Bürokratien zu bezeichnen. Die Rolle der Kammern als politische Interessenvertretung des Handwerks spricht allerdings gegen die Eigenschaft einer Bürokratie, so dass die Handwerkskammern nur teilweise als Bürokratie angesehen werden können.

206 Internetseite des Innovationszentrums, in: http://www.iz-nds.de, zugegriffen am 29.03. 2008.

207 Handwerksordnung in der Fassung der Bekanntmachung vom 24.09.1998 (BGBl. I S. 3074; 2006 I S. 2095), zuletzt geändert durch Art. 9a des Gesetzes vom 07.09.2007 (BGBl. I S. 2246).

208 Vgl. Maurer (2006), § 23, Rn. 31. 
In jeder niedersächsischen Handwerkskammer gibt es einen Innovationsberater, der die Unternehmen des Kammerbezirks bei der Entwicklung und Vermarktung innovativer Produkte und neuer Technologien unterstützen soll. $\mathrm{Zu}$ seinen konkreten Aufgaben gehört sowohl die Hilfestellung bei der Finanzierung von Innovationsprojekten, wozu auch die Beratung in Bezug auf in Frage kommende Innovationsförderprogramme zählt, als auch die Beratung bei Fragen der Patentierung, Zertifizierung und der Vermarktung. Außerdem ist der Innovationsberater für Fragen des Technologietransfers und der Zusammenarbeit mit den Hochschulen zuständig. ${ }^{209}$ Die Aufgaben der Innovationsberater zählen zum Bereich des nicht marktgängigen Outputs der Handwerkskammern, so dass die Innovationsberater als Bürokraten bezeichnet werden können.

Die NBank ist als universale Förderbank der Ansprechpartner in Niedersachsen für alle Förderprogramme der Europäischen Union, des Bundes, des Landes und der Kommunen in den Bereichen Wirtschafts-, Arbeitsmarkt-, Wohnraum- und Infrastrukturförderung. ${ }^{210}$ Die NBank gehört zu $100 \%$ dem Land Niedersachsen, vertreten durch die Landesregierung. Auch die Mitarbeiter der NBank können somit als Bürokraten bezeichnet werden, da die NBank vollständig im öffentlichen Besitz ist und mit der Verwaltung der Fördermittel einen nicht marktgängigen Output produziert. ${ }^{211}$

Im Folgenden werden die in Abschnitt 3.3 vorgestellten Bürokratietheorien auf die Anreize und das Verhalten der am NIFP beteiligten Akteure angewendet. Die aufgezeigten Anreizstrukturen und daraus resultierenden Verhaltensweisen können das Verhalten der Akteure in der Realität nicht vollständig erklären. Doch können mit Hilfe dieser Analyse wertvolle Erkenntnisse geliefert werden, die vorliegende Interessenkonflikte zwischen offiziellen Zielen der Innovationsförderung und inoffiziellen Zielen der beteiligten Institutionen betreffen. Diese Interessenkonflikte können dazu führen, dass manche Entscheidungsträger nicht

$209 \mathrm{Vgl}$. beispielsweise die Informationen zum Innovationsberater auf der Internetseite der Handwerkskammer Hildesheim-Südniedersachsen, in: http://www.hwk-hildesheim.de/ 24, index.html, zugegriffen am 26.03.2008.

210 Vgl. die Internetseite der NBank, in: http://www.nbank.de/Die_NBank/index.php, zugegriffen am 30.03. 2008.

211 Bis Ende des Jahres 2007 gehörte die NBank zu $50 \%$ dem Land Niedersachsen, vertreten durch die Landesregierung, und zu $50 \%$ der Nord-LB. Die Nord-LB gehört wiederum zu 41,75\% dem Land Niedersachsen, vertreten durch die Hannoversche Beteiligungsgesellschaft mbH, zu 32,79\% dem Sparkassenverband Niedersachsen, zu 8,25\% dem Land Sachsen-Anhalt, zu 7,53 \% dem Sparkassenbeteiligungsverband Sachsen-Anhalt und zu 5,22 \% dem Sparkassenbeteiligungszweckverband Mecklenburg-Vorpommern, vgl. Nord/LB, Geschäftsbericht 2007, S. 44, in: https://www.nordlb.de/filead$\mathrm{min} /$ Investor_Relations/Geschaeftsberichte/deutsch/2007/Geschaeftsbericht_2007.pdf, zugegriffen am 02.08.2008. Somit war die NBank auch in der Vergangenheit vollständig in öffentlichem Besitz und konnte als Bürokratie angesehen werden. 
im Sinne der offiziellen, der eigentlichen Ziele der Innovationsförderung handeln.

\subsubsection{Die Einordnung der Bürokratiegruppen in den Ablauf des NIFP}

In diesem Abschnitt wird die Einbindung der verschiedenen Bürokratengruppen in den Innovationsförderprozess untersucht. Abbildung 10 stellt den Ablauf der Prüfung der Projektskizze für den F\&E-Programmteil dar. Zunächst bewertet das Innovationszentrum die technologische Förderwürdigkeit der Projektskizzen und somit der Anträge mit Hilfe einer Technologieanalyse, einer Markteinschätzung und einer Ressourcenbewertung. ${ }^{212}$ In der Technologieanalyse werden u. a. der Innovationsgrad und die Konkurrenzsituation auf nationaler Ebene untersucht. Die Technologieanalyse wird von einem Mitarbeiter des Innovationszentrums selbst durchgeführt und eventuell durch eine Analyse der fachlich zuständigen Landesinitiative ergänzt, wie z. B. den Landesinitiativen für Brennstoffzellen, Telematik oder Life Sciences. ${ }^{213}$ Die Markteinschätzung analysiert die potentiellen Kundengruppen auf Bedürfnisse, Kundennutzen und Zielbranchen, den Entwicklungsstand des Marktes und die Markttrends. In der Ressourcenbewertung werden sowohl die schon im Unternehmen vorhandenen bzw. für das Innovationsprojekt aufzubauenden Personalressourcen betrachtet als auch die Wissensressourcen, die intern vorhanden sind oder extern über Netzwerke, Kunden, Zulieferer und Forschungseinrichtungen beschafft werden können.

Eine sehr wichtige Rolle bei der Bewertung spielen auch die technologische Strahlwirkung und das Wertschöpfungspotential des Innovationsprojekts. Überregionale Kooperationen können gefördert werden, doch muss der Großteil der Wertschöpfung und des Wissenszuwachses in Niedersachsen verbleiben. Unter Berücksichtigung grundlegender Charakteristika des Unternehmens werden das technische und das wirtschaftliche Risiko überprüft. Nur bei Vorhandensein beider Risiken wird eine Förderempfehlung ausgesprochen. Denn falls ein Unternehmen das Innovationsprojekt selber finanzieren kann, da es bei-

212 Vgl. „Bewertung von FuE-Vorhaben im Innovationsförderprogramm Niedersachsen“ auf der Internetseite des Innovationszentrums, in: http://www.iz-nds.de/downloads/ Nortrup211105.pdf, zugegriffen am 29.03.2008. Die folgenden Absätze zur Prüfung der Förderanträge durch das Innovationszentrum basieren außerdem auf dem Gespräch mit Herrn Dr. Buß vom 28.03.2008 in Anhang B.

213 Die Landesinitiativen haben die Aufgabe, themenspezifische Netzwerke für bestimmte Zukunftstechnologien über technologische Trends und Fördermöglichkeiten zu informieren, Kooperationen und Innovationsprojekte anzuregen sowie Öffentlichkeitsarbeit und Standortmarketing für Niedersachsen zu betreiben. $\mathrm{Zu}$ diesem Zweck werden Landesinitiativen vom Wirtschaftsministerium eingerichtet und zunächst für drei Jahre finanziert, bevor nach einer Evaluation durch das Innovationszentrum Niedersachsen über eine Anschlussfinanzierung entschieden wird, vgl. http://www.innovatives.niedersachsen.de/870.html, zugegriffen am 31.03.2008. 
spielsweise über ausreichende Gewinne verfügt oder weil die Technologie vollständig ausgereift ist, besteht keine Notwendigkeit zur Förderung.

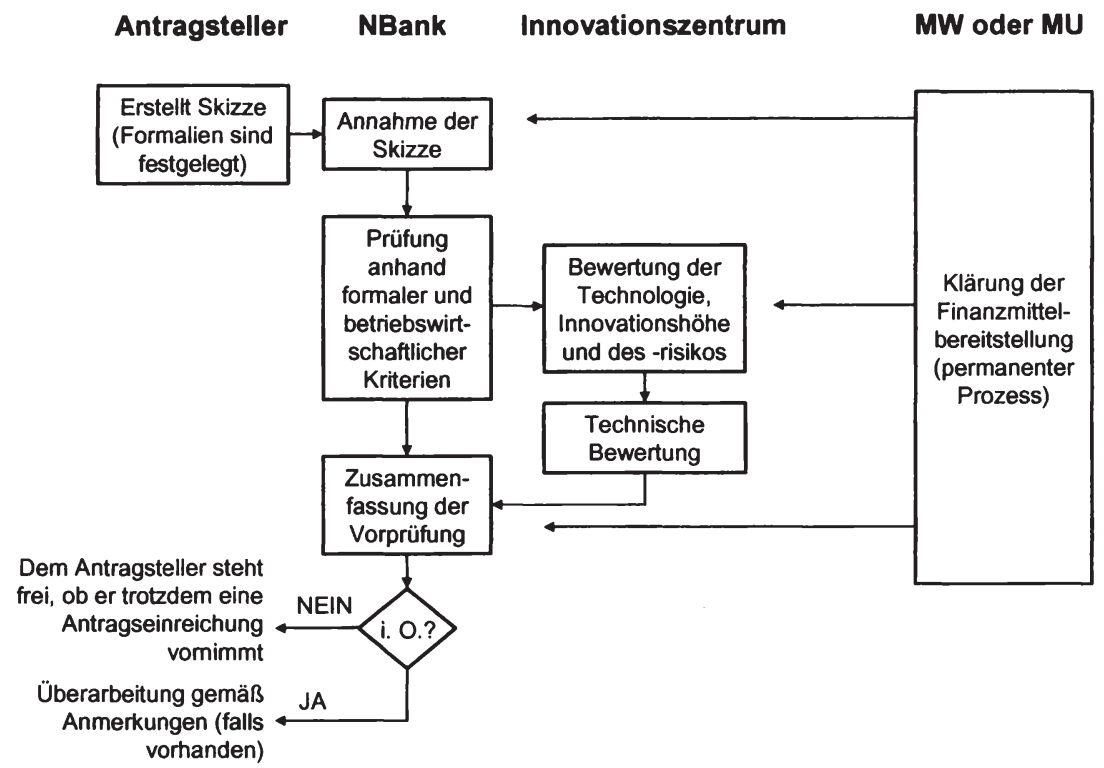

Abbildung 10: Ablauf der Skizzenprüfung (Eigene Darstellung nach Innovationszentrum Niedersachsen ${ }^{214}$ ).

Die Förderung im Handwerksprogrammteil basiert auf den Förderempfehlungen der Innovationsberater der Handwerkskammern. Ebenso wie das Innovationszentrum nehmen die Innovationsberater neben der technologischen und organisatorischen Bewertung auch die Bewertung der unternehmerischen Finanzsituation vor. Dabei ist es wichtig, dass die Finanzsituation der Unternehmen so gut ist, dass die Finanzierung ihres Anteils gesichert ist. Das technologische Risiko ist im Regelfall beherrschbar. Die Vermarktung stellt die Unternehmen dagegen vor erhebliche Schwierigkeiten. ${ }^{215}$ Technologisches und wirtschaftliches Risiko scheinen also in diesem Programmteil eine eher untergeordnete Rolle zu spielen. Bei der Bewertung der Risiken ist meist das Marktrisiko entscheidend.

Die Innovationsberater der Handwerkskammern sind sehr viel stärker als die Mitarbeiter des Innovationszentrums schon vor Fertigstellung der Projekt-

214 Vgl. „Bewertung von FuE-Vorhaben im Innovationsförderprogramm Niedersachsen“ auf der Internetseite des Innovationszentrums, Folie 7, in: http://www.iz-nds.de/downloads/Nortrup211105.pdf, zugegriffen am 29.03. 2008.

215 Vgl. Gespräch mit Herrn Strunk-Lissowski am 27.03.2008 in Anhang A. 
skizze in den Prozess der Antragstellung eingebunden. Handwerksunternehmer, die durch Meldungen in Lokalzeitungen, mittels Verbandsmitteilungen, Informationsveranstaltungen der Handwerkskammern oder aber durch die Vermittlung der Kreishandwerkerschaften auf das Innovationsförderprogramm aufmerksam werden, wenden sich an den zuständigen Innovationsberater, der mit ihnen die betriebsbezogenen Chancen und Risiken des Projektes diskutiert. Der Innovationsberater unterstützt die interessierten Unternehmen weiterhin bei der Erstellung der Projektskizze und des Projektantrags und berät sie in Hinblick auf eine erfolgreiche Antragstellung. ${ }^{216}$

Wichtige Kriterien für die Bewertung des Projektantrags durch die Innovationsberater bestehen darin, dass die Innovation auf Bedürfnisse der Nachfrager eingeht und somit Erfolgschancen am Markt besitzt. Außerdem muss der Stand der Technik ausführlich dargestellt sein, was mit Hilfe einer Patent- und Technikrecherche erfolgen kann. Auch die Finanzierung des durch das Unternehmen zu finanzierenden Eigenanteils muss gesichert sein. Der Innovationsberater macht sich bei Unternehmensbesuchen selbst ein Bild von der Unternehmensorganisation und den im Unternehmen vorhandenen Ressourcen und beurteilt nach seinen Kenntnissen, inwiefern das Unternehmen geeignet erscheint, das beantragte Innovationsprojekt auch tatsächlich durchzuführen. ${ }^{217}$

Die NBank übernimmt die Prüfung der Antragskizze anhand formaler und betriebswirtschaftlicher Kriterien. In der Praxis bedeutet das, dass die NBank überprüft, ob das Unternehmen in der Lage ist, seinen Eigenanteil am Innovationsprojekt zu finanzieren. Die schwierige Bewertung der Frage, ob das Unternehmen auch ohne Förderung in der Lage gewesen wäre, das Innovationsprojekt durchzuführen, erfolgt nicht.

\subsubsection{Prinzipal-Agent-Beziehung zwischen Politik und Bürokratie}

Die Beziehung zwischen Politikern und Bürokraten in einem Innovationsfördersystem kann mit Hilfe der Prinzipal-Agent-Theorie analysiert werden. Die Politiker sind die Prinzipale, die die Förderprogramme entwerfen und beschließen, während die Bürokraten als Agenten die Programme ausführen. Es bestehen Informationsasymmetrien zwischen den beiden Akteursgruppen im Sinne von „hidden action“ (vgl. Abschnitt 3.1.3), weil die Politiker die Tätigkeit der Bürokraten nicht vollständig überwachen können.

Bezogen auf Niedersachsen besteht die Macht der Beamten des Wirtschaftsministeriums darin, dass sie erstens bei der Ausgestaltung der Förderprogramme mitarbeiten und dass ihnen zweitens - unter Berücksichtigung der Evaluationen von NBank, Innovationszentrum und Innovationsberatern der Handwerkskammern - die Entscheidung darüber obliegt, welche Unternehmen För-

216 Vgl. Gespräch mit Herrn Strunk-Lissowski am 27.03.2008 in Anhang A.

217 Vgl. Gespräch mit Herrn Strunk-Lissowski am 27.03.2008 in Anhang A. 
derung erhalten. Die Politiker wissen also nicht, ob die Bürokraten im Ministerium auch tatsächlich die Unternehmen fördern, für die sie die Innovationsförderprogramme entworfen haben, nämlich diejenigen Unternehmen, die für Wirtschaftswachstum und Beschäftigungszuwächse in der Volkswirtschaft sorgen (vgl. Abschnitt 4.3). ${ }^{218}$ Die Ministerialbeamten sind keine reinen Erfüllungsgehilfen der Politiker, sondern stellen eigenständige Entscheidungseinheiten dar, deren Wirken die Effizienz der Innovationsförderung entscheidend mit beeinflusst.

Wie in jeder Prinzipal-Agent-Beziehung ist es für die Politiker schwierig, eine Erfolgskontrolle der Bürokraten vorzunehmen aufgrund der beschriebenen Bewertungsprobleme des Bürokratieoutputs (vgl. Abschnitt 3.3.1). Bezogen auf Innovationsförderung ist eine genaue Überwachung der Tätigkeit der Bürokraten deswegen schwierig, weil die Politiker sich aus Mangel an Zeit und Erfahrung nicht mit den Details der Fördermittelanträge und Innovationsprojekte beschäftigen können. Deswegen müssen sie die Outputkontrolle anhand anderer, einfach messbarer Indikatoren vornehmen (vgl. Abschnitt 3.3.4). ${ }^{219}$

Zudem ist aber auch eine detaillierte statistische Erfolgsmessung eines Innovationsförderprogramms mit verschiedenen Problemen behaftet. Um die Entwicklung geförderter Unternehmen zu beurteilen, wird ihre Entwicklung mit derjenigen einer Vergleichsgruppe gegenübergestellt, die aus nicht geförderten Unternehmen besteht. Dieser Vergleich ist allerdings aus drei Gründen problematisch und mit möglichen Fehlern behaftet. Erstens erfolgt die Auswahl der Fördermittelempfänger nicht zufällig im Sinne eines Quasi-Experiments, sondern hängt von nicht beobachtbaren Unternehmenscharakteristika ab, so dass ein „selection bias" vorliegt. ${ }^{220}$ Zweitens treten bei Existenz von externen Effekten durch Innovation, die die hauptsächliche Rechtfertigung für die Förderprogramme darstellen (vgl. Abschnitt 2.3.1), Schwierigkeiten auf. Wenn positive externe Effekte von geförderten auf nicht geförderte Unternehmen auftreten, wird die Wirkung der Förderung auf die geförderten Unternehmen unterschätzt. Deswegen konstatieren Klette et al. (2000), ,if an evaluation study finds little difference between the supported firms and the non-supported firms it could either be because the $R \& D$ program was unsuccessful and generated little innovation, or because the $R \& D$ program was highly successful in generating new innovations which created large positive spillovers to the non-supported firms “ $" 221$ Drittens müssten in eine vollständige Evaluation auch externe Effekte der

218 Gläser (2002), S. $110 \mathrm{ff}$., kommt in seiner Analyse der Prinzipal-Agent-Beziehungen bezogen auf Existenzgründungsförderung zu einem ähnlichen Schluss für das Bundeswirtschaftsministerium als wirtschaftspolitische Entscheidungsinstanz.

219 Vgl. Wallsten (2000), S. $84 \mathrm{f}$.

220 Vgl. Klette et al. (2000), S. 472 und S. 478 f.

221 Klette et al. (2000), S. 482. 
Förderung auf Konsumentenrenten einbezogen werden, welche aber nicht quantifizierbar sind (vgl. Abschnitt 2.4).

In Niedersachsen ist für Zuwendungsrichtlinien eine Erfolgskontrolle vorgesehen, um die Notwendigkeit der Zuwendung zu überprüfen, eventuelle Änderungen vorzunehmen und eine Verlängerung des zeitlich begrenzten Zuwendungsprogramms zu begründen. ${ }^{222}$ Bislang erfolgte eine Evaluation des Innovationsförderprogramms nur durch die NBank im Rahmen einer Betrachtung der Verwertungsberichte, die die geförderten Unternehmen zwei Jahre nach der Förderung abgeben müssen, um über den Markterfolg des geförderten Produktes, die geschaffenen Arbeitsplätze und weitere Erfolgsindikatoren zu berichten. Da das Programm in dieser Form erst seit Mitte 2004 existiert, liegen noch nicht viele Verwertungsberichte vor. ${ }^{223}$

Den Bürokraten des Wirtschaftsministeriums ist als Budgetmaximierern (vgl. Abschnitt 3.3.1) stark daran gelegen, das Programm gegenüber den Politikern als erfolgreich darzustellen, um das Programm auch zukünftig zu verwalten und dabei über ähnliche oder erweiterte Budgets zu verfügen. Infolgedessen besteht ein Anreiz für die Bürokraten darin, die Auswahl der zu fördernden Projekte eher risikoscheu vorzunehmen (vgl. Abschnitt 3.3.3), um eine hohe Erfolgsquote der geförderten Projekte vorzuweisen. Im Zweifelsfall tendieren Bürokraten dazu, die Innovationsprojekte mit einer geringen Ausfallwahrscheinlichkeit zu fördern, weil sie gegenüber den Politikern in Erklärungsnot geraten, wenn geförderte Projekte scheitern bzw. geförderte Unternehmer trotz Förderung aus dem Markt ausscheiden oder keine Innovationen hervorbringen. ${ }^{224}$ Es besteht kaum Anreiz für die Bürokraten, besonders riskante, aber im Erfolgsfall für die Volkswirtschaft stark lohnende Projekte zu fördern, die aus Gründen des allokativen Marktversagens (vgl. Abschnitt 2.3.3) keine Finanzierung über den Markt erhalten.

Wenn die ministerialen Bürokraten vorzugsweise relativ sichere Innovationsprojekte fördern, können sie außerdem Erfolgsbeispiele geförderter Unternehmen vorweisen, eventuell verbunden mit Umsatz- und Beschäftigungszuwächsen. Diese Unternehmen können wiederum auf Veranstaltungen als Musterbeispiele für eine erfolgreiche Innovationspolitik (auch „Leuchttürme“ genannt) vermarktet werden, wovon wiederum die Politiker in ihrem Wiederwahlbestreben profitieren (vgl. Abschnitt 3.2.1). ${ }^{225}$

222 Vgl. Niedersächsische Staatskanzlei (2006), S. 16.

223 Die Angaben wurden der Verfasserin von der NBank übermittelt.

224 Vgl. Lerner (1999), S. 292 ff.; Lach (2002), S. 371; vgl. außerdem Gläser (2002), S. 112 ff. und 120 ff., mit einer ähnlichen Argumentation bezogen auf Ausgleichs- und Geschäftsbanken im Bereich der Existenzgründungsförderung.

225 Vgl. Lerner (1999), S. 296 f.; Wallsten (2000), S. 85. Zur Leuchtturmpolitik allgemein vgl. Steinrücken und Jaenichen (2003). 


\subsubsection{Spezifische Interessen der verschiedenen Bürokratengruppen}

Wie in Abschnitt 3.3 erläutert wurde, sind Bürokraten nach der Theorie von Niskanen an einer vollständigen Ausschöpfung der ihnen zur Verfügung stehenden Geldmittel interessiert, um ihr Budget in zukünftigen Perioden auszuweiten und dadurch mehr Macht und Prestige zu erlangen. ${ }^{226}$ Somit erfolgt nicht unbedingt eine Verwendung der Fördermittel durch die Bürokraten ausschließlich für nach Meinung der Politiker sinnvolle Projekte. Stattdessen spielen die Eigeninteressen der Bürokraten eine wesentliche Rolle. Denn Bürokraten haben einen Anreiz nach dem „first come, first serve“-Prinzip zu verfahren und die erstbesten Projekte zu fördern, die ihren Zielvorgaben entsprechen, um ihr Budget auszuschöpfen. In ihrem Verhalten entsprechen sie somit der Theorie des „satisficing" (vgl. Abschnitt 3.1.1), d. h. sie unterstützen nicht die besten Projekte, sondern die ersten Projekte, auf die sie stoßen, die ihren Erwartungen genügen. Die institutionelle Ausgestaltung des Programms, das keine festen Bewerbungstermine im F\&E-Programmteil vorsieht, unterstützt dieses Verhalten. Ministerialbeamte würden also dieser Theorie folgend dem Anreiz unterliegen, möglichst viele der ihnen vorgelegten Förderanträge zu genehmigen, selbst wenn Mitnahmeeffekte (vgl. Abschnitt 4.5.1.2) wirksam werden.

Die institutionelle Ausgestaltung des NIFP wirkt diesem möglichen Anreizmechanismus entgegen. Denn die Bewertung der Fördermittelanträge liegt nicht vollständig in der Macht der Ministerialbeamten. Die Förderanträge werden zunächst von NBank, Innovationszentrum bzw. den Innovationsberatern der Handwerkskammern auf technologische und wirtschaftliche Förderfähigkeit evaluiert. Diese Förderempfehlungen beeinflussen die Entscheidungsfindung der Bürokraten in erheblicher Weise. Laut Aussagen der beteiligten Akteure folgen die Ministerialbeamten in der Regel den Förderempfehlungen und weichen nur in Ausnahmefällen von diesen ab.

Die Mitarbeiter des Innovationszentrums haben gegenüber den Ministerialbeamten einen Informationsvorsprung durch technologische Kompetenz. Ihnen obliegt neben der strategischen Aufgabe der Politikberatung bei der Erstellung der Förderprogramme auch die operative Aufgabe der Bewertung der Anträge des F\&E-Programmteils. Durch die Einbindung des Innovationszentrums versuchen die Ministerialbeamten ihren Informationsnachteil gegenüber den Unternehmern zu verringern.

Die Mitarbeiter des Innovationszentrums haben aus zwei Gründen ein gewisses Interesse daran, dass das Programm erfolgreich ist und von Politik und Ministerium positiv bewertet wird. Erstens muss der Aufsichtsrat des Innovationszentrums, der zurzeit aus vier Staatssekretären und einer Regierungsdirekto-

226 Vgl. Hartig (1990), S. 108 f. 
rin besteht, ${ }^{227}$ mit ihrer Arbeit zufrieden sein. Zweitens erhöht ein erfolgreiches Programm die Reputation der verantwortlichen Mitarbeiter und des Innovationszentrums insgesamt. Um den Erfolg des Programms zu gewährleisten, haben die Mitarbeiter des Innovationszentrums den Anreiz, die Projektskizzen bzw. Förderanträge derjenigen Unternehmen positiv zu evaluieren, die zu Wachstumsund Beschäftigungseffekten für Niedersachsen beitragen oder die als technologische „Leuchttürme“ dienen können. Zu diesem Zweck dienen auch die Evaluationskriterien der technologischen Strahlwirkung und des regionalen Wertschöpfungspotentials. ${ }^{228}$ Im Falle eines schlecht laufenden Programms würde die Kompetenz des Innovationszentrums ebenfalls gefragt, um neue technologiepolitische Maßnahmen zu entwerfen und zu begleiten. Deswegen haben die Mitarbeiter keinen Anreiz, den Programmerfolg übermäßig positiv darzustellen.

Da das Budget des Innovationszentrums festgelegt ist und nicht vom Budget des Förderprogramms abhängt, haben die Mitarbeiter keinen Anreiz, möglichst viele Förderanträge positiv zu bewerten. In ihren Evaluationen werden explizit die technischen und wirtschaftlichen Risiken der Innovationsprojekte überprüft. Beide Risikoarten müssen gegeben sein, damit ein Projekt eine positive Förderempfehlung erhält. ${ }^{229}$ Zusammenfassend ist davon auszugehen, dass die Evaluationen des Innovationszentrums im Sinne der Politiker (vgl. Abschnitt 4.3.3) erstellt werden.

Die Innovationsberater der Handwerkskammern haben ebenso wie die Mitarbeiter des Innovationszentrums aus Reputationsgründen ein gewisses Interesse daran, dass das Programm erfolgreich ist und von Politik und Ministerialbeamten positiv bewertet wird. Deswegen haben auch sie den Anreiz, die Förderanträge derjenigen Unternehmen positiv zu evaluieren, die zu Wachstums- und Beschäftigungseffekten beitragen oder die als technologische „Leuchttürme“ dienen können. Die Erfolgsgeschichten von geförderten Unternehmen können sowohl andere Unternehmen zu einer verstärkten Innovationstätigkeit anhalten als auch gleichzeitig als Werbung für das Förderprogramm und für die Region Niedersachsen dienen. Aus Engagement für ihren Kammerbezirk, der durch Wachstums- und Beschäftigungseffekte von einer möglichen Förderung profitiert und durch technologische „Leuchttürme“ stärker als Technologiestandort wahrgenommen wird, haben sie den zusätzlichen Anreiz, eher Unternehmen aus ihrem Kammerbezirk für das Programm vorzuschlagen und durchzusetzen. Es ist anzunehmen, dass alle Innovationsberater diesem Anreiz unterliegen und dass die in Abschnitt 4.2 beschriebene Runde aus den Innovationsberatern und

227 Vgl. die Internetseite des Innovationszentrums Niedersachsen, in: http://www.iz-nds.de/ ansprechpartner.php, zugegriffen am 30.03.2008.

228 Vgl. „Bewertung von FuE-Vorhaben im Innovationsförderprogramm Niedersachsen“ auf der Internetseite des Innovationszentrums, in: http://www.iz-nds.de/downloads/ Nortrup211105.pdf, zugegriffen am 29.03.2008.

229 Vgl. Gespräch mit Herrn Dr. Buß vom 28.03.2008 in Anhang B. 
Vertretern anderer Institutionen dafür sorgt, dass eine gleichmäßige regionale Streuung der Förderempfehlungen innerhalb Niedersachsens erfolgt. ${ }^{230} \mathrm{Die} \mathrm{Be}-$ rücksichtigung regionaler Interessen deutet allerdings darauf hin, dass die Gefahr besteht, nicht immer die Projekte mit der höchsten Förderwürdigkeit auszuwählen.

Da die NBank als Förderbank die Fördermittel auszahlt und verwaltet, ist davon auszugehen, dass ihre Mitarbeiter aus Budgetmaximierungsgründen an einer Ausweitung der Förderprogramme interessiert sind. Da es sich in der Praxis nicht um eine Darlehensförderung handelt, besteht für die NBank auch kein Risiko durch Gläubigerausfall. So unterliegen die Mitarbeiter der NBank dem Anreiz, möglichst viele Förderanträge positiv zu bewerten und das Förderbudget so schnell wie möglich auszuschöpfen. Allerdings beschränkt sich der Evaluationsanteil der NBank darauf, ob die Förderanträge den formalen Anforderungen genügen, so dass der Einfluss der NBank bei der Auswahl der zu fördernden Unternehmen als eher gering eingestuft werden kann. Eine zu schnelle Mittelausschöpfung ist laut Aussage aller Beteiligten nicht gegeben. Bei mangelnder Mittelverfügbarkeit werden besonders interessante Projekte zurückgestellt, um ihnen zu einem späteren Termin Mittel zukommen zu lassen, sobald wieder Fördermittel zur Verfügung stehen. Es kann auch eine Mittelverschiebung auf ministerialer Ebene erfolgen. Diese Argumentation gilt jedoch offenbar nur für den F\&E-Programmteil. Im Handwerksprogrammteil stehen aufgrund der Ende 2007 erlassenen Zuwendungsbescheide für den gesamten Zeitraum des Jahres 2008 keine Mittel mehr zur Verfügung (vgl. Abschnitt 4.2).

\subsubsection{Fazit}

Da alle betrachteten Bürokratengruppen von der ihnen zugewiesenen Finanzausstattung durch die Politiker abhängig sind, haben sie den Anreiz, ein erfolgreiches Innovationsförderprogramm vorzuweisen. Deswegen sind ihre Interessen mit denen der Politiker fast identisch. Bürokraten besitzen aber des Weiteren einen Anreiz, eher risikoärmere Innovationsprojekte zu fördern, die die Erfolgsbilanz des Förderprogramms verbessern:

Hypothese 4: Die verschiedenen Gruppen der Bürokratie haben den Anreiz, eher sichere Innovationsprojekte risikoscheuer Unternehmer zu fördern, die über ausreichend Erfahrung die Innovationstätigkeit betreffend verfügen.

$230 \mathrm{Vgl}$. für eine ähnliche Argumentation in Bezug auf das „Small Business Innovation Research Program“ in den USA Lerner (1999), S. 296. 


\subsection{Die Unternehmer als Fördermittelempfänger des NIFP}

Die letzten Abschnitte haben die Gemeinsamkeiten und Unterschiede der Interessen der beteiligten Akteure in Politik und Bürokratie im Rahmen der Institution Innovationsförderung aufgezeigt. Bislang wurden aber diejenigen Akteure noch nicht analysiert, für die die Innovationsförderprogramme aufgelegt werden, nämlich die Unternehmer, die Fördermittel beantragen und erhalten. Dieser Abschnitt bezieht deswegen die Gruppe der Unternehmer in die institutionenökonomische Analyse der Innovationsförderung mit ein und beschäftigt sich zunächst mit der Prinzipal-Agent-Beziehung zwischen Bürokraten und Unternehmern und den daraus resultierenden Interessenkonflikten und Anreizsituationen (Abschnitt 4.5.1). Die Grenzen der institutionenökonomischen Analyse werden anschließend in Abschnitt 4.5.2 aufgezeigt.

\subsubsection{Prinzipal-Agent-Beziehung zwischen Bürokratie und Unternehmern}

Neben der Prinzipal-Agent-Beziehung zwischen Politikern und Bürokraten liegt noch eine weitere Prinzipal-Agent-Beziehung in der institutionellen Ausgestaltung des NIFP vor: Die Bürokraten als Fördermittelgeber sind die Prinzipale und die Unternehmer als Fördermittelempfänger die Agenten. Wie in jeder Beziehung zwischen Finanzier und finanziertem Unternehmer verfügen letztere über bessere Informationen hinsichtlich ihrer Förderprojekte und ihres Unternehmens als die Prinzipale, wobei verschiedene Informationsasymmetrien vorliegen. Zum einen bestehen Informationsasymmetrien im Sinne von "hidden information“ (vgl. Abschnitt 3.1.3), weil die Bürokraten nicht so gut über die Projektqualität informiert sind wie die Unternehmer selbst (Abschnitt 4.5.1.1). Zum anderen kann „hidden action“ (vgl. Abschnitt 3.1.3) vorliegen, weil die Bürokraten nicht überwachen können, ob die Unternehmer die erhaltenen Fördermittel wirklich für die angegebenen Projekte einsetzen (Abschnitt 4.5.1.2).

\subsubsection{Erste Informationsasymmetrie: die Auswahl der zu fördernden Pro- jekte}

Die beschriebene erste Informationsasymmetrie („hidden information") liegt in der Projektauswahl begründet. Die bürokratischen Entscheidungsträger wählen die zu fördernden Projekte aus und stehen vor der Schwierigkeit, dass sie systematisch schlechter über die Qualität der Projekte und die Beschaffenheit des unternehmerischen Managements informiert sind als die Antrag stellenden Unternehmer. Eine mögliche Lösung besteht im „signalling“, d. h. der Unternehmer verfolgt bestimmte Verhaltensweisen, um dem Finanzier seine Qualität bzw. die 
Qualität des Projektes zu beweisen. ${ }^{231}$ So könnte die unternehmerische Innovationstätigkeit der Vergangenheit den Bürokraten als Signal für die Förderfähigkeit des Unternehmens dienen. ${ }^{232}$

Eine weitere Lösung der Informationsasymmetrie besteht im „screening“, d. h. die bürokratischen Entscheidungsträger sammeln Informationen über den Unternehmer und das zu fördernde Projekt, um eine fundierte Förderentscheidung zu treffen. Zur Bestimmung des Qualitätsgehalts eines Innovationsvorhabens kann es sinnvoll sein, die fachliche Expertise einer unabhängigen externen Institution zu Rate zu ziehen. Für den F\&E-Programmteil des Förderprogramms nimmt das Innovationszentrum die technologische Bewertung vor, während die NBank die finanzielle Bewertung durchführt. Da die geförderten Projekte i. d. R. mit hoch komplexen technologischen Sachverhalten aus den verschiedensten Branchen zu tun haben und sich das Innovationszentrum auf Technologieberatung spezialisiert hat, erscheint diese Lösung sinnvoll. Die Qualitätskontrolle der beantragten Projekte durch das Innovationszentrum soll gewährleisten, dass nur besonders innovative Projekte durch das NIFP gefördert werden. Es ist anzunehmen, dass derartige Projekte hauptsächlich von generell innovativen Unternehmen durchgeführt werden, die auch schon in der Vergangenheit eine starke F\&E- bzw. Innovationsaktivität aufwiesen. Das ergibt sich zum einen aus dem kumulativen Charakter von F\&E bzw. Innovation. Zum anderen haben generell innovativ tätige Unternehmen höchstwahrscheinlich relativ oft innovative Projektideen, mit denen sie sich erfolgreich um Fördermittel bewerben können.

\section{Hypothese 5: Geförderte Unternehmen weisen eine höhere Innovati- onstätigkeit auf als nicht geförderte Unternehmen.}

\subsubsection{Zweite Informationsasymmetrie: „rent seeking“ und Mitnahme- effekte}

Die zweite Informationsasymmetrie zwischen Bürokraten und Unternehmern (,hidden action") beschäftigt sich mit dem Problem, dass der Unternehmer als Agent nach Vertragsschluss, also nach Eingang der Förderbeziehung, die Fördermittel nicht im Sinne des Prinzipals nutzt, sondern seinen eigenen, vom Nutzen des Prinzipals abweichenden Nutzen maximiert. Die Fördermittel stellen für die Unternehmer kein Fremdkapital dar, sondern bilden einen Zuschuss zum Eigenkapital, da die Mittel nicht zurückgezahlt werden müssen. Es besteht die Ge-

231 Vgl. Goodacre und Tonks (1995), S. 304, für eine allgemeine Untersuchung der Informationsasymmetrien zwischen Finanzier und Finanziertem bei der Finanzierung von F\&E-Projekten.

232 Vgl. Wallsten (2000), S. 94 f. 
fahr des „moral hazard“. ${ }^{233}$ Der Prinzipal erwartet eine sinnvolle Verwendung seiner Mittel, wie im vorliegenden Fall eine verstärkte Innovationstätigkeit des Unternehmens. Nun kann der Unternehmer die Fördermittel unter falschen Versprechungen erlangen. Er kann genau die vom Prinzipal gewünschten Effekte versprechen, aber von vornherein nicht die Absicht haben, die Mittel für diese Zwecke auch einzusetzen, was einer Form des „rent seeking“ entspricht (vgl. Abschnitt 3.2.2 zur Anwendung dieses Begriffs im Bereich der Politik). ${ }^{234}$ Beim NIFP ist der Unternehmer allerdings dazu verpflichtet, einen detaillierten Verwendungsnachweis über die Ausgabe der Fördermittel sowie Zwischenberichte und einen Endbericht darüber abzugeben, inwiefern die angestrebten Ziele auch tatsächlich erreicht wurden. „Rent seeking“ wird somit durch das „reporting“ der Unternehmer und das ,monitoring“ der NBank weitgehend unterbunden, da der Unternehmer die Fördermittel zurückzahlen muss, wenn er das beantragte Projekt nicht durchführt.

Neben dem ,rent seeking“ besteht allerdings eine weitere Möglichkeit, dass der Unternehmer die Fördermittel nicht im Sinne des Prinzipals nutzt, nämlich wenn der Unternehmer die Mittel auch ohne Förderung hätte aufbringen können und auch tatsächlich für das geförderte Projekt eingesetzt hätte. Dieses Phänomen wird als Mitnahmeeffekt bezeichnet und wird in der Literatur ausführlich behandelt. ${ }^{235}$ Neben der Bestimmung des Innovationsgehalts der Projekte müssen die Entscheidungsträger der Verwaltung eine Bewertung vornehmen, welche Innovationsprojekte das Unternehmen auch ohne Fördermittel durchführen würde. Diese Projekte sollen explizit nicht gefördert werden, weil hier Mitnahmeeffekte vorliegen. Denn dadurch dass Unternehmer Fördermittel erhalten und für die Innovationsprojekte einsetzen, werden andere Mittel frei, die für andere Zwecke innerhalb des Unternehmens eingesetzt werden können. Die Mitnahmeeffekte können auch partiell auftreten, d. h. dass ein Unternehmen einen Teil des Innovationsprojektes auch ohne Förderung durchgeführt hätte, aber durch die Förderung in die Lage versetzt wird, das Projekt in einem größeren Ausmaß umzusetzen.

233 Vgl. Arrow (1962b), S. 613, und Goodacre und Tonks (1995), S. 308 ff., für eine ähnliche Argumentation in Bezug auf die Finanzierung von F\&E-Projekten.

234 Eine grundlegend andere Situation entsteht übrigens, wenn der Unternehmer die Fördermittel in Form eines zinsgünstigen Darlehens bekommt, welches er nach einer gewissen Zeit zurückzahlen muss. Hier handelt es sich um Fremdkapital, bei dem das übliche „moral hazard“-Problem zwischen Finanzier und Finanziertem auftritt, weil der Unternehmer das Fremdkapital anders behandelt und investiert als Eigenkapital, vgl. Arrow (1962b), S. 612 f. Wenn der Unternehmer das Fremdkapital aber wie in der Literatur allgemein angenommen risikoreicher investiert als das Eigenkapital, so kann das in Bezug auf F\&E/Innovation sogar wünschenswert sein, weil die mit F\&E verbundenen Risiken und positiven externen Effekte ja dazu führen, dass in der gesamten Volkswirtschaft zu wenig F\&E bereitgestellt wird (vgl. Abschnitt 2.3).

235 Vgl. Zimmermann (1987) und David et al. (2000). 
Allerdings bestehen erhebliche Schwierigkeiten in der empirischen Überprüfbarkeit von Mitnahmeeffekten, da die reale Situation (mit Förderung) mit einer hypothetischen Referenzsituation (ohne Förderung) verglichen wird. Dieses Problem tritt nicht auf, wenn die Förderung während des betrachteten Zeitraums eingeführt wurde. Denn dann kann ein Zeitraum, in dem es noch keine Förderung gab, mit einem Zeitraum mit Förderung verglichen werden, was als Policy-off-/Policy-on-Ansatz bezeichnet wird. Doch diese Möglichkeit besteht nur in den seltensten Fällen. Deswegen müssen alternative Methoden zur Erhebung von Primärdaten verwendet werden, wie beispielsweise Befragungen von Fördermittelempfängern oder soziale Experimente, in denen eine Teilgruppe der Teilnehmer fiktive Fördermittel erhält. ${ }^{236}$ Bei Befragungen der geförderten Unternehmer besteht allerdings die Schwierigkeit, dass diese nicht wahrheitsgemäß auf die Frage antworten, ob sie das Innovationsprojekt auch ohne Fördermittel durchgeführt hätten. Denn ihnen ist bewusst, dass ihre Antwort Einfluss auf die zukünftige Ausgestaltung von Förderprogrammen hat. ${ }^{237}$ In der Literatur herrschen aufgrund dieser Problematik Regressionsschätzungen auf Basis von Zeitreihen oder Querschnitten vor, die geförderte und nicht geförderte Unternehmen miteinander vergleichen. Ein häufig verwendeter Ansatz ist auch das Matchingverfahren, d. h. ein Vergleich von Referenzpaaren, die sich nur in Bezug auf den Tatbestand der Förderung unterscheiden, aber deren sonstige Charakteristika möglichst identisch sind. ${ }^{238}$

Die Ergebnisse der empirischen Studien sind bezogen auf die quantitative Bedeutung von Mitnahmeeffekten und der daraus resultierenden Relevanz für die Wirtschaftspolitik nicht einheitlich. Es gibt Studien, die eine vollständige Substitutionalität feststellen, also ein vollständiges „crowding out" der privaten F\&E-Ausgaben in Höhe der entsprechenden öffentlichen Fördermittel. Andere Studien finden keinerlei Anzeichen für eine irgendwie geartete Substitutionalität. ${ }^{239}$ Einen ausführlichen Überblick über Studien zum Thema Mitnahmeeffekte findet sich bei David et al. (2000). Von 33 empirischen Studien aus dem Zeitraum von 1966 bis 1999 kommen elf zu dem Ergebnis, dass öffentliche F\&EAusgaben (teilweise in Form von Fördermitteln, teilweise allgemeine F\&EAusgaben des Staates) als Substitut für private F\&E-Ausgaben dienen. ${ }^{240}$ Wallsten (2000) findet in seiner Untersuchung der 1990-1992 durch das „Small Business Innovation Research Program" geförderten US-Unternehmen deutliche Hinweise auf ein „crowding out“ der privaten F\&E-Ausgaben durch die Fördermittel und kann sogar eine vollständige Substitutionalität nicht ausschlie-

236 Vgl. Bizer et al. (2003), S. 61.

237 Vgl. Wallsten (2000), S. 86, Fn. 10; Fier (2002), S. 173; Fölster (1991), S. 33.

238 Vgl. zum Matchingverfahren Czarnitzki und Fier (2002), S. 6 ff.; Fier et al. (2005), S. 7 ff.; Fier (2002), S. 254 ff.

239 Vgl. David et al. (2000), S. 511, Fn. 25; Scott (2000), S. 65 f.

240 Vgl. David et al. (2000), S. 526. 
Ben. ${ }^{241}$ Die meisten anderen Untersuchungen kommen allerdings zu anderen Ergebnissen. Czarnitzki und Fier (2002) zeigen bei ihrer Untersuchung des deutschen Dienstleistungssektors, dass die Innovationsintensität der geförderten Unternehmen signifikant höher ist als die der nicht geförderten Unternehmen, so dass sie eine vollständige Substitutionalität der Fördermittel ausschließen können. Da sie keine Daten bzgl. der Höhe der Fördermittel haben, können sie aber keine quantitative Aussage über die Höhe etwaiger Mitnahmeeffekte treffen. ${ }^{242}$ Für ostdeutsche Unternehmen des Verarbeitenden Gewerbes in den Jahren 1996 und 1998 erhält Czarnitzki (2001) dasselbe Resultat. ${ }^{243}$ Lach (2002) stellt für israelische Unternehmen des Verarbeitenden Gewerbes im Zeitraum von 19901995 fest, dass die Förderung zu einer deutlichen Erhöhung der privaten F\&EAusgaben führte. Diese Erhöhung ist allerdings geringer als die im Rahmen des Förderprogramms aufzubringenden Eigenanteile, so dass die Untersuchung auf die Existenz von Mitnahmeeffekten schließen lässt. ${ }^{244}$ Auch Görg und Strobl (2007) finden Hinweise darauf, dass F\&E-Förderung in Irland im Zeitraum 1999-2002 zu einer Erhöhung der unternehmerischen F\&E-Tätigkeit geführt hat. Bei großen Fördersummen scheinen allerdings wiederum Mitnahmeeffekte vorzuliegen. ${ }^{245}$

Die meisten empirischen Studien kommen folglich zu dem Ergebnis, dass es teilweise Mitnahmeeffekte bei F\&E- und Innovationsförderprogrammen gibt, aber dass keine vollständige Substitutionalität erfolgt, so dass die Förderprogramme zu zusätzlichen Investitionen der Unternehmen führen. Bei der Interpretation von diesen Ergebnissen muss außerdem Folgendes berücksichtigt werden: Erstens wird ein F\&E- bzw. Innovationsprojekt durch die Förderung früher durchgeführt, als es dem Unternehmen ohne Fördermittel möglich gewesen wäre. ${ }^{246}$ Zweitens ist es möglich, dass Unternehmen durch die Förderung inhaltlich andere Projekte durchführen, d. h. Projekte mit höheren externen Grenzerträgen, die von den Unternehmen aufgrund der mangelnden Aneignungsmöglichkeiten dieser Grenzerträge ohne Förderung nicht durchgeführt worden wären (vgl. Abschnitt 2.3.1) ${ }^{247}$ Drittens kann Innovationsförderung dazu führen, dass ein Projekt durch das Unternehmen anders durchgeführt wird, in dem Sinne, dass das Unternehmen durch die Antragstellung sein Projektmanagement verbessert. So können insbesondere Handwerksunternehmen, die bislang keine ausgeprägte F\&E-Tätigkeit aufweisen, die Strukturierung von F\&E-Prozessen durch die

241 Vgl. Wallsten (2000), S. $96 \mathrm{f}$.

242 Vgl. Czarnitzki und Fier (2002), S. $10 \mathrm{ff}$.

243 Vgl. Czarnitzki (2001), S. 15.

244 Vgl. Lach (2002), S. $384 \mathrm{ff}$.

245 Vgl. Görg und Strobl (2007), S. 231.

246 Vgl. Bizer et al. (2003), S. 62; Scott (2000), S. 65 f.

247 Vgl. Scott (2000), S. 65 f.; Busom (2000), S. 114. 
Einbindung in das NIFP/Handwerk lernen - ein Effekt, der nicht unmittelbar durch das Programm intendiert ist, aber dennoch in der Realität relevant ist. ${ }^{248}$

Zusammenfassend sind Mitnahmeeffekte bei jeglicher Form von Wirtschaftsförderung nicht zu vermeiden. Da sie empirisch schwer nachzuweisen sind, muss die entsprechende Evidenz kritisch betrachtet werden. Insbesondere der Vergleich zwischen geförderten Unternehmen und einer Kontrollgruppe nicht geförderter Unternehmen ist mit Schwierigkeiten behaftet, weil die Zuordnung von Unternehmen zu den beiden Gruppen nicht zufällig erfolgt. ${ }^{249} \mathrm{Be}-$ stimmte Charakteristika sind nicht nur dafür verantwortlich, dass sich Unternehmen für Fördermittel bewerben und ihre Förderanträge positiv bewertet werden, sondern beeinflussen auch die weitere Unternehmensentwicklung. Diese Arbeit hat es sich zum Ziel gesetzt, auf die Spur einiger dieser Charakteristika zu kommen.

Bislang ging es in dieser Arbeit um die Zielsetzung der Innovationsförderung und den Auswahlprozess der zu fördernden Unternehmer im Rahmen des niedersächsischen Programms. Doch nicht alle Unternehmen, die für ein Innovationsförderprogramm in Frage kommen, bewerben sich um Fördermittel. Der folgende Abschnitt beschäftigt sich mit diesem Thema, das deswegen entscheidend für die wirtschaftspolitische Bewertung jeglicher Innovationsförderprogramme ist, weil die Auswahl der zu fördernden Unternehmer nur aus der Gruppe der Bewerber getroffen werden kann, die auf das Programm aufmerksam werden und den Bewerbungsaufwand auf sich nehmen.

\subsubsection{Die Wahrnehmung von Förderangeboten}

Neben den in den Abschnitten 4.5.1.1 und 4.5.1.2 dargestellten Informationsasymmetrien gibt es eine weitere Asymmetrie zwischen Bürokraten und Unternehmern, die in der Literatur bislang weitgehend unbeachtet blieb. Hier stellen nun die Bürokraten die besser informierte Partei dar, da die Informationsasymmetrie in der vielen Unternehmen unübersichtlich erscheinenden Förderlandschaft, dem so genannten „Förderdschungel“, begründet liegt. Kognitive Grenzen und die „bounded rationality“ (vgl. Abschnitt 3.1.1) spielen hier eine wesentliche Rolle in der Wahrnehmung von Förderangeboten durch die Unternehmer. Denn allein für niedersächsische Unternehmen gibt es zurzeit 128 Förderprogramme bzw. Programmteile der Fördermittelgeber Land, Bund und EU für die inhaltlichen Bereiche F\&E und Innovation. Für ganz Deutschland sind es sogar 262 Programme bzw. Programmteile. ${ }^{250}$ Bei dieser Fülle an Förderprogrammen und darüber verfügbaren Informationen muss der einzelne Unterneh-

248 Vgl. für diese Einschätzung bezogen auf das NIFP das Gespräch mit Herrn StrunkLissowski vom 27.03. 2008 in Anhang A.

249 Vgl. Wallsten (2000), S. 88: Görg und Strobl (2007), S. 220 f.; Busom (2000), S. 114.

$250 \mathrm{Vgl}$. http://www.foerderdatenbank.de, zugegriffen am 13.02.2008. 
mer erhebliche Transaktionskosten (vgl. Abschnitt 3.1.2) darauf verwenden, um die für sein Unternehmen relevanten Fördermöglichkeiten zu entdecken und sich mit der konkreten Ausgestaltung der Förderprogramme und den jeweiligen Konditionen zu beschäftigen.

Deswegen ist anzunehmen, dass sich ein erheblicher Teil der eigentlich förderwürdigen Unternehmen nicht um Förderung bemüht. Insbesondere kleine Unternehmen haben nicht die personellen, zeitlichen und finanziellen Ressourcen, sich zunächst über Fördermöglichkeiten zu informieren und sich anschlieBend für ein Förderprogramm zu bewerben. Diese Schlussfolgerung lässt drei Möglichkeiten offen: Erstens könnte diese Entwicklung politisch nicht gewollt sein, sich aber durch die institutionelle Ausgestaltung des Fördersystems ergeben haben. Zweitens besteht die Möglichkeit, dass die Unübersichtlichkeit politisch gewollt ist. Diejenigen Unternehmen, die es schaffen, die unübersichtliche Förderlandschaft zu durchdringen, signalisieren dem Fördermittelgeber damit ihre Qualität (vgl. zum ,signalling“ Abschnitt 4.5.1.1), so dass der Förderdschungel als Differenzierungsmechanismus dient. Drittens könnte das Bestreben der Politiker, den Wählern Aktivität zu signalisieren, die Vielfalt des Förderprogrammangebots verschuldet haben.

\subsubsection{Fazit}

Bevor es im Rahmen des NIFP zu einer Förderung kommt, liegt eine Informationsasymmetrie zwischen Bürokraten und potentiell $\mathrm{zu}$ fördernden Unternehmern vor. Die Unternehmer müssen teilweise erhebliche Transaktionskosten aufwenden, um die unübersichtliche „Förderlandschaft" nach für sie in Frage kommenden Förderprogrammen zu durchsuchen. Diese Informationsasymmetrie wird bislang in der Literatur weitgehend vernachlässigt - im Gegensatz zur Prinzipal-Agent-Beziehung zwischen den Bürokraten als Fördermittelgebern und Unternehmern als Fördermittelempfängern.

In dieser Prinzipal-Agent-Beziehung liegen zwei Informationsasymmetrien vor. Die Erste beschreibt das Problem, dass die Unternehmer über bessere Informationen bezüglich ihrer Innovationsprojekte verfügen als die Bürokraten. Um diese Informationsasymmetrie zu überwinden, existiert eine Qualitätskontrolle der beantragten Projekte durch das Innovationszentrum und die Innovationsberater der Handwerkskammern. Es ist anzunehmen, dass die Qualitätskontrolle dafür sorgt, dass nur besonders innovative Projekte durch das NIFP gefördert werden. Derartige Projekte werden hauptsächlich von Unternehmen durchgeführt, die in Vergangenheit und Gegenwart eine starke F\&E- bzw. Innovationsaktivität aufweisen. Dafür spricht zum einen der kumulative Charakter von F\&E bzw. Innovation. Zum anderen haben generell innovativ tätige Unternehmen im Vergleich zu bislang nicht innovativ tätigen Unternehmen wahrscheinlich eher sehr innovative Projektideen, die für die Bewerbung um Innovations- 
fördermittel geeignet sind. Deswegen wurde die Hypothese aufgestellt, dass geförderte Unternehmen eine höhere Innovationstätigkeit aufweisen als nicht geförderte Unternehmen (Hypothese 5).

Die zweite Informationsasymmetrie beschäftigt sich mit dem Problem der Nutzung der Fördermittel durch den Agenten, also den Unternehmer, für Zwecke, die nicht im Sinne des Prinzipals, also des Bürokraten, sind. Theoretisch möglich sind hier ,rent seeking“ und Mitnahmeeffekte. Jedoch wird „rent seeking“ durch das vom Förderprogramm verlangte Reporting und das „monitoring" der NBank weitgehend unterbunden. Was die Mitnahmeeffekte anbetrifft, so können diese nicht vollständig ausgeschlossen werden.

\subsection{Zusammenfassung der Ergebnisse und Hypothesen}

In diesem Kapitel wurde die institutionelle Ausgestaltung des NIFP mit Hilfe der Theorieansätze der Neuen Institutionenökonomik untersucht. Um die Fragestellung zu beantworten, welche Unternehmen und Unternehmer tatsächlich gefördert werden, wurde zunächst der europarechtliche Rahmen für Wirtschaftsförderung im Allgemeinen und für Innovationsförderung im Besonderen dargestellt, der die Vorgaben für jegliches politische Handeln liefert. Grundsätzlich herrscht in der EU ein präventives Beihilfeverbot mit Erlaubnisvorbehalt. Ein Innovationsförderprogramm kann als staatliche Beihilfe entweder aufgrund einer Genehmigung der Europäischen Kommission oder einer Gruppenfreistellungsordnung gestattet werden. Das NIFP/F\&E wurde als Ermessensausnahme von der Europäischen Kommission zugelassen, während das NIFP/Handwerk unter die Gruppenfreistellungsordnung für „De-minimis-Beihilfen“ fällt.

An die europarechtliche Darstellung anschließend wurden die Konsequenzen der Machtverteilung auf verschiedene Akteursgruppen für das NIFP betrachtet. $\mathrm{Zu}$ diesem Zweck wurden die Prinzipal-Agenten-Beziehungen zwischen den verschiedenen beteiligten Akteursgruppen betrachtet und dabei die speziellen Interessen und Verhaltensweisen der unterschiedlichen Akteursgruppen in die Analyse mit einbezogen.

Unter Berücksichtigung der Erkenntnisse der Neuen Politischen Ökonomie haben Politiker ein hohes Interesse an Innovationsförderung. Sie wollen durch die Förderung Unternehmer darin unterstützen, Innovationsprojekte umzusetzen, welche diese ohne Förderung nicht durchgeführt hätten. Diese Feststellung wird durch die Erkenntnisse der Neuen Politischen Ökonomie, insbesondere der Theorie der politischen Konjunkturzyklen, unter Berücksichtigung der Erkenntnisse der endogenen Wachstumstheorie unterstützt. Obwohl Politiker keine das Gemeinwohl maximierenden sozialen Planer sind, haben sie aufgrund ihres Wiederwahlinteresses den Anreiz, diejenigen Unternehmer zu fördern, die in besonderem Maße zu Wachstum und Beschäftigung in der Region bzw. Volkswirt- 
schaft beitragen. Diese Zielgruppe deckt sich mit der in Abschnitt 2.4 beschriebenen Zielgruppe der wachstumspolitischen Ausrichtung der Innovationsförderung. Deswegen wird folgende Hypothese aufgestellt:

\begin{abstract}
Hypothese 3: Politiker haben den Anreiz, relativ sichere Innovationsprojekte risikoscheuer Unternehmer zu fördern, die erfahren in der Innovationstätigkeit sind, was genau der Zielgruppe der wachstumspolitischen Begründung für Innovationsförderung entspricht.
\end{abstract}

Diese Hypothese wird auch durch die Formulierung der Ziele des NIFP in der Förderrichtlinie unterstützt.

Die niedersächsischen Politiker bestimmen zwar die Leitlinien der niedersächsischen Innovationspolitik, beschließen die entsprechenden Zuwendungsrichtlinien und stellen die erforderlichen Haushaltsmittel bereit. Doch um die Durchsetzung der Förderprogramme kümmern sich die zuständigen Bürokraten. Es besteht also eine Prinzipal-Agent-Beziehung zwischen den Politikern als Prinzipalen, die die Förderprogramme entwerfen und beschließen, und den Bürokraten als Agenten, die die konkrete Durchsetzung der Programme vornehmen. Da die Politiker die Tätigkeit der Bürokraten nicht vollständig überwachen können, liegt eine Informationsasymmetrie im Sinne von „hidden action“ vor. Deswegen wurde untersucht, inwiefern die Anreize und Verhaltensweisen der verschiedenen Bürokratengruppen mit denjenigen der Politiker übereinstimmen und ob Interessenkonflikte auftreten.

Den Beamten des Referats Industrie- und Technologiepolitik des niedersächsischen Wirtschaftsministeriums obliegt die Entscheidung über die Förderanträge unter Berücksichtigung der Antragsevaluationen des Innovationszentrums Niedersachsen, der NBank und den Innovationsberatern der Handwerkskammern. Da alle Bürokratiegruppen von der ihnen zugewiesenen Finanzausstattung durch die Politik abhängig sind, haben sie den mehr oder weniger stark ausgeprägten Anreiz, ein erfolgreiches Innovationsförderprogramm vorzuweisen. Deswegen sind die von ihnen geförderten Unternehmen der Zielgruppe der Politiker und somit der Zielgruppe einer wachstumspolitisch motivierten Innovationsförderung sehr ähnlich. Um die Erfolgsbilanz des Förderprogramms zu verbessern, besitzen aber insbesondere die Ministerialbeamten den Anreiz, eher risikoarme Innovationsprojekte zu fördern. Denn diese Projekte verlaufen mit einer hohen Wahrscheinlichkeit erfolgreich und können als „Leuchttürme“ der regionalen Innovationspolitik vermarktet werden. Die Ministerialbeamten folgen allerdings in aller Regel den Förderempfehlungen der anderen beteiligten Akteure und nutzen ihre Entscheidungskompetenz nur in Einzelfällen zum Widerspruch. Das Innovationszentrum hat im Vergleich zu den Ministerialbeamten einen geringeren Anreiz für eine risikoscheue Projektauswahl. Zwar profitiert auch die Reputation des Innovationszentrums von einem erfolgreichen Förder- 
programm, doch wäre die Fachkompetenz der Mitarbeiter auch bei einer Verbesserung bzw. Erneuerung des Programms von der Politik gefordert. Die Innovationsberater der Handwerkskammern profitieren dagegen besonders von technologischen „Leuchttürmen“ in ihrer Region und unterliegen deswegen dem Anreiz eine risikoaverse Projektauswahl gemäß der wachstumspolitischen Begründung von Innovationsförderung vorzunehmen. Die NBank hat nur ein relativ geringes Mitspracherecht bei den Förderentscheidungen, da sie ausschließlich die formale Förderfähigkeit untersucht. Die Ergebnisse münden in:

\section{Hypothese 4: Die verschiedenen Gruppen der Bürokratie haben den Anreiz, eher sichere Innovationsprojekte risikoscheuer Unternehmer zu fördern, die über ausreichend Erfahrung die Innovationstätigkeit betreffend verfügen.}

Doch es existiert nicht nur eine Prinzipal-Agent-Beziehung zwischen Politikern und Bürokraten. Auch zwischen Bürokraten und Unternehmern liegt eine Prinzipal-Agent-Beziehung vor, in der die Bürokraten die Prinzipale darstellen, die Fördermittel vergeben. Die Unternehmer sind demgegenüber die Agenten, die die Fördermittel erhalten und damit Innovationsprojekte durchführen. Die Prinzipale sind systematisch schlechter informiert als die Agenten, was die Qualität der Projekte an sich als auch die Verwendung der Fördermittel betrifft. Um die erste Informationsasymmetrie zu beheben, existiert im Rahmen des NIFP eine Qualitätskontrolle der beantragten Projekte durch das Innovationszentrum. Diese Qualitätskontrolle stellt sicher, dass nur besonders innovative Projekte durch das Programm gefördert werden. Derartige Projekte und Projektideen werden höchstwahrscheinlich eher von Unternehmen durchgeführt, die generell eine starke F\&E-Aktivität aufweisen und schon in der Vergangenheit innovativ tätig waren. Deswegen wird vermutet:

\section{Hypothese 5: Geförderte Unternehmen weisen eine höhere Innovati- onstätigkeit auf als nicht geförderte Unternehmen.}

Die institutionelle Ausgestaltung des NIFP verhindert ein „rent seeking“ der Unternehmen in dem Sinne, dass die Fördermittel für andere Zwecke verwendet werden. Mitnahmeeffekte können nicht vollständig ausgeschlossen werden. Allerdings tritt bei der empirischen Überprüfung von Mitnahmeeffekten häufig das Problem auf, dass die Entwicklung geförderter und nicht geförderter Unternehmen verglichen wird. Dabei wird nicht berücksichtigt, dass sich geförderte und nicht geförderte Unternehmen nicht nur im Erhalt der Fördermittel unterscheiden, sondern auch in Bezug auf andere Charakteristika.

Es drängt sich die Frage auf, welche Gruppe von Unternehmen bzw. Unternehmern durch Innovationsförderprogramme wie das NIFP tatsächlich er- 
reicht wird. Die Wahrnehmung von Förderangeboten, kognitive Grenzen und das Zurechtfinden im „Förderdschungel“" spielen hier eine wichtige Rolle. Doch können die bisher genannten Theorieansätze innerhalb der Neuen Institutionenökonomik diese Frage nicht abschließend beantworten und erklären, warum sich manche Unternehmer für Innovationsförderung bewerben und andere nicht. Deswegen muss ein weiterer theoretischer Ansatz in die institutionenökonomische Analyse mit einbezogen werden. Zu diesem Zweck verwendet diese Arbeit Entrepreneurship-Theorien, die sich mit dem Wesen des Wirtschaftssubjekts des Unternehmer beschäftigen. Mit dieser Methodik ist es möglich, zwischen bestimmten Gruppen von Unternehmern zu unterscheiden und verschiedene Verhaltensweisen bestimmter Gruppen zu erklären. Im Folgenden werden in Kapitel 5 die für diese Arbeit relevanten Entrepreneurship-Theorieansätze vorgestellt und direkt auf die Problematik der Innovationsförderung angewendet. Anschließend wird in Kapitel 6 empirisch untersucht, inwiefern EntrepreneurshipTheorien einen Beitrag zur Erklärung des Unternehmerverhaltens im Rahmen des NIFP leisten können. 


\title{
5 Die Entrepreneurship-Theorie und ihre Anwendung auf Innovationsförderung
}

\author{
"The theoretical firm is entrepreneurless - the prince of Denmark has been ex- \\ punged from the discussion of Hamlet. "251
}

Wie in Abschnitt 2.6 dargelegt, besteht aus wachstumspolitischer Sicht die Zielgruppe von Innovationsförderung aus risikoscheuen Unternehmern, die einerseits Erfahrungen in der Innovationstätigkeit mitbringen und andererseits relativ sichere Innovationsprojekte verfolgen. Im vorigen Kapitel wurde gezeigt, inwiefern Politiker und Bürokraten ein Interesse daran haben, diese Zielgruppe auch tatsächlich zu fördern und in welcher Hinsicht die institutionelle Ausgestaltung des NIFP letztlich dazu führt, dass diese Zielgruppe tatsächlich für die Förderung ausgewählt werden kann. Doch die entscheidende Frage, ob diese Unternehmer von Innovationsförderprogrammen überhaupt erreicht werden und sich dann auch bewerben, kann mit Hilfe der bislang ausgeführten institutionenökonomischen Theorieansätze nicht beantwortet werden. Im Einzelnen stellt sich die Frage, ob sich diese Unternehmer für Innovationsförderprogramme bewerben oder lieber auf den Zeit- und Kostenaufwand verzichten, den eine Bewerbung mit sich bringt und sich stattdessen andere Kapitalquellen suchen. Es besteht die Möglichkeit, dass sich nur solche innovativen Unternehmer bewerben, deren Bewerbungsaufwand aufgrund einer bürokratischen Veranlagung oder entsprechender Erfahrungen in der Vergangenheit geringer ausfällt, wie ein häufig geäußerter Vorwurf besagt. ${ }^{252}$

Diese Arbeit verwendet das Instrumentarium der Entrepreneurship-Theorie, um die Motive und Handlungen derjenigen Unternehmer zu untersuchen, die vor der Entscheidung stehen, ob sie sich für ein Innovationsförderprogramm bewerben sollen. Dieses Kapitel ist folgendermaßen aufgebaut: Nach einem kurzen Abriss über die Rolle des Unternehmers in den volkswirtschaftlichen Paradigmen in Abschnitt 5.1 erfolgt die genaue Begriffsklärung und erste Abgrenzung des Unternehmers vom „Entrepreneur“ in Abschnitt 5.2. Unter Verwendung des Bezugssystems der unternehmerischen Gelegenheiten (,entrepreneurial opportunities") von Shane und Venkataraman (2000), das in Abschnitt 5.3 näher erläutert wird, werden die verschiedenen Entrepreneurship-Theoriestränge in den Abschnitten 5.4, 5.5 und 5.6 thematisch in dieses System eingeordnet. Nach der Erläuterung dieser Theorien folgt ihre Anwendung auf die Innovationstätigkeit und die Bewerbung von Unternehmern um Innovationsfördermittel. Auf dieser

251 Baumol (1968), S. 66.

252 Vgl. Zeppernick (1985), S. 79. 
Grundlage werden Hypothesen gebildet, die anschließend in Kapitel 6 in einer empirischen Analyse eingehend untersucht werden.

\subsection{Der Unternehmer in der volkswirtschaftlichen Theorie - die Standardparadigmen}

Der Unternehmer und seine Persönlichkeit spielten in der Volkswirtschaftslehre lange keine herausragende Rolle. Das Wirtschaftssubjekt des Unternehmers wurde nur am Rande erwähnt und nicht gesondert in die ökonomischen Betrachtungen integriert. So vertraute die überwiegende Mehrheit der Klassiker auf Smiths ,invisible hand“, die die Einzelentscheidungen der Anbieter und Nachfrager auf dem Markt koordiniert und für effiziente Preise und Gütermengen sorgt. Der Unternehmer ist ein Marktteilnehmer wie jeder andere und reagiert automatisch auf die Signale des Marktes, ohne dass er dazu besondere Fähigkeiten benötigt. Smith (1776) unterscheidet nur zwischen den Funktionen des Kapitalisten gegenüber denen des Managers, wie seine Ausführungen über den Kapitalgewinn (,profits of stock") deutlich machen: „The profits of stock, it may perhaps be thought, are only a different name for the wages of a particular sort of labour, the labour of inspection and direction. They are, however, altogether different, are regulated by quite different principles, and bear no proportion to the quantity, the hardship, or the ingenuity of this supposed labour of inspection and direction. They are regulated altogether by the value of the stock employed, and are greater or smaller in proportion to the extent of this stock. "253 Smith geht zwar auf die Möglichkeit ein, dass Kapitalbesitzer und Manager nicht dieselbe Person darstellen, doch erwähnt er nicht die besondere Rolle des Unternehmers als Entscheidungsträger und die Fähigkeiten, die dieser besitzen muss, um erfolgreich tätig zu sein. ${ }^{254}$ Von Thünen (1875) kritisiert daher zu Recht: „Adam Smith, und mit ihm die mehrsten englischen Schriftsteller werfen die Zinsen des verwandten Kapitals mit dem Unternehmergewinn unter der Benennung ,Gewinn 'zusammen. "255 Unter den Klassikern stellen Say und Mill Ausnahmen dar, da sie explizit auf die herausgehobene Funktion des Unternehmers eingehen, wie in den Abschnitten 5.2.1 und 5.5.1 ausgeführt wird.

Eine plausible Erklärung für die beschriebene Vermengung von Unternehmer und Kapitalist bei den Klassikern besteht darin, dass eine Trennung von Kapitalgeber und Unternehmer erst im Zuge der Industriellen Revolution üblich wurde. Vorher wurde das Unternehmenskapital weitgehend vom Eigentümer

253 Smith (1776), S. 49.

254 Vgl. Blaug (1997), S. 96.

255 von Thünen (1875), S. 85. Vgl. auch Kirzner (1979), S. 40 ff. 
selbst oder von seiner Familie und seinen Freunden bereitgestellt. ${ }^{256}$ Die Vernachlässigung des Unternehmers leuchtet aber nicht nur bei Betrachtung der in der damaligen Gesellschaft vorherrschenden Wirtschaftsstruktur ein, sondern auch bei Berücksichtigung des inhaltlichen Hauptanliegens der klassischen Theorien. Smith und die Klassiker beschäftigen sich nahezu ausschließlich mit langfristigen Gleichgewichten. Kurzfristige Ungleichgewichte können in der klassischen Theorie nur durch außergewöhnliche Umstände entstehen. Somit existieren in der Regel langfristig keine außerordentlichen Profite, die kreative Unternehmer stimulieren können. Liegen dennoch zeitweise Ungleichgewichtszustände vor, so profitiert nicht die Mehrheit der normalen Unternehmer, sondern nur die Minderheit der Spekulanten, über die Smith schreibt: „, The speculative merchant exercises no one regular, established, or well-known branch of business. He is a corn merchant this year, and a wine merchant the next, and a sugar, tobacco, or tea merchant the year after. He enters into every trade when he foresees that it is likely to be more than commonly profitable, and he quits it when he foresees that its profits are likely to return to the level of other trades. His profits and losses, therefore, can bear no regular proportion to those of any one established and well-known branch of business. "257 Somit sind auch Unternehmensgründer für Smith Spekulanten, die zu Beginn ihrer Geschäftstätigkeit auBergewöhnliche Unternehmensgewinne erzielen können, welche sich aber mit der Zeit durch den Wettbewerb verringern. ${ }^{258}$ Im Normalfall herrscht auf allen Märkten ein Gleichgewichtszustand mit Gleichgewichtspreisen, -löhnen und -profiten auf einem angemessenen Niveau vor.

Nicht nur bei den Klassikern, sondern auch bei Marx (1904) findet sich kaum ein Hinweis auf eine besondere Unternehmerfunktion. Dabei geht Marx auf nötige Organisationsfähigkeiten des Unternehmers ein, die beispielsweise mit der Überwachung der Arbeitskräfte verbunden sind. ${ }^{259}$ Er ordnet diesen Fähigkeiten aber keine ökonomische Funktion und keinen speziellen Profitteil zu und verbindet so gleichfalls die Funktionen des Kapitalisten und des Unternehmers miteinander. ${ }^{260}$

Ebenso weist die Neoklassik dem Unternehmer keine besondere Rolle und Verantwortung zu. Nach Marshall (1920) beruhen die unternehmerischen Entscheidungen lediglich auf der individuellen Grenznutzenmaximierung. ${ }^{261}$ Es wurde damit zwar zum ersten Mal die individuelle handelnde Person in den Mit-

256 Vgl. Blaug (1997), S. 98, und Kirzner (1979), S. 43 ff., bei dem sich weitere Erklärungsansätze für die Vernachlässigung der Rolle des Unternehmers bei den englischen Klassikern finden.

257 Smith (1776), S. 116.

258 Smith (1776), S. 117.

259 Vgl. Marx (1904), S. 369 f.

260 Zur Interpretation von Marx vgl. Blaug (1997), S. 97.

261 Vgl. Marshall (1920), S. $78 \mathrm{ff}$. 
telpunkt gerückt, aber da in der Neoklassik vollständige Information und vollkommene Konkurrenz herrschen, ist der unternehmerische Entscheidungsfindungsprozess nur sehr vereinfacht dargestellt. Dem Unternehmer werden somit weder außergewöhnliche Charaktereigenschaften noch die Fähigkeit zur kreativen Ideenbildung zugebilligt. Im langfristigen Gleichgewicht, das ebenso wie bei den Klassikern den Schwerpunkt der Betrachtung bildet, gibt es überhaupt keinen Unternehmergewinn und also auch keine unternehmerische Initiative. Kapital und Arbeit erhalten nur einen Zins bzw. Lohn in Höhe ihrer Grenzprodukte. ${ }^{262}$

In der Literatur wird weitestgehend vernachlässigt, dass Keynes (1936) die Kreativität, die Vorstellungskraft und den Optimismus von Unternehmern explizit erwähnt, welche er zusammengenommen als „,animal spirits“, zu deutsch Lebensgeister, bezeichnet. ${ }^{263}$ „Even apart from the instability due to speculation, there is the instability due to the characteristic of human nature that a large proportion of our positive activities depend on spontaneous optimism rather than on a mathematical expectation, whether moral or hedonistic or economic. Most, probably, of our decisions to do something positive, the full consequences of which will be drawn out over many days to come, can only be taken as a result of animal spirits - of a spontaneous urge to action rather than inaction, and not as the outcome of a weighted average of quantitative benefits multiplied by quantitative probabilities. " 264 Ein Unternehmer braucht demnach diese „animal spirits“ für eine erfolgreiche Geschäftstätigkeit. Die reine Berechnung der Produktions- und Gewinnmöglichkeiten reicht nicht aus. Abgesehen von diesen kurzen Ausführungen vernachlässigt Keynes jedoch die Figur des Unternehmers. Der Keynesianismus wie auch sein Gegenstück der Monetarismus beschränken sich auf eine fast ausschließlich makroökonomische Perspektive der Volkswirtschaft, in welcher die Unternehmer - ebenso wie die anderen Akteure - nur passiv auf die Marktsignale reagieren.

Zahlreiche empirische Untersuchungen weisen jedoch darauf hin, dass die Funktion des Unternehmers bzw. die unternehmerische Aktivität in der Gesellschaft in engem Zusammenhang mit der Ausprägung des Wirtschaftswachstums steht. ${ }^{265}$ Die räumliche Verteilung von Unternehmertalent bestimmt in hohem Maße die Wachstumsraten. ${ }^{266}$ Der Unternehmer scheint also durchaus auch eine aktive Rolle einzunehmen. Dennoch besitzt er weder in der neoklassischen noch in der keynesianischen Theorie eine herausragende Rolle, weil im Modell des

262 Vgl. Marshall (1920), S. 426 ff. Zur Interpretation vgl. Berghoff (2004), S. 32 f.; Blaug (1997), S. $99 \mathrm{ff}$.

263 Eine dieser Ausnahmen bildet Gifford (2005), S. 41.

264 Keynes (1936), S. 161.

265 Vgl. Baumol (1968), S. 65 f.; Audretsch und Fritsch (2003). Für Entwicklungsländer finden sich widersprüchliche empirische Ergebnisse, vgl. van Stel et al. (2005), S. $318 \mathrm{f}$.

266 Vgl. Bianchi und Henrekson (2005), S. 360. 
gewinnmaximierenden Unternehmens kein Platz für unternehmerische Initiative vorgesehen ist. Es gibt Entscheidungsvariablen wie Preise und Outputmengen, die optimiert werden, aber nur im Rahmen der grundlegenden Mechanismen und vorgegebenen Beziehungen zwischen den Variablen. Unternehmerische Initiative lässt sich in eine solche Modellierung nicht integrieren. ${ }^{267} \mathrm{Da}$ der Keynesianismus in makroökonomischer Hinsicht und die Neoklassik im Bereich der Mikroökonomie auch heute noch die Standardparadigmen der Volkswirtschaftslehre darstellen, wird die Figur des Unternehmers bis heute in weiten Teilen der Wissenschaft vernachlässigt - mit Ausnahme der so genannten EntrepreneurshipTheorie.

\subsection{Grundlegendes zum Unternehmer und zum Entrepreneur}

Bevor mit Hilfe der Entrepreneurship-Theorie die Fragestellungen behandelt werden, wie sich zum einen Unternehmer in ihren Eigenschaften und Fähigkeiten von anderen Wirtschaftssubjekten unterscheiden und wie sich zum anderen gewöhnliche Unternehmer von besonderen „Entrepreneuren“ unterscheiden, muss geklärt werden, welche Wirtschaftssubjekte als Unternehmer und damit potentielle „Entrepreneure“ überhaupt in den Anwendungsbereich der Entrepreneurship-Theorie fallen. Da die Entrepreneurship-Theorie eine relativ junge Theorierichtung ist, die aus volks- und betriebswirtschaftlichen, psychologischen und soziologischen Elementen besteht, gibt es noch keine allgemein verbindlichen Begrifflichkeiten. Selbst bei den zentralen Begriffen herrscht noch Uneinigkeit. ${ }^{268}$ Deswegen muss eine grundlegende Untersuchung der Abgrenzungsmöglichkeiten des Unternehmerbegriffs im Rahmen der EntrepreneurshipTheorie erfolgen, die mit den Anfängen dieser Theorierichtung beginnt. Um die Begrifflichkeiten in dieser Arbeit klar voneinander abzugrenzen, wird hier zwischen dem gewöhnlichen Unternehmer und dem später genauer zu definierenden Entrepreneur $^{269}$ unterschieden, der sich durch bestimmte Fähigkeiten und Eigenschaften auszeichnet (vgl. Abschnitte 5.4, 5.5 und 5.6).

\subsubsection{Der Begriff des Unternehmers}

Als ursprüngliche Bezeichnung des Unternehmers stammt der Begriff des Entrepreneurs aus dem Französischen und wurde zuerst für Anführer von Militär-

267 Vgl. Baumol (1968), S. 66 ff.; Blaug (1997), S. 102 ff.

268 Vgl. McMullen et al. (2007), S. 9 ff.; Drucker (1985), S. 21 ff.; Henrekson (2007), S. 719.

269 Dabei bleibt es leider nicht aus, dass bei der Verwendung von Originalzitaten aus dem Englischen bzw. Französischen vom „entrepreneur“ gesprochen wird, jedoch nur der Unternehmer im Sinne der hier vorgenommenen Begriffsverwendung gemeint ist. 
expeditionen sowie Architekten und Unternehmer von staatlichen Bauprojekten verwendet. ${ }^{270}$ Einer der ersten Ökonomen, der diesen Begriff benutzte, ist Say (1803), der als erster die Fähigkeit des Unternehmers als Entscheidungsträger und Organisator betonte. ${ }^{271}$ Der Unternehmer koordiniert den Einsatz der Produktionsfaktoren und ist für die Produktion seiner Güter verantwortlich. Er ist Nachfrager auf den Faktormärkten und Anbieter auf den Gütermärkten und ist für die gesamte Organisation des Unternehmens verantwortlich: „Ceux qui disposent de l'une de ces trois sources de la production [Mit den drei Produktionsfaktoren sind Kapital, Boden und Industrie gemeint, wobei Say unter dem Begriff ,industrie“ jegliche Form von Arbeit, also auch die Arbeit des Unternehmers, versteht.] sont marchands de cette denrée que nous appelons ici services productifs; les consommateurs des produits en sont les acheteurs. Les entrepreneurs d'industrie ne sont, pour ainsi dire, que des intermédiaires qui réclament les services productifs nécessaires pour tel produit en proportion de la demande qu'on fait de ce produit. ${ }^{\prime 272}$

Der Profit des Unternehmers besteht aus zwei Teilen. Einen Teil erhält der Unternehmer für seine Arbeitskraft (,,industrie "), den anderen Teil erhält er für sein eingesetztes Kapital. ${ }^{273}$ Im Gegensatz zu Smith grenzt Say also die beiden Formen des Profits gegeneinander ab und trennt klar zwischen der Funktion des Kapitalisten und des Organisators. Die Arbeitskraft des Unternehmers beschreibt Say als „,ses facultés industrielles, c'est-à-dire à son jugement, à ses talents naturels ou acquis, à son activité, à son esprit d'ordre et de conduite “274 bzw. „du jugement, de la constance, la connaissance des hommes et des choses. Il s'agit d'apprécier convenablement l'importance de tel produit, le besoin qu'on en aura, les moyens de production; il s'agit de mettre en jeu quelquefois un grand nombre d'individus; il faut acheter ou faire acheter des matières premières, réunir des ouvriers, chercher des consommateurs, avoir un esprit d'ordre et d'économie, en un mot, le talent d'administrer. " ${ }^{\prime 275}$ Die bestimmende Eigenschaft des Unternehmers ist also seine Organisationsfähigkeit. Der Unternehmer koordiniert die Produktion, indem er Rohstoffe und Vorprodukte einkauft, die die Arbeitskräfte verarbeiten. Es obliegt ihm außerdem, seine Produkte an den Markt zu bringen, wo sie dem Geschmack der Nachfrager entsprechen müssen, um verkauft werden zu können. Dabei geht Say zwar auch auf das mit der Unternehmertätigkeit zwingend zusammenhängende Risiko (vgl. Abschnitt 5.5) eines Vermögens- oder Ansehensverlustes kurz ein, ${ }^{276}$ die organisatorischen

270 Vgl. Berghoff (2004), S. 33; Blaug (1997), S. 96; Kirzner (1979), S. 39.

271 Vgl. Berghoff (2004), S. 33 f.; Schumpeter (1926), S. 113.

272 Say (1861), S. 343.

273 Vgl. Say (1861), S. 346.

274 Say (1861), S. 362 f.

275 Say (1861), S. 364.

276 Vgl. Say (1861), S. 364. 
Fähigkeiten des Unternehmers stehen jedoch eindeutig im Mittelpunkt seiner Analyse. Somit liegt die Interpretation nahe, dass Say nicht die Funktion des Unternehmenseigentümers, sondern die des obersten Entscheidungsträgers (im Sinne des Geschäftsführers) als maßgeblich für den Unternehmer ansieht. Unternehmer ist damit derjenige, der die Geschäftstätigkeit des Unternehmens koordiniert und organisiert.

Die Organisationsfähigkeit des Unternehmers steht in Anknüpfung an Say auch im Mittelpunkt der Arbeit von Baumol (1968). Allerdings nimmt Say eine Abgrenzung von Kapitalist und Organisator vor, während Baumol zwischen den Funktionen des Managers und denen des Unternehmers trennt. Der Manager kümmert sich um die Effizienz der verwendeten Arbeitsprozesse und sorgt dafür, dass das Unternehmen sein Produktionspotential ausschöpft. Der Unternehmer hat dagegen eine andere Funktion: Er kommt auf neue Ideen und realisiert diese. Seine Aufgabe lässt sich eher als Führung des Unternehmens im Sinne des Gebens von Inspirationen beschreiben. ${ }^{277}$ Auch bei Baumol zielt der Begriff des Unternehmers also nicht zwingend auf den Eigentümer, sondern wiederum eher auf den obersten Entscheidungsträger im Unternehmen.

Auch Casson (1992) geht auf das Abgrenzungsproblem zwischen Eigentümern und Managern ein und kommt in seiner Definition des Unternehmers zu einem ähnlichen Schluss wie Say und Baumol. Für Casson sind Entscheidungskompetenz und Entscheidungsgewalt ausschlaggebend dafür, welche Wirtschaftssubjekte als Unternehmer bezeichnet werden können. So können sowohl Angestellte in leitenden Positionen als auch Eigentümer eines Unternehmens abhängig von ihrer Kompetenz, Entscheidungen zu treffen und der Gewalt, diese auch durchzusetzen - als Unternehmer bezeichnet werden. ${ }^{278}$

In dieser Arbeit bezieht sich der Begriff des Unternehmers in Anlehnung an die genannten Autoren auf denjenigen, der die wesentlichen strategischen Entscheidungen des Unternehmens persönlich trifft. Bei Personengesellschaften ist der Unternehmer typischerweise der Eigentümer-Unternehmer oder ein Gesellschafter, bei Kapitalgesellschaften der Geschäftsführer oder der Vorstand. Bei beiden Rechtsformen können aber auch Angestellte in leitender Position als Unternehmer wirken.

\subsubsection{Die Unterscheidung zwischen Unternehmer und Entrepreneur}

Die Hauptfragestellung der Entrepreneurship-Theorie besteht darin, was einen Unternehmer im eben beschriebenen Sinne hinsichtlich seiner Eigenschaften und Fähigkeiten von anderen Wirtschaftssubjekten unterscheidet, die nicht unternehmerisch tätig werden. Der grundlegende Gedanke, der hinter den vorliegenden Ausführungen steht, besteht in Anlehnung an Sarasvathy (2004) jedoch

277 Vgl. Baumol (1968), S. $64 \mathrm{f}$.

278 Vgl. Casson (1992), S. $190 \mathrm{f}$. 
darin, dass auch die Gruppe der Unternehmer nicht homogen ist: „If we focus our work on what is common across all entrepreneurs as opposed to nonentrepreneurs, we would be falling into the mire of typology thinking. Instead, if we were to turn our attention to key differences within any given type of entrepreneurs, we will begin to characterize entrepreneurship in all its variety and yet find ways to deal with it in a useful manner in our research and pedagogy. “279

So entsprechen manche Unternehmer stärker als andere der - in dieser Arbeit als Entrepreneur bezeichneten - Idealvorstellung des Unternehmers, welche im Rahmen verschiedener Theorieansätze jeweils unterschiedlich beschrieben wird. Gemeinsam ist diesen Ansätzen aber, dass sie Kriterien für die Untersuchung liefern, durch welche Eigenschaften und Fähigkeiten sich Unternehmer und Entrepreneure unterscheiden. Bevor die Anwendung dieser Entrepreneurship-Theorieansätze auf die Thematik Innovationsförderung erfolgt, wird im nächsten Abschnitt das Konzept der „entrepreneurial opportunities“ erläutert. Dieses Konzept integriert alle für diese Arbeit wesentlichen EntrepreneurshipTheorieansätze und bildet damit den Rahmen der folgenden Untersuchung.

\subsection{Unternehmerische Gelegenheiten (,entrepreneurial opportu- nities") und Innovationsförderung}

Obwohl wie erwähnt selbst bei den zentralen Begriffen der EntrepreneurshipTheorie noch Uneinigkeit herrscht, so stellen die ,entrepreneurial opportunities " (im Folgenden als EO abgekürzt) von Shane und Venkataraman (2000) das wohl einzige in der Entrepreneurship-Literatur allgemein anerkannte Paradigma dar. ${ }^{280}$ Die Entrepreneurship-Theorie definieren die Autoren als ,, the scholarly examination of how, by whom, and with what effects opportunities to create future goods and services are discovered, evaluated, and exploited ". ${ }^{281}$ EO stellen profitversprechende Möglichkeiten für den Unternehmer dar, genauer ,those situations in which new goods, services, raw materials, and organizing methods can be introduced and sold at greater than their cost of production" 282 . Aufgrund von derzeit nicht in den Preisen enthaltenen Informationen hat der Unternehmer eine Vorstellung von einer möglicherweise verbesserten Ressourcenallokation und erschafft neue Ziel-Mittel-Beziehungen, die die ökonomischen Tauschprozesse verändern. ${ }^{283}$ Casson und Wadeson (2007) bauen auf den Ergebnissen von Shane und Venkataraman auf und definieren eine EO als , a po-

279 Sarasvathy (2004), S. 711.

280 Vgl. Grichnik (2006), S. 1304; Acs und Audretsch (2005), S. 5.

281 Shane und Venkataraman (2000), S. 218.

282 Shane und Venkataraman (2000), S. 220.

283 Vgl. Eckhardt und Shane (2003), S. $336 \mathrm{ff}$. 
tentially profitable but hitherto unexploited project “284. Dass bislang nicht ausgebeutete EO in einer effizienten Volkswirtschaft überhaupt existieren, deutet auf die Existenz von Entdeckungskosten hin. Unternehmer wenden nur dann Ressourcen für die Suche nach EO auf, wenn sie einen ausreichenden Profit erhoffen, der die Entdeckungskosten übertrifft. ${ }^{285}$

Die EO stellen den zentralen Anknüpfungspunkt der EntrepreneurshipTheorie dar, da ihre Ausbeutung ein entscheidendes Kriterium für Unternehmertum ist. So beziehen sich EO zum einen auf die Gründung neuer Organisationen, z. B. eines Unternehmens, und zum anderen auf Möglichkeiten innerhalb von existierenden Organisationen. ${ }^{286}$ Es gibt einen bedeutenden Literaturzweig, der sich auf die erste Alternative konzentriert und somit die EntrepreneurshipTheorie ausschließlich auf Unternehmensgründungen als EO bezieht. Unter den Schlagwörtern „,business start-ups “ bzw. „nascent entrepreneurs" werden die Eigenschaften und Fähigkeiten von zukünftigen und tatsächlichen Unternehmensgründern analysiert. ${ }^{287}$ Von diesem Verständnis geht beispielsweise der Global Entrepreneurship Monitor aus, ein Forschungskonsortium, das sich mit Unternehmertum in zur Zeit 42 Ländern auf der Welt beschäftigt. ${ }^{288}$ Ein anderer beachtlicher Literaturzweig betrachtet die zweite Alternative insbesondere mit Blick auf die Ausnutzung von EO durch Unternehmenseigentümer. $\mathrm{Zu}$ diesem Literaturbereich zählen empirische Untersuchungen aus dem EntrepreneurshipBereich, die die Selbständigenquote eines Landes oder einer Region als Indikator für Unternehmertum verwenden. ${ }^{289}$

Eine solche isolierte Betrachtung wird dem offenen Konzept der EO jedoch nicht gerecht. Unter den Begriff der EO als Anknüpfungspunkt der Entrepreneurship-Theorie fallen auch Innovationen, da sie potentiell profitable Möglichkeiten innerhalb existierender Unternehmen oder auch im Rahmen von Unternehmensgründungen darstellen. ${ }^{290}$ Die Voraussetzung für eine Innovation ist eine bestimmte Invention, die die Chance einer Durchsetzung auf dem Markt birgt. Der Unternehmer muss nun diese EO entdecken und zunächst auf die Invention stoßen und diese als Chance erkennen. Daran anschließend muss sich der Unternehmer entscheiden, ob er die Invention ausbeuten will. Falls er sich

284 Casson und Wadeson (2007), S. 286.

285 Vgl. Casson und Wadeson (2007), S. 286.

286 Vgl. Shane und Venkataraman (2000), S. 219; Acs und Audretsch (2005), S. 6; Grichnik (2006), S. 1305 ff.; McMullen et al. (2007), S. 17 ff.; Buenstorf (2007), S. $326 \mathrm{f}$.

287 Vgl. u. a. Heyden (2002), S. 20; Lückgen et al. (2006), S. 9; Santarelli und Vivarelli (2007); Fallgatter (2004), S. 24 f.

288 Vgl. Bosma et al. (2008), S. 9, sowie die Internetseite des Global Entrepreneurship Monitor, in: http://www.gemconsortium.org, zugegriffen am 28.04.2008.

289 Vgl. z. B. Blanchflower und Oswald (1998).

290 Vgl. Eckhardt und Shane (2003), S. 340; Casson und Wadeson (2007), S. 294; Companys und McCullen (2007), S. 308. 
für die Ausbeutung der EO entscheidet, muss er sich zwischen verschiedenen möglichen Arten der Ausbeutung entscheiden. Wenn der Unternehmer beispielsweise nicht über ausreichende Eigenmittel verfügt, so muss er Finanzierungsquellen finden, um die Innovationstätigkeit aufnehmen zu können, zu denen auch Innovationsfördermittel zählen können. ${ }^{291}$

Innovationsförderung selbst kann aber ebenfalls als EO bezeichnet werden. Innovationsfördermittel sind eine potentielle Finanzierungsquelle für alle innovativen Unternehmen, die die speziellen Kriterien des Förderprogramms erfüllen. Allerdings bewerben sich nicht alle in Frage kommenden Unternehmer für das Programm. Es bewerben sich nur diejenigen Unternehmer, die das Förderprogramm entdeckt haben - entweder weil sie aktiv nach einem Programm gesucht haben oder weil sie von dritter Seite auf das Programm hingewiesen wurden. An die Entdeckung des Programms schließt sich dann die bewusste Entscheidung für die Bewerbung und die für eine Bewerbung aufzuwendenden Ressourcen an. Die erfolgreiche Ausbeutung der EO besteht in einer tatsächlichen Förderung des Unternehmens durch das Programm, wobei diese nicht nur von der Qualität der Bewerbung sondern auch von der Bewertung durch die zuständigen Institutionen abhängt.

In den folgenden Abschnitten 5.4, 5.5 und 5.6 werden anhand der Tätigkeitsfelder Innovation und dem Erhalt von Innovationsförderung die drei verschiedenen Phasen der EO (Entdeckung, Bewertung und Ausbeutung) näher ausgeführt. Theorieansätze aus dem Entrepreneurship-Bereich werden entsprechend dieser drei Phasen klassifiziert und vorgestellt. Dabei wird zunächst untersucht, inwiefern sich Unternehmer, die die EO Innovation ergreifen, von nicht innovativ tätigen Unternehmern unterscheiden. Des Weiteren wird beleuchtet, inwiefern sich Unternehmer, die die EO Innovationsförderung ergreifen, von nicht geförderten Unternehmern unterscheiden. Dabei ist insbesondere die Fragestellung interessant, ob die Entrepreneurship-Theorie Anhaltspunkte für die Erklärung bieten kann, welche Unternehmer sich für Innovationsförderung bewerben und inwiefern Unternehmer, die die EO Innovationsförderung ergreifen, als idealtypische Unternehmer - Entrepreneure - bezeichnet werden können.

$\mathrm{Da}$ in der folgenden Untersuchung die EO Innovationsförderung im Fokus steht, werden diejenigen Entrepreneurship-Theorieansätze, welche sich nicht in den Rahmen der EO einordnen lassen, im Folgenden vernachlässigt. Sie würden für die weitere Analyse keinen zusätzlichen Erkenntnisgewinn liefern. Dazu zählt beispielsweise das Modell des ,Jack of all trades “ von Lazear (2005), das den Unternehmer als Hansdampf in allen Gassen beschreibt, der jede gute Gelegenheit nutzt. In Lazears Modell besitzt der Unternehmer im Gegensatz zum spezialisierten Angestellten eine ausgewogene Mischung von verschiedenen Fä- 
higkeiten. ${ }^{292}$ Des Weiteren werden die Untersuchungen, die den Einfluss von soziodemographischen Variablen (z. B. Geschlecht, Alter, Schulbildung und Beruf der Eltern) und regionalen Variablen (z. B. Bevölkerungsdichte und Landpreise) auf die Ausprägung des Unternehmertums untersuchen, im Folgenden nicht ausgeführt. ${ }^{293}$ Die psychologischen Untersuchungen der Traits School beschäftigen sich mit persönlichen Eigenschaften der Unternehmer wie beispielsweise der Kontrollüberzeugung (,locus of control ") und dem Bedürfnis nach Leistung (,,need for achievement") und werden ebenfalls im Folgenden vernachlässigt. ${ }^{294}$ Diesen Theorieansätzen ist im Übrigen gemein, dass sie sich vorwiegend mit der speziellen EO Unternehmensgründung beschäftigen und nicht mit EO im Allgemeinen. Deswegen konzentrieren sich die folgenden Abschnitte auf diejenigen Entrepreneurship-Theorieansätze, die auf jegliche Arten von $\mathrm{EO}$ anzuwenden sind.

\subsection{Erste Phase: Entdeckung der EO}

Zunächst muss der Unternehmer eine EO entdecken, bevor er sie in einer späteren Phase ausschöpfen kann. Die Tatsache, dass bestimmte Unternehmer eine EO entdecken und andere nicht, hängt erstens mit dem Besitz unterschiedlicher Informationen und zweitens mit den kognitiven Informationsverarbeitungsfähigkeiten der Wirtschaftssubjekte zusammen. ${ }^{295}$ Aufgrund der individuell in der Vergangenheit gemachten Erfahrungen und der daraus gesammelten Informationen stößt ein Unternehmer auf eine ganz bestimmte EO, während ein anderer Unternehmer diese EO nicht entdeckt. Dabei ist die Entdeckung der EO meist nicht das Resultat einer gezielten Suche, sondern erfolgt zufällig durch eine Kombination von Informationen aus den verschiedensten Bereichen. Wäre die EO offensichtlich, so wäre sie bereits von anderen Unternehmern ergriffen worden. ${ }^{296}$ Der Prozess zur Entdeckung einer EO besteht aus zwei Phasen. Erstens identifiziert der Unternehmer die Gebiete, auf die er sich in seiner Suche konzentriert. Zweitens analysiert der Unternehmer innerhalb dieser Gebiete detailliert einige EO und ihre Charakteristika. Dabei bezieht er sowohl seine eigenen Präferenzen und Fähigkeiten in die Analyse mit ein als auch die ökonomische Umgebung und den Erfolg bereits durchgeführter EO. ${ }^{297}$

292 Vgl. Lazear (2005).

293 Vgl. z. B. Lückgen et al. (2006); Evans und Leighton (1989).

294 Vgl. z. B. Evans und Leighton (1989); Cools und van den Broeck (2006); Stewart Jr. und Roth (2007); Licht (2007), S. 827 ff.

295 Vgl. Shane und Venkataraman (2000), S. 221 f.; Companys und McCullen (2007), S. 304 ff.; Buenstorf (2007), S. 326.

296 Vgl. Shane (2000), S. $451 \mathrm{ff}$.

297 Vgl. Casson und Wadeson (2007), S. $293 \mathrm{ff}$. 
Der erste Bereich der im Folgenden vorgestellten EntrepreneurshipTheorieansätze beschäftigt sich daher mit den Fähigkeiten zur Informationsverarbeitung und der Meinungsbildung von Unternehmern. Schon Say wies darauf hin, wie wichtig es für den Unternehmer ist, Informationen über die verschiedenen Aspekte seines Geschäfts zu besitzen: „Non-seulement il faut connaître la nature et les qualités des marchandises sur lesquelles on spécule, mais encore se former une idée de l'étendue des besoins et des débouchés aux lieux où l'on se propose de les vendre. Il faut en conséquence se tenir constamment au courant des prix de chacune de ces marchandises en différents lieux du monde. [...] Il faut connaitre les moyens de transport, la mesure des risques qu'ils entrấnent, le montant des frais qu'ils occasionnent; les usages, les lois qui gouvernent les peuples avec qui l'on a des relations; enfin il faut avoir assez de connaissance des hommes pour ne point se tromper dans les confiances qu'on leur accorde, dans les missions dont on les charge, dans les rapports quelconques qu'on entretient avec eux. “298 Der Unternehmer benutzt also nicht nur vielfältige Informationen über Produktqualität, Transportmittel, Produktpreise, sondern besitzt auch eine gute Menschenkenntnis im Rahmen seiner Geschäftsbeziehungen. Es ist wichtig, dass er sich permanent auf dem Laufenden hält (,,se tenir constamment au courant"), was die laufende Entwicklung der genannten Faktoren angeht.

In Says Analyse des Unternehmertums nimmt der Aspekt der Informationsverarbeitung insgesamt jedoch eine eher untergeordnete Rolle ein, wichtiger ist ihm die Organisationsfähigkeit des Unternehmers, wie in Abschnitt 5.2.1 erläutert wurde. Dagegen betonen die Vertreter der Österreichischen Schule den Informationsbeschaffungs- und -verarbeitungsaspekt ungleich stärker. Ihre Definition eines idealtypischen Unternehmers basiert darauf, dass dieser auf neue ökonomische Möglichkeiten stößt und in einer besonderen Art und Weise auf diese reagiert. Das unterscheidet den Entrepreneur vom gewöhnlichen Unternehmer. Zu den wichtigsten Vertretern der Österreichischen Schule zählen von Hayek, von Mises und Kirzner, deren Arbeiten im Folgenden kurz vorgestellt werden. Casson ist als wesentlicher moderner Vertreter dieses Theorieansatzes zu nennen.

\subsubsection{Die Reaktion auf die komplexe Umgebung nach von Hayek}

Von Hayek (1945) wies als erster auf das Problem der unvollständigen Information hin. Wenn ein Wirtschaftssubjekt eine Entscheidung trifft, ist es nicht in der Lage alle bekannten Informationen aufzunehmen und in seine Entscheidungsfindung mit einzubeziehen. Dazu ist die Umgebung zu komplex und es existieren zu viele Informationen. Das Individuum versucht sich auf die für seine Entscheidung relevanten Informationen zu konzentrieren, dabei kann es nur einen 
Bruchteil der tatsächlich verfügbaren Informationen nutzen und diese interpretieren. ${ }^{299}$

Als Beispiele für Unternehmer, die Informationsvorteile besitzen und diese zu ihrem Vorteil nutzen, gibt von Hayek folgende Berufe an: „And the shipper who earns his living from using otherwise empty or half-filled journeys of tramp-steamers, or the estate agent whose whole knowledge is almost exclusively one of temporary opportunities, or the arbitrageur who gains from local differences of commodity prices, are all performing eminently useful functions based on special knowledge of circumstances of the fleeting moment not known to others. ${ }^{\text {" }} 000$ Von Hayek betont, dass es auch für die Gesellschaft insgesamt wichtig und vorteilhaft ist, dass diese Individuen ihre Informationsvorteile nutzen. ${ }^{301}$ Simon greift von Hayeks Gedanken zur komplexen Umgebung des Unternehmers auf und erweitert ihn auf alle Wirtschaftssubjekte zu seiner Theorie der „bounded rationality“ (vgl. Abschnitt 3.1.1).

\subsubsection{Die Aufmerksamkeit des Unternehmers nach von Mises und Kirzner}

Der Unternehmer, der mit einer bestimmten Absicht verschiedene Produktionsfaktoren zu einem Ganzen zusammenführt, ist laut von Mises (1949) die Triebkraft des ökonomischen Marktes. ${ }^{302}$ Von Mises definiert Unternehmer als ,, acting men exclusively seen from the aspect of the uncertainty inherent in every action " 303 bzw. ,Entrepreneur means acting man in regard to the changes occurring in the data of the market "“304. Von Mises erwähnt nicht nur den Aspekt der in allen Situationen innewohnenden Unsicherheit. Gleichzeitig betont er die Notwendigkeit des Aktivwerdens des Unternehmers unter Einbeziehung der veränderten Rahmenbedingungen und Marktdaten: „Like every acting man, the entrepreneur is always a speculator. He deals with the uncertain conditions of the future. His success or failure depends on the correctness of his anticipation of uncertain events. " ${ }^{305}$ Der Unternehmererfolg hängt also von der Entwicklung der mit Unsicherheit behafteten Ereignisse und Rahmenbedingungen ab. Denn wenn alle Unternehmer die unsichere Zukunft korrekt vorhersagen könnten, gäbe es weder Gewinne noch Verluste. Unternehmergewinn und Unsicherheit hängen zwingend zusammen, entscheidend sind aber die Bewertung der unsi-

299 Vgl. von Hayek (1945), S. 521 ff.

300 von Hayek (1945), S. 522.

301 Vgl. von Hayek (1945), S. 522.

302 Vgl. von Mises (1949), S. 249.

303 von Mises (1949), S. 254.

304 von Mises (1949), S. 255.

305 von Mises (1949), S. 288. 
cheren Rahmenbedingungen und die daraus resultierenden Aktionen der Unternehmer. ${ }^{306}$

Eine eng verwandte Sicht des Unternehmers findet sich bei Kirzner (1973). Er definiert Unternehmertum als Element der menschlichen Entscheidungsfindung über die Wahrnehmung von neuen Rahmenbedingungen bzw. "that element of alertness to possibly newly worthwhile goals and to possibly newly available resources “ ${ }^{307}$. Dieses unternehmerische Element sei elementar, um die menschliche Entscheidungsfindung zu verstehen, die nicht als reine Kalkulation bzw. als mechanische Nutzenmaximierung aufgefasst werden kann, weil die Welt zu komplex für eine rein mechanische Optimierung ist. ${ }^{308}$

Die Aufmerksamkeit bzw. Wachsamkeit (,alertness") des idealtypischen Kirzner'schen Entrepreneurs ist allerdings nicht zu verwechseln mit seinen Kenntnissen bzw. seinem Wissen (,knowledge“). Denn der Begriff Aufmerksamkeit sagt aus, dass der Entrepreneur weiß, wo er seine Informationen erhält es geht nicht darum, dass er selbst Informationen anhäuft. So kann ein Entrepreneur auch Angestellte anwerben und beschäftigen, die sehr gut informiert sind oder die selber diese ,alertness" besitzen und sie für das Unternehmen und dessen Vorteil einsetzen. Mit ihrer Beschäftigung beweist der Entrepreneur einen höheren Grad an „alertness“ als seine Angestellten. ${ }^{309}$ Kirzner weist hiermit auf einen Punkt hin, der für die hier vorgenommene Untersuchung zentral ist: Der idealtypische Unternehmer muss selbst kein Wissen anhäufen, sondern er muss die richtigen Informationsquellen kennen, aus denen er sein Wissen bei Bedarf erhält. Aus dieser Definition des Entrepreneurs über seine „alertness“ wird deutlich, dass die Frage nicht pauschal beantwortet werden kann, welche Personengruppe im Unternehmen Entrepreneur im Kirzner'schen Sinne sein kann. Entrepreneur nach Kirzner ist nämlich einzig und allein derjenige, der die verdeckten Gewinnmöglichkeiten erkennt und ausnutzt. Das kann der Unternehmenseigentümer oder der Geschäftsführer/Manager des Unternehmens sein, der im Vergleich zu seinen Konkurrenten Gewinnmöglichkeiten erkennt. Das kann aber auch ein untergeordneter Angestellter sein, der unbeobachtet vom Manager Gewinnmöglichkeiten auftut, die anderen verborgen bleiben. Wenn der Angestellte die Gewinnmöglichkeiten nicht für das Unternehmen ausnutzt, sondern am Manager und somit am Unternehmen vorbei für sich selbst realisiert, ist er ein Entrepreneur. $^{310}$

Kirzner verwirft die Theorie des reinen Homo Oeconomicus, ohne diese beim Namen zu nennen. Stattdessen entwirft er in groben Zügen ein neues Unternehmerbild, nämlich das des Entscheidungsträgers, dessen Rolle im Wirt-

306 Vgl. von Mises (1949), S. $291 \mathrm{ff}$.

307 Kirzner (1974), S. 35.

308 Vgl. Kirzner (1974), S. 32 ff.

309 Vgl. Kirzner (1974), S. 67 ff.; Kirzner (1979), S. 8.

310 Vgl. Kirzner (1974), S. 55 ff. 
schaftsprozess vollständig durch dieses unternehmerische Element beschrieben werden kann. ${ }^{311}$ Der Unternehmer muss Informationen über Preisdifferenzen auf Faktor- und Produktmärkten sammeln und auswerten und auf der Basis dieser Informationen schnelle Entschlüsse fassen. Nur darin besteht die unternehmerische Tätigkeit. Der Unternehmer muss selbst keine Ressourcen in den Produktionsprozess einbringen und unterscheidet sich so vom Produzenten, welcher die Produktion koordiniert und seine Organisationsfähigkeit als Inputfaktor in die Produktion einfügt. ${ }^{312}$ Der reine unternehmerische Profit besteht aus der Differenz zwischen zwei Preisen: der Bereitschaft des Verkäufers zu einem niedrigeren Preis zu verkaufen und der Bereitschaft des Käufers zu einem höheren Preis zu kaufen. Der Unternehmer muss nur diese Verkäufer und Käufer und die für ihn aus den Preisunterschieden resultierenden Gewinnmöglichkeiten finden: "The discovery of a profit opportunity means the discovery of something attainable for nothing at all.

Nur durch unvollkommene Information und die daraus resultierenden Marktungleichgewichte gibt es diese Profitmöglichkeiten für den Unternehmer. ${ }^{314}$ In Kirzners Modell herrscht keine vollkommene Information, so dass ebenso wie bei von Mises Entscheidungen unter Unsicherheit getroffen werden. Die Übernahme der Unsicherheit ist dabei nicht das entscheidende Element. Stattdessen ist die spezifische Eigenschaft, die in Kirzners Modell den Entrepreneur von anderen Wirtschaftssubjekten unterscheidet, seine Fähigkeit auf neue Möglichkeiten zu stoßen: „Entrepreneurship does not consist of grasping a free ten-dollar bill which one has already discovered to be resting in one's hand; it consists in realizing that it is in one's hand and that it is available for the grasping. “315

Kirzners Kritiker sind allerdings der Ansicht, dass die Unternehmertätigkeit nicht nur aus reiner Arbitrage bzw. Spekulation aus der Ausnutzung von Preisdifferenzen besteht, sondern dass der Unternehmer durch seine Tätigkeit selbst produktiv zum Entstehen des Unternehmergewinns beiträgt. ${ }^{316}$ Die im Folgenden ausgeführte Definition des Entrepreneurs von Casson vollzieht diesen weitergehenden Schritt, indem die Urteilsfähigkeit des Unternehmers und sein Informationsstand in die Analyse des Unternehmergewinns mit einbezogen werden. In diesem Zusammenhang kann wiederum eine Differenzierung innerhalb der Gruppe der Unternehmer vorgenommen werden. Dagegen weisen die dargestellten Ausführungen von Kirzner hauptsächlich auf die Unterscheidung von Unternehmern und anderen Wirtschaftssubjekten hin.

311 Vgl. Kirzner (1974), S. 41.

312 Vgl. Kirzner (1974), S. 44 f.

313 Kirzner (1974), S. 48.

314 Vgl. Kirzner (1979), S. 4 ff.

315 Kirzner (1974), S. 47.

316 Vgl. Blaug (1997), S. 106. 


\subsubsection{Moderne Theorieansätze: die Urteilsfähigkeit nach Casson}

Casson $(1992,2001,2003)$ definiert den idealtypischen Unternehmer über seine „Fähigkeit zur Urteilsbildung in wirtschaftlichen Entscheidungsprozessen "317, d. h. ,, an entrepreneur is someone who specializes in taking judgmental decisions about the coordination of scarce resources "318. Um eine Entscheidung bezüglich der eingesetzten Produktionsfaktoren, der zu tätigenden Investitionen und der zu produzierenden Güter zu treffen, muss der Unternehmer improvisieren und kann sich nicht nur auf Routinen verlassen. Casson'sche Entrepreneure investieren in Produkte und Projekte, in die andere nicht investieren. Sie besitzen private Informationen über die fraglichen Projekte, die das Investitionsrisiko geringer erscheinen lassen, auf die aber gleichzeitig nicht uneingeschränkt Verlass ist.

Cassons Ansatz erfasst neben der Urteilsfähigkeit des Unternehmers in besonderem Maße die Bedeutung der Informationsströme: „, In diesem Kontext lassen sich Unternehmer als Spezialisten betrachten, die über die Fähigkeit verfügen, Informationen mit der Aussicht auf Gewinn zu synthetisieren, indem sie Daten, Konzepte und Ideen auswerten, deren Bedeutung anderen Menschen nicht immer bewusst ist. "319 Der Unternehmer benötigt nur wenig Zeit für das Treffen einer Entscheidung, doch muss er viel Zeit in die Informationssuche und -verarbeitung investieren. ${ }^{320} \mathrm{Da}$ die wirtschaftliche Umgebung volatil ist, muss sich der Unternehmer immer neue Informationen besorgen, um bei seinen Investitionsentscheidungen auf langfristige Entwicklungen und Veränderungen reagieren und gewinnbringende Möglichkeiten nutzen zu können. Er kann seine Entscheidungskompetenz an Untergebene delegieren, wenn es sich um kurzfristige Schocks und Entwicklungen handelt, auf die die Mitarbeiter mit Routinen reagieren können. Die besondere Entscheidungsfähigkeit eines Entrepreneurs kommt in denjenigen Situationen zum Tragen, in denen keine Routinen zur Verfügung stehen und in denen ein besonderes Urteilsvermögen vonnöten ist, weil die entsprechende Situation neu ist oder ihr Eintritt in der Vergangenheit so unwahrscheinlich erschien, dass der Unternehmer sich nicht darauf vorbereitet hat. Als Beispiel nennt Casson das Auftreten einer neuen Technologie. ${ }^{321}$ In den Bereichen F\&E und Innovation treten permanent neue, noch nie dagewesene Situationen auf. Somit stellen diese Unternehmensfelder Kernbereiche dar, in denen die Urteilsfähigkeit des Unternehmers besonders gefragt ist.

Im Gegensatz zur Annahme der vollkommenen Information in der klassischen Volkswirtschaftslehre ist die Gewinnung und Verarbeitung von Informa-

317 Casson (2001), S. 528.

318 Casson (2003), S. 20.

319 Vgl. Casson (2001), S. 525.

320 Vgl. Casson (1992), S. 190.

321 Vgl. Casson (2005), S. $331 \mathrm{ff}$. und Casson (2001), S. $530 \mathrm{f}$. 
tionen in der Realität mit Transaktionskosten verbunden (vgl. Abschnitt 3.1.2). Wie von Hayek feststellte, kann der Unternehmer gar nicht alle für eine Entscheidung relevanten Informationen erhalten und verarbeiten. Die Erträge der Informationsbeschaffung müssen die Kosten aufwiegen. Zwar sind die Informations- und Kommunikationskosten im Laufe der Zeit deutlich gefallen, aber gleichzeitig hat die Fülle der verfügbaren Informationen zugenommen. Der Unternehmer muss sich nun in dieser Fülle zurechtfinden und die für ihn wichtigen Informationen herausfiltern. Deswegen ist davon auszugehen, dass diese Aufgabe für ihn trotz des bedeutenden technischen Fortschritts in der Informationsund Kommunikationstechnologie keinesfalls leichter geworden ist.

Der Unternehmer kann also keine vollkommene Information erlangen und fällt deshalb seine Entscheidungen unter Unsicherheit. Allerdings variiert das Ausmaß der Unsicherheit für verschiedene Personen. In diesem Zusammenhang ist die persönliche Nähe zum Geschehen von Bedeutung, ebenso wie die Zuverlässigkeit einer zur Verfügung stehenden Information. Ein Netzwerk von persönlichen Kontakten kann hier eine wichtige Rolle spielen, weil sie dem Unternehmer die Möglichkeit zur Erlangung vertraulicher Informationen eröffnet. Vor allem aber unterscheiden sich Entrepreneure von anderen Unternehmern durch ihre Interpretationsfähigkeit der vorhandenen Informationen und die daraus resultierende Qualität ihrer zu bewertenden Entscheidungen. ${ }^{322}$

Der Entrepreneur nach Casson ist ein Informationsmanager. Da er die Erfolgsaussichten eines Projektes aufgrund seiner besseren Informiertheit optimistischer einschätzt als seine Umwelt, ist es seine Aufgabe, relevante Gruppen wie Kapitalgeber und Angestellte durch optimistisches und selbstsicheres Auftreten vom zukünftigen Erfolg zu überzeugen. Gleichzeitig versucht er, potentielle Konkurrenten vom Markt fernzuhalten, indem er ihnen gegenüber derartige Projekte als nicht sehr erfolgsträchtig beschreibt. ${ }^{323}$

Des Weiteren ist der Entrepreneur bei Casson auch der „market-maker“3324. Ohne den Entrepreneur gäbe es überhaupt keine Märkte. Hierin stellt sich Casson stark gegen die neoklassische Theorie (vgl. Abschnitt 5.1), die die Hilfsfigur des Walrasianischen Auktionators als unabhängigen Preismittler mit vollständiger Information dazu benutzt, Angebot und Nachfrage in Übereinstimmung zu bringen. Der Markt ist der Ausgangspunkt der Neoklassik, auf dem die Anbieter ihre Güter und Dienstleistungen anbieten. Bei Casson dagegen schafft der Entrepreneur erst den Markt durch die Entwicklung neuer Produkte und Produktionsprozesse, die Abwandlung bereits existierender Produkte bzw. die Versorgung neuer Nachfragergruppen mit bereits existierenden Produkten. ${ }^{325}$

322 Vgl. Casson (2001), S. 528 f. und 536 f., ebenso wie Pratt und Zeckhauser (1985), S. 7.

323 Vgl. Casson (2005), S. $340 \mathrm{ff}$.

324 Casson (2005), S. 335.

325 Vgl. Casson (2005), S. 335 f. 


\subsubsection{Empirische Untersuchungen}

Kaish und Gilad (1991) finden in ihrer Untersuchung für New Jersey/USA heraus, dass sich das Informationsverhalten von Unternehmensgründern und in Großunternehmen angestellten Managern aus mittleren und höheren Führungsebenen unterscheidet. Unternehmensgründer und Manager unterscheiden sich nicht in ihrer Inanspruchnahme von üblichen Informationsquellen und Methoden der Informationssuche, wie z. B. Gespräche mit Angestellten, Kunden und Mitbewerbern. Allerdings schenken Unternehmensgründer sowohl den Medien als auch ungewöhnlichen Informationsquellen eine stärkere Beachtung. $\mathrm{Zu}$ Letzteren zählen beispielsweise Patentarchive und Gespräche mit fremden Mitreisenden in Zug oder Flugzeug. Auch beschäftigen sich Unternehmensgründer in ihrer Freizeit stärker mit für ihre Geschäftstätigkeit potentiell interessanten Inhalten und Informationsquellen und nutzen durch Familie und Freunde gewonnene Informationen für ihr Unternehmen. ${ }^{326}$ Die Autoren schließen daraus, dass Unternehmensgründer tatsächlich eine höhere „alertness“ im Kirzner'schen Sinne aufweisen als Manager und auch in ihrer Freizeit offen gegenüber Anregungen sind, die sich als nützlich für ihr Unternehmen erweisen können. Zwar ist die Abgrenzung des Unternehmers in dieser Studie nicht identisch mit der in dieser Arbeit verwendeten Abgrenzung, da in dieser Arbeit Manager aus höheren Führungsebenen ebenfalls als Unternehmer betrachtet werden. Dennoch ist das Ergebnis von Kaish und Gilad interessant und deutet darauf hin, dass Unternehmensgründer eher dem Kirzner'schen Entrepreneur entsprechen als angestellte Manager.

Mit der Frage des Ablaufs der unternehmerischen Entscheidungsfindung beschäftigen sich Busenitz und Barney (1997). Sie zeigen empirisch, dass Unternehmensgründer in ihrer Entscheidungsfindung stärker auf Entscheidungsheuristiken bzw. Kognitionen (vgl. Abschnitt 3.1.1.) zurückgreifen als in Großunternehmen angestellte Manager, denen mindestens zwei Funktionsbereiche unterstehen. ${ }^{327}$ Die unternehmerische Interpretation der vorhandenen Informationen kann somit schneller erfolgen als die Interpretation der Manager und auch eher zu besseren Entscheidungen führen. Auch wenn der von den Autoren angewendete Unternehmerbegriff sehr eng ist und deswegen wiederum vom in dieser Arbeit verwendeten Unternehmerbegriff abweicht, geben ihre Ergebnisse dennoch Aufschluss über mögliche Besonderheiten der unternehmerischen Entscheidungsfindung.

326 Vgl. Kaish und Gilad (1991), S. 51 ff.

327 Vgl. Busenitz und Barney (1997), S. 14 ff. 


\subsubsection{Anwendung der Theorie auf Innovation und Innovationsförderung}

Bei Anwendung der Erkenntnisse dieser Entrepreneurship-Theorieansätze auf die EO Innovation wird deutlich, dass innovativ tätige Unternehmer gut informiert über den aktuellen Stand der Forschung und die Innovationstätigkeit der Konkurrenz auf ihrem Gebiet sein müssen. Es stellt sich hier die Frage, ob anstelle des informierten Unternehmers, welcher das Analyseobjekt der Entrepreneurship-Theorie darstellt, nicht eher das informierte Unternehmen als Ganzes das für diese Arbeit relevante Analyseobjekt sein sollte. Denn die Informiertheit des Unternehmens hängt ja nicht ausschließlich vom Unternehmer $a b$, sondern z. B. auch von seinen Angestellten. Da der Unternehmer allerdings durch seine Persönlichkeit und die Art seiner Unternehmensführung die Unternehmenskultur stark beeinflusst, ${ }^{328}$ können Unternehmer und Unternehmen hier gleichgesetzt werden. Es ist davon auszugehen, dass Faktoren wie Innovationsfreundlichkeit und Offenheit des Unternehmens für Anregungen von außen stark durch den Unternehmer beeinflusst werden. Außerdem beweist der Unternehmer seine besonderen Fähigkeiten ja gerade auch durch die Auswahl seiner Mitarbeiter, wie schon durch Kirzner hervorgehoben wurde. Somit können von den Mitarbeitern und dem Unternehmen insgesamt Rückschlüsse auf den Unternehmer gezogen werden.

Schumpeter (1926) hat schon darauf hingewiesen, dass die unternehmerische Intuition aufgrund der steigenden Menge an verfügbaren Informationen im Laufe der Zeit immer unwichtiger wird. ${ }^{329}$ Stattdessen gewinnt die rationale, organisierte und kontinuierliche F\&E-Tätigkeit innerhalb des Unternehmens an Bedeutung. ${ }^{330}$ Die Ausprägung dieser Aktivität hängt von den absorptiven Fähigkeiten des Unternehmers und seiner Mitarbeiter ab, um beispielsweise Informationen über den neuesten technischen Fortschritt unverzüglich für die Innovationstätigkeit des Unternehmens zu nutzen ${ }^{331}$ : ,Purposeful, systematic innovation begins with the analysis of the opportunities. "332 Drucker (1985) nennt sieben Informationsquellen, aus denen Innovationsmöglichkeiten entspringen kön-

nen und die deswegen vom Unternehmer gründlich beobachtet werden sollen. ${ }^{333}$ Vier der Quellen sind innerhalb des Unternehmens bzw. des Wirtschaftszweiges zu finden: unerwartete Ereignisse, Unstimmigkeiten zwischen Realität und Idealzustand, Bedürfnisse der Produktionsweise und Veränderungen der Industrieoder der Marktstruktur. Drei weitere Quellen befinden sich außerhalb des Wirtschaftszweiges: demographische Veränderungen, Präferenzänderungen der Kon-

328 Vgl. Kets de Vries und Miller (1986), S. 267.

329 Vgl. Schumpeter (1926), S. 125.

330 Vgl. Langlois (2002), S. $18 \mathrm{ff}$.

331 Vgl. Cohen und Levinthal (1990), S. 138 ff.; Busom (2000), S. 118.

332 Drucker (1985), S. 134.

333 Vgl. Drucker (1985), S. 35. 
sumenten sowie neues Wissen jeglicher Form. Die absorptiven Fähigkeiten des Unternehmens bestimmen das Ausmaß, in dem diese Informationsquellen für die eigene Innovationstätigkeit genutzt werden können, und hängen wiederum von den in der Vergangenheit akkumulierten Informationen ab. ${ }^{334}$

Es gibt bislang nur wenige empirische Untersuchungen, die sich mit der Anwendung des in den vergangenen Abschnitten vorgestellten Teilbereichs der Entrepreneurship-Theorie auf die Innovativität von Unternehmen beschäftigen. Eine dieser wenigen Untersuchungen stammt von Lahner (2004) und hat ihren theoretischen Hintergrund nicht im Entrepreneurship-Bereich. Lahner findet in seiner Untersuchung von niedersächsischen Handwerksunternehmen heraus, dass innovative Handwerksunternehmen als wichtigste Quelle externen Wissens frei verfügbares Know-how ansehen. Die befragten Handwerksunternehmen führen ihre Innovationstätigkeit hauptsächlich mit Hilfe eigener Ressourcen durch und greifen nur fallweise auf externes Know-how zurück. Genutzt wird dieses hauptsächlich in Form von Fachzeitschriften, Internet, Messen, Forschungseinrichtungen, Technologietransferstellen, Arbeitskreisen, Austausch mit Mitbewerbern sowie Kunden und Lieferanten. Die befragten innovativen Handwerksunternehmen stufen die Suche nach und die Aufnahme von Informationen über neueste technologische Entwicklungen als höchst bedeutsam für ihre Wettbewerbsfähigkeit ein. ${ }^{335}$

In einer Befragung von Unternehmen des Verarbeitenden Gewerbes in den USA von Klevorick et al. (1995) gaben die befragten Unternehmen an, dass $\mathrm{Zu}$ lieferer, Kunden und Wettbewerber die wichtigsten Informationsquellen für technologisches Wissen seien. Staatliche Forschungseinrichtungen und unabhängige Erfinder besaßen dagegen deutlich weniger Relevanz. ${ }^{336}$ Eine Studie von Unger und Zagler (2003) untersuchte für elf europäische Staaten direkt die empirische Relevanz von verwendeten Informationen für die Innovationstätigkeit der Unternehmen des Verarbeitenden Gewerbes und des Dienstleistungssektors. Im Verarbeitenden Gewerbe haben insbesondere Informationen aus dem Unternehmen heraus, Unternehmensberatungen, Patentauskünfte und Messen einen positiven Einfluss auf die Innovationstätigkeit, während im Dienstleistungsbereich Informationen von Kunden, Tagungen und aus Zeitschriften besonders positiv auf die Innovationstätigkeit wirken. ${ }^{337}$

Diese empirischen Ergebnisse deuten allesamt darauf hin, dass innovativen Unternehmern die Wichtigkeit einer hohen Informationskompetenz im Sinne des idealtypischen Casson'schen Entrepreneurs bewusst ist. Allerdings können sie aufgrund der aufgeführten Informationsunvollkommenheiten nicht alle Informa-

334 Vgl. Cohen und Levinthal (1990), S. 129 und 141.

335 Vgl. Lahner (2004), S. 236 f. und 287.

336 Vgl. Klevorick et al. (1995), S. $198 \mathrm{ff}$.

337 Vgl. Unger und Zagler (2003), S. 303 f. 
tionsquellen nutzen, obwohl ihnen bewusst ist, dass sie durch eine intensivere Nutzung besser informiert wären.

Innovative Unternehmer scheinen also generell dem Casson'schen Entrepreneur für den Bereich Innovation zu entsprechen. Aus den vorgestellten Theorieansätzen können jedoch auch Aussagen getroffen werden, die sich auf diejenigen Unternehmer beziehen, die sich über Fördermöglichkeiten informieren und sich für Innovationsfördermittel bewerben. Der „Förderdschungel“" wurde bereits in Abschnitt 4.5.2 beschrieben. Die Vielzahl an existierenden Förderprogrammen unterschiedlicher Fördermittelgeber mit verschiedensten Themenspezialisierungen sowie die unterschiedlichsten privaten und öffentlichen Informationseinrichtungen führen dazu, dass der Unternehmer Schwierigkeiten hat, die für sein Unternehmen und seine Projekte relevanten Förderprogramme zu entdecken. Aufgrund der erheblichen Transaktionskosten der Informationssuche werden Unternehmer sich nicht umfassend über die Gesamtheit aller existierenden Förderprogramme informieren wollen. Es erstaunt nicht, dass eine Befragung deutscher KMU aus dem Jahr 1998 zu dem Ergebnis kommt, dass sich $75 \%$ der Unternehmer nicht gut informiert über das Gebiet der Wirtschaftsförderung fühlen. ${ }^{338}$

Verschieden ausgebildete Informationskompetenzen innerhalb der Gruppe der Unternehmer können nun eine mögliche Erklärung dafür darstellen, warum sich manche Unternehmer für Fördermittel bewerben und andere wiederum nicht. Dabei ist von der Voraussetzung auszugehen, dass geförderte Unternehmer tatsächlich besser informiert sind als nicht geförderte Unternehmer, was den Bereich Wirtschaftsförderung angeht. Denn dadurch, dass sie gefördert werden, zeigen geförderte Unternehmer, dass sie den bestehenden „Förderdschungel“" erfolgreich durchdrungen haben. Sie haben trotz der unübersichtlichen Situation ein für sie und ihr Unternehmen passendes Programm gefunden. Ob sich die Unternehmer nun aktiv über Förderprogramme informiert haben oder z. B. vom Innovationsberater einer Kammer auf die Existenz eines Förderprogramms hingewiesen wurden, führt zu derselben Schlussfolgerung: Geförderte Unternehmer haben die richtigen Informationsquellen benutzt, um ein für sie passendes Programm zu finden. Denn damit ein Unternehmer von den Beratern der Kammern direkt angesprochen wird, muss er sich in der Region profiliert haben, indem er beispielsweise in Netzwerken aktiv ist oder gute persönliche Kontakte zu den Kammern pflegt. Nun gibt es zwei mögliche Erklärungsansätze für das Zustandekommen der besseren Informiertheit der geförderten Unternehmer, die in diametral entgegengesetzten Hypothesen münden.

Erstens könnten die über eine besonders gut ausgeprägte Informationskompetenz verfügenden Unternehmer sowohl im Bereich Innovation als auch im Bereich Wirtschaftsförderung besser informiert sein. Denn ihnen könnte die Suche

338 Vgl. Förderer et al. (1998), S. 24. 
nach und die Verarbeitung von Informationen generell leichter fallen und sie könnten Synergieeffekte nutzen. Somit entsprechen sie eher dem idealtypischen Casson'schen Entrepreneur.

Hypothese 6: Unternehmer, die sich um Innovationsförderung bewerben, sind in den Bereichen Innovation und Wirtschaftsförderung besser informiert als Unternehmer, die sich nicht für Förderprogramme bewerben.

Zweitens könnte es jedoch der Fall sein, dass Unternehmer sich wegen der sie umgebenden Informationsflut in ihrer Informationssuche und -kompetenz auf bestimmte Bereiche beschränken. Diese Aussage steht in Einklang mit Casson, der darauf hinweist, dass die komparativen Vorteile von Unternehmern bezogen auf verschiedene Informationsarten differieren. ${ }^{339}$ So könnten sich manche Unternehmer auf die Suche nach Informationen über Nachfrageentwicklungen, die neuesten F\&E-Erkenntnisse und andere die Innovationstätigkeit beeinflussende Faktoren spezialisieren. Andere Unternehmer spezialisieren sich dagegen auf die Suche nach für sie in Frage kommende Förderprogramme und die damit verbundenen Bewerbungsformalitäten und entdecken deswegen häufiger die Förderprogramme, bei denen ihre Bewerbung Erfolg versprechend ist. Diese Argumentation könnte auch einen Erklärungsansatz dafür bieten, warum bestimmte Unternehmer sich auf die Bewerbung um Fördermittel aus den verschiedensten Programmen spezialisieren.

Hypothese 7 (entgegen Hypothese 6): Unternehmer, die sich um Innovationsförderung bewerben, sind im Bereich Wirtschaftsförderung besser informiert als Unternehmer, die sich nicht für Förderprogramme bewerben. Aber sie sind nicht besser informiert, was den Bereich Innovation angeht.

Neben der Informationskompetenz spielt noch eine zweite, damit verwandte Fähigkeit des Unternehmers bei seiner Bewerbung für ein Förderprogramm eine Rolle. Dabei handelt es sich um die Fähigkeit, bürokratische Anforderungen, wie Formulare auszufuillen, einen Förderantrag zu stellen und die Dokumentation der Fördermittelverwendung vorzunehmen, umsetzen zu können. Zeppernick drückt es folgendermaßen aus: „Prämiert wird in einem solchen System primär der ,staatliche Unternehmer;, also derjenige, der Anträge überzeugend begründen kann, das Angebot staatlicher Förderprogramme am besten kennt und es zu nutzen versteht, nicht hingegen der Pionierunternehmer, der sich dem Wettbewerb des Marktes stellt und durch Risikoübernahme und Innovation Pionierren-

339 Vgl. Casson und Wadeson (2007), S. 287. 
ten erzielt. “340 Es ist zu vermuten, dass die beschriebene Fähigkeit mit der Informationskompetenz des Unternehmers zusammenhängt, so dass ein Unternehmer, der komparative Vorteile in der Informationssuche und -verarbeitung besitzt, gleichzeitig auch gute Fähigkeiten im Ausfüllen vom Formularen und der überzeugenden Präsentation seiner Ideen hat.

Wenn diese Fähigkeit des Unternehmers nicht sehr ausgeprägt ist bzw. er der Überzeugung ist, dass der bürokratische Aufwand einer Bewerbung für ein Förderprogramm unangemessen hoch ist, wird er sich nicht für das Programm bewerben. Unternehmer haben auch im Rahmen ihrer gewöhnlichen Geschäftstätigkeit genügend organisatorischen Aufwand zu erledigen, so dass sie davon überzeugt sein müssen, dass der zusätzliche Aufwand durch den zusätzlichen Nutzen der eventuell zu erhaltenden Fördermittel aufgewogen wird. Somit ist es möglich, dass Unternehmer, die ein für sie relevantes Förderprogramm entdeckt haben (erste Phase der EO), sich deswegen nicht für dieses Programm bewerben, weil sie den mit einer Bewerbung verbundenen bürokratischen Aufwand als zu hoch ansehen. Falls der bürokratische Aufwand in der Realität nicht so hoch ist wie in ihrer Vorstellung, liegt unvollständige Information vor, die potentiell geeignete Unternehmer von einer Bewerbung abhalten könnte. Ob im Rahmen des NIFP eine derartige Informationsasymmetrie vorliegt, wird mit Hilfe folgender Hypothese getestet:

Hypothese 8: Der Bewerbungs- und Dokumentationsaufwand ist in der Realität nicht so groß wie in der Vorstellung derjenigen Unternehmer, die sich nicht für das NIFP beworben haben.

Bevor auch diese Hypothese in Kapitel 6 am Beispiel des NIFP auf seine empirische Evidenz getestet wird, beschäftigt sich der folgende Abschnitt mit der zweiten Phase der EO, der Bewertung der EO durch den Unternehmer.

\subsection{Zweite Phase: Bewertung der EO}

Nachdem der Unternehmer eine EO entdeckt hat, muss er sich entscheiden, ob er sie ausbeuten will oder nicht. Zu diesem Zweck nimmt er eine Bewertung der EO vor und trifft anschließend eine Entscheidung. Die Bewertung hängt zum einen von den Eigenschaften der EO und zum anderen von individuellen Präferenzen des Unternehmers ab. ${ }^{341}$ Bei der Bewertung der EO spielt insbesondere die Risikoeinstellung des Unternehmers eine entscheidende Rolle, weswegen im

340 Zeppernick (1985), S. 79.

341 Vgl. Shane und Venkataraman (2000), S. 222 f. 
Folgenden diejenigen Entrepreneurship-Theorieansätze vorgestellt werden, die den idealtypischen Entrepreneur als Träger besonderer Risiken definieren.

\subsubsection{Der Entrepreneur als Risikoträger nach Cantillon}

Cantillon (1755) ist einer der ersten Nationalökonomen, der der Figur des Unternehmers besondere Berücksichtigung schenkt und den Begriff des „Entrepreneurs" benutzt. Der zu den Vorläufern der Klassiker gehörende Cantillon differenziert zwischen den abhängig Beschäftigten mit sicherer Entlohnung (,,gens à gages certains") und den Unternehmern mit unsicherer Entlohnung (,gens à gages inconnus "). ${ }^{342}$ Die Entlohnung des Unternehmers unterliegt einem Risiko, da er nicht weiß, wie sich die Nachfrage entwickeln wird und zu welchem Preis er seine Waren absetzen kann. Damit er einen Anreiz hat, dieses Risiko zu tragen, erhält er den Unternehmergewinn bzw. Profit im Gegensatz zum Angestellten, der kein Risiko trägt und für seine Arbeitskraft eine fest vereinbarte Entlohnung bekommt. ${ }^{343}$

Eine ähnliche Definition des Entrepreneurs als Risikoträger findet sich bei Say (1861), der zwischen den Beschäftigten mit festem Einkommen und den Unternehmern mit ungewissem Unternehmerprofit unterscheidet. ${ }^{344}$ Der ebenfalls den Klassikern zuzuordnende Mill (1848) teilt den Profit des Unternehmers, den er als Kapitalisten bezeichnet, in drei Teile auf. ${ }^{345}$ Der erste Teil besteht aus dem Zins als Kompensation dafür, dass das Kapital nicht unmittelbar für Konsum aufgewendet wird. Der zweite Teil bildet die Entlohnung für die Arbeitskraft und Zeit, die der Unternehmer für die Überwachung der Produktion aufwendet. Der dritte Teil ist eine Entschädigung für das übernommene Risiko, da der Unternehmer den totalen oder zumindest teilweisen Verlust seines Kapitals riskiert: "The rate of profit greatly exceeds the rate of interest. The surplus is partly compensation for risk. By lending his capital, on unexceptionable security, he runs little or no risk. But if he embarks in business on his own account, he always exposes his capital to some, and in many cases to very great, danger of partial or total loss. For this danger he must be compensated, otherwise he will not incur it. "346

Von Thünen (1875) weist ebenfalls auf die Wichtigkeit der Ungewissheit für die Rechtfertigung des Unternehmergewinns hin. Der Unternehmer erhält den Unternehmergewinn als Kompensation für die Übernahme bestimmter Risiken, gegen die er sich nicht versichern kann: „Es gibt keine Assecuranzgesell-

342 Cantillon (1755), S. 71.

343 Ähnliche Definitionen finden sich auch bei anderen französischen Autoren des 18. Jahrhunderts wie Turgot und Quesnay, vgl. Kirzner (1979), S. 39.

344 Vgl. Say (1861), S. $346 \mathrm{f}$.

345 Vgl. Mill (1848), S. 405 f.

346 Mill (1848), S. 406. 
schaft gegen alle und jede Gefahr, die mit der Uebernahme eines Gewerbes verbunden ist; ein Theil der Gefahr muß immer von dem Unternehmer selbst getragen werden. Durch das bloße Sinken der Preise der Produkte, Fabrikate und Handelswaaren kann der Pächter eines Guts, der Fabrikant wie der Kaufmann, sein ganzes Vermögen verlieren - und gegen diese Gefahr gibt es keine Assecuranzgesellschaft.

Von Mangoldt (1855) rechtfertigt den Unternehmergewinn ebenfalls mit der Übernahme spezieller unternehmerischer Risiken. ${ }^{348}$ Der Gewinn besteht laut von Mangoldt aus drei Teilen: ,1) Entschädigung für die Last der Gefahr (Gefahrprämie). 2) Entschädigung für die dargebrachten Capitalnutzungen und Arbeitsleistungen (Unternehmerzins und-Lohn). 3) Vortheile, die aus der relativen Seltenheit der unternehmungsfähigen Subjecte fließen (Unternehmerrente). " ${ }^{349}$ Ebenso wie bei Mill und Cantillon entschädigt den Unternehmer also ein Teil des Profits für das übernommene Risiko, während ein anderer Teil die Arbeitsleistung des Unternehmers vergütet. Der dritte Teil des Unternehmerprofits ist eine „Seltenheitsprämie“"350 für die wenigen Wirtschaftssubjekte, die als Unternehmer arbeiten wollen und können. Von Mangoldt weist explizit auf das geringe Angebot an Unternehmern hin, das sich erstens durch gesetzliche Vorschriften und kulturelle Faktoren, welche die Unternehmerzahl beschränken, erklären lässt. Zweitens führt er aus, „daß es thatsächlich an zu Unternehmern geeigneten Persönlichkeiten gebricht, indem die natürlichen Bedingungen für die Unternehmung eines bestimmten Geschäfts sich nur in einer gewissen beschränkten Anzahl von Personen vorfinden “351.

Viele moderne Vertreter der Entrepreneurship-Theorie teilen von Mangoldts Ansicht nicht, dass nur ein geringer Bevölkerungsteil aufgrund der Ausprägung bestimmter Persönlichkeitseigenschaften dazu geeignet ist, unternehmerisch tätig zu werden. Stattdessen wird der „entrepreneurship education“, der Unterrichtung von für Gründung und Führung eines Unternehmens wichtigen Fähigkeiten an Schule und Universität, ein großer Stellenwert eingeräumt. Ziel des Unterrichts ist eine Erhöhung der Zahl der erfolgreichen Unternehmensgründungen. ${ }^{352}$

\subsubsection{Unterscheidung zwischen Risiko und Unsicherheit nach Knight}

In der Tradition von Cantillon betont Knight (1921) die Thematik des Neuen und des Wandels im Wirtschaftsleben, auf die der Unternehmer reagieren muss.

347 von Thünen (1875), S. 84.

348 von Mangoldt (1855), S. 36.

349 von Mangoldt (1855), S. 81.

350 von Mangoldt (1855), S. 110.

351 von Mangoldt (1855), S. 115

352 Vgl. z. B. Schulte (2007). 
Knight unterscheidet wie von Thünen zwischen versicherbarem Risiko (,risk") und Unsicherheit (,uncertainty"). Wenn die Wahrscheinlichkeiten eines Ereignisses bekannt sind, handelt es sich um ein versicherbares Risiko. Bei Unsicherheit sind die versicherbaren Wahrscheinlichkeiten unbekannt, so dass keine Versicherung möglich ist. ${ }^{353}$

Knights Theorieansatz beschäftigt sich damit, wie ein Unternehmer auf Unsicherheit reagiert und mit den damit zusammenhängenden komplexen und sich ständig wandelnden ökonomischen Rahmenbedingungen umgeht. Denn bevor die Nachfrage beobachtet werden kann, muss die Produktion erfolgen. Der Unternehmer muss Prognosen über den zukünftigen Preis und die nachgefragte Menge des Endproduktes sowie über die Produktivität der eingesetzten Produktionsfaktoren erstellen. ${ }^{354}$ Wie bei Cantillon ist der Profit bei Knight eine Prämie für die Risikoübernahme der nicht zu versichernden Unsicherheit. ${ }^{355}$ Außerdem dient der Profit als Kompensation für die Marktprognosen und Schätzungen, die der Unternehmer anstellen muss: „But the facts upon which the working-out of the organization depends can no longer be objectively determined with accuracy by experiment; all the data in the case must be estimated, subject to a larger or smaller margin of error [...]. The function of making these estimates and of ,guarenteeing 'their value to the other participating members of the group falls to the responsible entrepreneur in each establishment, producing a new type of activity and a new type of income entirely unknown in a society where uncertainty is absent. "356 Der Unternehmerprofit ist das Residuum nach Abzug der festen Entlohnungen der anderen Produktionsfaktoren vom Umsatz. Der Unternehmer erhält auch eine Entlohnung für seine Koordinationsaufgaben (vgl. Abschnitt 5.2.1), doch stellen diese für Knight nicht die entscheidenden Aufgaben des Unternehmers dar. ${ }^{357}$

Der Unternehmer übernimmt zwei voneinander untrennbare Funktionen: Verantwortung für seine Entscheidungen und Kontrolle der Produktion. ${ }^{358}$ Ein erfolgreicher Knight'scher Entrepreneur trifft unter struktureller Unsicherheit Entscheidungen, die sich ex post als erfolgreich herausstellen. Dafür erhält er einen Gewinn. Die schon von Casson und von Mises erwähnte Bewertungsfähigkeit der unsicheren Rahmenbedingungen (vgl. Abschnitte 5.4.2 und 5.4.3) im Zusammenspiel mit den Entscheidungen und Bewertungen seiner Konkurrenten bestimmen seinen unternehmerischen Erfolg. ${ }^{359}$

353 Vgl. Knight (1921), S. 268.

354 Vgl. Knight (1921), S. $268 \mathrm{ff}$.

355 Vgl. zu dieser Einschätzung Casson (2001), S. 526.

356 Vgl. Knight (1921), S. 276.

357 Vgl. Knight (1921), S. 277 ff.

358 Vgl. Knight (1921), S. 271.

359 Vgl. Knight (1921), S. 280 f. 


\subsubsection{Moderne Theorieansätze}

In der modernen Literatur finden sich einige Theorieansätze, die auf den Erkenntnissen Knights aufbauen und sich mit der speziellen Ausprägung der Risikoneigung als einer besonderen Eigenschaft des Entrepreneurs beschäftigen. Zu den einflussreichsten Ansätzen zählt das neoklassische Modell von Kihlstrom und Laffont (1979). In diesem Modell unterscheiden sich Individuen im Grad ihrer Risikoaversion, wobei es einen Grenzwert für die Risikoaversion gibt. Individuen mit einer geringeren Risikoaversion werden Unternehmer, während Individuen mit einer höheren Risikoaversion eine angestellte Beschäftigung anstreben. ${ }^{360}$

Eine Erklärung für die Tatsache, dass sich bestimmte Wirtschaftssubjekte risikofreudig verhalten, ein Unternehmen gründen und fortan als Unternehmer tätig sind, liefert die Anwendung der ,prospect theory“ auf die Entrepreneurship-Theorie. Die ,prospect theory“ besagt, dass monetäre Verluste einen Nutzenverlust bewirken, der stärker ist als der Nutzengewinn, der durch einen monetären Gewinn in gleicher Höhe erzeugt wird. ${ }^{361}$ Ein angestelltes Wirtschaftssubjekt stellt nun eine Nutzenkalkulation an, um zu entscheiden, ob es unternehmerisch tätig werden oder angestellt bleiben will. $\mathrm{Zu}$ diesem Zweck vergleicht das Wirtschaftssubjekt die möglichen unsicheren Gewinne durch eine unternehmerische Tätigkeit mit dem Status quo, der im Vergleich zum erwarteten Gewinn des Unternehmerdaseins mit einem sicheren monetären Verlust verbunden ist. Wenn sich nun Wirtschaftssubjekte mit einer unternehmerischen Veranlagung auf die Analyse der Verluste konzentrieren, ergibt sich bei Anwendung der „prospect theory“ die Schlussfolgerung, dass sich diese Wirtschaftssubjekte risikofreudig verhalten und eine unternehmerische Tätigkeit aufnehmen, um den sicheren Verlusten durch Beibehaltung der angestellten Tätigkeit $\mathrm{zu}$ entgehen. ${ }^{362}$

\subsubsection{Empirische Untersuchungen}

Die empirischen Ergebnisse bzgl. einer besonderen Risikoneigung von Unternehmern sind nicht eindeutig und lassen verschiedene Interpretationsmöglichkeiten zu.

Die Meta-Analyse von Stewart Jr. und Roth (2001) fasst die Ergebnisse von 14 empirischen Untersuchungen aus den Jahren 1980-1999 zusammen und kommt $\mathrm{zu}$ dem Ergebnis, dass Unternehmer eine deutlich höhere Risikobereitschaft haben als angestellte Manager. ${ }^{363}$ Van Praag und Cramer (2001) untersu-

360 Vgl. Kihlstrom und Laffont (1979), S. 722 ff.; Bianchi und Henrekson (2005), S. 360 ff.

361 Vgl. Tversky und Kahneman (1986), S. S257 ff.

362 Vgl. Baron (2004), S. 224 f.

363 Vgl. Stewart Jr. und Roth (2001), S. 150. 
chen die ökonomische Risikoeinstellung von Unternehmern im Vergleich zu anderen Bevölkerungsgruppen in den Niederlanden. Die Autoren finden ebenfalls empirische Belege für eine deutlich geringere Risikoaversion der Unternehmer. ${ }^{364}$ Auch die Ergebnisse der empirischen Untersuchung von Caliendo et al. (2008) unterstützen die Erkenntnisse der Entrepreneurship-Theorie bezogen auf die Risikoeinstellung der Unternehmer. Die Autoren untersuchen die Risikoeinstellungen von deutschen Unternehmensgründern mit Hilfe der Erhebungen des Sozioökonomischen Panels von 2004 und 2005. Laut dieser Untersuchung besitzen Unternehmensgründer - und somit auch die späteren Unternehmer eine niedrigere Risikoaversion als andere Wirtschaftssubjekte. Allerdings trifft dieses Ergebnis nur für diejenigen Unternehmensgründer zu, die vorher angestellt waren. Diejenigen, die vor ihrer Unternehmensgründung arbeitslos oder inaktiv waren, unterscheiden sich in ihrer Risikoeinstellung nicht signifikant vom Rest der Bevölkerung. ${ }^{365}$

Die Zusammenfassung von 14 Studien aus dem Zeitraum 1987-2002 im Rahmen der Meta-Analyse von Miner und Raju (2004) findet dagegen empirische Belege für eine geringere Risikobereitschaft der Unternehmer im Vergleich zur Kontrollgruppe angestellter Manager, ein Ergebnis, das den oben beschriebenen Hypothesen der Entrepreneurship-Theorie also vollkommen widerspricht. Die Autoren kombinieren ihre Ergebnisse mit der Studie von Stewart Jr. und Roth (2001) und kommen zu dem Ergebnis, dass es bislang keine zweifelsfreien empirischen Belege dafür gibt, dass sich die Risikobereitschaft der Unternehmer von der anderer Bevölkerungsgruppen unterscheidet. ${ }^{366}$

Auch Palich und Bagby (1995) finden keine empirischen Belege für Unterschiede in der Risikoeinstellung zwischen Unternehmern und angestellten Managern. Stattdessen zeigen sie theoretisch und empirisch Unterschiede in kognitiven Prozessen auf. Unternehmer reagierten auf die ihnen vorgelegten Fallbeispiele anders als die Vergleichsgruppe. So bewerteten die Unternehmer die Stärken und Chancen positiver und sahen Schwächen und Gefahren als weniger wichtig an. ${ }^{367}$ Daraus schließen die Autoren, dass nicht die Risikoeinstellung für die Abgrenzung der Unternehmer von anderen Wirtschaftssubjekten entscheidend ist, sondern dass Unternehmer eine Situation als „opportunity“ ansehen und sich auf die damit verbundenen Chancen konzentrieren. ${ }^{368}$ Anstelle von Risikoneigung wären somit Aufmerksamkeit und Urteilsfähigkeit des Unternehmers in Anlehnung an die Erkenntnisse aus Abschnitt 5.4 die entscheidenden Fähigkeiten zur Abgrenzung von Unternehmern und anderen Wirtschaftssubjekten.

364 Vgl. van Praag und Cramer (2001), S. 57 f.

365 Vgl. Caliendo et al. (2008).

366 Vgl. Miner und Raju (2004), S. 8 f.

367 Vgl. Palich und Bagby (1995), S. 432 ff.

368 Vgl. dazu auch Baron (2004), S. 224. 
Zu einem ähnlichen Ergebnis kommen Sarasvathy et al. (1998), die ein Quasi-Experiment mit Unternehmern und angestellten Managern durchführen, denen verschiedene mit Risiko behaftete Szenarien vorgelegt werden. Untersucht wurden die kognitiven Prozesse der Probanden in Bezug auf den Umgang mit Situationen, welche mit Risiko behaftet sind. Die Unternehmer benutzten den ungünstigsten Fall als Ausgangspunkt ihrer Betrachtung. Sie akzeptierten gegebene Risiken und versuchten Wege zu finden, die damit verbundenen Erträge zu erhöhen. Auch in dieser Untersuchung stellen also die Chancen eines Projektes für die Unternehmer die entscheidende Größe dar. Dagegen konzentrierten sich die Manager auf die höchsten zu erreichenden Erträge und versuchten, die damit verbundenen Risiken zu verringern. ${ }^{369}$

Der Einfluss des Reichtums auf die Risikoaversion eines Individuums und seine Berufswahl ist in der Literatur umstritten. Zwar ist die Argumentation schlüssig, dass arme Individuen eine geringere Risikoaversion haben könnten als reiche Individuen, weil die ärmeren Individuen weniger zu verlieren haben. Allerdings lassen sich auch gute Gründe für den umgekehrten Fall finden: Reichere Individuen könnten weniger risikoavers sein, weil sie es sich eher leisten können, mit einem Projekt bzw. Unternehmen Verluste zu machen. ${ }^{370}$ Letztere Argumentation wird gestützt durch die Ergebnisse von Evans und Jovanovic (1989), die empirische Belege dafür liefern, dass Unternehmer unter Liquiditätsbeschränkungen leiden. Der Kapitalstock des Unternehmens beträgt nach Schätzungen der Autoren im Schnitt das Anderthalbfache des persönlichen Vermögens. Die Ausübung einer unternehmerischen Tätigkeit hängt somit entscheidend vom Vorhandensein des persönlichen Vermögens ab. ${ }^{371}$ Die an ihre Arbeit anschließenden Untersuchungen von Blanchflower und Oswald (1998) und von Holtz-Eakin et al. (1994) ergeben, dass der Erhalt einer Erbschaft die Entscheidung eines Wirtschaftssubjekts für die Gründung eines Unternehmens positiv beeinflusst. ${ }^{372}$

\subsubsection{Anwendung der Theorie auf Innovation und Innovationsförderung}

Die im vorigen Abschnitt vorgestellten Studien finden teilweise eine empirische Evidenz für Unterschiede in der Risikoeinstellung zwischen Unternehmern und anderen Bevölkerungsgruppen. Im Rahmen dieser Arbeit ist jedoch eine Differenzierung innerhalb der Gruppe der Unternehmer in Bezug auf ihre Risikoeinstellung vorzunehmen. Es wird untersucht, inwiefern Unterschiede in der Risikoeinstellung der Unternehmer erklären können, warum Unternehmer innovativ

369 Vgl. Sarasvathy et al. (1998), S. 212 f.

370 Vgl. Kihlstrom und Laffont (1979), S. 746 f.; Blanchflower und Oswald (1998), S. 42.

371 Vgl. Evans und Jovanovic (1989), S. 825.

372 Vgl. Blanchflower und Oswald (1998); Holtz-Eakin et al. (1994). 
tätig werden und warum Unternehmer sich um Innovationsfördermittel bewerben.

Stewart Jr. und Roth (2001) finden empirische Belege dafür, dass Unternehmer, deren Hauptinteresse auf einem ausgeprägten Unternehmenswachstum liegt, eine höhere Risikobereitschaft besitzen als Unternehmer, die eher an einem kontinuierlichen Einkommensstrom interessiert sind. ${ }^{373}$ Die EO Innovation beinhaltet erhebliche Wachstumschancen für das Unternehmen, da Innovationen die Wettbewerbsfähigkeit des Unternehmens erhöhen. So liefert beispielsweise eine Studie des Zentrums für Europäische Wirtschaftsforschung von 2006 empirische Evidenz für einen positiven Zusammenhang zwischen Innovativität und Wachstum bei Hightech-Unternehmensgründungen in Deutschland. Unternehmen, die kontinuierlich F\&E durchführen, weisen ein höheres Beschäftigungswachstum als weniger forschungsorientierte Unternehmen auf. Unternehmen, die selbst entwickelte neue Produktionsverfahren einsetzen oder eigene Patente nutzen, gehören sowohl hinsichtlich Umsatz als auch Beschäftigung eher zu den wachstumsstarken Unternehmen. ${ }^{374}$ Auch die Studie von Morone und Testa (2008) findet empirische Evidenz für einen positiven Zusammenhang zwischen Innovativität und Umsatzwachstum, wobei hier ein Datensatz von italienischen KMU zur Untersuchung herangezogen wird. ${ }^{375}$

Diese Ergebnisse legen die Schlussfolgerung nahe, dass Unternehmer mit einer relativ hohen Risikobereitschaft, die an einem positiven Unternehmenswachstum interessiert sind, die EO Innovation eher positiv beurteilen und durchführen - trotz der mit jeder Innovation einhergehenden Technologie- und Marktrisiken (vgl. dazu ebenfalls Abschnitt 2.3.3). Zusammenfassend würde eine hohe Innovationstätigkeit somit eher von risikofreudigen Unternehmern als von risikoscheuen durchgeführt werden.

Drucker (1985) hält allerdings seine praxisnahen Beobachtungen und die Erfahrungen erfolgreicher Unternehmer dagegen. Die Bedeutung des Risikos der Innovationstätigkeit würde in manchen Bereichen der EntrepreneurshipLiteratur überschätzt: „Of course innovation is risky. But so is stepping into the car to drive to the supermarket for a loaf of bread. All economic activity is by definition , high-risk: "376 Laut Drucker sind erfolgreiche, innovative Unternehmer nicht unbedingt risikofreudige Menschen. Stattdessen analysieren sie die mit einer Innovationsmöglichkeit zusammenhängenden Risiken und Chancen sorgfältig und versuchen, die Risiken zu minimieren: „, Successful innovators are conservative. They have to be. They are not, risk-focused'; they are, opportunity-focused: " 377 Wiederum findet sich der Anknüpfungspunkt bei den beson-

373 Vgl. Stewart Jr. und Roth (2001), S. 150.

374 Vgl. Niefert et al. (2006), S. 49 ff.

375 Vgl. Morone und Testa (2008), S. $322 \mathrm{ff}$.

376 Drucker (1985), S. 139.

377 Drucker (1985), S. 140. 
deren Informationsverarbeitungs- und Urteilsfähigkeiten, die Entrepreneure von anderen Unternehmern abgrenzen (vgl. Abschnitt 5.4).

Norton Jr. und Moore (2002) halten die Annahme ebenfalls für überholt, dass sich innovativ tätige Unternehmer durch eine besonders niedrige Risikoaversion gegenüber nicht innovativ tätigen Unternehmern auszeichnen. Stattdessen sei die in Abschnitt 5.4.2 beschriebene „alertness“ entscheidend für die Differenzierung zwischen innovativ und nicht innovativ tätigen Unternehmern. Bedingt durch in der Vergangenheit gemachte Erfahrungen und voneinander abweichenden Informationsständen entscheiden sich manche Unternehmer für die Durchführung von riskanten Projekten, während sich andere dagegen entscheiden. Die Entscheidung hängt jedoch nicht von unterschiedlichen Risikoeinstellungen, sondern von individuell unterschiedlichen Bayes'schen Wahrscheinlichkeiten $\mathrm{ab}$, also den von persönlichen Informationen und Einschätzungen beeinflussten Risikowahrnehmungen. ${ }^{378}$

Auch bei Betrachtung der gesamten unternehmerischen Tätigkeit ohne Beschränkung auf die Innovationstätigkeit vermutet Baron (2004), dass erfolgreiche Unternehmer fähiger sind, die mit einer Strategie verbundenen Risiken abzuschätzen und infolgedessen bessere Entscheidungen treffen. Laut dieser Argumentation wirken erfolgreiche Unternehmer eher risikoscheu. Sie entscheiden sich aber für risikoreiche Projekte, wenn die Abwägung der Chancen und Möglichkeiten und der zu erzielenden ökonomischen Ergebnisse für das Unternehmen positiv ausfällt. ${ }^{379}$

Sowohl Theorie als auch Empirie liefern insgesamt also keine eindeutigen Hinweise auf einen systematischen Unterschied in der Risikoeinstellung von innovativen und nicht innovativen Unternehmern, so dass folgende Hypothese aufgestellt werden kann:

\section{Hypothese 9: Innovative und nicht innovative Unternehmer unter- scheiden sich nicht in ihrer Risikoeinstellung.}

Bezogen auf die EO Innovationsförderung wurde bereits in Abschnitt 2.3.3 ausgeführt, dass Politiker mit Hilfe von Innovationsförderung Unternehmer darin unterstützen möchten, ihre Innovationstätigkeit zu erhöhen und damit zusammenhängend mehr Innovationsrisiken zu übernehmen. Für eher risikoscheue Unternehmer kann diese Förderung sinnvoll sein, weil sie mit Hilfe der Förderung risikoreiche Innovationsprojekte durchführen, die sie ohne diesen Anschub nicht durchgeführt hätten. Aus diesen Innovationsprojekten könnten von Politikern erhoffte positive Wachstums- und Beschäftigungseffekte für die Volkswirtschaft entstehen. Es ist zu vermuten, dass eher risikoscheue Unternehmer den

$378 \mathrm{Vgl}$. Norton Jr. und Moore (2002), S. $282 \mathrm{f}$.

379 Vgl. Baron (2004), S. 233. Vgl. auch Miner und Raju (2004), S. 10, und Gifford (2005), S. 45 ff., mit einer ähnlichen Argumentation. 
Aufwand, der bei einer Bewerbung für ein Innovationsförderprogramm und der Dokumentation der Fördermittelverwendung anfällt, in Kauf nehmen, um ihr finanzielles Risiko durch den Erhalt von Fördermitteln verringern zu können. Es könnte eine Art Selbstselektion erfolgen. Risikoscheue Unternehmer, die Innovationsprojekte durchführen wollen, informieren sich über die Verfügbarkeit von Innovationsförderprogrammen, bewerben sich eher für diese Programme und nehmen den damit verbundenen Aufwand in Kauf. Risikofreudige Unternehmer suchen sich dagegen eher andere Finanzierungsquellen für ihre Innovationsideen. Daraus ergibt sich folgende Hypothese:

Hypothese 10: Unternehmer, die sich um Innovationsförderung bewerben, sind risikoscheuer als Unternehmer, die sich nicht bewerben.

\subsection{Dritte Phase: Ausbeutung der EO}

Nach der Entdeckung der EO und ihrer Bewertung bzw. der Entscheidung über ihre Ausbeutung erfolgt die dritte Phase der EO: die Ausbeutung selbst. Bezogen auf Innovationen besteht die dritte Phase der EO in der Tätigung von Investitionen im Innovationsbereich durch den Unternehmer. Angewendet auf Innovationsförderung besteht die dritte Phase der EO darin, dass sich der Unternehmer für ein bestimmtes Innovationsförderprogramm bewirbt. Der in den folgenden Abschnitten vorgestellten Ansatz der Entrepreneurship-Theorie charakterisiert den idealtypischen Unternehmer - den Entrepreneur - über die erste Handlungsweise, die Ausnutzung der EO Innovation, indem ,the entrepreneur always searches for change, responds to it, and exploits it as an opportunity" 380. Der bis heute einflussreichste Vertreter dieses Theorieansatzes ist Schumpeter, dessen Ideen in den Abschnitten 5.6.2 und 5.6.3 vorgestellt werden. Zunächst widmet sich Abschnitt 5.6.1 jedoch den Vorarbeiten der Historischen Schule und von Thünens.

\subsubsection{Vorarbeiten: von Thünen und die Historische Schule}

Schon von Thünen erwähnt neben der Risikoübernahmefähigkeit des Unternehmers (vgl. Abschnitt 5.5.1) auch seine Innovatoreneigenschaft. Da für den Unternehmer im Gegensatz zum Angestellten sein eigenes Vermögen und seine Reputation mit seinem Geschäftserfolg verknüpft sind, erbringt er höhere Leistungen als der Angestellte bei gleichen Fähigkeiten. Insbesondere bei schlecht gehenden Geschäften wird die Kreativität des Unternehmers geweckt, der auch nachts über eine Verbesserung seiner Situation nachdenkt: „Aber die schlaflosen

380 Drucker (1985), S. 28. 
Nächte des Unternehmers sind nicht unproduktiv. Hier fasst er Pläne und kommt auf Gedanken zur Abwendung seines Missgeschicks, die dem besoldeten Administrator [so bezeichnet von Thünen den Angestellten], wie ernstlich derselbe auch seine Pflicht zu erfüllen streben mag, doch verborgen bleiben - weil sie erst aus der höchsten Anspannung aller auf einen Punkt gerichteten Geisteskräfte hervorgehen. Die Noth ist die Mutter der Erfindungen, und so wird auch der Unternehmer durch seine Bedrängniß zum Erfinder und Entdecker in seiner Sphäre. " ${ }^{381}$ Aus diesem Grund steht dem Unternehmer im Gegensatz zum angestellten Administrator eine zusätzliche Vergütung zu, die von Thünen „Industriebelohnung " nennt. ${ }^{382}$

Neben von Thünen finden sich erste Ansätze, den Unternehmer als Innovator zu betrachten, bei Vertretern der Historischen Schule der deutschen Nationalökonomie, auf deren heroischem Unternehmerbild Schumpeter aufbaut. So sah die Historische Schule den Unternehmer als einen besonderen Menschentyp an, der sowohl einen außerordentlich scharfen Verstand als auch eine besonders ausgeprägte Vorstellungskraft hat. Sein Ehrgeiz treibt ihn dazu an, die überkommenen Pfade der Volkswirtschaft zu verlassen und neue Unternehmungen zu wagen. Seine Rolle als Initiator wirkt derart, dass erst durch sein Eingreifen eine sinnvolle Produktion beginnt. Der Unternehmer treibt die Volkswirtschaft erst an, ohne ihn würde die Volkswirtschaft im Stillstand verharren. ${ }^{383}$

\subsubsection{Die Durchsetzung neuer Kombinationen}

Schumpeter (1926) bezieht sich mit seinem Idealbild des Unternehmers auf die Vorarbeiten der Historischen Schule. Wirtschaftswachstum entsteht bei Schumpeter durch grundlegende Innovationen, die er als „Durchsetzung neuer Kombinationen " bezeichnet (vgl. Abschnitt 2.1). Ein Entrepreneur muss nicht zwangsläufig der Erfinder der diesen Innovationen zugrunde liegenden Inventionen sein, er muss nur die Ressourcen einsetzen, um die Neuerungen als Innovationen umzusetzen. Auch muss der Entrepreneur nicht der Kapitalgeber sein. Seine Aufgabe besteht im Treffen von strategischen Entscheidungen und der Koordinierung des Faktoreinsatzes (vgl. Abschnitt 5.2.1). ${ }^{384}$

Beim Treffen dieser strategischen Entscheidungen kann auch der Entrepreneur nicht alle Auswirkungen seiner Entscheidungen im Voraus kalkulieren und in seine Entscheidungsfindung einbeziehen: „Hier kommt für den Erfolg alles auf ,Blick' an, auf die Fähigkeit die Dinge in einer Weise zu sehen, die sich dann hinterher bewährt, auch wenn sie im Momente nicht zu begründen ist, und das Wesentliche fest und das Unwesentliche gar nicht auffaßt, auch wenn und

381 von Thünen (1875), S. $86 \mathrm{f}$.

382 Vgl. von Thünen (1875), S. 87.

383 Vgl. Berghoff (2004), S. 35.

$384 \mathrm{Vgl}$. Schumpeter (1926), S. $129 \mathrm{ff}$. 
gerade dann, wenn man sich über die Grundsätze, nach denen man dabei verfährt, keine Rechenschaft geben kann. "385 Der Entrepreneur trifft seine Entscheidungen, da er sich nicht alle notwendigen Informationen aneignen und diese verarbeiten kann, notwendigerweise mit einem Bruchteil der verfügbaren Informationen. Hier finden sich Anknüpfungspunkte zur „bounded rationality“ von Simon (vgl. Abschnitt 3.1.1). Denn Schumpeter beschreibt letztlich genau diese: die begrenzte Rationalität des Unternehmers, der seine Entscheidungen unter Unsicherheit trifft und gezwungenermaßen eine Strategie des „satisficing“ verfolgt, weil er keine vollständige Information erlangen kann. ${ }^{386}$

\subsubsection{Der Schumpeter'sche Entrepreneur und Wirtschaftswachstum}

Bahnbrechende Innovationen führen zu starkem Wirtschaftswachstum und verändern sowohl die Struktur der Volkswirtschaft als auch die Allokation der Produktionsfaktoren grundlegend: „Die Durchsetzung neuer Kombinationen bedeutet also [...] Andersverwendung des Produktionsmittelvorrates der Volkswirtschaft. " 387 Diese Innovationen lösen nach der Theorie der langen Wellen langfristige Wachstumszyklen aus. ${ }^{388}$ Der Schumpeter'sche Entrepreneur, der diese Innovationen umsetzt, sorgt neben einem Wachstumsschub für die innovierenden Branchen gleichzeitig für einen Schrumpfungsprozess derjenigen Branchen und Unternehmen, die mit überkommenen Technologien arbeiten. Dieser Prozess wird auch als schöpferische Zerstörung bezeichnet.

Laut Schumpeter werden grundlegende Innovationen oft von Außenseitern getätigt, während die schon im Markt etablierten Unternehmer dem Fortschritt hinterherhinken: „, es waren [...] im allgemeinen nicht die Postmeister, welche die Eisenbahnen gründeten. "389 Jeder Unternehmer, der nach einer gewissen Zeit des Entrepreneur-Daseins nur noch reine Routinearbeit leistet, wird in dieser Phase von Schumpeter als „Wirt“ bezeichnet. Denn niemand kann so viel Kraft und Energie aufbringen, um dauerhaft ein Entrepreneur im Sinne Schumpeters zu sein - also einer, der die Volkswirtschaft grundlegend verändert. ${ }^{390}$ An dieser Stelle werden wiederum Parallelen zur Theorie des „satisficing“ (vgl. Abschnitt 3.1.1) deutlich. Nach der Theorie des ,satisficing“" strebt der Unternehmer nicht nach Gewinnmaximierung, sondern nur nach einem zufrieden stellenden Gewinn, der seinen Erwartungen entspricht. ${ }^{391}$ Erreicht der Unternehmer aber schon durch seine normale Geschäftstätigkeit einen zufrieden stellenden

385 Schumpeter (1926), S. 125.

386 Vgl. Langlois (2002), S. 19.

387 Schumpeter (1926), S. 103.

388 Vgl. Kondratieff (1926).

389 Schumpeter (1926), S. 101.

$390 \mathrm{Vgl}$. Schumpeter (1926), S. $110 \mathrm{ff}$.

391 Dafür gibt es auch zahlreiche empirische Belege, vgl. Simon (1959), S. 262. 
Gewinn, so besteht für ihn kein Anreiz, sich um risikoreiche Innovationen zu bemühen.

Doch Schumpeter weist nicht nur auf die Bedeutung des Entrepreneurs für Innovation und Wirtschaftswachstum hin. Er erwähnt gleichfalls, dass das Unternehmertum seine entscheidende Rolle bei Innovationen und damit zusammenhängend bezogen auf das Wirtschaftswachstum im Laufe der Zeit verliert. Denn dadurch, dass die verfügbaren Informationen in unserer Gesellschaft immer weiter steigen, verliert die Intuition des Unternehmers an Bedeutung: „Je genauer wir die natürliche und die soziale Welt kennen lernen, je vollkommener unsere Herrschaft über die Tatsachen wird, je größer mit der Zeit und fortschreitender Rationalisierung der Bereich wird, innerhalb dessen die Dinge einfach ausgerechnet, und zwar schnell und verläßlich ausgerechnet werden können, desto mehr tritt die Bedeutung gerade dieser Aufgabe [dem im vorigen Abschnitt beschriebenen „Blick"] zurück und muß deshalb die Bedeutung des Typus ,Unternehmer' [...] sinken “392. Dagegen bekommt die organisierte Forschungs- und Entwicklungstätigkeit in Großunternehmen einen größeren Stellenwert als die Kreativität des einzelnen Unternehmers, worauf Schumpeter in seinem Spätwerk verstärkt eingeht. ${ }^{393}$ Bedingt durch den kumulativen Charakter der F\&E-Tätigkeit werden hohe Eintrittsschranken auf dem Markt aufgebaut, so dass sich ein stabiles Oligopol von innovierenden Großunternehmen entwickelt. ${ }^{394}$ In der neueren Literatur überwiegt mittlerweile allerdings die Auffassung, dass sich die Innovationsaktivitäten von unabhängigen Unternehmern bzw. Erfindern und von F\&E-Abteilungen der Großunternehmen ergänzen und beide bis heute ihren eigenen nicht zu unterschätzenden Stellenwert im Innovationsprozess haben. Während die radikalen Innovationen und Ideen überproportional oft von kleinen unabhängigen Unternehmern und Erfindern kommen, liegt der Schwerpunkt der F\&E-Aktivität der Großunternehmen bei inkrementellen Innovationen, der schrittweisen Weiterführung und Verbesserung von Produkten und Produktionsprozessen. ${ }^{395}$

Bezogen auf die Rolle des Unternehmers im volkswirtschaftlichen Wachstumsprozess unterscheidet sich der Schumpeter'sche Entrepreneur vom Kirzner'schen Entrepreneur (vgl. Abschnitt 5.4.2) wesentlich. Der Kirzner'sche Entrepreneur erkennt bestehende Preisdifferenzen auf den Märkten und nutzt diese zu seinem Vorteil, bis Imitatoren auftreten und die Preisdifferenzen verschwinden. Der Markt befindet sich zunächst im Ungleichgewicht und gelangt erst durch die Aktivitäten der Unternehmer ins Gleichgewicht. Dagegen wirkt der Schumpeter'sche Entrepreneur destabilisierend auf den sich im Gleichgewicht befindenden Markt und löst selbst das Ungleichgewicht aus, welches durch die

392 Schumpeter (1926), S. 125.

393 Vgl. Langlois (2002), S. 1 ff.; Brusoni et al. (2006), S. 13 f.; Meyer (1984), S. 148 f.

394 Vgl. Brusoni et al. (2006), S. 14.

395 Vgl. Baumol (2004), S. 1 ff.; Bianchi und Henrekson (2005), S. 366 f. 
Aktivitäten anderer Entrepreneure und Wirte wieder ins Gleichgewicht gebracht wird. ${ }^{396}$ Der Schumpeter'sche Entrepreneur greift aktiv ein, indem er die neuen Kombinationen in den Markt einführt. Er erschafft die EO selbst. ${ }^{397}$ Der Kirzner'sche Entrepreneur reagiert dagegen nur auf bereits existierende Veränderungen: „For me the function of the entrepreneur consists not of shifting the curves of cost or of revenues which face him, but of noticing that they have in fact shifted. "398

Der Schumpeter'sche Entrepreneur dient auch als Basis vieler neuerer Beiträge zur Entrepreneurship-Literatur. ${ }^{399}$ Beispielhaft sei das Modell von Holmes und Schmitz Jr. (1990) genannt. In diesem Modell bilden sich im Laufe der Zeit immer wieder Möglichkeiten zur Entwicklung neuer Produkte, die durch Ungleichgewichte, bedingt beispielsweise durch technischen Fortschritt und demographischen Wandel, entstehen. Nur in diesem Punkt unterscheidet sich das Modell vom ursprünglichen Schumpeter'schen Entrepreneur, der aus einem Gleichgewichtszustand heraus aktiv wird, wie oben erläutert wurde. Im Modell von Holmes und Schmitz Jr. besitzen Wirtschaftssubjekte unterschiedliche Fähigkeiten, diese Möglichkeiten zu nutzen und zu Produkten zu entwickeln - hierin liegt der Kern des Talents des Entrepreneurs und der Abgrenzung zwischen Schumpeter'schen Entrepreneuren und bloßen Wirten. Wenn ein Produkt in einer früheren Periode entwickelt und erfolgreich am Markt eingeführt wurde, dann muss das Produkt in den nächsten Perioden nur noch produziert werden, wofür Organisationsfähigkeiten benötigt werden, in denen sich alle Wirtschaftssubjekte laut Modell nicht unterscheiden. ${ }^{400}$

\subsubsection{Anwendung der Theorie auf Innovationsförderung}

Unternehmer, die sich für Fördermittel aus einem Innovationsförderprogramm bewerben, müssen Ideen für ein geplantes Innovationsprojekt vorweisen. Deswegen sind sie zum Zeitpunkt ihrer Bewerbung potentielle Schumpeter'sche Entrepreneure, während sie während der anschließenden Förderung notwendigerweise zu den innovativ tätigen Schumpeter'schen Entrepreneuren gehören. Die Frage, ob die geförderten Unternehmer vor ihrer Bewerbung eher den Schumpeter'schen Entrepreneuren oder den Wirten zuzurechnen waren, ist dagegen nicht eindeutig zu beantworten. Die Vermutung liegt nahe, dass eher diejenigen Unternehmer gefördert werden, die schon in der Vergangenheit Schumpeter'sche Entrepreneure waren. Aufgrund ihrer Innovationserfahrung ergreifen sie die EO Innovationsförderung möglicherweise eher als frühere Wirte, die we-

396 Vgl. Kirzner (1974), S. $72 \mathrm{ff}$.

397 Vgl. Grichnik (2006), S. 1308.

398 Kirzner (1974), S. 81.

399 Vgl. beispielsweise Henrekson (2007), S. 725 ff.; Baumol (1968), S. 65.

400 Vgl. Holmes und Schmitz Jr. (1990), S. 266 ff. 
der in Bezug auf Innovation noch in Bezug auf Innovationsförderung keine oder nur weit zurückliegende Erfahrungen aufweisen.

Die Unterscheidung zwischen den Unternehmertypen Entrepreneur und Wirt ist allerdings sehr schwierig, da sich ein Unternehmer im Laufe seines Unternehmerdaseins verändert. Ein und dieselbe Person kann während ihrer unternehmerischen Tätigkeit zeitweise Wirt und zeitweise Schumpeter'scher Entrepreneur sein. Trotzdem soll eine Differenzierung zwischen den beiden Typen vorgenommen werden. Es wird die Frage untersucht, ob tatsächlich eher diejenigen Unternehmer Fördermittel aus dem NIFP erhalten, die vor ihrer Bewerbung zu den Schumpeter'schen Entrepreneuren zählten. Als Indikator dafür, ob ein Unternehmer in der Vergangenheit eher zu den Schumpeter'schen Entrepreneuren oder zu den Wirten gehörte, bietet sich die Innovationstätigkeit des Unternehmens in der Vergangenheit an. Aufgrund des in Abschnitt 5.6.3 beschriebenen kumulativen Charakters der Innovationstätigkeit kann auch vom Umfang der aktuellen Innovationstätigkeit auf die Vergangenheit geschlossen werden.

Die Anwendung dieses Bereichs der Entrepreneurship-Theorie führt somit zurück zu Hypothese 5 in Abschnitt 4.5.1.1, nach der geförderte Unternehmer eine höhere Innovationstätigkeit aufweisen als nicht geförderte Unternehmer.

\subsection{Zusammenfassung der Ergebnisse und Hypothesen}

Im diesem Kapitel wurde geklärt, welche Wirtschaftssubjekte in Frage kommen, um einerseits generell als Unternehmer und andererseits als Entrepreneure im Sinne eines idealtypischen Unternehmers charakterisiert werden zu können. Es wurde herausgearbeitet, dass sich der Begriff des Unternehmers generell auf diejenigen Wirtschaftssubjekte bezieht, die die wesentlichen strategischen Entscheidungen des Unternehmens treffen. Um die Fragestellung zu beantworten, inwiefern sich Unternehmer, die Innovationsförderung erhalten bzw. sich für Innovationsfördermittel bewerben, von anderen Unternehmern unterscheiden, wurden Entrepreneurship-Theorieansätze verwendet, welche sich mit besonderen Fähigkeiten und Eigenschaften von Unternehmern beschäftigen. Es wurde untersucht, inwiefern sich theoretische Anhaltspunkte aus den EntrepreneurshipTheorien ergeben, die zur Erklärung beitragen können, warum nur ein Teil der Unternehmer innovativ tätig wird und warum nur ein Teil der innovativen Unternehmer Innovationsförderung beantragen.

Die eigentliche Tätigkeit eines Entrepreneurs erschließt sich in einer Synthese der verschiedenen in den vergangenen Abschnitten dargestellten Funktionen und Eigenschaften. ${ }^{401}$ Bianchi und Henrekson (2005) beschreiben Entrepreneurship als ,the ability and willingness of individuals, both on their own and

401 Vgl. Bonnet et al. (2008), S. 2 ff. 
within organizations to (i) innovate, i. e. perceive and create new economic opportunities, (ii) face uncertainty, i.e. introduce their ideas in the market, by making decisions on location, form and the use of resources and institutions; and (iii) manage their business by competing with others for a share of that market $^{\text {"402 }}$. In ihrer Definition erkennt man erstens den Schumpeter'schen Entrepreneur, zweitens den informierten Entrepreneur nach Casson und drittens das Element der Unsicherheit bzw. des Risikos, mit dem der Entrepreneur bei Knight konfrontiert wird.

Jeder Unternehmer übernimmt mit einer Unternehmensgründung Risiko, weil diese Wirtschaftstätigkeit mit Unsicherheit behaftet ist. Unter der Annahme, dass der Existenzgründer vor der Unternehmensgründung nicht arbeitslos war, entschließt er sich mit seinem Schritt in die Selbstständigkeit gegen eine sichere monatliche Entlohnung, die er als Angestellter erhalten würde, und für das Risiko des unsicheren Unternehmergewinns. Diese Risikoübernahme ist eine grundsätzliche Entscheidung. Das Ausmaß des übernommenen Risikos hängt von seiner konkreten Tätigkeit und den getroffenen strategischen Entscheidungen, insbesondere in Bezug auf die Innovationstätigkeit des Unternehmens, ab. Die Schumpeter'sche Innovationsfähigkeit hebt sich von den anderen Unternehmerfunktionen ab, weil sie sich nur auf bestimmte Phasen der Geschäftstätigkeit bezieht. Kein Unternehmer kann dauerhaft innovativ tätig sein. Für das zusätzlich übernommene Risiko durch die Innovationstätigkeit wird der Schumpeter'sche Entrepreneur mit einem risikobehafteten zusätzlichen Profit entschädigt.

Die Unternehmertätigkeit besteht zum großen Teil aus dem Treffen strategischen Entscheidungen sowie Organisation und Koordination der Produktion. Um Entscheidungen zu treffen, benötigt der Unternehmer Informationen. Wie alle anderen Wirtschaftssubjekte auch kann der Unternehmer seine Entscheidungen nicht auf der Grundlage vollständiger Information aufbauen. Unternehmer berücksichtigen also nur einen Bruchteil der prinzipiell verfügbaren Informationen bei ihrer Entscheidungsfindung und nutzen dabei jede Art von Informationsvorteilen, die sie besitzen. Dabei ist die Casson'sche Urteilsfähigkeit des Entrepreneurs auch für andere Entrepreneurship-Theorieansätze von Bedeutung. So braucht der Entrepreneur nach Cantillon eine ausgeprägte Urteilsfähigkeit, um zukünftige Preisbewegungen einzuschätzen, während der Knight'sche Entrepreneur seine Urteilsfähigkeit in Situationen struktureller Unsicherheit einsetzen muss. ${ }^{403}$ Während Cantillon und Knight aber die Einstellung zu Risiko und Unsicherheit als entscheidende Eigenschaft eines Entrepreneurs betonen, spielt diese bei Casson nur eine untergeordnete Rolle. Ebenso wie Kirzner und die anderen Vertreter der Österreichischen Schule betont Casson, wie wichtig es für

402 Bianchi und Henrekson (2005), S. 355.

403 Vgl. Casson (2002). 
den Entrepreneur ist, veränderte Rahmenbedingungen des Marktes wahrzunehmen und folgerichtige Entscheidungen zu treffen. Diese bewertenden Entscheidungen können auch die Initiierung von Schumpeter'schen Innovationen beinhalten. Der Schumpeter'sche Entrepreneur benötigt eine gut ausgeprägte Urteilsfähigkeit, um die mit Innovationen verbundenen Risiken und Chancen richtig einzuschätzen. ${ }^{404}$

In den vergangenen Abschnitten wurde das Konzept der EO verwendet, wobei sowohl Innovation als auch Innovationsförderung als mögliche EO betrachtet werden können. Verschieden ausgebildete Informationskompetenzen der Unternehmer stellen einen möglichen Grund dar, warum sich nur ein Teil der eigentlich für ein Innovationsförderprogramm in Frage kommenden Unternehmer tatsächlich um Fördermittel bewirbt. Zwei mögliche Erklärungen und Interpretationsmöglichkeiten konkurrieren miteinander. Erstens könnten diejenigen Unternehmer, die über eine besonders gut ausgeprägte Informationskompetenz verfügen, besonders gut über die Bereiche Innovation und Wirtschaftsförderung informiert sein. Schließlich fällt ihnen die Suche nach und die Verarbeitung von Informationen generell leichter als anderen Unternehmern, da sie eher dem Casson'schen Entrepreneur entsprechen.

Hypothese 6: Unternehmer, die sich um Innovationsförderung bewerben, sind in den Bereichen Innovation und Wirtschaftsförderung besser informiert als Unternehmer, die sich nicht für Förderprogramme bewerben.

Zweitens könnten sich Unternehmer wegen des beschriebenen Überangebots an Informationen in ihrer Informationssuche und -kompetenz auf bestimmte Bereiche beschränken. Manche Unternehmer spezialisieren sich demzufolge auf die Suche nach Informationen, die die Innovationstätigkeit betreffen. Andere Unternehmer spezialisieren sich dagegen auf die Suche nach für sie passenden Förderprogrammen und damit verbundene Bewerbungsformalitäten und entdecken deswegen häufiger passende Förderprogramme, für die sie sich anschließend bewerben.

Hypothese 7 (entgegen Hypothese 6): Unternehmer, die sich um Innovationsförderung bewerben, sind im Bereich Wirtschaftsförderung besser informiert als Unternehmer, die sich nicht für Förderprogramme bewerben. Aber sie sind nicht besser informiert, was den Bereich Innovation angeht.

404 Vgl. Casson (2002); Casson (1992), S. 189. 
Bei der Bewerbung um Fördermittel spielt neben der Informiertheit des Unternehmers außerdem die Fähigkeit Formulare auszufüllen eine wichtige Rolle. Denn um sich um ein Förderprogramm zu bewerben, muss der Unternehmer Formulare ausfüllen und einen Förderantrag stellen. Erhält er Fördermittel, so muss er eine ausführliche Dokumentation der Fördermittelverwendung vornehmen. Es ist anzunehmen, dass diese unternehmerische Fähigkeit mit der Informationskompetenz zusammenhängt. Wenn Unternehmer sich allerdings nicht für ein Programm bewerben, weil sie den damit einhergehenden bürokratischen Aufwand überschätzen, dann liegt eine Informationsasymmetrie vor.

\section{Hypothese 8: Der Bewerbungs- und Dokumentationsaufwand ist in der Realität nicht so groß wie in der Vorstellung derjenigen Unter- nehmer, die sich nicht für das NIFP beworben haben.}

Neben der Informiertheit der Unternehmer steht bei weiteren Ansätzen der Entrepreneurship-Literatur die Risikoeinstellung des Unternehmers im Fokus. Es gibt keinen Anhaltspunkt dafür, dass sich innovative und nicht innovative Unternehmer in ihrer Risikoeinstellung voneinander unterscheiden. Stattdessen informieren sich innovative Unternehmer über die mit der EO Innovation zusammenhängenden Risiken und Chancen und analysieren diese sorgfältig. Ihre Entscheidung für ein risikoreiches Innovationsprojekt treffen die Entrepreneure dann nach reiflicher Überlegung.

\section{Hypothese 9: Innovative und nicht innovative Unternehmer unter- scheiden sich nicht in ihrer Risikoeinstellung.}

Dagegen liefert die Risikoeinstellung des Unternehmers möglicherweise eine Erklärung für die Entscheidung, sich für ein Innovationsförderprogramm zu bewerben. Risikoscheue Unternehmer nehmen möglicherweise den Bewerbungsund Dokumentationsaufwand eher in Kauf. Denn der Erhalt der Fördermittel entschädigt sie durch die damit zusammenhängende Verringerung ihres eigenen finanziellen Risikos. Eher risikofreudige Unternehmer (die Knight'schen Entrepreneure) suchen sich dagegen andere Finanzierungsquellen für ihre Innovationsprojekte, die mit weniger organisatorischem und bürokratischem Aufwand verbunden sind.

Hypothese 10: Unternehmer, die sich um Innovationsförderung bewerben, sind risikoscheuer als Unternehmer, die sich nicht bewerben.

Abschließend wurde in diesem Kapitel diskutiert, inwiefern durch ein Innovationsförderprogramm geförderte Unternehmer vor ihrer Bewerbung eher den Schumpeter'schen Entrepreneuren oder den Wirten zuzurechnen waren. Zur Zeit 
der Förderung gehören die geförderten Unternehmer aufgrund ihrer Innovationstätigkeit zwangsläufig eher zu den innovativen Entrepreneuren im Schumpeter'schen Sinne. Bezogen auf die unmittelbare Vergangenheit ist zu vermuten, dass die geförderten Unternehmer ebenfalls zu den Schumpeter'schen Entrepreneuren gehörten. Aufgrund ihrer Erfahrung im Bereich F\&E bzw. Innovation ergreifen sie eine EO wie Innovationsförderung möglicherweise eher als frühere Wirte. Diese Argumentation deutet darauf hin, dass geförderte Unternehmer eine höhere Innovationstätigkeit aufweisen als nicht geförderte Unternehmer. Da diese Vermutung mit Hypothese 5 in Abschnitt 2.5.1.1 identisch ist, wird sie hier nicht noch einmal extra aufgeführt. Im folgenden Kapitel werden alle im Rahmen dieser Arbeit aufgestellten Hypothesen auf ihren empirischen Gehalt hin untersucht. 


\section{Empirische Untersuchung des Niedersächsischen Innovationsförderprogramms}

Im Mai/Juni 2007 wurde im Rahmen dieser Arbeit eine schriftliche Unternehmensbefragung durchgeführt, in der die Empfänger des NIFP sowie nicht geförderte Unternehmen aus den gleichen Wirtschaftszweigen zu den Themen Innovativität und Innovationsförderung befragt wurden. Im Folgenden werden die Ergebnisse dieser Befragung vorgestellt und die in den vorigen Kapiteln aufgestellten Hypothesen getestet.

Zunächst wird in Abschnitt 6.1 die Auswahl der befragten Unternehmen beschrieben. Die Verschickung der Fragebögen und der Rücklauf werden in Abschnitt 6.2 dargestellt, während in Abschnitt 6.3 der Aufbau des Fragebogens behandelt wird. In Abschnitt 6.4 folgt ein Vergleich der geförderten und nicht geförderten Unternehmen in Bezug auf Strukturdaten wie Unternehmensalter, Beschäftigtenzahl und Innovativität. In Abschnitt 6.5 werden die befragten Unternehmer hinsichtlich ihrer Risikoaversion und Informiertheit in den Bereichen Innovation und Wirtschaftsförderung verglichen. In Abschnitt 6.6 wird eine logistische Regression (Logit-Modell) vorgenommen, die den Zusammenhang zwischen der Förderung eines Unternehmens und der Ausprägung seiner Unternehmensstrukturdaten sowie der Eigenschaften und Fähigkeiten des jeweiligen Unternehmers untersucht. Das Kapitel schließt mit einer Zusammenfassung der Ergebnisse in Abschnitt 6.7.

\subsection{Auswahl der zu befragenden Unternehmen}

Im Rahmen der Unternehmensbefragung wurde der erstellte Fragebogen (vgl. Anhang C) an 205 Unternehmen verschickt, die im Zeitraum von 2004 bis Mai 2007 Fördermittel aus dem NIFP bewilligt bekommen haben. Dabei handelt es sich um eine Vollerhebung, d. h. alle geförderten Unternehmen aus diesem Zeitraum wurden angeschrieben. Unternehmen, die während des Zeitraums mehrfach Förderung aus dem Programm erhalten haben, wurden nur einmal angeschrieben.

Um eine Vergleichsgruppe von nicht geförderten Industrie- und Handwerksunternehmen zu befragen, wurden die Adressenlisten der bei den niedersächsischen Handwerkskammern sowie den Industrie- und Handelskammern eingetragenen Unternehmen genutzt. Die Vergleichsgruppe wurde so gewählt, dass nur Unternehmen aus denjenigen Wirtschaftszweigen angeschrieben wurden, die potentiell für das Innovationsförderprogramm in Frage kommen, so 
dass die Auswahl der zu befragenden Unternehmen bzgl. der Wirtschaftszweigzugehörigkeit keine Verzerrung beinhaltet.

Bezogen auf die zu befragenden Industrieunternehmen wurden nur die Wirtschaftszweige des Verarbeitenden Gewerbes befragt, weil der Tatbestand der förderfähigen Innovation im Sinne des NIFP auf diese Wirtschaftszweige begrenzt ist (vgl. Abschnitt 4.2). Es wurde bewusst keine weitere Eingrenzung der befragten Wirtschaftszweige innerhalb des Verarbeitenden Gewerbes vorgenommen, da Unternehmen aus jedem Wirtschaftszweig potentiell Innovationen im Sinne des Förderprogramms durchführen können. Die befragten Wirtschaftszweige sind in Anhang E dargestellt. Es wurden nur Industrieunternehmen mit Hauptsitz in Niedersachsen ausgewählt, die im Handelsregister eingetragen sind. ${ }^{405}$ Die zu befragenden Unternehmen wurden aus den Adressenlisten der ausgewählten Industriezweige geordnet nach Kammerbezirken mit Hilfe von Zufallszahlen bestimmt. Die Anzahl der befragten Unternehmen in einem Kammerbezirk entspricht dem Anteil des Kammerbezirks an der Gesamtzahl der Unternehmen in ganz Niedersachsen bezogen auf die ausgewählten Industriezweige. Im Rahmen dieser regional geschichteten Zufallsstichprobe wurden 1000 Industrieunternehmen angeschrieben.

Die Auswahl der Gewerke für die Vergleichsgruppe der zu befragenden Handwerksunternehmen orientiert sich an den in der Vergangenheit hauptsächlich geförderten Gewerken ergänzt um potentiell stark innovative Gewerke. Bei der Auswahl der potentiell innovativen Gewerke wurden die Ergebnisse von Lahner (2004) berücksichtigt, der Innovationsprozesse im Handwerk am Beispiel Niedersachsen untersucht. Dort findet sich eine Aufstellung der geförderten Gewerke des Förderprogramms „Innovationsförderung für das Niedersächsische Handwerk" im Zeitraum vom 01.07.1998 bis 30.06.2003, des Vorläuferprogramms des derzeitigen NIFP/Handwerk. ${ }^{406}$ Des Weiteren kommt die Untersuchung von Astor et al. (2006) zu dem Ergebnis, dass Elektrotechnik, Metallbau, Feinmechanik und Holz- und Tischlergewerbe als potentiell stark innovative Gewerke einzustufen sind. ${ }^{407}$ Die in dieser Arbeit befragten Gewerke sind in Anhang F dargestellt. Die zu befragenden Handwerksunternehmen wurden ana$\log$ zur Vorgehensweise bei den Industrieunternehmen aus den Adressenlisten der Gewerke, geordnet nach Kammerbezirken, mit Hilfe von Zufallszahlen bestimmt. Nach Aussortierung von Doppelungen wurden im Rahmen der beschriebenen Zufallsstichprobe insgesamt 983 Handwerksbetriebe angeschrieben.

Die angeschriebene Vergleichsgruppe bestand somit insgesamt aus 1983 angeschriebenen Industrie- und Handwerksunternehmen. Die Unternehmen der Vergleichgruppe und die Unternehmen des NIFP erhielten weitgehend identi-

405 Somit ist gewährleistet, dass keine Zweigniederlassungen angeschrieben werden, die sich im Allgemeinen nicht um Fördermittel bewerben.

406 Vgl. Lahner (2004), S. 306.

407 Vgl. Astor et al. (2006), S. 42. 
sche Fragebögen (vgl. Anhang C und D), die sich nur im Teil IV bezüglich der Teilnahme am NIFP unterschieden.

\subsection{Verschickung des Fragebogens und Rücklauf}

Alle angeschriebenen Unternehmen erhielten neben dem Fragebogen und einem frankierten Rückumschlag ein per Hand unterschriebenes Anschreiben. Die Unternehmen der Vergleichsgruppe erhielten ein Anschreiben der Verfasserin (vgl. Anhang $\mathrm{G}$ und $\mathrm{H}$ ), während die Unternehmen des NIFP ein Anschreiben der NBank erhielten (vgl. Anhang I). Im Anschreiben wurde explizit darauf hingewiesen, dass die Unternehmensbefragung eine unabhängige Untersuchung der Professur für Wirtschaftspolitik und Mittelstandsforschung der Georg-AugustUniversität Göttingen darstellt und nicht im Auftrag von Institutionen erfolgt, die am NIFP beteiligt sind. Den befragten Unternehmen wurde die Möglichkeit gegeben, sich telefonisch, postalisch oder per E-Mail an die Verfasserin zu wenden und Rückfragen zur Untersuchung zu stellen sowie sich im Internet auf einer extra eingerichteten Informationsseite über das Forschungsvorhaben zu informieren. Als Forschungsthemen, die die Befragung untersuchen sollte, wurden Innovationshemmnisse angegeben sowie Gründe, die Unternehmen davon abhalten, sich für Innovationsförderprogramme zu bewerben.

Der Rücklauf der durch das NIFP geförderten Unternehmen, die durch die NBank angeschrieben wurden, beläuft sich auf 114 ausgefüllte Fragebögen. Die Rücklaufquote beträgt 55,3\%. Diese Rücklaufquote ist vergleichsweise hoch und erklärt sich dadurch, dass die NBank den Fragebogen verschickt hat. Es ist also zu vermuten, dass die geförderten Unternehmen zum einen so zahlreich geantwortet haben, weil sie die NBank kennen und die Befragung nicht von einer unbekannten Institution durchgeführt wurde. Zum anderen besteht die Möglichkeit, dass die Unternehmen trotz der anonym ablaufenden Befragung glauben, dass sich eine Beteiligung an der Befragung positiv auf ihre eventuelle zukünftige Zusammenarbeit mit der die Fördermittel verwaltenden NBank auswirken könnte.

Von den 1983 Unternehmen der Vergleichsgruppe gaben 142 eine Rückmeldung (7,16\%). Darunter fanden sich 128 ausgefüllte Fragebögen $(6,45 \%)$. Diese Rücklaufquote ist sehr viel geringer als die Rücklaufquote für die geförderten Unternehmen. Trotzdem liegt sie mit 128 ausgefüllten Fragebögen hoch genug, um statistische Verfahren anwenden zu können. Es ist anzunehmen, dass ein Selektionseffekt vorliegt und dass nur Unternehmer, die sich für den Bereich Innovation interessieren, den Fragebogen ausgefüllt und zurückgesendet haben. $\mathrm{Da}$ es in dieser Untersuchung um Innovation und damit zusammenhängend um Innovationsförderung geht, hat dieser Selektionseffekt eher positive als negative Auswirkungen auf die Ergebnisse der Untersuchung. Denn diese Untersuchung 
hat nicht zum Ziel, ein repräsentatives Abbild der niedersächsischen Wirtschaftsstruktur zu liefern. ${ }^{408}$ Doch für einen Vergleich zwischen durch das NIFP geförderten Unternehmen und nicht geförderten Unternehmen sind die Befragungsergebnisse dagegen sehr hilfreich.

\subsection{Aufbau des Fragebogens}

Der von der Verfasserin erstellte Fragebogen für die geförderten Unternehmen (vgl. Anhang C) und der Fragebogen für die nicht geförderten Unternehmen (vgl. Anhang D) sind folgendermaßen aufgebaut: In Teil I wurden allgemeine Strukturdaten des Unternehmens sowie spezielle Daten aus dem Bereich F\&E/Innovation abgefragt. Teil II bestand aus Fragen, die die Informationsverarbeitungsfähigkeiten des Unternehmers betreffen. In Teil III sollten vorgegebene Persönlichkeitseigenschaften dahingehend eingeschätzt werden, ob sie wichtig für einen erfolgreichen Unternehmer seien. Außerdem wurde eine Aufgabe zur Bestimmung der Risikoaversion der Probanden gestellt. Teil IV des Fragebogens bestand aus Fragen zur Evaluation des NIFP. Die geförderten Unternehmen wurden zu Förderablauf, dem damit für sie verbundenen Zeitaufwand und zu ihrer Zufriedenheit mit dem Programmablauf befragt. Die nicht geförderten Unternehmen erhielten analoge Fragen, in denen sie den ihnen unbekannten Förderablauf bewerten und den damit verbundenen Aufwand für die geförderten Unternehmen einschätzen mussten. Für beide Gruppen identisch waren allgemeine Fragen zu Wirtschaftsförderprogrammen.

Um die Verständlichkeit der Frage- und Antwortmöglichkeiten für die befragten Unternehmer zu gewährleisten, wurde der Fragebogen detailliert und ausgiebig mit Vertretern des Wirtschaftsministeriums, der NBank und den Innovationsberatern der niedersächsischen Handwerkskammern diskutiert. Des Weiteren wurde ein Pretest mit zwei Unternehmern aus der Region Südniedersachsen durchgeführt, die in den letzten drei Jahren Fördermittel aus dem NIFP erhalten haben.

Um neben den Eigenschaften des Unternehmens auch die für diese Arbeit zentralen Eigenschaften des Unternehmers zu untersuchen, war es unbedingt erforderlich, dass der Unternehmer den Fragebogen selbst ausfüllt. Um dies zu gewährleisten, wurde zum einen im Anschreiben darauf hingewiesen, dass sich der Fragebogen direkt an den Geschäftsführer richtet. Zum anderen wurde im Fragebogen nach der Funktion des Ausfüllenden gefragt. In der überwiegenden Mehrzahl der Fälle (85,19\%) wurde der Fragebogen tatsächlich vom Geschäftsführer bzw. Unternehmenseigentümer ausgefüllt. 8,23 \% der Fragebögen wur-

408 Für eine repräsentative Darstellung der niedersächsischen Wirtschaft in Bezug auf F\&E und Innovation vgl. Niedersächsisches Institut für Wirtschaftsforschung (2008). 
den von leitenden Angestellten ausgefüllt. ${ }^{409} \mathrm{Da}$ diese als leitende Entscheidungsträger des Unternehmens auch unter die in dieser Arbeit verwendeten Unternehmerdefinition zählen (vgl. Abschnitt 5.2), wurden insgesamt 93,42\% der ausgefüllten Fragebögen von der Zielgruppe des Fragebogens ausgefüllt. Nur $2,88 \%$ der Fragebögen wurden von Angestellten in niedrigeren Positionen ausgefüllt und bei 3,70\% der zurückgesandten Fragebögen findet sich keine Angabe zur ausfüllenden Person.

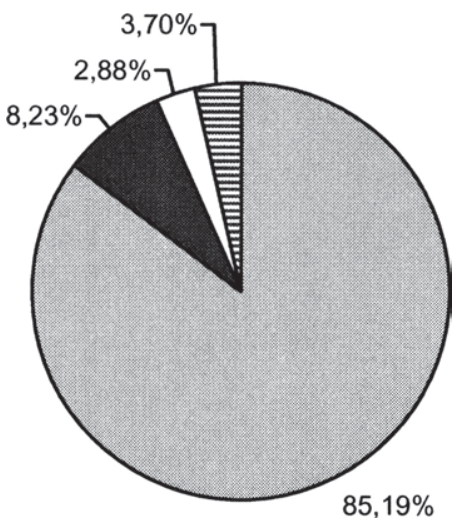

صeschäftsführer/Inhaber

Leitende Angestellte

口Angestellte

Ekeine Angabe

Abbildung 11: Funktion des Ausfüllenden im Unternehmen (Eigene Darstellung).

\subsection{Vergleich der geförderten und nicht geförderten Unterneh- men in Bezug auf Strukturdaten}

Insgesamt beträgt die Stichprobengröße dieser Untersuchung 242 Unternehmen. 120 dieser Unternehmen nahmen am NIFP teil. ${ }^{410}$ Ein Unternehmen bewarb sich für dieses Programm, ohne Fördermittel zu erhalten. Ein weiteres Unternehmen gab an, sich für das Programm beworben zu haben, ließ aber die Frage offen, ob die Bewerbung erfolgreich war. Deswegen wird dieses Unternehmen im Fol-

$409 \mathrm{Zu}$ den leitenden Angestellten zählen Abteilungsleiter (insbesondere der Bereiche Controlling, F\&E und Marketing) und Prokuristen.

$410 \mathrm{Zu}$ den 114 ausgefüllten Fragebögen aus der Verschickung der NBank, kommen noch sechs Fragebögen aus der Zufallsstichprobe der Kammern hinzu, die von durch das NIFP geförderten Unternehmen stammen. Diese sechs Unternehmen wurden daraufhin überprüft, ob sie mit einem der betrachteten geförderten Unternehmen identisch sind. Dieses war aber nicht der Fall, so dass sie problemlos in die weitere Untersuchung eingehen konnten. 
genden zu den Unternehmen gezählt, die sich für das NIFP beworben haben, deren Bewerbung aber nicht erfolgreich berücksichtigt wurde. 18 Unternehmen erhielten in den letzten drei Jahren keine Förderung aus dem NIFP, jedoch Fördermittel aus anderen Programmen. Acht Unternehmen bewarben sich in den letzten Jahren erfolglos um Fördermittel aus anderen Programmen. 94 Unternehmen bewarben sich nicht um Förderung und bekamen daher auch keine Fördermittel. Die letzten beiden Gruppen gaben mit großer Mehrheit nicht an, an welcher Art von Förderprogrammen sie teilgenommen haben bzw. für die sie sich beworben haben, so dass keine Informationen zur Einordnung dieser Förderprogramme vorliegen. Ein Überblick über die Verteilung befindet sich in Tabelle 1 .

Tabelle 1: Befragte Unternehmen nach Förderung

\begin{tabular}{ccccccc}
\hline NIFP- & $\begin{array}{c}\text { Nur } \\
\text { Förderung } \\
\text { erhalten } \\
\text { Bewerbung } \\
\text { um NIFP- } \\
\text { Förderung }\end{array}$ & $\begin{array}{c}\text { Andere } \\
\text { Förderung } \\
\text { erhalten }\end{array}$ & $\begin{array}{c}\text { Nur } \\
\text { Bewerbung } \\
\text { um andere } \\
\text { Förderung }\end{array}$ & $\begin{array}{c}\text { Keine } \\
\text { Bewerbung } \\
\text { und keine } \\
\text { Förderung }\end{array}$ & Gesamt \\
\hline Gesamt & 120 & 2 & 18 & 8 & 94 & 242 \\
\hline
\end{tabular}

Quelle: Eigene Auswertung.

Die Verteilung der befragten Unternehmen nach Kammerzugehörigkeit zeigt Tabelle 2.

Tabelle 2: Befragte Unternehmen nach Kammerzugehörigkeit

\begin{tabular}{lcccc}
\hline & $\begin{array}{c}\text { NIFP-Förderung } \\
\text { erhalten oder darum } \\
\text { beworben }\end{array}$ & $\begin{array}{c}\text { Andere Förderung } \\
\text { erhalten oder } \\
\text { darum beworben }\end{array}$ & $\begin{array}{c}\text { Keine Bewerbung } \\
\text { und keine } \\
\text { Förderung }\end{array}$ & Gesamt \\
\hline Handwerksbetrieb & 19 & 1 & 35 & 55 \\
Mischbetrieb $^{411}$ & 39 & 4 & 26 & 69 \\
Industriebetrieb & 58 & 21 & 33 & 112 \\
Keine Angabe & 6 & 0 & 0 & 6 \\
\hline Gesamt & 122 & 26 & 94 & 242 \\
\hline
\end{tabular}

Quelle: Eigene Auswertung.

411 Ein Mischbetrieb bezeichnet einen Betrieb, der sowohl Mitglied der Handwerkskammer als auch der Industrie- und Handelskammer ist. 
Bevor die unternehmerischen Strukturdaten im Einzelnen analysiert werden, gibt Tabelle 3 einen Überblick über die Verteilung der wichtigsten Strukturdaten der befragten Unternehmen.

Tabelle 3: Strukturdaten der befragten Unternehmen

\begin{tabular}{|c|c|c|c|c|c|}
\hline & Mittelwert & $\begin{array}{l}\text { Standard- } \\
\text { abweichung }\end{array}$ & $\begin{array}{c}\text { Minimal } \\
\text { angegebener } \\
\text { Wert }\end{array}$ & $\begin{array}{c}\text { Maximal } \\
\text { angegebener } \\
\text { Wert }\end{array}$ & $\begin{array}{c}\text { Anzahl der } \\
\text { Unternehmen }\end{array}$ \\
\hline Unternehmensalter & 34,20 & 35,06 & 1 & 169 & 238 \\
\hline Beschäftigtenzahl & 71,13 & 238,16 & 0 & 2700 & 241 \\
\hline $\begin{array}{l}\text { Beschäftigtenzahl } \\
\text { im Bereich F\&E }\end{array}$ & 4,77 & 11,88 & 0 & 120 & 236 \\
\hline Innovationsintensität & $11,92 \%$ & 0,20 & $0 \%$ & $100 \%$ & 229 \\
\hline $\begin{array}{l}\text { Beschäftigtenanteil } \\
\text { im Bereich F\&E }\end{array}$ & $19,56 \%$ & 0,28 & $0 \%$ & $100 \%$ & 235 \\
\hline Patentanzahl & 12,18 & 130,62 & 0 & 2000 & 237 \\
\hline $\begin{array}{l}\text { Anteil der Marktneu- } \\
\text { heiten am Umsatz }\end{array}$ & $21,93 \%$ & 0,26 & $0 \%$ & $100 \%$ & 225 \\
\hline
\end{tabular}

Quelle: Eigene Auswertung.

\subsubsection{Unternehmensalter}

Unternehmen, die Förderung aus dem NIFP erhalten bzw. sich darum beworben haben, weisen ein deutlich geringeres Unternehmensalter auf als die anderen betrachteten Unternehmensgruppen, wie am Boxplot in Abbildung 12 deutlich wird. So liegt der Median des Unternehmensalters der NIFP-Unternehmen (gefördert oder beworben) mit 17 Jahren deutlich unter 30,5 Jahren bei den Unternehmen, die sich nicht um Förderung beworben haben, und 29 Jahren bei den Unternehmen, die Fördermittel aus anderen Programmen erhalten bzw. sich darum beworben haben.

Die Variable Unternehmensalter kann nicht normalverteilt sein, da sie per Definition nur positive Werte annehmen kann. Außerdem treten wesentlich häufiger niedrige Werte auf als hohe, da die meisten Unternehmen nur eine begrenzte Lebensdauer haben. Da also die für parametrische Testverfahren erforderliche Voraussetzung der Normalverteilung nicht erfüllt ist, wird zur Überprüfung der Signifikanz der Gruppenunterschiede das nichtparametrische Testverfahren des Kruskal-Wallis-Tests verwendet. Der Kruskal-Wallis-Test bestätigt die Signifikanz der Gruppenunterschiede bei einem Signifikanzniveau von $1 \%$. Die Prüfgröße liegt bei 17,52 (zwei Freiheitsgrade). Der p-Wert liegt bei 0,0002 , d. h. mit einer Irrtumswahrscheinlichkeit von $0,02 \%$ kann die Nullhypothese, dass sich die Unternehmensgruppen hinsichtlich ihres Alters nicht 
unterscheiden, verworfen werden. Die Unternehmen des NIFP sind also signifikant jünger als die Unternehmen der Vergleichsgruppe.

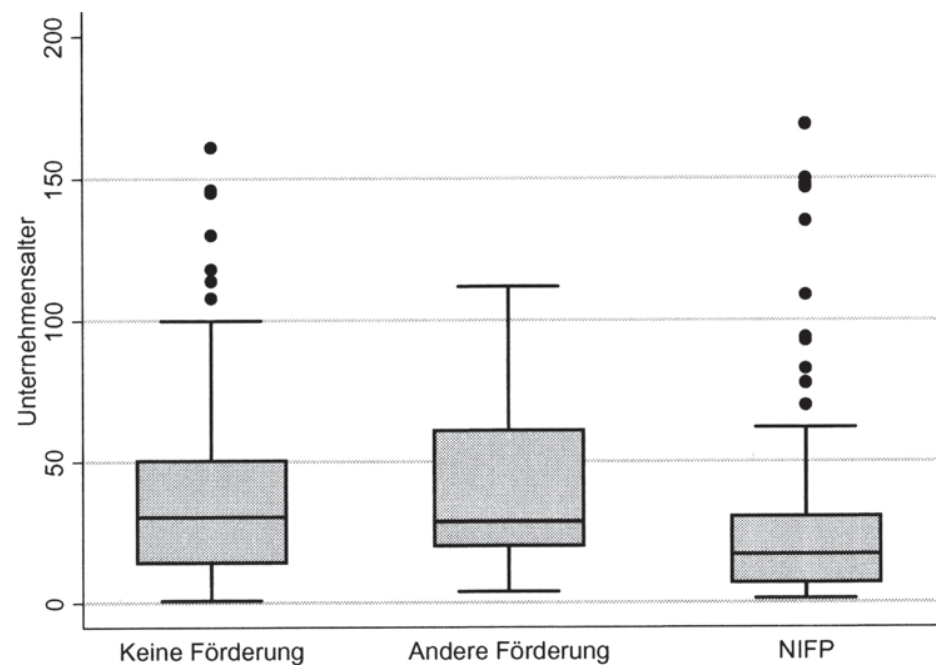

Abbildung 12: Boxplot des Unternehmensalters aufgeteilt nach Förderung (Eigene Darstellung).

\subsubsection{Unternehmensgröße}

Beim Thema Wirtschaftsförderung wird immer wieder vermutet, dass hauptsächlich große Unternehmen von Förderprogrammen profitierten. Die Untersuchung von Rammer und Binz (2006) bestätigt beispielsweise, dass im Zeitraum 2001-2003 innovative Großunternehmen in Deutschland deutlich häufiger Innovationsfördermittel erhielten als innovative KMU. ${ }^{412}$

Auch im Rahmen dieser Befragung haben einige nicht geförderte Unternehmen das Problem der Bevorzugung von Großunternehmen in Förderprogrammen angemerkt. So gab z. B. der Geschäftsführer eines Betriebes mit sechs Mitarbeitern aus dem Bereich Lkw-Zubehör folgenden Kommentar ab: „In der Regel werden Fördertöpfe von den Großunternehmen abgeschöpft. Ich halte das für Wettbewerbsverzerrung zu Lasten der kleinen und mittleren Unternehmen. " Ähnlich auch der Kommentar des Betriebsleiters einer Kfz-Reparaturwerkstatt mit drei Beschäftigten: „Leider fallen kleine Betriebe bis 5 Beschäftigte und einem Jahresumsatz unter 500.000 Euro meiner Meinung nach durch das Raster. “

412 Vgl. Rammer und Binz (2006), S. 139 ff. 
Für die durchgeführte Befragung weist der Vergleich der Unternehmensgrößen in Abbildung 13 und Abbildung 14 darauf hin, dass Unternehmen, die durch Wirtschaftsförderprogramme gefördert wurden oder sich für diese Programme beworben haben, im Schnitt deutlich mehr Beschäftigte aufweisen als Unternehmen, die nicht gefördert wurden bzw. sich nicht beworben haben. ${ }^{413}$ So liegt der Median der Unternehmen dieser zweiten Gruppe bei nur 10 Beschäftigten, während er für NIFP-Unternehmen (beworben und gefördert) bei $22 \mathrm{Be}$ schäftigten und für Unternehmen anderer Wirtschaftsförderprogramme (beworben und gefördert) bei 23,5 Beschäftigten liegt.

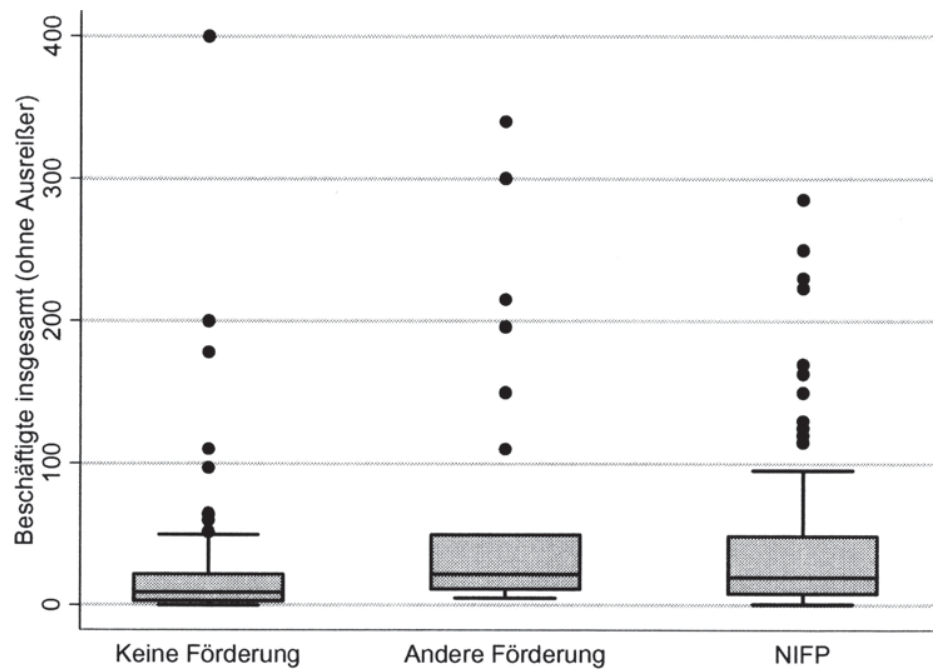

Abbildung 13: Boxplot der Beschäftigtenverteilung nach Förderung ${ }^{414}$ (Eigene Darstellung).

Von den Unternehmen, die sich nicht um Förderung beworben haben, sind auBerdem 49,46\% Kleinstunternehmen mit bis zu neun Beschäftigten. Diese Gruppe macht bei den NIFP-Unternehmen nur 29,51 \% bzw. bei den Unternehmen anderer Förderprogramme sogar nur 11,54 \% aus.

413 Die große Standardabweichung der Unternehmen, die Fördermittel aus anderen Programmen erhalten haben, erklärt sich aus der geringen Fallzahl von 26 Unternehmen. Diese Gruppe variiert stark in ihrer Beschäftigtenzahl.

414 Die Höchstanzahl der Beschäftigten wurde in der Graphik auf 400 begrenzt, so dass sieben Beobachtungen ausgeschlossen wurden (mit jeweils 520, 540, 680, 750, 1400, 1700 bzw. 2700 Beschäftigten), um die Aussagekraft der Graphik zu gewährleisten. 


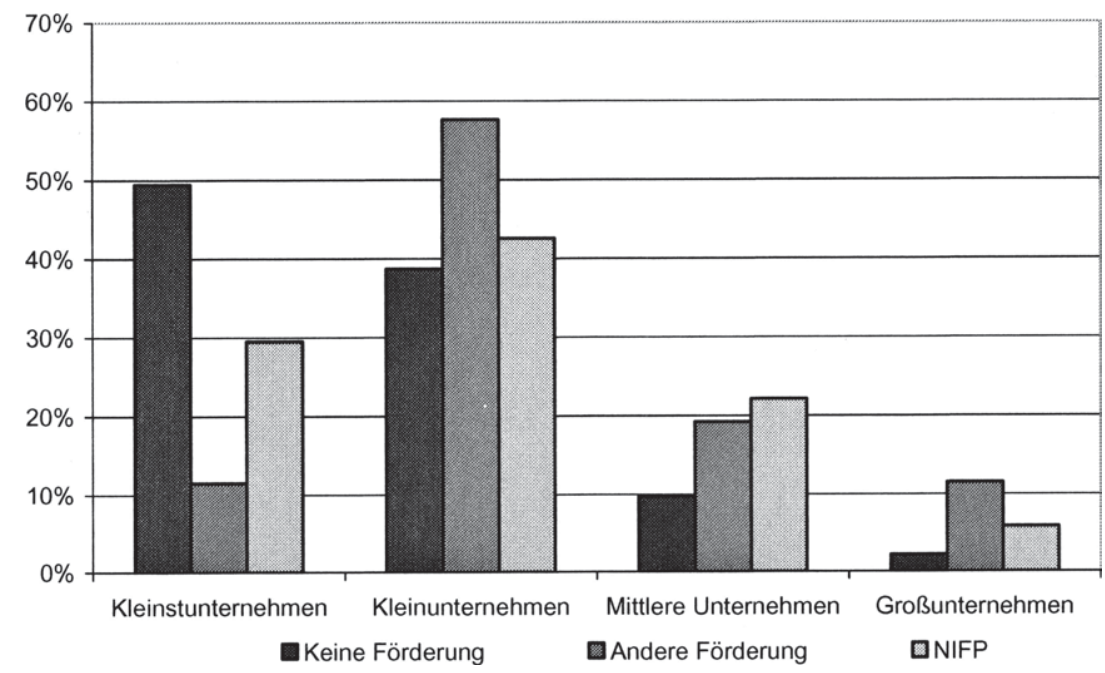

Abbildung 14: Säulendiagramm der Beschäftigtenverteilung nach Förderung ${ }^{415}$ (Eigene Darstellung).

Ebenso wie das Unternehmensalter ist auch die Beschäftigtenzahl eine Variable, die nur positive Werte annehmen kann und in der Regel nicht normalverteilt ist. Deswegen wird auch hier der Kruskal-Wallis-Test verwendet, welcher die Signifikanz der Gruppenunterschiede bei einem Signifikanzniveau von $1 \%$ bestätigt. Die Prüfgröße (zwei Freiheitsgrade) liegt bei 23,22 für alle Unternehmen. Mit einer Irrtumswahrscheinlichkeit von weniger als $1 \%$ kann die Nullhypothese, dass sich die Unternehmensgruppen hinsichtlich ihrer Beschäftigtenzahl nicht unterscheiden, verworfen werden. Werden die erwähnten Ausreißer aus der Analyse herausgenommen, bestätigt der Kruskal-Wallis-Test die Signifikanz der Gruppenunterschiede ebenfalls bei einem Signifikanzniveau von $1 \%$. Der FWert liegt dann bei 21,47 .

Jedoch darf dieses Testergebnis nicht isoliert betrachtet werden, da es neben der Unternehmensgröße noch weitere Einflussfaktoren gibt, die den Erhalt von Förderung beeinflussen könnten. So könnten kleine Unternehmen beispielsweise weniger innovativ sein und deswegen seltener Förderung erhalten als große. Aus diesem Grund wird der Zusammenhang zwischen Unternehmensgröße und dem Erhalt von Innovationsförderung in Abschnitt 6.6 in Kom-

415 Kleinstunternehmen haben bis zu neun, Kleinunternehmen zwischen 10 und 49, mittlere Unternehmen zwischen 50 und 249 und Großunternehmen mehr als 250 Beschäftigte, vgl. Brockmann (2005), S. 40. 
bination mit den anderen erhobenen Variablen abschließend in einer multivariaten Analyse überprüft.

\subsubsection{Innovativität}

Die in Abschnitt 4.5.1.1 aufgestellte Hypothese 5 (Geförderte Unternehmen weisen eine höhere Innovationstätigkeit auf als nicht geförderte Unternehmen) wird im Rahmen einer univariaten Analyse empirisch überprüft. Diese Hypothese wurde mit der Auswahl der zu fördernden Projekte durch die Bürokraten begründet, die sich an der unternehmerischen Innovationstätigkeit orientieren könnte. Deswegen werden im Unterschied zu den vorherigen Abschnitten die beiden Unternehmen, die sich erfolglos für das NIFP beworben haben, aus dem Vergleich der Unternehmensgruppen ausgeschlossen, so dass nur die tatsächlich durch das NIFP geförderten Unternehmen mit den Unternehmen, die sich nicht um Förderung beworben haben, sowie mit den Unternehmen verglichen werden, die andere Förderung bekommen haben. Da nicht bekannt ist, um welche Förderprogramme es sich handelt und inwiefern die Innovationstätigkeit bei der Auswahl eine Rolle spielen könnte, werden die Unternehmen, die sich erfolglos um andere Förderung beworben haben, ebenfalls aus dem Vergleich der Unternehmensgruppen ausgeschlossen.

Die Beantwortung der grundlegenden Frage, ob ein Unternehmen innovativ ist oder nicht, erfolgt in Anlehnung an die Definition des Mannheimer Innovationspanels. Laut dieser Definition haben innovative Unternehmen im Beobachtungszeitraum entweder Produktinnovationen (,neue oder merklich verbesserte Produkte bzw. Dienstleistungen, die ein Unternehmen auf den Markt gebracht hat ${ }^{\text {“416) }}$ ) bzw. Prozessinnovationen (,neue oder merklich verbesserte Fertigungsund Verfahrenstechniken bzw. Verfahren zur Erbringung von Dienstleistungen, die im Unternehmen eingeführt wurden ${ }^{6417}$ ) durchgeführt oder Aufwendungen für Innovationsaktivitäten getätigt, die noch nicht erfolgreich abgeschlossen sind oder ganz abgebrochen wurden. ${ }^{418}$ Diese vier Kriterien werden in der Unternehmensbefragung jeweils für die letzten drei Jahre abgefragt. Ein Unternehmen wird als innovativ betrachtet, wenn es mindestens eines dieser vier Kriterien erfüllt. Ein Überblick über die befragten Unternehmen geordnet nach Innovativität findet sich in Tabelle 4.

416 Vgl. Aschhoff et al. (2007), S. 3.

417 Vgl. Aschhoff et al. (2007), S. 3.

418 Vgl. Aschhoff et al. (2007), S. 3. 
Tabelle 4: Befragte Unternehmen geordnet nach Innovativität

\begin{tabular}{lcccr}
\hline & $\begin{array}{c}\text { NIFP-Förderung } \\
\text { erhalten }\end{array}$ & $\begin{array}{c}\text { Andere Förderung } \\
\text { erhalten }\end{array}$ & $\begin{array}{c}\text { Keine Förderung } \\
\text { erhalten und nicht } \\
\text { darum beworben }\end{array}$ & Gesamt \\
\hline Innovativ & 118 & 18 & 50 & 186 \\
Nicht innovativ & 2 & 0 & 40 & 42 \\
\hline Gesamt & 120 & 18 & 90 & 228 \\
\hline
\end{tabular}

Quelle: Eigene Auswertung.

$81,58 \%$ der befragten Unternehmen sind innovativ (186 von 228 Unternehmen). Die Gruppe der innovativen Unternehmen teilt sich auf in 118 Unternehmen, die durch das NIFP gefördert wurden $(63,44 \%), 18$ Unternehmen, die Fördermittel durch andere Wirtschaftsförderprogramme erhalten oder sich dafür beworben haben $(9,68 \%)$ und 50 Unternehmen, die in den letzten drei Jahren keine Wirtschaftsfördermittel erhalten haben $(26,88 \%)$. Unter den 42 nicht innovativen Unternehmen befinden sich 40 nicht geförderte Unternehmen $(95,24 \%), 2$ Unternehmen, die am NIFP teilgenommen haben $(4,76 \%)$ und kein einziges Unternehmen, das Fördermittel aus einem anderen Wirtschaftsförderprogramm erhalten hat. ${ }^{419}$

Die bloße Kategorisierung der befragten Unternehmen dahingehend, ob sie innovativ sind oder nicht, gibt jedoch keinen Aufschluss über das Ausmaß der Innovativität eines Unternehmens. Dazu wird eine metrische Größe benötigt. Wie im Folgenden diskutiert wird, zählen zu den gebräuchlichen Innovativitätsindikatoren die Ausgaben für F\&E bzw. Innovation, der Beschäftigtenanteil im F\&E-Bereich, die Patentanzahl sowie der Anteil der Marktneuheiten am Umsatz. Alle diese Indikatoren besitzen den Vorteil der einfachen Messbarkeit. Die ersten beiden genannten Indikatoren messen den Innovationsinput, während die Patentanzahl einen Throughput-Indikator darstellt und der Anteil der Marktneuheiten am Umsatz den Innovationsoutput misst.

Welcher Indikator verwendet wird, hängt von der verfolgten Fragestellung ab. Denn Input und Output von F\&E bzw. Innovation stehen nicht in einem deterministischen Zusammenhang. Die Inputindikatoren messen alle F\&E- und Innovationsanstrengungen - unabhängig davon, ob die Anstrengungen zu neuartigen Inventionen führen, die noch kein anderes Unternehmen hervorgebracht hat, und unabhängig davon, ob die Anstrengungen überhaupt zu Inventionen

419 Es ist durchaus plausibel, dass sich unter den durch das NIFP geförderten Unternehmen auch zwei nicht innovative Unternehmen befinden. Die überwiegende Mehrzahl der Förderfälle bezieht sich zwar auf die unternehmensinterne Innovationstätigkeit, aber es werden im Rahmen des NIFP/Handwerk auch Kooperationsvorhaben der Unternehmen mit Forschungseinrichtungen, also externe Innovationstätigkeit, gefördert. 
führen. Inputindikatoren liefern somit keinen Anhaltspunkt für den Stand des technischen Wissens innerhalb der Volkswirtschaft. Outputindikatoren geben zwar eher Aufschluss über die Erhöhung des technischen Wissens, aber sie vernachlässigen die Gesamtheit der getätigten Innovationsanstrengungen und -ausgaben in der Volkswirtschaft, weil sie nur das erfolgreiche Ergebnis der F\&Ebzw. Innovationstätigkeit messen. ${ }^{420}$

Die Bereiche F\&E und Innovation sind nicht identisch. Eine Innovation basiert auf einer meist durch F\&E erzeugten Invention, die am Markt oder innerhalb einer Organisation durchgesetzt wird (vgl. Abschnitt 2.1). Deswegen gehen Innovationsaufwendungen über die reinen F\&E-Aufwendungen hinaus. So gehören laut Oslo Manual zu den Innovationsaufwendungen neben den reinen F\&E-Aufwendungen für unternehmensinterne Forschung, den innovationsbezogenen Aufwendungen für Maschinen, Anlagen und Software und den Aufwendungen für externes Wissen und Mitarbeiterschulung zusätzlich noch die Aufwendungen für Markteinführung, Produktgestaltung und andere Vorbereitungen für Produktion und Vertrieb. ${ }^{421}$ Anstelle des absoluten Wertes der Innovationsaufwendungen wird häufig die relative Größe des Anteils der Innovationsaufwendungen am Umsatz (Innovationsintensität) als Indikator verwendet, wie beispielsweise beim Mannheimer Innovationspanel. ${ }^{422}$ Mit diesem relativen Indikator lassen sich Unternehmen unterschiedlicher Größe besser miteinander vergleichen. Dennoch unterliegt der Indikator der F\&E- bzw. Innovationsausgaben der Kritik, dass KMU nicht angemessen berücksichtigt werden. Denn sie haben keine formal abgegrenzte F\&E-Abteilung und weisen ihre Ausgaben und Mitarbeiter im F\&E-Bereich nicht differenziert aus. ${ }^{423}$

Um den Innovationsoutput zu messen, wird häufig die Anzahl der durch den Betrieb angemeldeten Patente verwendet. Problematisch am Indikator der Patente ist die Tatsache, dass viele Innovationen gar nicht patentiert werden, während andere durch mehrere Patente patentiert werden. Diese Unterschiede hängen neben anderen Faktoren von der jeweiligen Unternehmensbranche ab. Auch variiert der ökonomische und technologische Wert zwischen verschiedenen Patenten sehr stark. ${ }^{424}$ Ein weiteres grundsätzliches Problem der Verwendung von Patenten als Indikator für Innovationen ist, dass Patente vorwiegend zum Schutz der Inventionen des Unternehmens verwendet werden - also bevor diese in den Markt eingeführt werden. Nur wenn der Eintritt in den Markt erfolgreich ist, wird die Invention aber zur Innovation. Lediglich in diesem Fall

420 Vgl. Kuznets (1962), S. 19 f.; Patel und Pavitt (1995), S. 22 ff.; Acs und Audretsch (2005), S. 8.

421 Vgl. OECD und Eurostat (2005), S. $90 \mathrm{ff}$.

422 Vgl. Aschhoff et al. (2007), S. 4 ff.; OECD und Eurostat (2005), S. 74.

423 Vgl. Patel und Pavitt (1995), S. 21 und 35 ff.; Acs und Audretsch (2005), S. 8 f.

424 Vgl. OECD und Eurostat (2005), S. 22; Kuznets (1962), S. 36 f.; Patel und Pavitt (1995), S. 22 f.; Griliches (1990), S. 1669 ff.; Acs und Audretsch (2005), S. 9 f. 
steht das Patent als Indikator also auch tatsächlich für eine Innovation. Deshalb sollten Patente genauer als Throughputindikatoren bezeichnet werden. ${ }^{425}$ Trotz dieser augenscheinlichen Nachteile werden Patente aus folgenden Gründen in dieser Arbeit als dritter Innovationsindikator verwendet. Zum einen werden sie in der Literatur besonders häufig als Indikator verwendet, ${ }^{426}$ so dass die Vergleichbarkeit der hier vorliegenden Ergebnisse mit anderen Studien gewährleistet ist. Zum anderen impliziert ein Patent, dass die zugrunde liegende Invention ausreichend getestet wurde und als viel versprechend bewertet wird. Denn ansonsten lohnen sich Zeit und Kosten nicht, die für das Unternehmen mit der Beantragung eines Patents verbunden sind. ${ }^{427}$

Um anders als bei den Indikatoren Innovationsintensität, Anteil der im Bereich F\&E-Beschäftigten und Patentanzahl den erfolgreichen Markteintritt der Innovation stärker zu berücksichtigen, wird in dieser Arbeit als vierter Innovationsindikator der Anteil des Umsatzes mit Marktneuheiten am Gesamtumsatz verwendet. Dieser Indikator beschreibt das Ausmaß des Unternehmensumsatzes mit innovativen Produkten (Marktneuheiten). ${ }^{428}$

Zusammenfassend ist festzustellen, dass es keinen idealen Innovationsindikator gibt. Deswegen werden in dieser Arbeit zwei Innovationsinputs (Innovationsintensität, Anteil der im Bereich F\&E/Innovation Beschäftigten an der Gesamtbeschäftigtenzahl), ein Innovationsthroughput (Anzahl der Patente) und ein Innovationsoutput (Anteil des Umsatzes mit Marktneuheiten am Gesamtumsatz) als Innovationsindikatoren verwendet. Wie oben ausgeführt, befinden sich in der Gruppe der nicht geförderten Unternehmen $44,44 \%$ nicht innovative Unternehmen (40 von 90 Unternehmen). Bei einem Vergleich der Ausprägung der Innovativitätsindikatoren von geförderten und nicht geförderten Unternehmen ist es sinnvoll, nur Unternehmen mit Innovationstätigkeit zu betrachten. Deswegen werden die nicht innovativen Unternehmen aus der Analyse herausgenommen und nur die Gruppe der generell innovativ tätigen Unternehmen nach Förderung differenziert. Wie in Abbildung $15 \mathrm{zu}$ erkennen ist, sind die durch das NIFPUnternehmen (gefördert) bezogen auf den Indikator der Innovationsintensität deutlich innovativer (Median: 9,5\%) als Unternehmen, die sich gar nicht für Förderprogramme beworben haben (Median: $4 \%$ ) sowie Unternehmen, die sich für andere Wirtschaftsförderprogramme beworben haben (Median: $4 \%$ ). Da es sich um nicht normalverteilte Variablen handelt, wird auch hier der KruskalWallis-Test zur Überprüfung der Signifikanz der Gruppenunterschiede verwendet. Er bestätigt die Signifikanz der Gruppenunterschiede bei einem Signifikanzniveau von $1 \%$. Die Prüfgröße liegt bei 22,99 (zwei Freiheitsgrade). Der pWert liegt bei 0,0001 .

425 Vgl. Grupp (1997), S. 143 f.

426 Vgl. Grupp (1997), S. 158.

427 Vgl. Kuznets (1962), S. 36.

428 Vgl. näher zu diesem Indikator Grupp (1997), S. 198 f. 


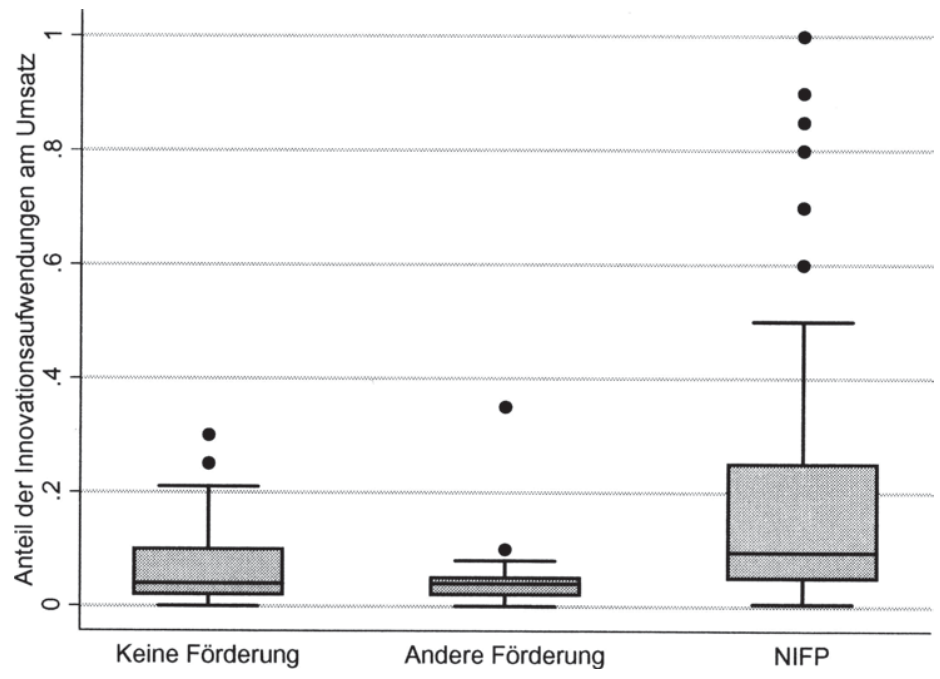

Abbildung 15: Boxplot der Innovationsintensität nach Förderung (Quelle: Eigene Darstellung).

Die anderen drei Innovativitätsindikatoren kommen zu ähnlichen Resultaten. So besitzen die Unternehmen, die Innovationsförderung erhalten haben, im Schnitt einen höheren Beschäftigtenanteil im Bereich F\&E bzw. Innovation (vgl. Abbildung 16), eine höhere Anzahl an Patenten (vgl. Abbildung 17) sowie einen höheren Anteil des Unternehmensumsatzes mit Marktneuheiten (vgl. Abbildung 18).

Schon die in den Boxplots dargestellten Verteilungen der vier Innovativitätsindikatoren machen deutlich, dass sich die drei nach Förderung differenzierten Gruppen in Bezug auf ihre Innovativität stark unterscheiden. Tabelle 5 stellt die Ergebnisse der durchgeführten Kruskal-Wallis-Tests dar, die die Signifikanz der Gruppenunterschiede für alle verwendeten Indikatoren auf dem $1 \% \mathrm{bzw}$. $5 \%$-Niveau bestätigen. 


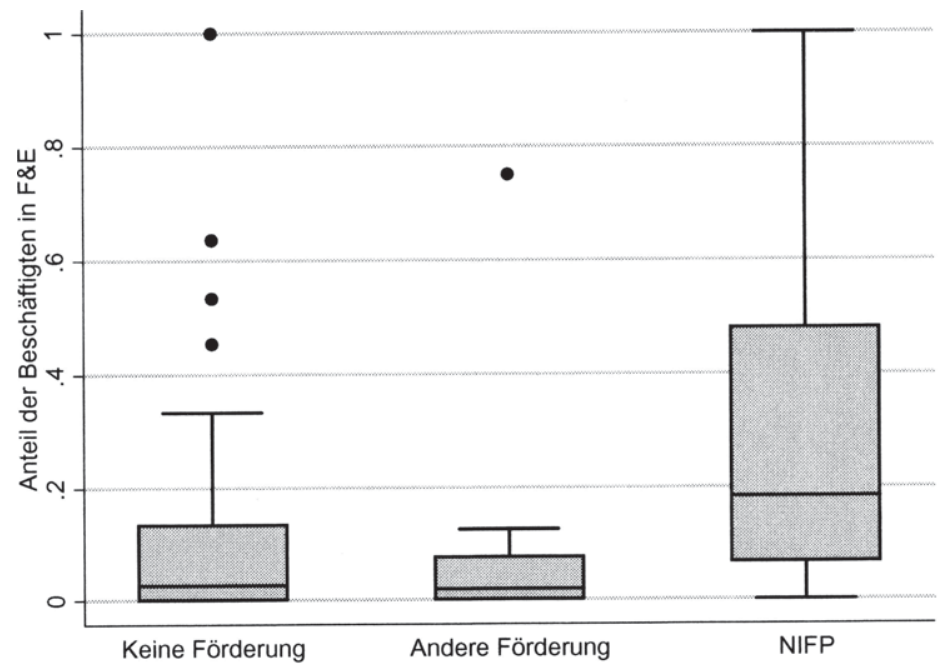

Abbildung 16: Boxplot des Beschäftigtenanteils im Bereich F\&E nach Förderung (Eigene Darstellung).

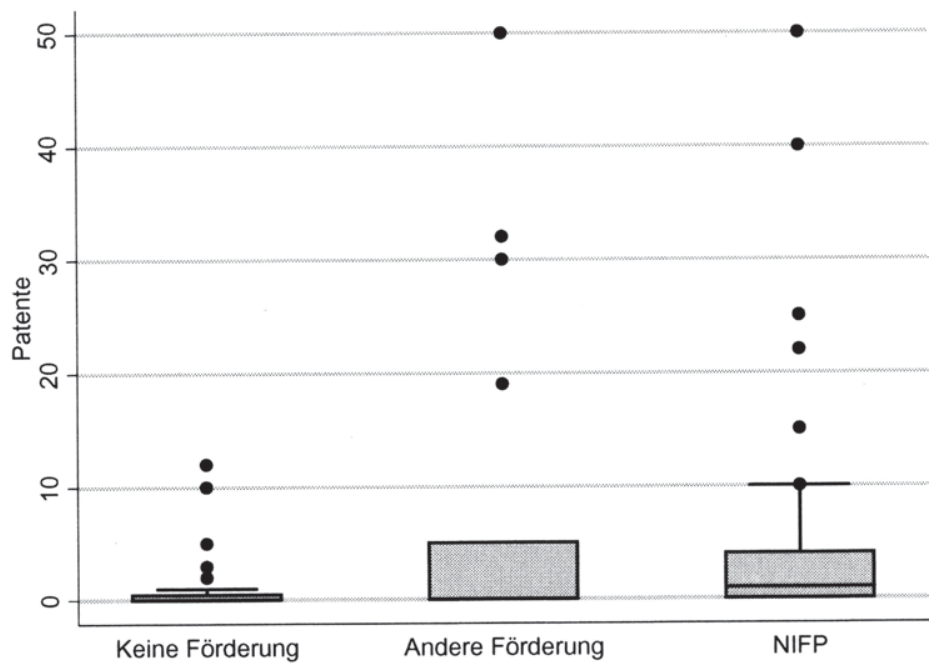

Abbildung 17: Boxplot der Patente ${ }^{429}$ nach Förderung (Eigene Darstellung).

429 Die Höchstanzahl der Patente wurde in der Graphik auf 50 begrenzt, so dass drei Beobachtungen von Unternehmen ausgeschlossen wurden (mit jeweils 130, 180 bzw. 2000 Patenten), um die Aussagekraft der Graphik zu gewährleisten. 


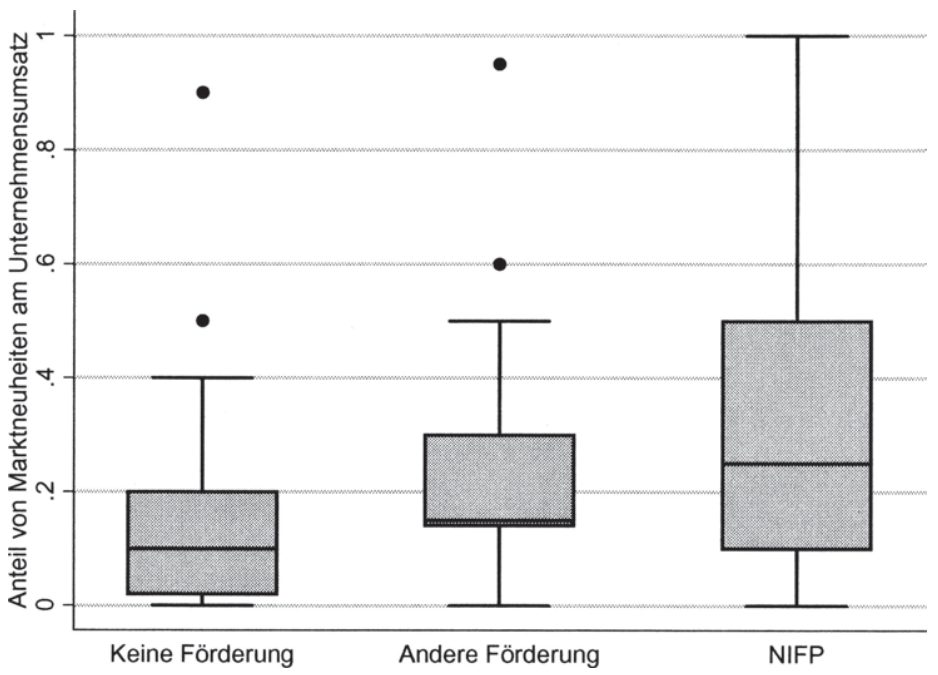

Abbildung 18: Boxplot des Anteils von Marktneuheiten am Unternehmensumsatz nach Förderung (Eigene Darstellung).

Tabelle 5: Signifikanz der Gruppenunterschiede bezogen auf Innovativitätsindikatoren

\begin{tabular}{|c|c|c|}
\hline Innovationsindikator & Prüfgröße (2 FG) & p-Wert \\
\hline Innovationsintensität & 22,99 & $0,0001 * * *$ \\
\hline Beschäftigtenanteil im Bereich F\&E & 38,15 & $0,0001 * * *$ \\
\hline Patente (ohne Ausreißer) ${ }^{430}$ & 8,53 & $0,0140 * *$ \\
\hline Anteil von Marktneuheiten am Umsatz & 17,46 & $0,0002 * * *$ \\
\hline
\end{tabular}

* $10 \%$,** $5 \%, * * * 1 \%$.

Quelle: Eigene Auswertung.

Der Besitz einer unternehmenseigenen Abteilung für Forschung und Entwicklung gibt ebenfalls Aufschluss über die Innovativität des Unternehmens. $14,29 \%$ der nicht geförderten Unternehmen, $41,03 \%$ der durch das NIFP geförderten Unternehmen sowie 33,33 \% der durch andere Förderprogramme geförderten Unternehmen besitzen eine eigene F\&E-Abteilung. Alle Zahlen beziehen sich wiederum nur auf die innovativ tätigen Unternehmen.

430 Vgl. Fn. 429. 


\subsubsection{Bewertung von Innovationshemmnissen}

Die befragten Unternehmen sollten sieben aufgeführte Innovationshemmnisse auf einer Skala von 1 (= sehr große Bedeutung) bis 5 (= keine Bedeutung) bewerten. Die Ergebnisse finden sich in Tabelle 6. Da innovativ tätigen Unternehmen bestehende Innovationshemmnisse stärker bewusst sein könnten und sie diese deswegen als wichtiger bewerten könnten als nicht innovative Unternehmen, ${ }^{431}$ bezieht sich die Auswertung wiederum nur auf innovativ tätige Unternehmen. Die Ergebnisse der Bewertung finden sich in Abbildung 19.

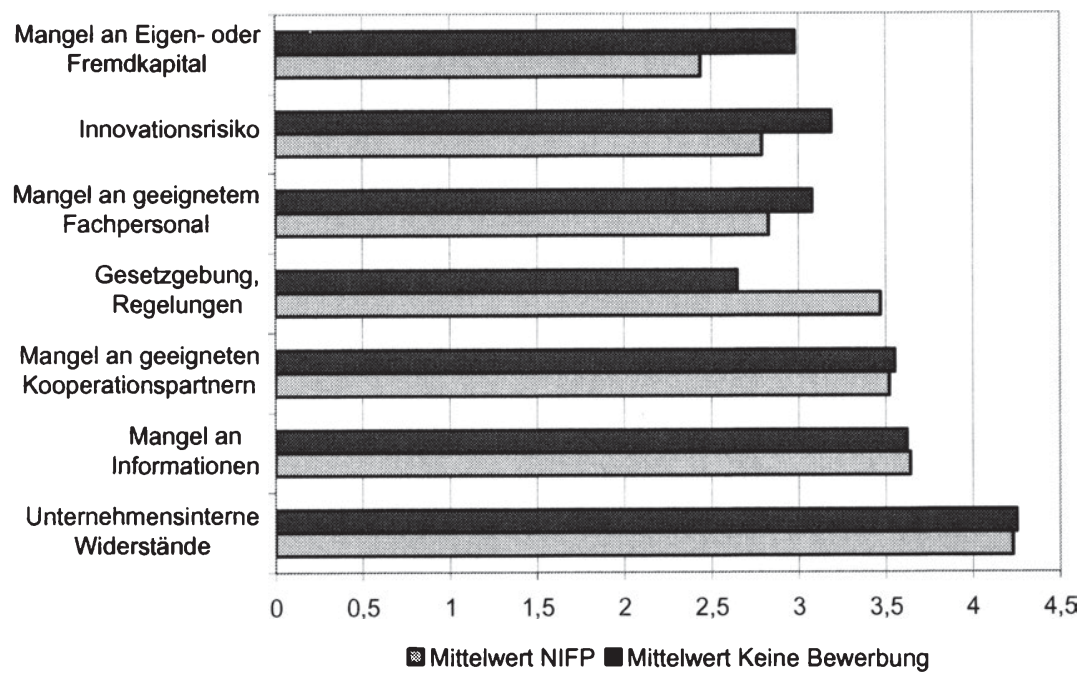

Abbildung 19: Innovationshemmnisse nach Förderung (Eigene Darstellung).

Unternehmen, die durch das NIFP gefördert wurden bzw. sich dafür beworben haben, bewerten das Innovationsrisiko und den Mangel an Eigen- und Fremdkapital als die wichtigsten Innovationshemmnisse. Diese Unternehmen messen den genannten Hemmnissen außerdem eine größere Wichtigkeit bei als Unternehmen, die keine Fördermittel erhalten und sich auch nicht um Förderung beworben haben. Der Unterschied in der Bewertung ist auf dem $5 \%$ bzw. $10 \%$ Niveau signifikant (bestätigt durch den Wilcoxon-Rangsummentest auf dem $5 \%$ - bzw. $10 \%$-Signifikanzniveau, vgl. Tabelle 6 ). ${ }^{432}$

431 Vgl. Mohnen et al. (2008), S. 7.

432 Da die Bewertung der Innovationshemmnisse auf einer Skala von 1 bis 5 eine ordinal skalierte Variable darstellt, wird der Wilcoxon-Rangsummentest verwendet, um zu untersuchen, ob die beiden Gruppen hinsichtlich ihrer Bewertung als signifikant verschie- 
Tabelle 6: Innovationshemmnisse

\begin{tabular}{|c|c|c|c|c|c|c|}
\hline $\begin{array}{l}\text { Innovations- } \\
\text { hemmnisse }\end{array}$ & $\begin{array}{c}\text { NIFP- } \\
\text { Förderung } \\
\text { erhalten } \\
\text { oder darum } \\
\text { beworben } \\
\text { (Mittelwert) }\end{array}$ & $\begin{array}{l}\text { Keine } \\
\text { Bewerbung } \\
\text { und keine } \\
\text { Förderung } \\
\text { (Mittelwert) }\end{array}$ & $\begin{array}{l}\text { NIFP- } \\
\text { Förderung } \\
\text { erhalten } \\
\text { oder darum } \\
\text { beworben } \\
\text { (Median) }\end{array}$ & $\begin{array}{l}\text { Keine } \\
\text { Bewerbung } \\
\text { und keine } \\
\text { Förderung } \\
\text { (Median) }\end{array}$ & $\begin{array}{c}\text { Mann- } \\
\text { Whitney- } \\
\text { U-Prüfgröße } \\
\text { Z-Wert }\end{array}$ & $\operatorname{Pr}$ \\
\hline Innovationsrisiko & 2,79 & 3,19 & 3 & 3 & 2,06 & $0,04 * *$ \\
\hline $\begin{array}{l}\text { Mangel an Eigen- } \\
\text { oder Fremdkapital }\end{array}$ & 2,44 & 2,98 & 2 & 3 & 2,45 & $0,01 * *$ \\
\hline $\begin{array}{l}\text { Mangel an geeig- } \\
\text { netem Fachperso- } \\
\text { nal }\end{array}$ & 2,83 & 3,08 & 3 & 3 & 1,32 & 0,19 \\
\hline $\begin{array}{l}\text { Unternehmensin- } \\
\text { terne Widerstände }\end{array}$ & 4,23 & 4,25 & 4 & 4,5 & 0,21 & 0,84 \\
\hline $\begin{array}{l}\text { Mangel an Infor- } \\
\text { mationen (Techno- } \\
\text { logie, Marketing } \\
\text { etc.) }\end{array}$ & 3,64 & 3,62 & 4 & 4 & $-0,05$ & 0,96 \\
\hline $\begin{array}{l}\text { Mangel an geeig- } \\
\text { neten Kooperati- } \\
\text { onspartnern }\end{array}$ & 3,52 & 3,55 & 4 & 3 & $-0,04$ & 0,97 \\
\hline $\begin{array}{l}\text { Gesetzgebung, } \\
\text { Regelungen }\end{array}$ & 3,47 & 2,65 & 3 & 3 & $-3,76$ & $0,00 * * *$ \\
\hline
\end{tabular}

Quelle: Eigene Auswertung.

Dass Unternehmer, die Mittel aus dem NIFP erhalten bzw. sich darum beworben haben, das Innovationsrisiko als ein wichtigeres Innovationshemmnis bewerten als Unternehmer, die sich nicht um Förderung beworben und dementsprechend auch keine Förderung erhalten haben, weist darauf hin, dass die erste Gruppe risikoscheuer ist. Diese Fragestellung wird in Abschnitt 6.5.2 ausführlicher untersucht. Dass die erste Gruppe den Mangel an Eigen- und Fremdkapital als ein wichtigeres Innovationshemmnis bewertet als die zweite, deutet darauf hin, dass die erste Gruppe Schwierigkeiten hat, Kapital für ihre Innovationsprojekte zu beschaffen, und sich gerade deswegen für ein Innovationsförderprogramm bewirbt. Des Weiteren ist der Mangel an geeignetem Fachpersonal sowie der Mangel an geeigneten Kooperationspartnern ein wichtigeres Innovationshemmnis für NIFP-Unternehmer (beworben und gefördert) als für Unternehmer, die sich nicht um Förderung beworben und keine Förderung erhalten haben. Aller-

den angesehen werden können. Er funktioniert ähnlich wie der Kruskal-Wallis-Test, vergleicht aber im Unterschied zu diesem nur zwei Gruppen miteinander. 
dings sind diese beiden Innovationshemmnisse auf dem $10 \%$-Niveau nicht signifikant.

Unternehmer, die sich nicht um Förderung beworben und keine Förderung erhalten haben, bewerten Gesetzgebung bzw. Regelungen als signifikant wichtigere Innovationshemmnisse als NIFP-Unternehmer. Unternehmensinterne Widerstände sowie der Mangel an Informationen werden von beiden Gruppen in ihrer Wichtigkeit ähnlich bewertet.

\subsubsection{Fazit}

Die univariaten Analysen ergeben, dass sich Unternehmen, die Förderung aus dem NIFP erhalten bzw. sich darum beworben haben, von Unternehmen, die sich nicht um Förderung beworben und dementsprechend keine Förderung erhalten haben, in Bezug auf Unternehmensalter, Unternehmensgröße (gemessen mit Hilfe der Beschäftigtenzahl) und Innovativitätsgrad unterscheiden. NIFPUnternehmen sind jünger und haben mehr Beschäftigte als Unternehmen, die sich nicht um Förderung beworben haben. Außerdem sind sie deutlich innovativer, unabhängig davon, ob die Innovationsintensität, der Beschäftigtenanteil im Bereich F\&E, die Anzahl der Patente oder der Anteil der Marktneuheiten am Umsatz als Innovativitätsindikator verwendet werden.

Hypothese 5 aus Abschnitt 4.5.1.1 wird also bestätigt: Geförderte Unternehmen sind tatsächlich innovativer als nicht geförderte Unternehmen. Auch dieses Ergebnis sollte jedoch nicht isoliert betrachtet werden, da es weitere mögliche Einflussfaktoren auf die Förderung gibt. Alle Einflussfaktoren werden im Rahmen der multivariaten Analyse in Abschnitt 6.6 gemeinsam untersucht. $\mathrm{Zu}-$ nächst folgen jedoch die univariaten Untersuchungen des Informationsverhaltens, der Risikoaversion und der Bewertung der Wichtigkeit verschiedener Unternehmereigenschaften durch den Unternehmer.

\subsection{Vergleich der geförderten und nicht geförderten Unter- nehmen in Bezug auf Unternehmereigenschaften}

Während sich die vorhergehenden Abschnitte mit dem Unterschied zwischen geförderten und nicht geförderten Unternehmen beschäftigten, liegt in diesem Abschnitt in Anknüpfung an die Erkenntnisse der Entrepreneurship-Theorie (vgl. Kapitel 5) der Fokus nun nicht mehr auf dem Unternehmen, sondern stattdessen auf dem Unternehmer. Es geht darum herauszufinden, ob bestimmte Eigenschaften und Fähigkeiten bei geförderten und nicht geförderten Unternehmern bzw. bei Unternehmern, die sich für Förderprogramme beworben haben, und Unternehmern, die sich nicht für Förderprogramme beworben haben, unterschiedlich ausgeprägt sind. Zunächst wird die Informiertheit des Unternehmers 
und daran anschließend seine Risikoeinstellung untersucht. Abschließend geht es um die Bewertung von vorgegebenen Unternehmereigenschaften durch die befragten Unternehmer selbst. Es werden im Folgenden nur die Fragebögen ausgewertet, die vom Unternehmer selbst ausgefüllt wurden (vgl. Abschnitt 6.3).

\subsubsection{Die Informiertheit des Unternehmers}

Eine wesentliche Fähigkeit des Unternehmers ist seine Informationskompetenz und Urteilsbildung in wirtschaftlichen Entscheidungsprozessen. Der Unternehmer ist ein Informationsmanager (vgl. Abschnitt 5.4.3). In diesem Abschnitt wird die Informiertheit von Unternehmern, die Fördermittel erhalten bzw. sich darum beworben haben, und Unternehmern, die sich nicht um Förderung beworben haben, verglichen, um die im Abschnitt 5.4.5 aufgestellten Hypothesen zu überprüfen. Hypothese 6 lautete: Unternehmer, die sich um Innovationsförderung bewerben, sind in den Bereichen Innovation und Wirtschaftsförderung besser informiert als Unternehmer, die sich nicht für Förderprogramme bewerben. Hypothese 7 dagegen lautete: Unternehmer, die sich um Innovationsförderung bewerben, sind im Bereich Wirtschaftsförderung besser informiert als Unternehmer, die sich nicht für Förderprogramme bewerben. Aber sie sind nicht besser informiert, was den Bereich Innovation angeht.

Die Messung der unternehmerischen Informiertheit mit Hilfe eines schriftlichen Fragebogens ist allerdings schwierig. In älteren Untersuchungen wird häufig die Nutzung von Informations- und Kommunikationstechnologien als Indikator für das unternehmensinterne Informationsmanagement gesehen. Im Einzelnen werden die Nutzung interner Netzwerke, die elektronische Abwicklung von Geschäftsvorfällen, das Anbieten eigener Produkte mit Hilfe von elektronischen Katalogen sowie die Internet- und die Multimedianutzung untersucht. ${ }^{433}$ Allerdings dürften die Unterschiede zwischen Unternehmen in Bezug auf diese Indikatoren heute nicht mehr so gravierend sein wie noch vor ein paar Jahren, da die Nutzung von Informations- und Kommunikationstechnologien mittlerweile für die meisten Unternehmen zur Selbstverständlichkeit geworden ist. Deswegen wird in dieser Arbeit ein neuer Ansatz entwickelt.

Generell benötigen Unternehmer Informationen aus verschiedenen Informationsquellen, um interessante Gewinnmöglichkeiten zu entdecken. ${ }^{434}$ Dies gilt auch für die Bereiche Innovation und Wirtschaftsförderung. Auf dieser Argumentation aufbauend wird die Schlussfolgerung gezogen, dass ein Unternehmer desto besser informiert ist, je mehr verschiedene Informationsquellen er in seiner Entscheidungsfindung berücksichtigt. In dieser Befragung wird die Bewertung der Wichtigkeit der angegebenen Informationsquellen abgefragt. Wenn

433 Vgl. z. B. Förderer et al. (1998), S. 25 ff., die diese Indikatoren in einer Unternehmensbefragung zum Thema Innovation und KMU verwenden.

434 Vgl. Casson (2003). 
ein Unternehmer eine Informationsquelle in der Realität tatsächlich stark nutzt, ist davon auszugehen, dass er sie im Rahmen einer Befragung als relativ wichtig bewertet. Unternehmer, die eine Vielzahl von Informationsquellen nutzen und infolgedessen als relativ wichtig bewerten, gelten als umfassend informiert. Unternehmer, die wenige Informationsquellen nutzen und dementsprechend nur wenige Quellen als wichtig bewerten, werden dagegen als schlechter informiert angesehen.

Die befragten Unternehmer sollten die Bedeutung einer Reihe von aufgeführten Informationsquellen für ihr Unternehmen auf einer Likert-Skala von 1 (sehr große Bedeutung) bis 5 (keine Bedeutung) angeben, um sich erstens über Wirtschaftsförderprogramme und zweitens über Innovation zu informieren. Die Ergebnisse der Befragung weisen auf einen deutlichen Unterschied in der Informiertheit zwischen geförderten und nicht geförderten Unternehmern hin.

Zunächst werden die Ergebnisse der Bewertung der einzelnen Informationsquellen durch Unternehmer, die Fördermittel erhalten bzw. sich darum beworben haben, und Unternehmern, die sich nicht für Förderprogramme beworben haben, einander gegenübergestellt. Es wird deutlich, dass NIFPUnternehmer (gefördert oder beworben) im Schnitt niedrigere Werte für alle Informationsquellen angeben als Unternehmer, die sich nicht um Förderung beworben haben. Die Mediane und Mittelwerte der beiden Gruppen weichen deutlich voneinander $a b, d$. $h$. Unternehmer der ersten Gruppe weisen den meisten angegebenen Informationsquellen eine stärkere Bedeutung zu als Unternehmer der zweiten Gruppe. Das bedeutet wiederum, dass Unternehmer der ersten Gruppe die Quellen annahmegemäß auch in einem höheren Ausmaß nutzen als Unternehmer der zweiten Gruppe. Dieser Sachverhalt wird auch in den folgenden beiden Abbildungen deutlich, die die Mittelwerte der Bewertung der Informationsquellen darstellen - in Abbildung 20 bezogen auf den Bereich Wirtschaftsförderung und in Abbildung 21 bezogen auf den Bereich Innovation.

Diese Darstellung gibt jedoch nur die Bewertung der einzelnen Informationsquellen durch die Unternehmer an. Um die Informiertheit der Unternehmer insgesamt zu vergleichen, wird im Folgenden ein Informiertheitsindikator gebildet, in den die Bewertungen aller Informationsquellen mit einfließen. Für jeden Unternehmer wird der Mittelwert seiner Bewertungen aller Informationsquellen gebildet. Da dieser Mittelwert steigt, je weniger wichtig die Informationsquellen für den Unternehmer sind und demzufolge also auch je weniger der Unternehmer diese Informationsquellen praktisch nutzt, bildet diese metrische Variable gewissermaßen die Uninformiertheit des Unternehmers ab. 

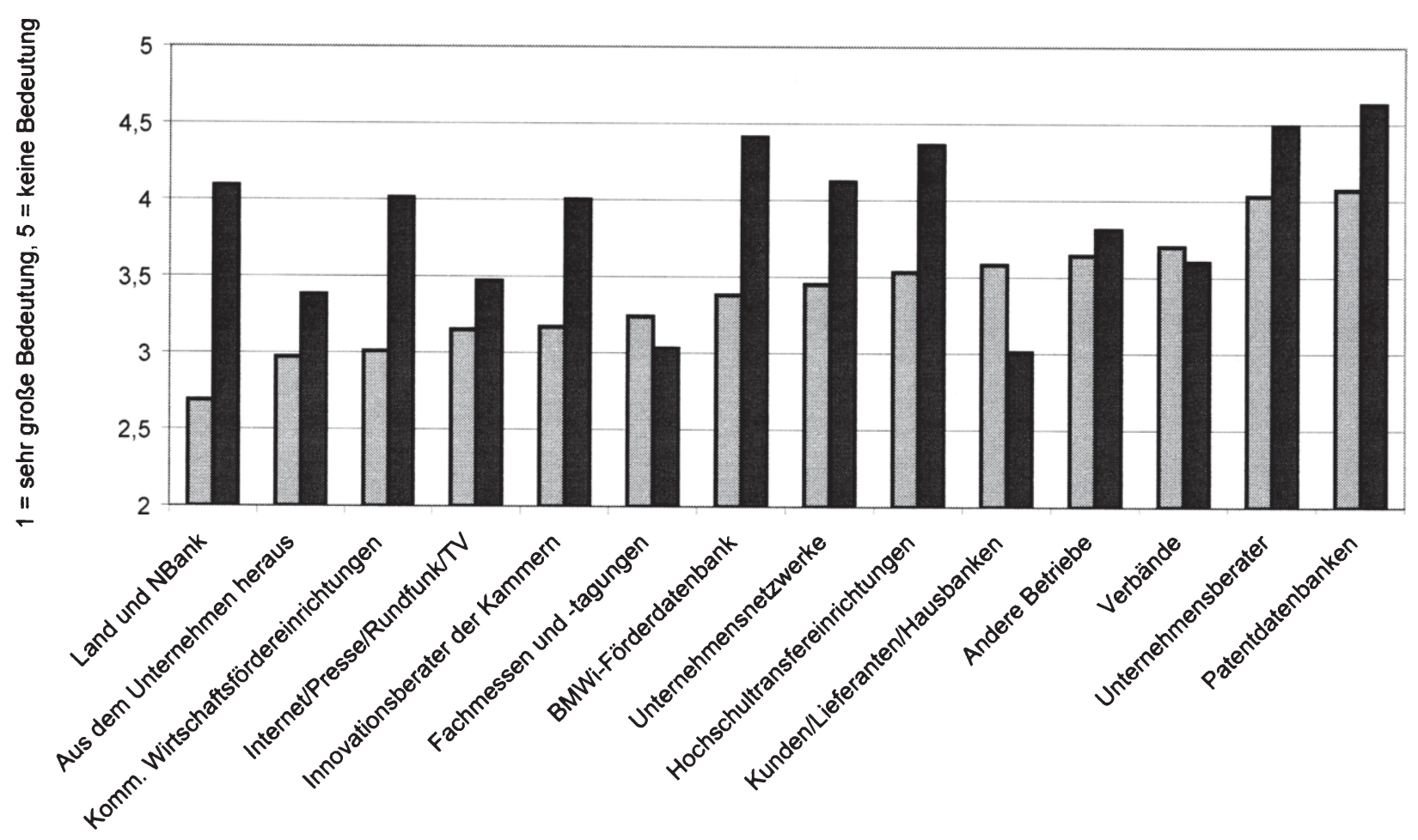

Mittelwert NIFP $\square$ Mittelwert Keine Bewerbung

Abbildung 20: Bewertung der Informationsquellen im Bereich Wirtschaftsförderung (Eigene Darstellung). 
$\Xi$
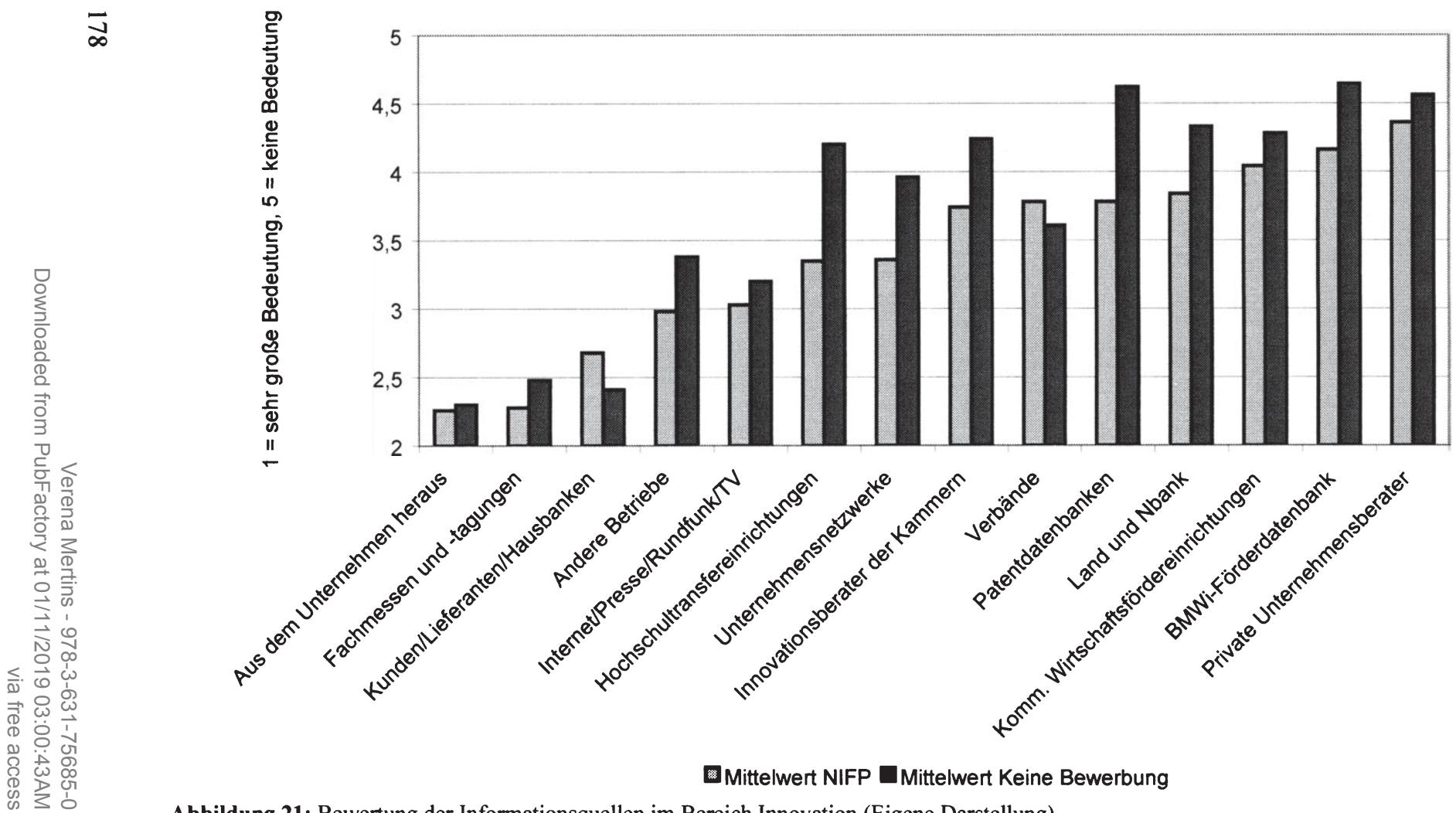

Mittelwert NIFP $\square$ Mittelwert Keine Bewerbung

Abbildung 21: Bewertung der Informationsquellen im Bereich Innovation (Eigene Darstellung). 
Einen Überblick über die Verteilung des Uninformiertheitsindikator nach Förderung bildet Abbildung 22. Es wird deutlich, dass Unternehmer, die sich nicht für ein Förderprogramm beworben haben, deutlich uninformierter sind als Unternehmer, die sich für ein Förderprogramm beworben haben. So liegt der Median von Unternehmern, die sich nicht für ein Förderprogramm beworben haben, bei 3,93, während er bei Unternehmern, Unternehmen, die Fördermittel aus anderen Programmen erhalten bzw. sich darum beworben haben, bei 3,75 und bei NIFPUnternehmern (gefördert oder beworben) bei 3,39 liegt.

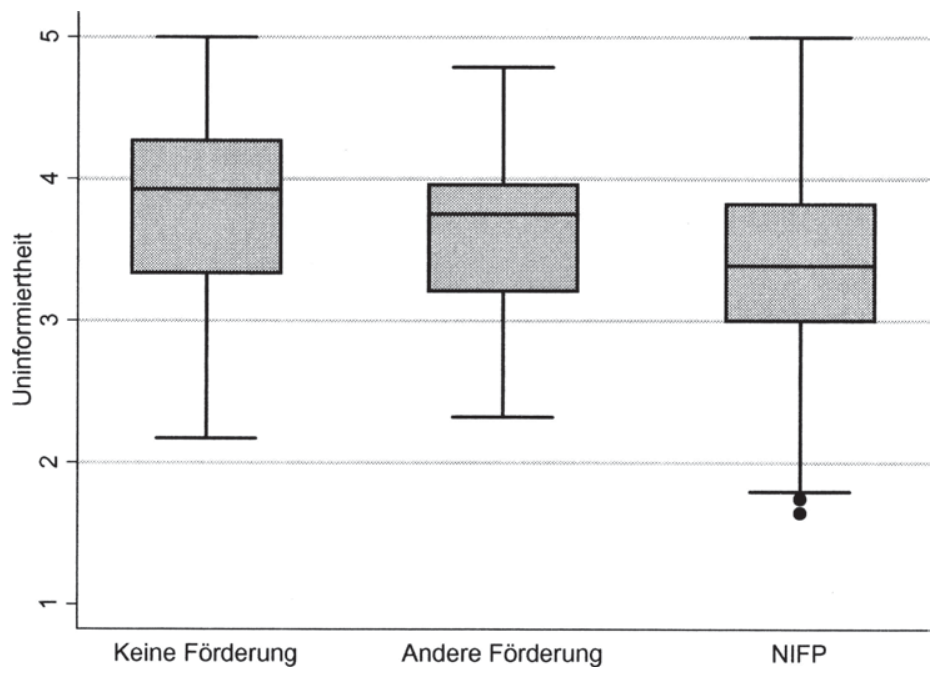

Abbildung 22: Uninformiertheit nach Förderung (Eigene Darstellung).

Der Kruskal-Wallis-Test bestätigt die Gruppenunterschiede bei einem Signifikanzniveau von $1 \%$. Die Prüfgröße des Tests liegt bei 22,79 (zwei Freiheitsgrade).

\subsubsection{Informationsverhalten bzgl. Wirtschaftsförderung}

Zentral für die Untersuchung der unternehmerischen Informiertheit in Bezug auf Wirtschaftsförderprogramme ist der erwähnte „Förderdschungel“: die vielen Unternehmen unübersichtlich erscheinende Vielfalt an Förderprogrammen unterschiedlichster Ausgestaltung für diverse Zielgruppen mit Mitteln aus den verschiedensten Fördertöpfen (vgl. Abschnitt 4.5.2). Zur Untersuchung dieses Phänomens sollten die befragten Unternehmer angeben, ob die Förderlandschaft ihrer Meinung nach übersichtlich oder unübersichtlich sei. Von den NIFPUnternehmern (gefördert oder beworben) bewerteten nur 56,07 \% die Förder- 
landschaft als unübersichtlich, während 77,27 \% der Unternehmer, die Fördermittel aus anderen Programmen erhalten bzw. sich darum beworben haben, und 95,24\% der Unternehmer, die sich nicht um Förderung beworben haben, die Förderlandschaft als unübersichtlich beurteilten. ${ }^{435}$ Dieses Ergebnis ist nicht sonderlich überraschend, da geförderte Unternehmer durch ihre Förderung schon bewiesen haben, dass sie mit dem vorliegenden Informationsdschungel gut zurechtkommen - schließlich haben sie ja ein geeignetes Förderprogramm gefunden.

Es stellt sich nun jedoch die Frage, ob dieser Erfolg das Ergebnis einer strategischen Informationssuche und besser ausgebildeten Informationskompetenz darstellt. Im Folgenden wird deshalb untersucht, ob Unternehmer, die sich in der Vergangenheit für Förderprogramme beworben haben, die im Bereich Wirtschaftsförderung vorhandenen Informationsquellen intensiver nutzen als Unternehmer, die sich nicht für derartige Programme beworben haben. Infolgedessen könnten Unternehmer der ersten Gruppe tatsächlich als informierter bezeichnet werden als Unternehmer der zweiten Gruppe.

Tatsächlich weisen NIFP-Unternehmer (gefördert oder beworben) nicht nur elf von vierzehn angegebenen Informationsquellen im Bereich Wirtschaftsförderung eine stärkere Bedeutung $\mathrm{zu}$ als nicht geförderte Unternehmer, sondern sie bewerten bestimmte Informationsquellen auch als besonders wichtig, um sich über Wirtschaftsförderung zu informieren, die die nicht geförderten Unternehmer als nicht so wichtig ansehen. Die Mittelwerte und Mediane der Bewertung sind in Tabelle 7 dargestellt. Bei den von den geförderten Unternehmern als besonders wichtig bewerteten Informationsquellen handelt es sich um die Informationsschriften bzw. die Internetseite des Landes Niedersachsen und der NBank, die kommunalen Wirtschaftsfördereinrichtungen, die Innovationsberater der Kammern und die Förderdatenbank des Bundesministeriums für Wirtschaft und Technologie (BMWi). Es ist davon auszugehen, dass genau diese Informationsquellen von besonderer Bedeutung für die erfolgreiche Suche nach Innovationsförderprogrammen sind. So schätzen Unternehmer, die sich für ein Förderprogramm beworben haben, diese Quellen als sehr wichtig ein. Es handelt sich bei diesen Quellen um spezielle zielführende Quellen, die im Rahmen einer strategischen Suche nach Informationen verwendet werden. Dagegen sind die einzigen Quellen, die von Unternehmern, die sich nicht für ein Förderprogramm beworben haben, als wichtiger bewertet werden, im Allgemeinen einfacher zugänglich (Fachmessen und -tagungen, Informationen der Verbände sowie Kunden, Lieferanten und Hausbanken). Doch werden diese Quellen nicht im Rahmen einer

435 Diese Prozentzahlen beziehen sich auf diejenigen, die die Frage beantwortet haben. Von den durch das NIFP geförderten Unternehmen haben 92,62 \% diese Frage beantwortet. Bei den durch andere Wirtschaftsförderprogramme geförderten Unternehmen waren es $88,46 \%$, bei den nicht geförderten Unternehmen 69,15\%. Danach wurden diejenigen Fragebögen, die nicht vom Unternehmer selbst ausgefüllt wurden, aussortiert. 
strategischen Suche genutzt, sondern führen nur zufällig zu hilfreichen Anregungen. ${ }^{436}$

Es wurden die drei Gruppen der nicht geförderten, der durch das NIFP geförderten und der durch andere Wirtschaftsförderprogramme geförderten Unternehmen mit Hilfe des Kruskal-Wallis-Tests hinsichtlich ihrer Bewertung der Wichtigkeit der verschiedenen Informationsquellen miteinander verglichen (vgl. Tabelle 7). ${ }^{437}$ Die Differenzen in der Bewertung sind für folgende acht Quellen im Bereich Wirtschaftsförderung signifikant: Informationsschriften/Internetseite des Landes Niedersachsen und der NBank, BMWi-Förderdatenbank, kommunale Wirtschaftsfördereinrichtungen, Innovationsberater der Kammern, Patentdatenbanken, Hochschultransfereinrichtungen, Unternehmensnetzwerke (jeweils auf dem $1 \%$-Niveau) sowie private Unternehmensberater (auf dem $5 \%$ Niveau). ${ }^{438}$

Um die Gesamtheit der angegebenen Informationsquellen für den Bereich Wirtschaftsförderung zu berücksichtigen, wird ein Informiertheitsindikator nur für diese Quellen gebildet, der den Mittelwert der Bewertung durch den Unternehmer darstellt. Beim Vergleich der Ausprägungen dieses Informiertheitsindikators für die verschiedenen Unternehmergruppen anhand von Abbildung 23 wird deutlich, dass NIFP-Unternehmer (gefördert oder beworben) deutlich besser informiert sind (Median von 3,36) als Unternehmer, die sich nicht um Förderung beworben haben (Median von 3,97), sowie Unternehmer, die Fördermittel aus anderen Programmen erhalten bzw. sich darum beworben haben (Median von 3,68). Der Kruskal-Wallis-Test bestätigt die Differenzen zwischen den Gruppen bei einem Signifikanzniveau von $1 \%$. Die Prüfgröße nimmt den Wert von 23,99 an (zwei Freiheitsgrade).

436 Vgl. mit einer ähnlichen Argumentation Förderer et al. (1998), S. 12.

437 Da die Bewertung der Informationsquellen mit einer Skala von 1 bis 5 vorgenommen wurde, handelt es sich bei der Bewertung um eine ordinal skalierte Variable, so dass der Gruppenvergleich mit Hilfe des Kruskal-Wallis-Tests erfolgt. - Die Differenzen zwischen geförderten und nicht geförderten Unternehmen verstärken sich noch, wenn nur die beiden Gruppen der NIFP-Unternehmer und der Unternehmer, die sich nicht um Förderung beworben haben, mit Hilfe des Wilcoxon-Rangsummentests verglichen werden oder wenn die Gruppe der Unternehmer, die Fördermittel aus dem NIFP oder aus anderen Förderprogrammen erhalten haben oder sich darum beworben haben, mit den Unternehmern, die sich überhaupt nicht um Förderung beworben haben, verglichen werden.

438 Auch die oben erläuterte bessere Bewertung der nicht geförderten Unternehmer der Informationsquellen Kunden, Lieferanten und Hausbanken ist auf dem $1 \%$-Niveau signifikant. 
Tabelle 7: Informationsquellen für Wirtschaftsförderung differenziert nach Förderarten

\begin{tabular}{|c|c|c|c|c|c|c|c|c|}
\hline $\begin{array}{l}\text { Informationsquellen für } \\
\text { Wirtschaftsförderung }\end{array}$ & $\begin{array}{c}\text { NIFP- } \\
\text { Förderung } \\
\text { (Mittelwert) }\end{array}$ & $\begin{array}{c}\text { Andere } \\
\text { Förderung } \\
\text { (Mittelwert) }\end{array}$ & $\begin{array}{c}\text { Keine } \\
\text { Bewerbung } \\
\text { (Mittelwert) }\end{array}$ & $\begin{array}{l}\text { NIFP- } \\
\text { Förderung } \\
\text { (Median) }\end{array}$ & $\begin{array}{l}\text { Andere } \\
\text { Förderung } \\
\text { (Median) }\end{array}$ & $\begin{array}{c}\text { Keine } \\
\text { Bewerbung } \\
\text { (Median) }\end{array}$ & $\begin{array}{l}\text { Kruskal- } \\
\text { Wallis- } \\
\text { Prüfgröße } \\
(2 \mathrm{FG})\end{array}$ & $\operatorname{Pr}$ \\
\hline $\begin{array}{l}\text { Informationsschriften/Internetseite } \\
\text { des Landes Niedersachsens und der } \\
\text { NBank }\end{array}$ & 2,69 & 3,50 & 4,09 & 3 & 3,5 & 5 & 52,64 & $0,00 * * *$ \\
\hline Aus dem Unternehmen heraus & 2,97 & 3,08 & 3,38 & 3 & 3 & 3 & 3,97 & 0,14 \\
\hline $\begin{array}{l}\text { Kommunale } \\
\text { Wirtschaftsfördereinrichtungen }\end{array}$ & 3,01 & 3,57 & 4,01 & 3 & 4 & 4 & 30,61 & $0,00 * * *$ \\
\hline Internet/Presse/Rundfunk/TV & 3,15 & 3,21 & 3,47 & 3 & 3 & 3 & 2,91 & 0,23 \\
\hline Innovationsberater der Kammern & 3,17 & 4,04 & 4,00 & 4 & 5 & 5 & 18,94 & $0,00 * * *$ \\
\hline Fachmessen/-tagungen & 3,24 & 3,17 & 3,03 & 3 & 3 & 3 & 1,37 & 0,50 \\
\hline BMWi-Förderdatenbank & 3,38 & 4,00 & 4,41 & 3 & 4 & 5 & 40,39 & $0,00 * * *$ \\
\hline Unternehmensnetzwerke & 3,45 & 3,83 & 4,12 & 3 & 4 & 4 & 14,36 & $0,00 * * *$ \\
\hline Hochschultransfereinrichtungen & 3,53 & 4,45 & 4,36 & 4 & 5 & 5 & 32,02 & $0,00 * * *$ \\
\hline Kunden/Lieferanten/Hausbanken & 3,58 & 3,17 & 3,01 & 4 & 3 & 3 & 9,54 & $0,01 * * *$ \\
\hline Andere Betriebe & 3,64 & 3,46 & 3,81 & 4 & 3 & 4 & 2,09 & 0,35 \\
\hline Verbände & 3,70 & 3,71 & 3,60 & 4 & 4 & 4 & 0,18 & 0,91 \\
\hline Private Unternehmensberater & 4,03 & 4,29 & 4,49 & 5 & 5 & 5 & 8,22 & $0,02 * *$ \\
\hline Patentdatenbanken & 4,07 & 4,57 & 4,63 & 4 & 5 & 5 & 18,54 & $0,00 * * *$ \\
\hline
\end{tabular}

*10\%,**5\%,***1\%.

Quelle: Eigene Auswertung. 


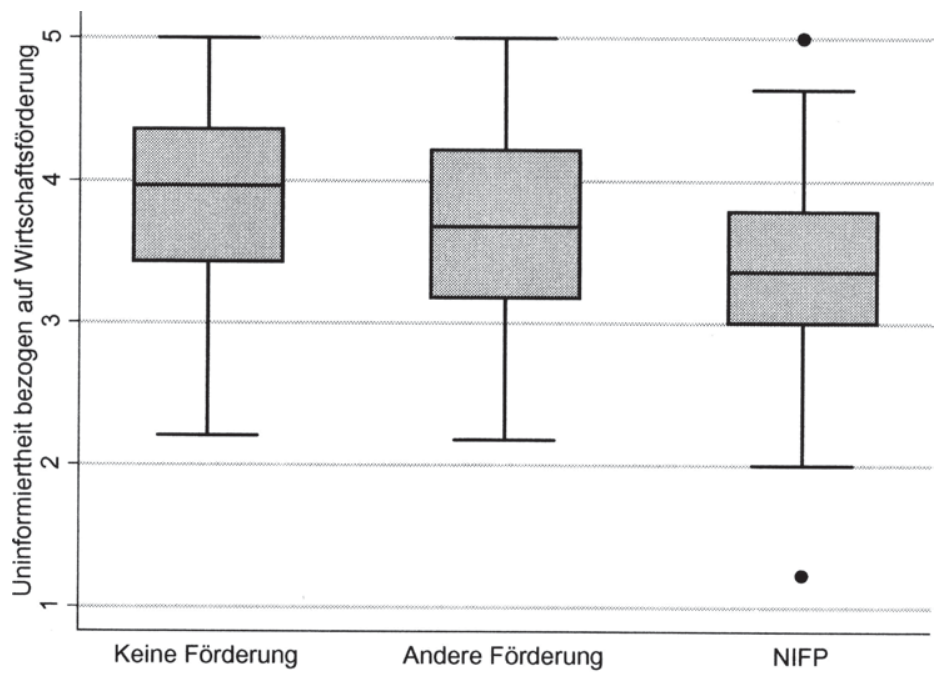

Abbildung 23: Uninformiertheit bezogen auf Wirtschaftsförderung (Eigene Darstellung).

Bei Wahl des Unternehmens anstelle des Unternehmers als Bezugspunkt für das Informiertheitskonzept (vgl. hierzu Abschnitt 5.4.5) ergibt sich die Möglichkeit, die Informiertheit im Bereich Wirtschaftsförderung mit Hilfe der Mitarbeiterzuständigkeit für die Suche nach für das Unternehmen passenden Förderprogrammen zu messen. Wenn es einen dafür zuständigen Mitarbeiter im Unternehmen gibt, dann kümmert sich dieser Verantwortliche aktiv um die Informationssuche und fungiert gleichzeitig passiv als Informationsanlaufstelle im Unternehmen. Deswegen ist ein Unternehmen mit festgelegter personeller Zuständigkeit in der Regel besser informiert im Bereich Wirtschaftsförderung als ein Unternehmen ohne einen dafür zuständigen Mitarbeiter. In 50,82\% der Unternehmen, die durch das NIFP unterstützt werden oder sich um eine Unterstützung erfolglos beworben haben, gibt es einen Verantwortlichen, der für die Suche nach Wirtschaftsförderprogrammen zuständig ist, im Gegensatz zu nur 20,21 \% der nicht geförderten und zu 53,85\% der durch andere Wirtschaftsförderprogramme unterstützten Unternehmen. ${ }^{439}$ In 68,42 \% aller Unternehmen mit personeller $\mathrm{Zu}$ ständigkeit hat der Geschäftsführer bzw. Unternehmensinhaber diese Funktion inne. In größeren Unternehmen fungiert oftmals die Leitung der Controllingoder der F\&E-Abteilung als Ansprechpartner. In den Unternehmen ohne festgelegte personelle Zuständigkeit ist davon auszugehen, dass sich der Unternehmer

439 Von den nicht geförderten Unternehmen haben 2,13 \% die Frage nicht beantwortet. Bei den durch das NIFP geförderten Unternehmen waren es 1,64 \% und bei den durch andere Förderprogramme geförderten Unternehmen $0 \%$. 
überwiegend selbst um die Suche nach für das Unternehmen in Frage kommenden Wirtschaftsförderprogrammen kümmert - sofern er neben seiner gewöhnlichen Geschäftstätigkeit Zeit dafür findet.

\subsubsection{Informiertheit in Bezug auf den Bewerbungsaufwand bei Innovationsförderprogrammen}

Wie der vorherige Abschnitt gezeigt hat, sind nicht geförderte Unternehmer im Durchschnitt schlechter über Förderprogramme und deren Ablauf und Bewerbungsmodalitäten informiert als Unternehmer, die sich in der Vergangenheit für ein Förderprogramm beworben haben. Da nun die Möglichkeit besteht, dass Unternehmer, die sich in der Vergangenheit nicht um Förderung beworben haben, aufgrund ihrer schlechteren Informiertheit auch den mit einer Bewerbung verbundenen Aufwand überschätzen und sich deswegen nicht für Innovationsförderprogramme bewerben, wurde in Abschnitt 5.4 .5 bezogen auf das NIFP die Hypothese 8 aufgestellt, dass der Bewerbungs- und Dokumentationsaufwand in der Realität nicht so groß ist wie in der Vorstellung derjenigen Unternehmer, die sich nicht für das NIFP beworben haben.

Die Ergebnisse der Befragung unterstützen diese Hypothese allerdings nicht. 96,67\% der Unternehmer, die sich nicht um Förderung beworben haben, halten die Antragstellung bei Innovationsförderprogrammen zwar generell für aufwendig. Dieser Prozentsatz liegt erheblich höher als bei den durch NIFPUnternehmern (40,91\%) und den Unternehmern, die sich für andere Programme beworben haben $(86,96 \%) .{ }^{440}$ Beim Vergleich des tatsächlich geleisteten Bewerbungsaufwands und der Vorstellung des zu leistenden Bewerbungsaufwands seitens der in der Förderung unerfahrenen Unternehmer zeigen sich bemerkenswerte Ergebnisse. Obwohl die in der Förderung unerfahrenen Unternehmer die Antragstellung generell für aufwendig halten, haben sie eine viel zu geringe Vorstellung vom tatsächlichen mit der Antragstellung verbundenen Arbeitsaufwand.

Die folgenden Boxplots illustrieren den Unterschied zwischen Realität und Vorstellung. Die Unternehmer, die in der Vergangenheit Förderung aus dem NIFP erhalten bzw. sich darum beworben haben, wurden befragt, welcher Zeitaufwand in ihrem Unternehmen insgesamt dafür benötigt wurde (gemessen in Personenstunden), Antragskizze, Förderantrag und Dokumentation der Fördermittelverwendung zu erstellen. Die anderen Unternehmergruppen wurden nach ihrer Einschätzung des jeweiligen Zeitaufwands befragt (vgl. Frage 18 des Fragebogens für nicht geförderte Unternehmen in Anhang D).

440 Diese Zahlen beziehen sich auf diejenigen, die die Frage zur Antragstellung beantwortet haben. Von den nicht geförderten Unternehmen haben $68,09 \%$ die Frage beantwortet. Bei den durch das NIFP geförderten Unternehmen waren es $95,08 \%$ und bei den durch andere Förderprogramme geförderten Untermehmen 92,31\%. 


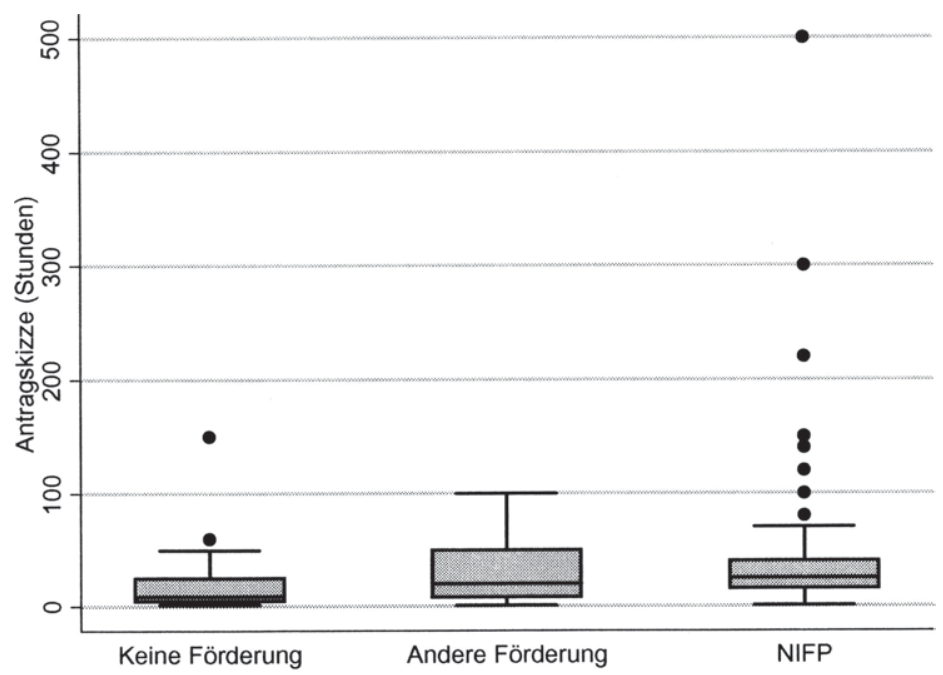

Abbildung 24: Zeitaufwand für die Erstellung der Antragskizze (Eigene Darstellung).

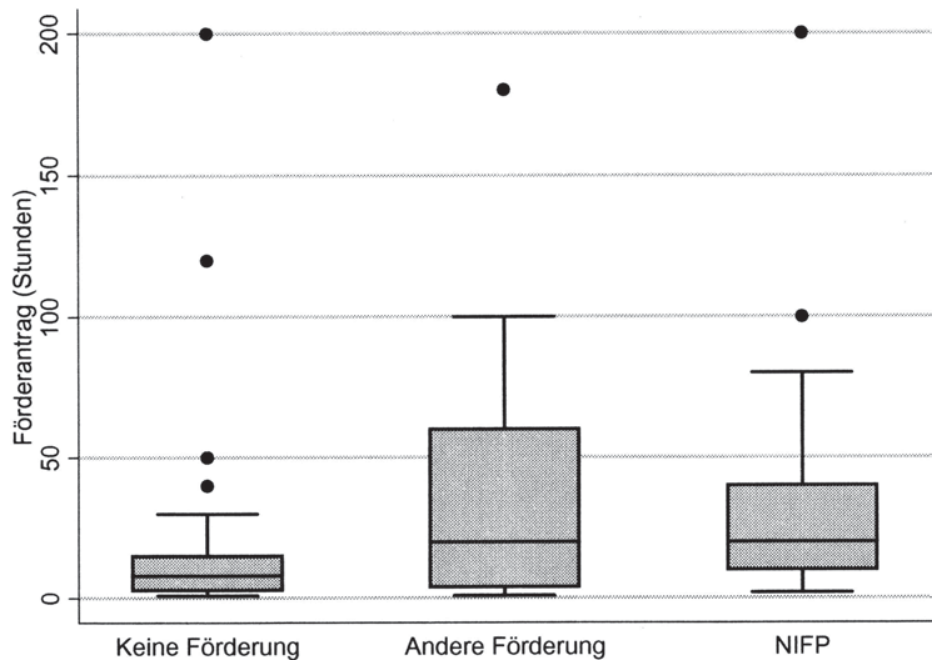

Abbildung 25: Zeitaufwand für die Erstellung des Förderantrags (Eigene Darstellung). 


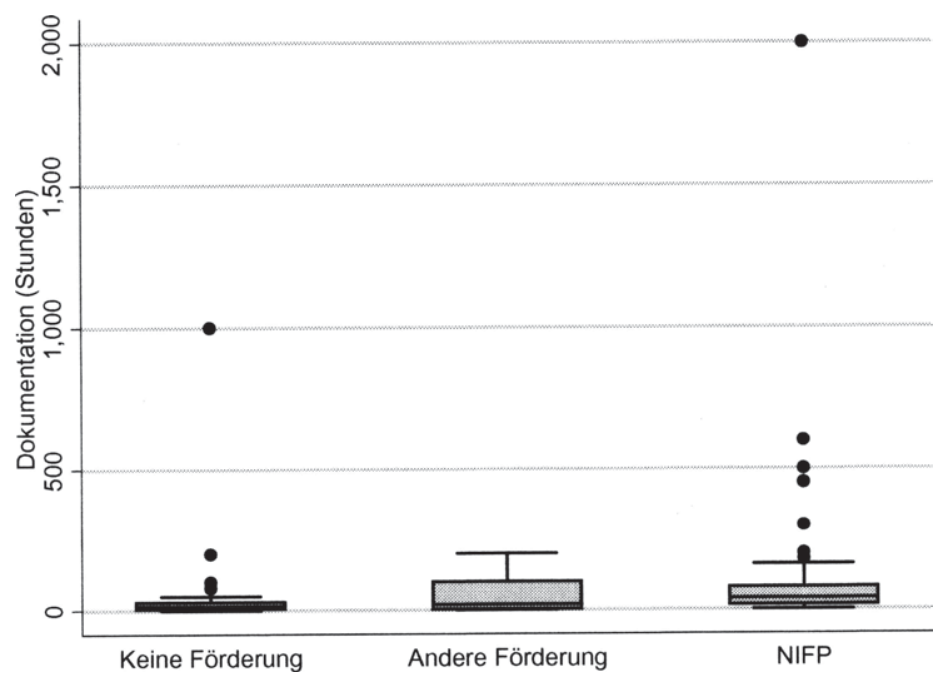

Abbildung 26: Zeitaufwand für die Erstellung der Dokumentation der Fördermittelverwendung (Eigene Darstellung).

Tabelle 8 stellt die Mittelwerte und Mediane des angegebenen Zeitaufwands der drei nach Förderarten differenzierten Unternehmensgruppen dar. Die Werte unterscheiden sich deutlich voneinander. Die Unternehmer, die keine Erfahrung mit Innovationsförderprogrammen besitzen, unterschätzen den anfallenden Bewerbungs- und Dokumentationsaufwand deutlich, während die Unternehmen, die Fördermittel aus anderen Programmen erhalten bzw. sich darum beworben haben, mit ihrer Einschätzung deutlich näher an der Realität liegen. Dies hängt höchstwahrscheinlich mit der Erfahrung dieser Unternehmen den Bewerbungsablauf solcher Programme betreffend zusammen.

Tabelle 8: Zeitaufwand für die Bewerbung und Dokumentation

\begin{tabular}{lcccccc}
\hline $\begin{array}{l}\text { Zeitaufwand } \\
\text { für ... }\end{array}$ & $\begin{array}{c}\text { NIFP- } \\
\text { Förderung } \\
\text { (Mittelwert) }\end{array}$ & $\begin{array}{c}\text { Andere } \\
\text { Förderung } \\
\text { (Mittelwert) }\end{array}$ & $\begin{array}{c}\text { Keine } \\
\text { Bewerbung } \\
\text { (Mittelwert) }\end{array}$ & $\begin{array}{c}\text { NIFP- } \\
\text { Förderung } \\
\text { (Median) }\end{array}$ & $\begin{array}{c}\text { Andere } \\
\text { Förderung } \\
\text { (Median) }\end{array}$ & $\begin{array}{c}\text { Keine } \\
\text { Bewerbung } \\
\text { (Median) }\end{array}$ \\
\hline Antragskizze & 39,20 & 27,89 & 19,67 & 25 & 20 & 9 \\
$\begin{array}{l}\text { Förderantrag } \\
\begin{array}{l}\text { Dokumentation } \\
\text { der Fördermit- } \\
\text { telverwendung }\end{array}\end{array}$ & 36,45 & 38,79 & 15,55 & 20 & 20 & 8 \\
\hline
\end{tabular}

Quelle: Eigene Auswertung. 
Der Wilcoxon-Rangsummentest bestätigt die Signifikanz der Mittelwertunterschiede zwischen Unternehmen, die sich für das NIFP beworben haben und Unternehmen, die sich für kein Wirtschaftsförderprogramm beworben haben, in Bezug auf den Zeitaufwand für die Antragskizze, den Förderantrag und die Dokumentation der Fördermittelverwendung auf dem 1 \%-Niveau.

Tabelle 9: Ergebnisse des Wilcoxon-Rangsummentest für den Zeitaufwand

\begin{tabular}{lcc}
\hline Zeitaufwand für ... & Mann-Whitney-U-Prüfgröße Z-Wert & p-Wert \\
\hline Antragskizze & $-3,99$ & $0,00 * * *$ \\
Förderantrag & $-6,08$ & $0,00 * * *$ \\
Dokumentation der & $-4,24$ & $0,00 * * *$ \\
\hline Fördermittelverwendung & & \\
\hline
\end{tabular}

$* 10 \%, * * 5 \%, * * * 1 \%$.

Quelle: Eigene Auswertung.

Der hohe Bewerbungsaufwand überfordert die Unternehmen zum Teil, was sich daran erkennen lässt, dass $31,97 \%$ der geförderten Unternehmen externe Hilfe bei der Antragstellung in Anspruch genommen haben. ${ }^{441}$ Diese externe Hilfe war zu 37,5\% kostenlos, der Mittelwert der Kosten liegt bei $5053 €$, der Maximalwert bei $25.000 € €^{442}$

\subsubsection{Informationsverhalten bzgl. Innovation}

Während sich die Informiertheit in Bezug auf Wirtschaftsförderung von Unternehmern, die sich in der Vergangenheit um Förderung beworben haben, und Unternehmern, die sich nicht beworben haben, stark unterscheidet, werden Informationsquellen zum Erhalt von Anregungen für Innovationen sehr ähnlich eingeschätzt. Dabei werden insgesamt nur innovativ tätige Unternehmer betrachtet und nach Förderung differenziert, weil anzunehmen ist, dass innovative Unternehmer Informationsquellen zur Anregung für Innovationen generell als wichtiger bewerten als nicht innovativ tätige Unternehmer, die keine Anregungen für Innovationen suchen. Dieses Ergebnis wird durch die empirische Evidenz für Handwerksunternehmen von Astor et al. (2006) gestützt. ${ }^{443}$

Die Ranglisten der Bewertung der Informationsquellen sind fast identisch. Sowohl NIFP-Unternehmer (gefördert oder beworben) als auch Unternehmer, die sich nicht um Förderung beworben haben, bewerten die folgenden Informa-

$44159,84 \%$ nahmen keine externe Hilfe in Anspruch und 8,20\% machten keine Angabe.

442 Von den Unternehmen, die angaben, externe Hilfe in Anspruch genommen zu haben, gaben $82 \%$ die exakte Kostenhöhe für die externe Hilfe an.

443 Vgl. Astor et al. (2006), S. 69 f. 
tionsquellen als am wichtigsten: Anregungen aus dem Unternehmen heraus, Fachmessen/-tagungen sowie Kunden, Lieferanten und Hausbanken. Unterschiede ergeben sich in der Bewertung von Patentdatenbanken, Hochschultransfereinrichtungen und Unternehmensnetzwerken, welche von Unternehmern, die sich nicht um Förderung beworben haben, als deutlich weniger wichtig eingeschätzt werden als von NIFP-Unternehmern (vgl. Tabelle 10). Auch hier wird also deutlich, dass Unternehmer, die sich um Förderung beworben haben, informierter sind als Unternehmer, die sich nicht um Förderung beworben haben. Die erste Unternehmergruppe misst zwölf von vierzehn angegebenen Informationsquellen eine höhere Bedeutung zu als die zweite Gruppe. Nur Informationen der Verbände sowie Kunden, Lieferanten und Hausbanken werden von Unternehmern, die sich nicht um Förderung beworben haben, als wichtiger bewertet als von Unternehmern, die Fördermittel aus dem NIFP erhielten bzw. sich darum beworben haben. Von den Differenzen in der Bewertung der zwölf Informationsquellen, denen NIFP-Unternehmer eine höhere Bedeutung beimessen, sind bei Betrachtung aller drei nach Förderung differenzierten Unternehmergruppen die sieben Abweichungen für folgende Informationsquellen bei einem Niveau von $1 \%, 5 \%$ bzw. $10 \%$ signifikant: Unternehmensnetzwerke, Hochschultransfereinrichtungen, Patentdatenbanken, Innovationsberater der Kammern, Informationsschriften/Internetseite des Landes Niedersachsen und der NBank, Anregungen durch andere Betriebe und die BMWi-Förderdatenbank.

Die Differenzen zwischen den Unternehmergruppen werden noch deutlicher bei einem Vergleich nur der beiden Gruppen der NIFP-Unternehmer und der Unternehmer, die sich nicht um Förderung beworben haben, mit Hilfe des Wilcoxon-Rangsummentests (vgl. Tabelle 18 in Anhang J).

Analog zum Vorgehen für den Bereich der Wirtschaftsförderung wird auch hier ein Informiertheitsindikator nur für die Quellen des Bereichs Innovation gebildet, der den Mittelwert der Bewertung durch den Unternehmer darstellt. Beim Vergleich der Ausprägungen dieses Informiertheitsindikators für geförderte und nicht geförderte Unternehmen anhand von Abbildung 27 wird deutlich, dass die durch das NIFP geförderten Unternehmer deutlich besser informiert sind (Median von 3,43) als nicht geförderte Unternehmer (Median von 3,79) bzw. Unternehmer, die sich für andere Förderprogramme beworben haben (Median von 3,47). Jedoch sind die Differenzen für Informationsquellen des Bereichs Innovation nicht ganz so deutlich wie für den Bereich Wirtschaftsförderung. Der Kruskal-Wallis-Test bestätigt die Differenzen zwischen den Gruppen bei einem Signifikanzniveau von $1 \%$. Die Prüfgröße nimmt den Wert von 13,79 an (zwei Freiheitsgrade). 
Tabelle 10: Informationsquellen für Innovation differenziert nach Förderarten nur für innovativ tätige Unternehmen

\begin{tabular}{|c|c|c|c|c|c|c|c|c|}
\hline Informationsquellen für Innovation & $\begin{array}{c}\text { NIFP } \\
\text { (Mittelwert) }\end{array}$ & $\begin{array}{c}\text { Andere } \\
\text { Förderung } \\
\text { (Mittelwert) }\end{array}$ & $\begin{array}{c}\text { Keine } \\
\text { Bewerbung } \\
\text { (Mittelwert) }\end{array}$ & $\begin{array}{c}\text { NIFP } \\
\text { (Median) }\end{array}$ & $\begin{array}{l}\text { Andere } \\
\text { Förderung } \\
\text { (Median) }\end{array}$ & $\begin{array}{c}\text { Keine } \\
\text { Bewerbung } \\
\text { (Median) }\end{array}$ & $\begin{array}{l}\text { Kruskal- } \\
\text { Wallis- } \\
\text { Prüfgröße } \\
(2 \mathrm{FG})\end{array}$ & Pr \\
\hline Aus dem Unternehmen heraus & 2,26 & 2,17 & 2,30 & 2 & 2 & 2 & 0,50 & 0,78 \\
\hline Fachmessen/-tagungen & 2,28 & 2,29 & 2,48 & 2 & 2 & 3 & 1,77 & 0,41 \\
\hline Kunden/Lieferanten/Hausbanken & 2,68 & 2,54 & 2,41 & 2 & 2 & 2 & 1,27 & 0,53 \\
\hline Andere Betriebe & 2,98 & 2,83 & 3,38 & 3 & 3 & 3 & 4,72 & $\begin{array}{r}0,09 \\
* \quad 5\end{array}$ \\
\hline Internet/Presse/Rundfunk/Fernsehen & 3,03 & 2,88 & 3,20 & 3 & 3 & 3 & 1,03 & 0,60 \\
\hline Hochschultransfereinrichtungen & 3,35 & 4,05 & 4,20 & 3 & 4,5 & 5 & 18,22 & $0,00 * * *$ \\
\hline Unternehmensnetzwerke & 3,36 & 3,29 & 3,96 & 3 & 3 & 4 & 8,30 & $0,02 * *$ \\
\hline Innovationsberater der Kammern & 3,74 & 4,50 & 4,24 & 4 & 5 & 5 & 12,83 & $0,00 * * *$ \\
\hline Verbände & 3,78 & 3,58 & 3,61 & 4 & 3 & 4 & 0,75 & 0,69 \\
\hline $\begin{array}{l}\text { Patentdatenbanken } \\
\text { Informationsschriften/Internetseite }\end{array}$ & 3,78 & 4,48 & 4,62 & 4 & 5 & 5 & 24,90 & $0,00 * * *$ \\
\hline $\begin{array}{l}\text { des Landes Niedersachsen und der } \\
\text { NBank }\end{array}$ & 3,84 & 4,17 & 4,33 & 4 & 5 & 5 & 10,48 & $0,01 * * *$ \\
\hline $\begin{array}{l}\text { Kommunale Wirtschaftsförder- } \\
\text { einrichtungen }\end{array}$ & 4,04 & 4,13 & 4,28 & 4 & 4 & 5 & 2,47 & 0,29 \\
\hline BMWi-Förderdatenbank & 4,16 & 4,38 & 4,64 & 4 & 5 & 5 & 10,48 & $0,01 * * *$ \\
\hline Private Unternehmensberater & 4,36 & 4,25 & 4,56 & 5 & 5 & 5 & 2,45 & 0,29 \\
\hline
\end{tabular}

* $10 \%$,**5\%,***1\%.

Quelle: Eigene Auswertung. 


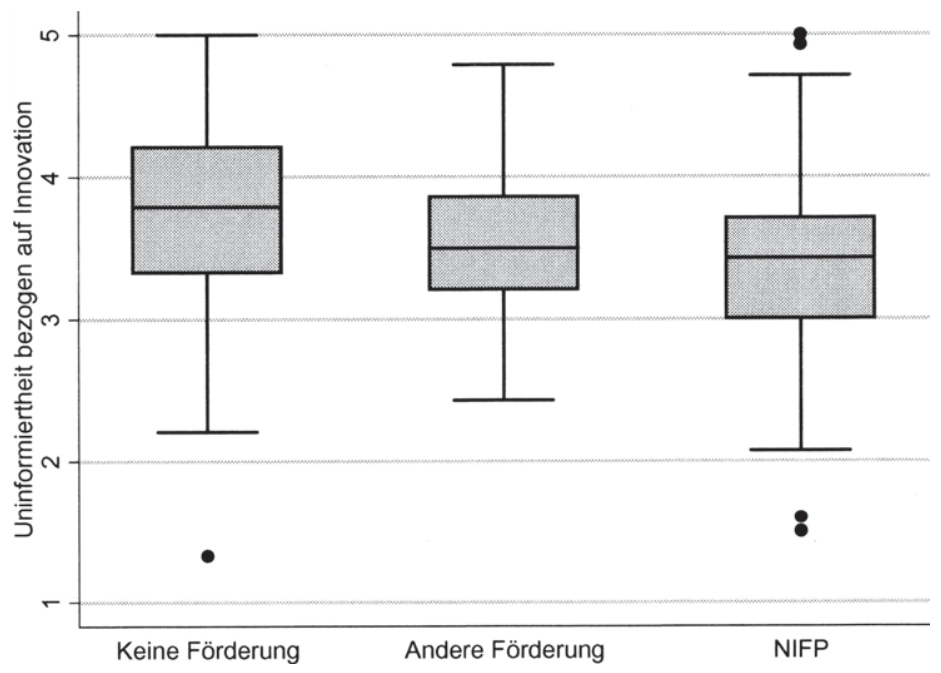

Abbildung 27: Uninformiertheit bezogen auf den Bereich Innovation (Eigene Darstellung).

\subsubsection{Informiertheit der befragten Handwerksunternehmen}

Neben der Förderung scheint die Kammerzugehörigkeit eine Auswirkung auf die Informiertheit eines Unternehmens zu haben. Es fällt auf, dass die Bewertungen der Informationsquellen von Unternehmern aus Handwerks- und Mischbetrieben gegenüber Unternehmern aus Industriebetrieben teilweise deutlich voneinander abweichen. Die schon in Abschnitt 4.2. erwähnte wichtige Funktion der Innovationsberater der Handwerkskammern wird durch die Bewertung der NIFP-Unternehmer (gefördert und beworben) gestützt. Die Innovationsberater stellen die mit Abstand wichtigste Informationsquelle für die NIFP-Unternehmer in Bezug auf Wirtschaftsförderung dar, wie in Abbildung 28 deutlich wird, welche die Bewertung der Informationsquellen für den Bereich Wirtschaftsförderung für Unternehmer aus Handwerks- und Mischbetrieben darstellt. Auch um Anregungen für Innovationen zu erhalten, werden Innovationsberater von diesen Unternehmern als deutlich wichtiger eingeschätzt gegenüber der Gesamtheit der befragten Unternehmer, wie aus Abbildung 29 im Vergleich zu Abbildung 21 deutlich wird. Dieses Ergebnis deckt sich mit den Erkenntnissen der Studie des RWI (2004), die zeigt, dass Handwerksunternehmen die Informationsangebote von Kammern stärker als andere Unternehmen nutzen, um sich über Innovationen zu informieren. ${ }^{444}$

444 Vgl. RWI (2004), S. 44 f. 

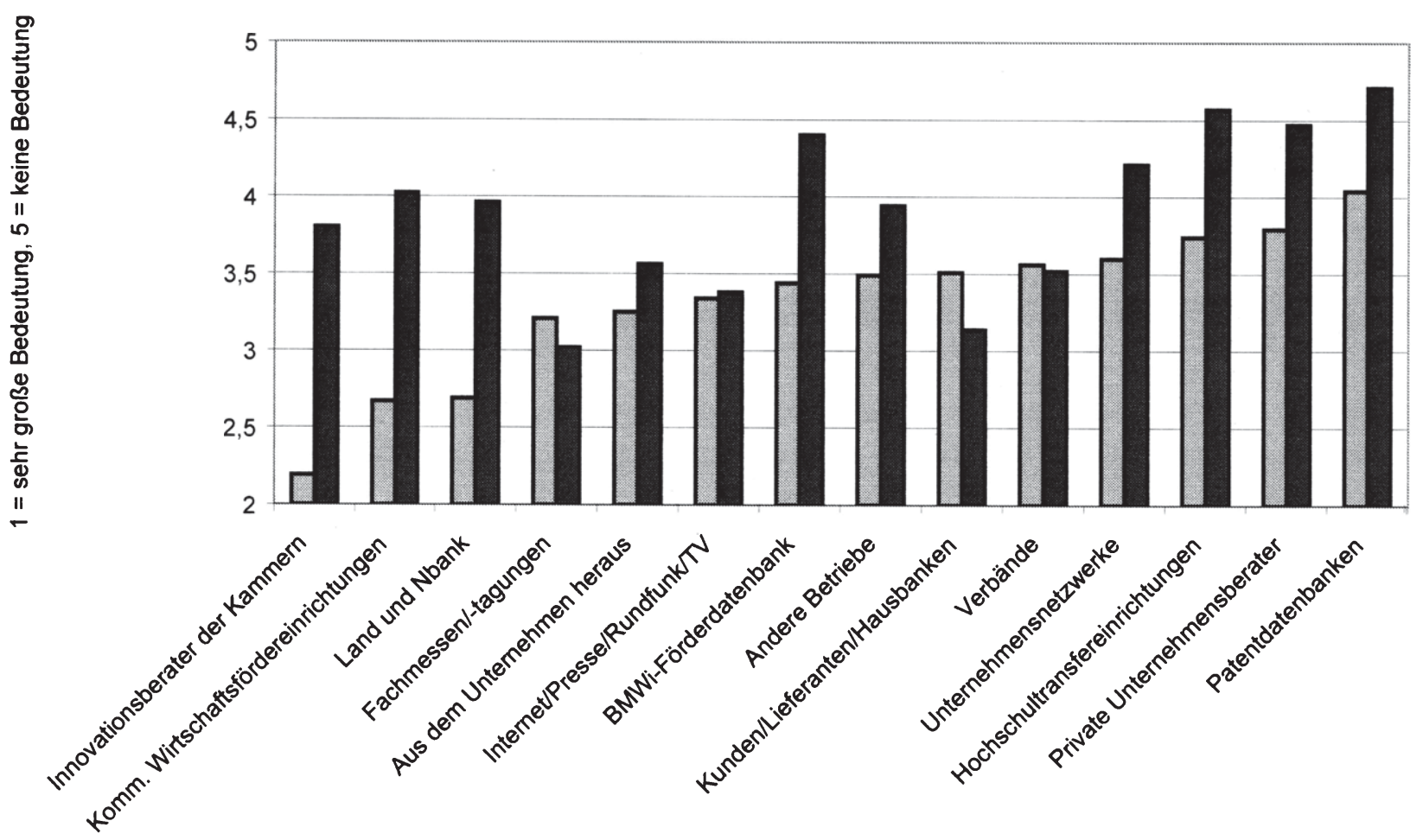

Mittelwert NIFP $\square$ Mittelwert Keine Förderung

Abbildung 28: Bewertung der Informationsquellen im Bereich Wirtschaftsförderung - nur Handwerksunternehmen (Eigene Darstellung). 
is
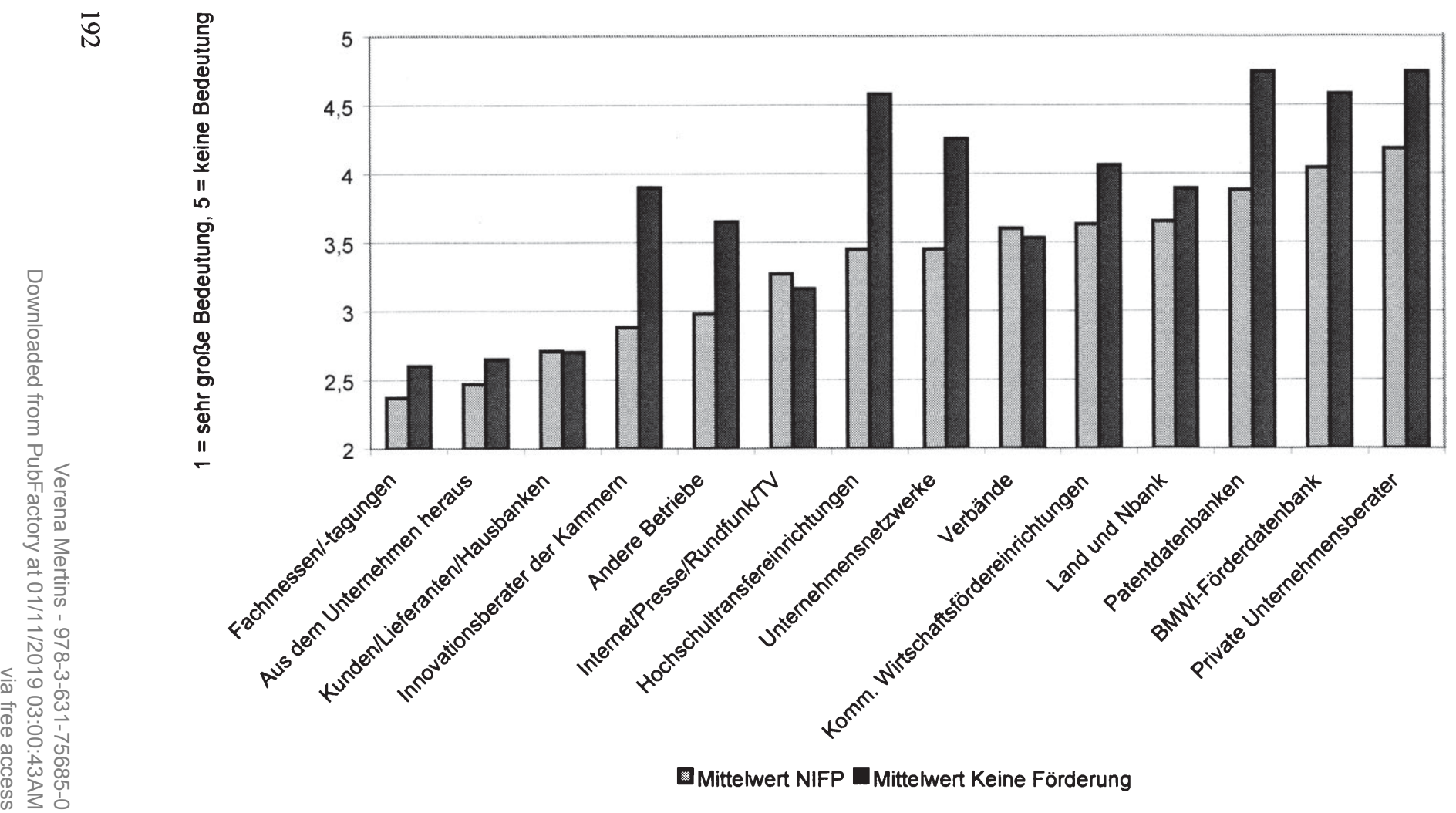

Mittelwert NIFP $\square$ Mittelwert Keine Förderung

Abbildung 29: Bewertung der Informationsquellen im Bereich Innovation - nur Handwerksunternehmen (Eigene Darstellung). 
Bei den anderen abgefragten Informationsquellen ergeben sich dagegen weder im Bereich Wirtschaftsförderung noch im Bereich Innovation wesentliche Unterschiede in der Bewertung zwischen Unternehmern aus Handwerks- und Mischbetrieben und der Gesamtheit der befragten Unternehmer.

\subsubsection{Zusammenfassung der Ergebnisse}

Die Ergebnisse der Unternehmensbefragung weisen auch in Bezug auf die Informiertheit des Unternehmers auf wesentliche Unterschiede zwischen geförderten und nicht geförderten Unternehmern hin. Unternehmer, die Förderung erhalten bzw. sich dafür bewerben, nutzen fast alle Informationsquellen in den Bereichen Wirtschaftsförderung und Innovation intensiver als Unternehmer, die sich nicht um Förderung bewerben. Die Uninformiertheit der Unternehmer, die sich nicht um Förderung bewerben, - gemessen mit einem aus der Bewertung der einzelnen Informationsquellen gebildeten Informiertheitsindikator - ist signifikant höher als die Uninformiertheit der Unternehmer, die sich für ein Förderprogramm beworben haben. Die Gruppe der NIFP-Unternehmer (gefördert oder beworben) sind bezogen auf die Gebiete Wirtschaftsförderung und Innovation deutlich besser informiert als Unternehmer, die sich nicht für Förderprogramme bewerben. Hypothese 6 wird somit bestätigt. Dementsprechend wird Hypothese 7 (Unternehmer, die sich um Innovationsförderung bewerben, sind im Bereich Wirtschaftsförderung besser informiert als Unternehmer, die sich nicht für Förderprogramme bewerben. Aber sie sind nicht besser informiert, was den Bereich Innovation angeht.) aufgrund der empirischen Evidenz abgelehnt.

Die Ergebnisse der Befragung zeigen außerdem, dass keine Informationsasymmetrie in Bezug auf den Bewerbungsaufwand für das NIFP besteht, die in der Förderung unerfahrene Unternehmer von einer Bewerbung abhalten könnte. Tatsächlich unterschätzen sie den tatsächlichen Aufwand sogar in erheblichem Maße. Würden sie den eigentlichen Aufwand kennen, würde ihre Bewerbungsbereitschaft vermutlich weiter zurückgehen.

\subsubsection{Die Risikoeinstellung des Unternehmers}

Neben der Informiertheit der Unternehmer steht in vielen EntrepreneurshipTheorien die unternehmerische Risikoeinstellung im Mittelpunkt. So wird die relative Risikofreudigkeit der Unternehmer im Vergleich $\mathrm{zu}$ anderen Wirtschaftssubjekten diskutiert. In dieser Arbeit geht es jedoch darum, dass sich auch Unternehmer untereinander in ihrer Risikoeinstellung unterscheiden. In Abschnitt 5.5.5 wurde die Hypothese aufgestellt, dass sich innovative und nicht innovative Unternehmer in ihrer Risikoeinstellung nicht wesentlich unterscheiden (Hypothese 9). Im selben Abschnitt wurde die Argumentation erörtert, dass sich diejenigen innovativen Unternehmer, die eine starke Risikoaversion haben, für Innovationsfördermittel bewerben und den damit zusammenhängenden Be- 
werbungs- und Dokumentationsaufwand in Kauf nehmen, um ihr finanzielles Risiko zumindest teilweise abzusichern. Innovative Unternehmer mit einer eher geringen Risikoaversion bewerben sich dagegen nicht für Innovationsförderung, sondern übernehmen das Risiko selbst. Zusammenfassend wurde die Hypothese aufgestellt, dass Unternehmer, die sich um Innovationsförderung bewerben, risikoscheuer sind als Unternehmer, die sich nicht bewerben (Hypothese 10).

Um die beschriebenen beiden Hypothesen zu überprüfen, muss die Risikobereitschaft der Unternehmer gemessen werden. Zu diesem Zweck wurde eine Lotterie mit einem Sicherheitsäquivalent verwendet. Diese in der Literatur weit verbreitete Methode besitzt den Vorteil, dass sie - im Gegensatz zu einer Selbsteinschätzung der individuellen Risikoeinstellung - unabhängig von der Selbstwahrnehmung der Befragten ist. ${ }^{445}$ Zur Messung der Risikoaversion wurde folgende Fragestellung verwendet (Aufgabe 16 aus dem Fragebogen in Anhang $\mathrm{C}$ und D): ${ }^{446}$

Wie schätzen Sie persönlich die folgenden zwei Investitionsmöglichkeiten ein?

Möglichkeit A: Sie erhalten als Ertrag absolut sicher $100.000 €$.

Möglichkeit B: Sie erhalten als Ertrag mit einer Wahrscheinlichkeit von

$50 \%$ einen bestimmten Geldbetrag in Höhe von X.

Wie hoch müsste dieser Geldbetrag X sein, damit Sie beide Investitionsmöglichkeiten vollkommen gleich bewerten?

\section{$\square$ Kleiner als $190.000 € \quad \square$ Zwischen 210.000 und $250.000 €$ \\ $\square$ Zwischen 190.000 und $210.000 € \square$ Größer als $250.000 €$}

Die Zuordnung der Risikoeinstellungen zu den Antworten findet sich in Abbildung 30. Ein risikoneutraler Unternehmer würde sich für einen Betrag $X$ entscheiden, bei dem die Erwartungswerte der beiden Investitionsmöglichkeiten identisch sind:

$$
100.000 € \cdot 1=0 \cdot \frac{1}{2}+X \cdot \frac{1}{2}
$$

Somit entscheidet sich ein vollkommen risikoneutraler Unternehmer für einen Betrag X von $200.000 €=100.000 € \cdot 2$ und kreuzt die Kategorie „Zwischen 190.000 und $210.000 \epsilon^{\prime \prime}$ an. Einem risikoaversen Unternehmer reicht ein Betrag $X$ von $200.000 €$ nicht aus. Er verlangt eine Sicherheitsprämie, um die unsichere Investitionsmöglichkeit B ebenso zu bewerten wie die sichere Investitionsmög-

445 Vgl. Krahnen et al. (1997), S. 2 ff.

446 Die Fragestellung wurde in Anlehnung an Tversky und Kahneman (1986), S. S255, und Frage 121 des Personenfragebogens der Erhebung des Sozioökonomischen Panels von 2004, http://www.diw.de/deutsch/frageboegen/32045.html, zugegriffen am 17.06.2008, entwickelt. 
lichkeit A. Ein gering risikoaverser Unternehmer benötigt auch nur eine geringe Sicherheitsprämie, deswegen wird er die Größenkategorie ,zwischen 210.000 und $250.000 €$ “ ankreuzen, während ein stark risikoaverser Proband die Kategorie ,größer als $250.000 €$ “ ankreuzt, weil er eine höhere Sicherheitsprämie fordert. Dagegen verlangt ein risikofreudiger Proband nur einen Betrag X, der kleiner ist als der Betrag, den ein risikoneutraler Proband fordert. Deswegen werden diejenigen Probanden als risikofreudig identifiziert, die die Kategorie „kleiner als $190.000 \Theta^{\prime \prime}$ ankreuzen.

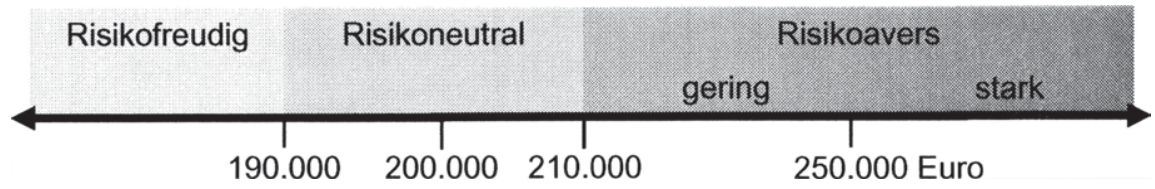

Abbildung 30: Einteilung in Risikoklassen (Eigene Darstellung).

Diese einfache Lotterie zur Bestimmung der Risikoaversion besitzt den Charme der Einfachheit, leidet aber gleichzeitig an einigen Unzulänglichkeiten. ${ }^{447}$ Erstens muss der Unternehmer auch hier - im Gegensatz zu den in Abschnitt 6.4 behandelten Fragen des Fragebogens - die Entscheidung zwingend selbst treffen. Deswegen werden in diesem Abschnitt nur die Fragebögen ausgewertet, bei denen der Unternehmer selbst den Fragebogen ausgefüllt hat (vgl. dazu Abschnitt 6.2). Zweitens könnte es der Fall sein, dass Unternehmer aus KMU unterschiedliche Antworten geben als Unternehmer aus Großunternehmen, da sie die Auszahlungen unterschiedlich bewerten. Da im Rahmen dieser Befragung allerdings $95,02 \%$ der befragten Unternehmen weniger als 250 Beschäftigte aufweisen und somit KMU darstellen, kann auch dieses Argument vernachlässigt werden.

Gewichtiger ist dagegen ein drittes Argument gegen die Messung der Risikoaversion mit Hilfe der beschriebenen einfachen Lotterie: Ein derartiges Spiel sollte direkt mit dem Probanden gespielt werden, um die Risikoaversion zuverlässig messen zu können, so dass die entsprechenden ,payoffs“ auch tatsächlich ausgezahlt werden. Solch ein Experiment konnte für die vorliegende Fragestellung allerdings nicht durchgeführt werden, da die beteiligten niedersächsischen Institutionen auf der Anonymität der zu befragenden geförderten Unternehmen bestanden. Daher ist das beschriebene Spiel das beste Instrument, um im Rahmen einer anonymen schriftlichen Befragung die unternehmerische Risikoaversion zu messen. Die Einfachheit des beschriebenen Spiels trägt außerdem dazu bei, dass der Fragebogen kurz gefasst werden kann und die Geduld der Probanden nicht überstrapaziert wird.

447 Vgl. Krahnen et al. (1997). 
Die Ergebnisse der Befragung weisen darauf hin, dass sich Unternehmer, die sich um Förderung bewerben, und Unternehmer, die sich nicht um Förderung bewerben, in ihrer Risikoeinstellung tatsächlich deutlich voneinander unterscheiden. Hypothese 10 wird also bestätigt: Unternehmer, die sich um Innovationsförderung bewerben, sind risikoscheuer als Unternehmer, die sich nicht bewerben. In Tabelle 11 sind die absoluten Häufigkeiten und in Abbildung 31 die relativen Häufigkeiten der verschiedenen Risikoeinstellungen abgebildet. Dabei beziehen sich die Häufigkeiten jeweils nur auf diejenigen, die die Risikoaufgabe tatsächlich beantwortet haben. Wie in Abbildung 31 dargestellt sind nur $4,46 \%$ der Unternehmer, die durch das NIFP gefördert wurden bzw. sich dafür beworben haben, risikofreudig (im Vergleich zu 10,81\% der nicht geförderten und 13,04\% der Unternehmer, die durch andere Förderprogramme gefördert wurden bzw. sich dafür beworben haben). ${ }^{448}$ In der anderen Extremgruppe der stark risikoaversen Unternehmer sind dagegen eindeutig mehr Unternehmer, die sich um Förderung beworben haben, $(36,61 \%$ der NIFP-Unternehmer und $34,78 \%$ der Unternehmer der anderen Programme) als Unternehmer, die sich nicht um Förderung beworben haben $(21,62 \%)$. Die bloße Häufigkeitsverteilung weist also schon darauf hin, dass Unternehmer, die sich um Förderung bewerben (und insbesondere die sich für das NIFP bewerbenden Unternehmer) risikoaverser sind als andere Unternehmergruppen.

Tabelle 11: Differenzierte Ergebnisübersicht der Risikoaversion nach Förderung

\begin{tabular}{lcccc}
\hline & $\begin{array}{c}\text { NIFP- } \\
\text { Förderung }\end{array}$ & $\begin{array}{c}\text { Andere } \\
\text { Förderung }\end{array}$ & $\begin{array}{c}\text { Keine } \\
\text { Förderung }\end{array}$ & Gesamt \\
\hline Risikofreudig & 5 & 3 & 8 & 16 \\
Risikoneutral & 31 & 7 & 24 & 62 \\
Gering risikoavers & 35 & 5 & 26 & 66 \\
Stark risikoavers & 41 & 8 & 16 & 65 \\
Keine Angabe & 149 & 3 & 20 & 33 \\
\hline Gesamt & 10 & 26 & 94 & 242 \\
\hline
\end{tabular}

Quelle: Eigene Auswertung.

448 Bei der Interpretation der Zahlen ist immer die geringe Fallzahl der Unternehmer, die sich für andere Förderprogramme beworben haben, zu bedenken, die leicht zu extremen Werten in der relativen Häufigkeitsverteilung führen kann.

449 Entweder wurde die Frage zur Risikoaversion nicht beantwortet oder der Fragebogen wurde nicht vom Unternehmer ausgefüllt. 


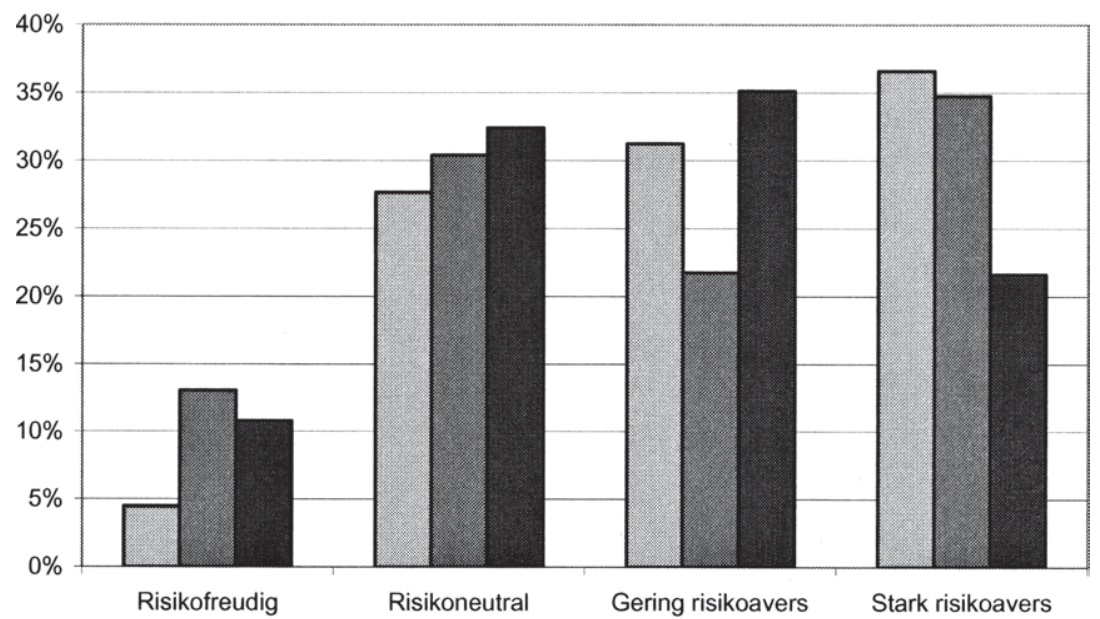

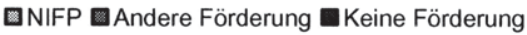

Abbildung 31: Risikoeinstellung nach Förderung (Eigene Darstellung).

Die Risikoeinstellung des Unternehmers ist also ein Unterscheidungsmerkmal von Unternehmern, die sich für Innovationsförderprogramme bewerben, und von Unternehmern, die sich nicht für solche Programme bewerben. Ob die beobachteten Differenzen statistisch signifikant sind, wird mit Hilfe des ChiQuadrat-Unabhängigkeitstests ermittelt. Allerdings können nicht alle drei nach Förderarten differenzierten Unternehmensgruppen miteinander verglichen werden, da die Gruppe der Unternehmen, die durch andere Förderprogramme gefördert wurden bzw. sich dafür beworben haben, mit insgesamt 24 Unternehmen eine relativ kleine Gruppe darstellt und die geringen Fallzahlen verzerrend wirken können. Voraussetzung zur Anwendung des Chi-Quadrat-Unabhängigkeitstests ist eine Fallzahl von mindestens fünf pro Zelle. Da diese Fallzahl für die beschriebene Gruppe nicht erfüllt ist, wird die Gruppe aus der Berechnung herausgenommen. Wenn nun die durch die NIFP-Unternehmer (gefördert oder beworben) mit den Unternehmern, die sich nicht um Förderung beworben haben, verglichen werden, ergibt sich ein signifikanter Unterschied auf dem $10 \%$ Niveau (vgl. Tabelle 13). Somit bestätigt das Ergebnis des Chi-Quadrat-Unabhängigkeitstests Hypothese 10: Unternehmer, die sich um Innovationsförderung bewerben, sind risikoscheuer als Unternehmer, die sich nicht bewerben.

Die Ergebnisse der Befragung weisen gleichzeitig darauf hin, dass sich die Risikoeinstellungen von innovativen und nicht innovativen Unternehmer deutlich voneinander unterscheiden und widersprechen somit Hypothese 9. In Tabelle 12 sind die absoluten und relativen Häufigkeiten und in Abbildung 32 die re- 
lativen Häufigkeiten bezogen auf diejenigen, die die Risikoaufgabe beantwortet haben, abgebildet. Nur 5,65\% der innovativen Unternehmer sind risikofreudig (im Vergleich zu 20,67\% der nicht innovativen Unternehmer).

Tabelle 12: Differenzierte Ergebnisübersicht der Risikoaversion nach Innovativität in absoluten und relativen Zahlen

\begin{tabular}{|c|c|c|c|c|}
\hline & $\begin{array}{l}\text { Innovative } \\
\text { Unternehmer }\end{array}$ & $\begin{array}{l}\text { Nicht innovative } \\
\text { Unternehmer }\end{array}$ & $\begin{array}{c}\text { Keine Angabe } \\
\text { zur Innovativität }\end{array}$ & Gesamt \\
\hline Risikofreudig & $10(5,29 \%)$ & $6(12,24 \%)$ & $(0 \%)$ & $16 \quad(6,61 \%)$ \\
\hline Risikoneutral & $54(28,57 \%)$ & $7(14,29 \%)$ & $1 \quad(25 \%)$ & $62(25,62 \%)$ \\
\hline $\begin{array}{l}\text { Gering risiko- } \\
\text { avers }\end{array}$ & $56(29,63 \%)$ & $8(16,33 \%)$ & $2 \quad(50 \%)$ & $66(27,27 \%)$ \\
\hline Stark risikoavers & $57(30,16 \%)$ & $8(16,33 \%)$ & $(0 \%)$ & $65(26,86 \%)$ \\
\hline Keine Angabe ${ }^{450}$ & $12(6,35 \%)$ & $20(40,82 \%)$ & $1 \quad(25 \%)$ & $33(13,64 \%)$ \\
\hline Gesamt & $189 \quad(100 \%)$ & $49 \quad(100 \%)$ & $4(100 \%)$ & $242 \quad(100 \%)$ \\
\hline
\end{tabular}

Quelle: Eigene Auswertung.

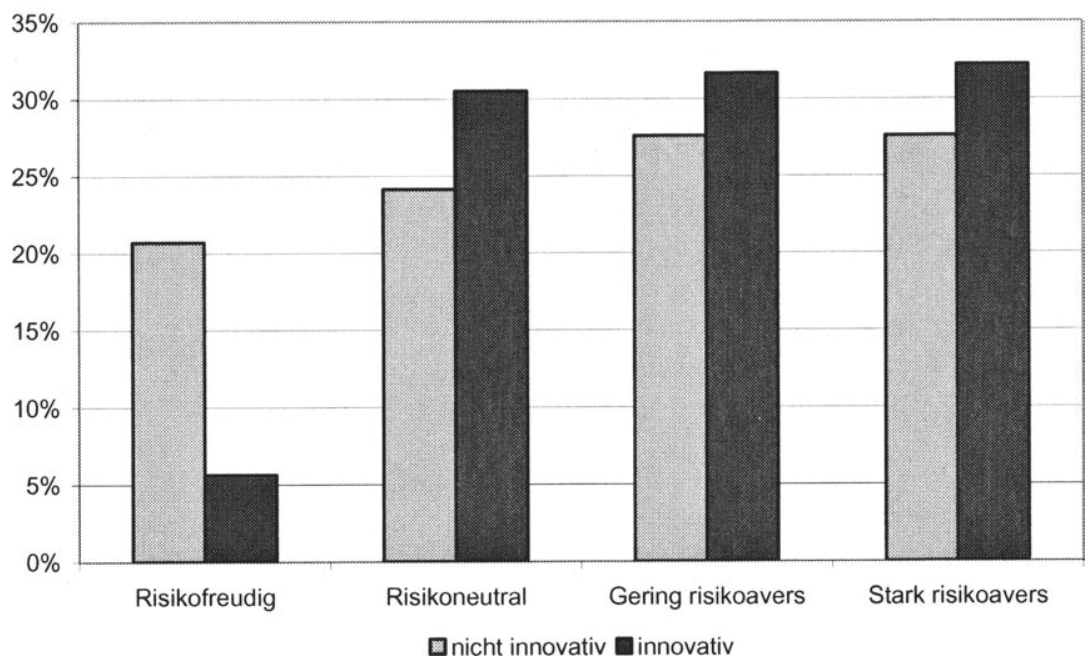

Abbildung 32: Risikoeinstellung nach Innovativität (Eigene Darstellung).

450 Entweder wurde die Frage zur Risikoaversion nicht beantwortet oder der Fragebogen wurde nicht vom Unternehmer ausgefüllt. 
Die bloße Häufigkeitsverteilung weist also schon darauf hin, dass innovative Unternehmer risikoaverser sind als nicht innovative Unternehmer. Beim Vergleich der beiden Unternehmergruppen mit Hilfe des Chi-Quadrat-Unabhängigkeitstests ergibt sich ein signifikanter Unterschied auf dem $5 \%$-Niveau (vgl. Tabelle 13). Somit widerlegt die empirische Evidenz Hypothese 9 und unterstützt die Argumentation von Drucker (1985) und Baron (2004) (vgl. Abschnitt 5.5.5): Innovative Unternehmer sind risikoscheuer als nicht innovative Unternehmer.

Tabelle 13: Ergebnisse des Chi-Quadrat-Unabhängigkeitstests

\begin{tabular}{lccc}
\hline & Prüfgröße $\chi^{2}$ & $\begin{array}{c}\text { Kritischer } \\
\text { Wert }^{451}\end{array}$ & $\operatorname{Pr}^{452}$ \\
\hline $\begin{array}{l}\text { Abweichungen zwischen durch das NIFP } \\
\text { geförderten und gar nicht geförderten }\end{array}$ & $6,38 *$ & 6,25 & $9,5 \%$ \\
$\begin{array}{l}\text { Unternehmen } \\
\begin{array}{l}\text { Abweichungen zwischen innovativen und } \\
\text { nicht innovativen Unternehmen }\end{array}\end{array}$ & $7,90 * *$ & 6,25 & $4,8 \%$ \\
\hline
\end{tabular}

Quelle: Eigene Auswertung.

Die Ergebnisse der Unternehmensbefragung weisen in der univariaten Analyse also darauf hin, dass Unternehmer, die sich für ein Förderprogramm bewerben, risikoscheuer sind als nicht geförderte Unternehmer. Es wird somit Hypothese 10 (Unternehmer, die sich um Innovationsförderung bewerben, sind risikoscheuer als Unternehmer, die sich nicht bewerben) bestätigt.

Es besteht des Weiteren ein signifikanter Unterschied in der Risikoeinstellung von innovativtätigen Unternehmern gegenüber nicht innovativ tätigen Unternehmern. Innovative Unternehmer sind risikoscheuer als nicht innovative Unternehmer. Somit wird Hypothese 9 (Innovative und nicht innovative Unternehmer unterscheiden sich nicht in ihrer Risikoeinstellung) nicht bestätigt. Es ist allerdings zu bedenken, dass der Vergleich von innovativen und nicht innovativen Unternehmern im Rahmen dieser Analyse nur darauf beruht, ob ein Unternehmen innovativ tätig ist oder nicht. Der Innovativitätsgrad wird hier noch nicht berücksichtigt.

Eine Erklärung für die beobachteten Unterschiede in der Risikoeinstellung zwischen innovativ tätigen und nicht innovativ tätigen Unternehmern liegt darin

451 Der kritische Wert bezieht sich auf ein Signifikanzniveau von $90 \%$.

452 Pr gibt die Wahrscheinlichkeit an, dass die Prüfgröße $\chi^{2}$ mindestens den berechneten Wert annimmt, unter der Annahme dass sich die Gruppen in ihrer Risikoeinstellung nicht voneinander unterscheiden. 
begründet, dass durch das NIFP geförderte Unternehmer in der Regel besonders innovative Projekte mit einem hohen Risiko durchführen. Deswegen wägen sie möglicherweise die Risiken jeglicher Investitionsprojekte genau ab und versuchen, vorhandene Risiken zu minimieren. Aus diesem Grund könnten geförderte Unternehmer auch mehr Wert auf eine hohe Informiertheit legen, um ihr Innovationsrisiko besser einschätzen zu können (vgl. Abschnitt 6.5.1).

In Abschnitt 5.5.5 wurde die Vermutung dargelegt, dass sich aus der Gruppe der innovativ tätigen Unternehmer vorwiegend die eher Risikoaversen bewerben, um die mit Innovationstätigkeit einhergehenden Technologie- und Marktrisiken zumindest teilweise finanziell abzusichern. Diese Vermutung kann hier nicht statistisch untersucht werden, weil die Fallzahlen hierfür zu gering sind. ${ }^{453}$ Der Zusammenhang zwischen Innovativität und Risikoeinstellung wird unter Einbeziehung weiterer Einflussfaktoren im Rahmen der multivariaten Analyse in Abschnitt 6.6 untersucht.

\subsubsection{Bewertung von Unternehmereigenschaften}

In Frage 15 des Fragebogens (vgl. Anhang $C$ und D) sollten die befragten Unternehmer sechs aufgeführte Unternehmereigenschaften auf einer Likert-Skala von 1 (= außerordentlich wichtig) bis 5 (= unwichtig) bewerten. Die genaue Formulierung der Frage lautete: „Welche der folgenden Eigenschaften schätzen Sie als wichtig ein, um ein erfolgreicher Unternehmer zu sein?" Allen Eigenschaften wurden relativ niedrige Werte zugewiesen. Somit wurden alle vorgegebenen Eigenschaften als sehr wichtig eingeschätzt. In der Bewertung der Eigenschaften unterscheiden sich die nach Förderung differenzierten Unternehmergruppen kaum voneinander. Interessant ist die Rangfolge der Eigenschaften, da sie einen Aufschluss darüber gibt, welche Unternehmereigenschaften in der Vorstellung der Unternehmer selbst einen erfolgreichen Unternehmer ausmachen (vgl. Tabelle 14). So wird die Risikobereitschaft am unwichtigsten bewertet. Eine mögliche Erklärung dafür besteht darin, dass Risikobereitschaft eine grundlegende Voraussetzung für die Existenzgründung bzw. die Übernahme eines bestehenden Unternehmens ist, aber im täglichen Geschäft des Unternehmers keine entscheidende Rolle spielt. Am wichtigsten werden die Entscheidungsfähigkeit und die gute Informiertheit des Unternehmers bewertet, so dass Cassons Sicht des Entrepreneurs als Informationsmanager (vgl. Abschnitt 5.4.3) von den Unternehmern selbst geteilt zu werden scheint. Die Schumpeter'sche Innovationsfähigkeit (vgl. Abschnitt 5.6.2), hier bezeichnet als „Aufgeschlossenheit gegenüber Neuem“, nimmt einen mittleren Rang ein. Es folgen Koordi-

453 Wie bereits im vorigen Abschnitt erwähnt, müssen für die Anwendung des $\chi^{2}$-Tests mindestens fünf Fälle pro Zelle vorliegen. Werden nur die innovativ tätigen Unternehmer als Grundgesamtheit berücksichtigt, so sind nur zwei Unternehmer risikoscheu, die sich in der Vergangenheit nicht für ein Förderprogramm beworben haben. 
nations- und Organisationsfähigkeit sowie Kreativität, die beide als weniger wichtig eingestuft werden.

Tabelle 14: Bewertung der Unternehmereigenschaften

\begin{tabular}{lccc}
\hline Unternehmereigenschaft & Mittelwert & Median & Rang \\
\hline Entscheidungsfähigkeit & 1,48 & 1 & 1 \\
Gut informiert sein & 1,55 & 1 & 2 \\
Aufgeschlossenheit gegenüber Neuem & 1,63 & 2 & 3 \\
Koordinations- und Organisationsfähigkeit & 1,83 & 2 & 4 \\
Kreativität & 2,06 & 2 & 5 \\
Risikobereitschaft & 2,33 & 2 & 6 \\
\hline
\end{tabular}

Quelle: Eigene Auswertung.

\subsubsection{Fazit}

Die univariaten Analysen ergeben, dass sich geförderte und nicht geförderte Unternehmer in Bezug auf bestimmte Eigenschaften und Fähigkeiten wie Risikoeinstellung und Informiertheit signifikant unterscheiden. Unternehmer, die sich in der Vergangenheit für ein Wirtschaftsförderprogramm beworben haben, sind deutlich besser informiert bezüglich der Bereiche Wirtschaftsförderung und Innovation, als Unternehmer, die sich nicht beworben haben. Unternehmer, die Förderung aus dem NIFP erhielten oder sich darum beworben haben, sind besonders gut informiert, was diese beiden Bereiche angeht. Hypothese 6 (Unternehmer, die sich um Innovationsförderung bewerben, sind in den Bereichen Innovation und Wirtschaftsförderung besser informiert als Unternehmer, die sich nicht für Förderprogramme bewerben) wird also bestätigt. Hypothese 7 (Unternehmer, die sich um Innovationsförderung bewerben, sind im Bereich Wirtschaftsförderung besser informiert als Unternehmer, die sich nicht für Förderprogramme bewerben. Aber sie sind nicht besser informiert, was den Bereich Innovation angeht) dagegen kann verworfen werden. Unternehmer, die sich für ein Förderprogramm bewerben, entsprechen also tatsächlich eher dem Casson'schen Entrepreneur und sind informierter bzw. besitzen eine höhere Aufmerksamkeit (,alertness") gegenüber für sie relevante Informationen.

Was die unternehmerische Risikoeinstellung angeht, so ergeben sich signifikante Unterschiede zwischen innovativen und nicht innovativen Unternehmern. Innovative Unternehmer sind risikoscheuer als nicht innovative Unternehmer. Dieses Ergebnis ist insofern interessant, als es in der Literatur widerstreitende Ansichten gibt (vgl. Abschnitt 5.5.5), weswegen in Hypothese 9 vermutet wurde, dass sich innovative und nicht innovative Unternehmer in ihrer Risikoeinstellung nicht unterscheiden. Für die wirtschaftspolitische Analyse von 
Innovationsförderung von besonderer Relevanz ist jedoch folgendes Ergebnis: Unternehmer, die sich für ein Förderprogramm bewerben, sind signifikant risikoscheuer als andere Unternehmer. Hypothese 10 (Unternehmer, die sich um Innovationsförderung bewerben, sind risikoscheuer als Unternehmer, die sich nicht bewerben) wird also durch die empirische Evidenz unterstützt.

Die beschriebenen Ergebnisse basieren bislang nur auf univariaten Analysen, d. h. sie stellen einen Vergleich zwischen den nach Förderung differenzierten Gruppen dar, ohne dass andere Einflussfaktoren berücksichtigt werden können. Die multivariate Analyse, in der Risikoeinstellung und Informiertheit im Zusammenhang mit anderen Einflussfaktoren untersucht wird, folgt im folgenden Abschnitt 6.6.

\subsection{Logit-Modell (logistische Regression)}

Im Folgenden wird das Logit-Modell verwendet, um zu untersuchen, welche unabhängigen metrischen und kategorialen Variablen in Zusammenhang damit stehen, ob ein Unternehmen Innovationsförderung erhält oder nicht. Somit lässt sich im Rahmen dieser multivariaten Analyse das Zusammenwirken der oben aufgeführten Unternehmereigenschaften, der Unternehmensstrukturdaten und dem Erhalt von Innovationsförderung erklären. ${ }^{454}$ Die binäre abhängige Variable y kann folgende Ausprägungen annehmen: entweder das Ereignis „Innovationsförderung aus dem NIFP erhalten“ $(y=1)$ oder das Ereignis „Keine Förderung erhalten bzw. darum beworben" $(y=0)$. Die Gruppe der durch andere Förderprogramme geförderten Unternehmen wurde aus der Analyse herausgenommen, weil keine Informationen über die Art der Förderung vorliegen und deswegen anzunehmen ist, dass diese Gruppe nicht sehr homogen ist. In dieser Gruppe können sich sowohl Unternehmen befinden, die Förderung für den Bereich F\&E/Innovation erhalten haben, als auch Unternehmen, die Förderung anderer Art erhalten haben, so dass die Unternehmen in dieser Gruppe sich möglicherweise stark voneinander unterscheiden. Ebenso wurden diejenigen Unternehmen, die sich zwar für das NIFP beworben, aber keine Förderung erhalten haben, aus der Grundgesamtheit herausgenommen, da sich beispielsweise die Einflussfaktoren der Innovativität nur auf diejenigen Unternehmen beziehen, die tatsächlich Fördermittel erhalten haben. Das Logit-Modell ist als Untersuchungsmethode besonders geeignet, weil es im Gegensatz zur linearen Regression die Variation der abhängigen Variable y auf das Intervall $[0,1]$ beschränkt.

Als unabhängige Variablen zur Abbildung der Unternehmensstruktur werden die metrischen Variablen Unternehmensalter und Beschäftigtenzahl sowie

454 Der beschriebene methodische Ansatz wird beispielsweise von Busom (2000) verwendet, um ein spanisches F\&E-Förderprogramm zu untersuchen. 
ein Dummy für die Existenz einer F\&E-Abteilung im Unternehmen verwendet. Zur Messung der Eigenschaft der Innovativität werden die in Abschnitt 6.4.3 beschriebenen Indikatoren verwendet, die jeweils metrische Variablen darstellen. Des Weiteren werden Dummies für die Kodierung der Branchenzugehörigkeit verwendet. ${ }^{455}$ Die erste Branchenkategorie steht dabei für den Bereich der Rohstoffherstellung und -verarbeitung. Die zweite Kategorie beinhaltet den Maschinen- und Anlagenbau sowie die Elektroindustrie. In der dritten Kategorie finden sich die restlichen Hochtechnologiebranchen, wie Medizintechnik, Optik und Fahrzeugbau. ${ }^{456}$ Die Referenzkategorie bildet die Gruppe der sonstigen Branchen des Verarbeitenden Gewerbes. Ein genauer Überblick über die Zuordnung der Branchendummies auf die verschiedenen Wirtschaftszweige der Industriebetriebe findet sich in Anhang K. Die Zuordnung der Branchendummies auf die entsprechenden Gewerke findet sich in Anhang L. Einen Überblick über die Verteilung der befragten Unternehmen nach Branchen gibt die folgende Tabelle 15.

Tabelle 15: Branchen der befragten Unternehmen

\begin{tabular}{lcc}
\hline Branche & $\begin{array}{c}\text { Prozentzahl der befragten } \\
\text { Unternehmen in der Branche }\end{array}$ & $\begin{array}{c}\text { Anzahl der befragten } \\
\text { Unternehmen in der Branche }\end{array}$ \\
\hline $\begin{array}{l}\text { Rohstoffherstellung und } \\
\text {-verarbeitung (Branche 1) }\end{array}$ & $19,25 \%$ & 46 \\
Maschinen- und Anlagenbau, & $28,03 \%$ & 67 \\
Elektroindustrie (Branche 2) & $11,72 \%$ & 28 \\
Hochtechnologie (Branche 3) & $41,00 \%$ & 98 \\
Sonstige Branchen (Branche 4) & $100,00 \%$ & 239 \\
\hline Gesamt & & \\
\hline
\end{tabular}

Quelle: Eigene Auswertung.

Die mit Hilfe der Entrepreneurship-Theorie beschriebenen und getesteten unternehmerischen Eigenschaften und Fähigkeiten stellen mögliche Einflussfaktoren des Erhalts von Innovationsförderung dar. Um diese Effekte zu quantifizieren, wird ein Logit-Modell berechnet, in dem diese Effekte als unabhängige Variablen eingeführt werden. Zur Messung der Eigenschaft der Informiertheit wird für jeden Unternehmer der Mittelwert seiner Bewertungen aller Informationsquellen

455 Zur Kodierung von kategorialen Variablen als Regressoren vgl. Tutz (2000), S. 19 f.

456 Eine ähnliche Zuordnung von Wirtschaftszweigen zum Hochtechnologiebereich findet sich in European Commission und Eurostat (2005), S. $102 \mathrm{f}$. Während dort die Zuordnung teilweise auf Basis der Dreisteller-Ebene der NACE erfolgt, wird die Zuordnung in dieser Arbeit aufgrund von ungenauen Angaben der Befragten generell auf Basis der Zweisteller-Ebene der NACE vorgenommen. 
gebildet, ein Indikator für die Uninformiertheit des Unternehmers (vgl. Abschnitt 6.5.1). Zur Kodierung der Eigenschaft der Risikoaversion (vgl. Abschnitt 6.5.2) werden Dummies für die Kategorien Risikofreudigkeit, geringe Risikoaversion und starke Risikoaversion gebildet. Die Referenzkategorie bildet die Gruppe der risikoneutralen Unternehmer. In der Berechnung des Logit-Modells werden - wie auch schon in den vorherigen Abschnitten - nur die Fragebögen betrachtet, die vom Unternehmer selbst ausgefüllt wurden.

Die in Tabelle 16 dargestellten Varianten des Logit-Modells unterscheiden sich in Bezug auf die verwendeten Innovativitätsindikatoren und in Bezug auf die Anzahl der hinein genommenen unabhängigen Variablen. In Modell 1 wurde die Innovationsintensität, in Modell 2 der Anteil der Beschäftigten im Bereich F\&E, in Modell 3 die Anzahl der Patente und in Modell 4 der Anteil der Marktneuheiten am Umsatz als Innovativitätsindikator verwendet. Zunächst wurde jeweils das Logit-Modell für alle 11 unabhängigen Variablen berechnet (Modellvariante a). Danach wurde das Logit-Modell jeweils noch einmal berechnet, wobei die beiden in Modellvariante a) nicht signifikanten Variablen Unternehmensalter und Beschäftigung aus der Analyse entfernt wurden, um zu überprüfen, ob sich die Resultate ohne diese beiden Variablen verändern (Modellvariante b), was nicht der Fall war.

Die Bedeutung der Koeffizienten des Logit-Modells erklärt sich über die Bildung der „odds ratio“, der Chancenverhältnisse. ${ }^{457}$ Unternehmen aus den Branchen Maschinen- und Anlagenbau/Elektroindustrie bzw. der Hochtechnologie haben eine deutlich größere Chance gefördert zu werden als Unternehmen aus der Referenzkategorie der sonstigen Unternehmen. So besteht eine ungefähr drei- bis fünffache Chance für Unternehmen aus der ersten Gruppe bzw. fünfbis achtfache Chance für die Unternehmen der zweiten Gruppe. Der Effekt einer eigenen F\&E-Abteilung ist dagegen nicht so eindeutig. In den Modellvarianten 1 (Innovationsintensität als Innovativitätsindikator), 2 (Beschäftigungsanteil im Bereich F\&E als Innovativitätsindikator) und 3 (Patentanzahl als Innovativitätsindikator ${ }^{458}$ ) haben Unternehmen, die eine F\&E-Abteilung besitzen, eine dreibis vierfache Chance Innovationsförderung zu erhalten, als Unternehmen ohne F\&E-Abteilung. Dagegen hat der Besitz einer eigenen F\&E-Abteilung in Modellvariante 4 (Anteil der Marktneuheiten am Umsatz als Innovativitätsindikator) keinen signifikanten Effekt. ${ }^{459}$

457 Vgl. Tutz (2000), S. 53; Long und Freese (2001), S. 132 ff.

458 Es werden die in Abschnitt 6.4.3 erwähnten Ausreißer aus der Analyse herausgenommen.

459 Dieses Ergebnis lässt sich evtl. durch die positiven Korrelationen zwischen den Innovativitätsindikatoren und der Existenz einer eigenen F\&E-Abteilung erklären. So liegt die punkt-biseriale Korrelation zwischen Existenz einer eigenen F\&E-Abteilung und Anteil der Marktneuheiten am Umsatz bei 0,36, während die Korrelationen zwischen Existenz einer eigenen F\&E-Abteilung und Innovationsintensität 0,19 bzw. zwischen eigener 
Tabelle 16: Ergebnisse des Logit-Modells

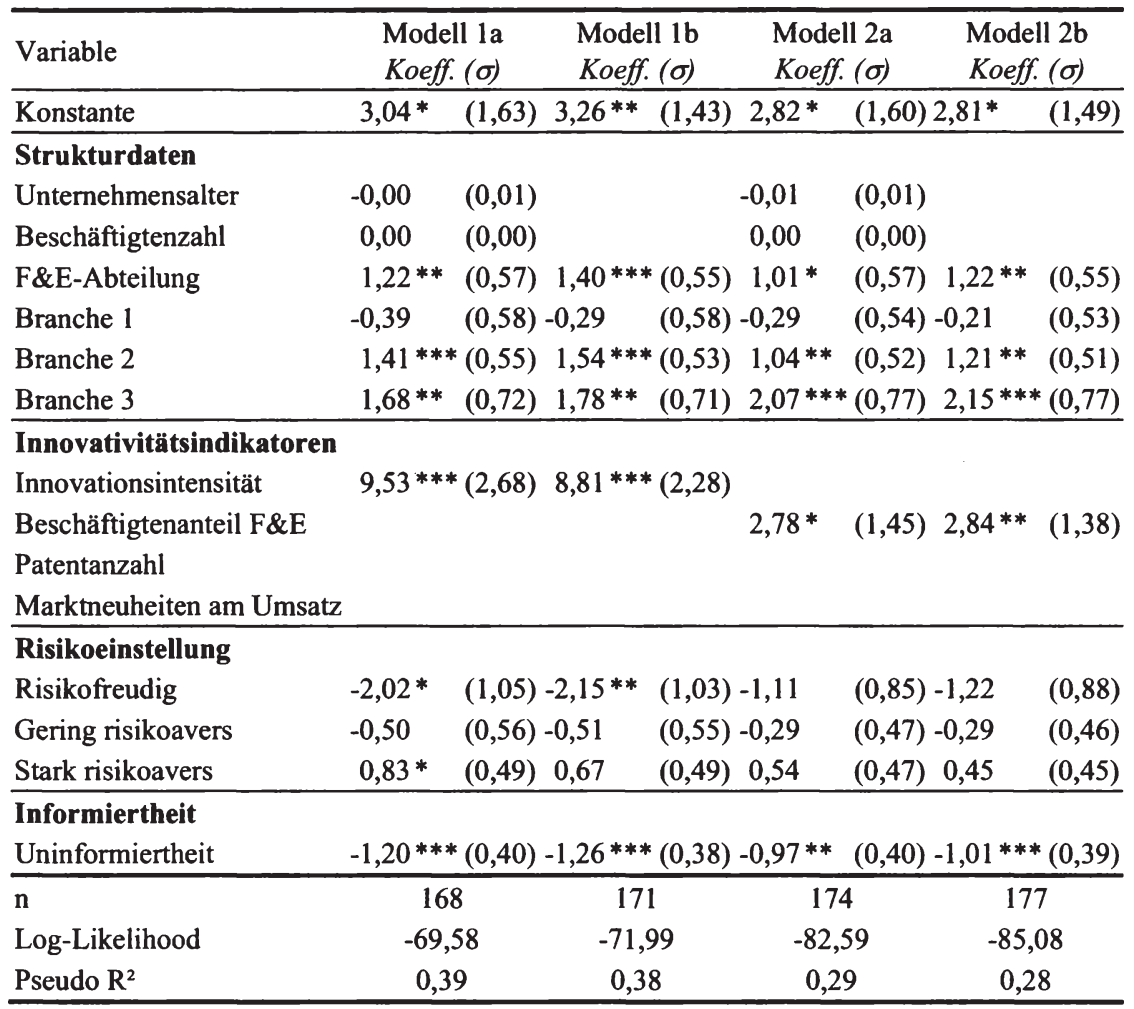

Huber-White Standardfehler in Klammern, ${ }^{*} 10 \%,{ }^{* *} 5 \%,{ }^{* * *} 1 \%$.

Quelle: Eigene Auswertung. ${ }^{460}$

F\&E-Abteilung und Beschäftigtenanteil im Bereich F\&E 0,17 betragen. Dennoch sind sowohl diese Korrelationen als auch die Korrelation zwischen Existenz einer eigenen F\&E-Abteilung und Beschäftigtenzahl $(0,26)$ und alle übrigen Korrelationen zwischen den im Modell verwendeten unabhängigen Variablen so gering, dass die Ergebnisse des Logit-Modells in jeder berechneten Variante aussagekräftig sind.

460 Die Ergebnisse verändern sich übrigens kaum, wenn die durch andere Förderprogramme geförderten Unternehmen in die Analyse mit einbezogen werden und generell geförderte Unternehmen mit nicht geförderten Unternehmen verglichen werden. In diesem Fall nimmt die binäre abhängige Variable y folgende Ausprägungen an: entweder ,Förderung aus einem beliebigen Programm erhalten“ $(y=1)$ oder „Keine Förderung erhalten oder darum beworben" $(\mathrm{y}=0)$. 
Tabelle 16: Ergebnisse des Logit-Modells (Fortsetzung)

\begin{tabular}{|c|c|c|c|c|c|c|c|c|}
\hline \multirow{2}{*}{$\begin{array}{l}\text { Variable } \\
\text { Konstante }\end{array}$} & \multicolumn{2}{|c|}{$\begin{array}{l}\text { Modell 3a } \\
\text { Koeff. }(\sigma)\end{array}$} & \multicolumn{2}{|c|}{$\begin{array}{l}\text { Modell 3b } \\
\text { Koeff. }(\sigma)\end{array}$} & \multicolumn{2}{|c|}{$\begin{array}{l}\text { Modell 4a } \\
\text { Koeff. }(\sigma)\end{array}$} & \multicolumn{2}{|c|}{$\begin{array}{l}\text { Modell 4b } \\
\text { Koeff. }(\sigma)\end{array}$} \\
\hline & $3,84 * *$ & $(1,34)$ & $3,48 * * *$ & * $(1,28)$ & $2,81 *$ & $(1,56)$ & $2,93 * *$ & $(1,45)$ \\
\hline \multicolumn{9}{|l|}{ Strukturdaten } \\
\hline Unternehmensalter & $-0,01$ & $(0,01)$ & & & $-0,01$ & $(0,01)$ & & \\
\hline Beschäftigtenzahl & $-0,00$ & $(0,00)$ & & & 0,00 & $(0,00)$ & & \\
\hline F\&E-Abteilung & $1,15 * *$ & $(0,57)$ & $1,30 * *$ & $(0,56)$ & 0,61 & $(0,56)$ & 0,88 & $(0,54)$ \\
\hline Branche 1 & $-0,78$ & $(0,53)$ & $-0,98 * *$ & $(0,50)$ & $-0,49$ & $(0,56)$ & $-0,39$ & $(0,55)$ \\
\hline Branche 2 & 0,40 & $(0,48)$ & 0,46 & $(0,45)$ & $0,92 *$ & $(0,49)$ & $1,13^{* *}$ & $(0,49)$ \\
\hline Branche 3 & 1,73 & $(1,14)$ & $2,09 *$ & $(1,13)$ & $1,52 * *$ & $(0,72)$ & $1,59 * *$ & $(0,72)$ \\
\hline
\end{tabular}

\section{Innovativitätsindikatoren}

Innovationsintensität

Beschäftigtenanteil F\&E

Patentanzahl $0,24^{*}(0,13) 0,21^{*} \quad(0,12)$

Marktneuheiten am Umsatz $4,05 * * *(1,24) 3,86 * * *(1,15)$

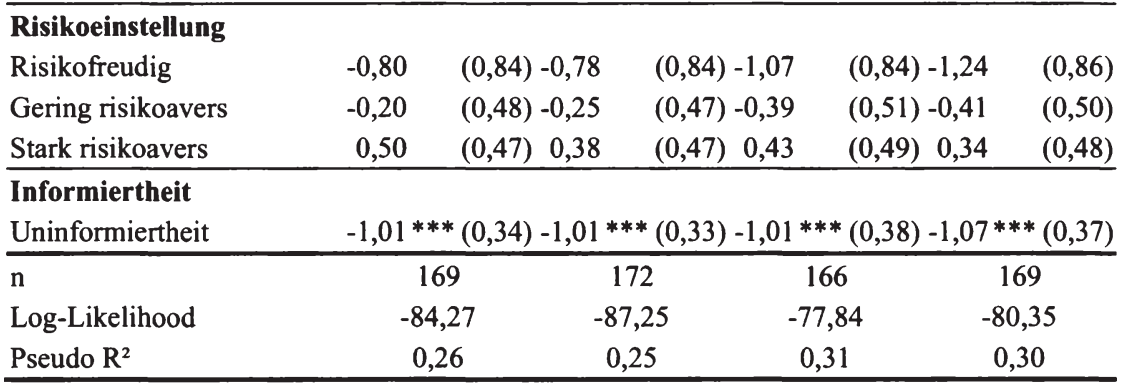

Ebenfalls sehr aussagekräftig sind die Ergebnisse des Logit-Modells für die Informiertheit des Unternehmers. Bei Konstanthaltung der übrigen Einflussfaktoren haben informiertere Unternehmer eine höhere Chance, Innovationsförderung zu erhalten. Eine Senkung des Uninformiertheitsindikators um einen Punkt führt zu einer Erhöhung der Chance, Innovationsförderung zu erhalten, um das 2,5bis 3,3-fache, je nach Modellvariante. Hypothese 6 (Unternehmer, die sich um Innovationsförderung bewerben, sind in den Bereichen Innovation und Wirtschaftsförderung besser informiert als Unternehmer, die sich nicht für Förderprogramme bewerben) wird hier noch einmal bestätigt.

Risikofreudige Unternehmer haben bei Konstanthaltung aller übrigen Einflussfaktoren nur eine 0,1- bis 0,3-fache Chance im Vergleich zu risikoneutralen Unternehmern (Referenzkategorie), Innovationsförderung zu erhalten. Im Umkehrschluss haben risikoneutrale Unternehmer eine drei- bis zehnfache Chance des Erhalts von Innovationsförderung im Vergleich zu risikofreudigen Unter- 
nehmern. Die multivariate Analyse bestätigt also zunächst die Ergebnisse aus Abschnitt 6.5.2 in Bezug auf Risikofreudigkeit: Risikofreudige Unternehmer erhalten deutlich seltener Innovationsfördermittel als risikoneutrale Unternehmer. Dieses Ergebnis ist allerdings nur in Modellvariante $1 \mathrm{~b}$ ) auf dem $5 \%$ Niveau und in Modellvariante 1a) auf dem $10 \%$-Niveau signifikant. In den anderen drei Modellvarianten wird das Signifikanzniveau von $10 \%$ mehr oder weniger knapp verfehlt. Der positive Koeffizient von stark risikoaversen Unternehmern weist darauf hin, dass diese im Vergleich zu risikoneutralen Unternehmern eher Förderung erhalten, was Hypothese 10 (Unternehmer, die sich um Innovationsförderung bewerben, sind risikoscheuer als Unternehmer, die sich nicht bewerben) bestätigt. Dieses Ergebnis ist allerdings nur in Modellvariante 1a) auf dem $10 \%$-Niveau signifikant. In dieser Spezifikation haben stark risikoaverse Unternehmer eine 2,3-fache Chance im Vergleich zur Referenzgruppe der risikoneutralen Unternehmer, Innovationsförderung zu erhalten. Die Risikokategorie ,gering risikoavers“ hat dagegen in keiner Modellvariante einen signifikanten Einfluss.

Besonders stark ausgeprägt ist der Effekt der Innovativität auf die Förderung. Wenn ein Unternehmen seine Innovationsintensität um $1 \%$ steigert, dann steigt seine Chance, Innovationsförderung zu erhalten, um das 60-fache (Modellvariante 1b). Der hohe Koeffizient erklärt sich durch das Vorliegen von Simultanität. Denn erhaltene Innovationsfördermittel zählen in einem Unternehmen zu den gesamten aufgeführten Innovationsaufwendungen eines Jahres. Der Erhalt von Innovationsförderung beeinflusst somit für die im Jahr 2006 geförderten Unternehmen die Höhe der Innovationsintensität direkt positiv, so dass der Koeffizient der Innovationsintensität im Logit-Modell überschätzt wird. Mit Hilfe der vorliegenden Daten ist die Simultanität leider nicht vollständig auszuräumen. Jedoch unterliegen die anderen Innovativitätsindikatoren dem Simultanitätsproblem in deutlich geringerem Maße. So wird beispielsweise der Beschäftigungsanteil im Bereich F\&E eher weniger durch den Erhalt von Innovationsfördermitteln beeinflusst, sondern stellt eher eine mittelfristig bis langfristig konstante Größe dar. ${ }^{461}$ Die Patentanzahl und der Anteil der Marktneuheiten am Umsatz könnten zwar indirekt durch den Erhalt von Fördermitteln und die dadurch erst ermöglichte Innovationstätigkeit beeinflusst werden. Jedoch besteht eine Zeitverzögerung zwischen der Entwicklung der Innovation, der damit eventuell verbundenen Patentierung und der Durchsetzung der Innovation am Markt. Die in dieser Arbeit betrachteten Unternehmen haben ihre Förderung im Zeitraum von 2004-2007 erhalten, so dass die Outputeffekte der Fördermittel überschaubar erscheinen. Deswegen kann das Simultanitätsproblem im Folgenden vernachlässigt werden.

461 Vgl. Busom (2000), S. 128. 
Für die berechneten Logit-Modellvarianten unter Einbezug der anderen aufgeführten Innovativitätsindikatoren ergeben sich ähnliche Resultate bzgl. der Richtung der Koeffizienten und ihrer Signifikanz, nur haben die positiven Effekte ein deutlich geringeres Ausmaß. Steigt der Beschäftigtenanteil im Bereich F\&E um $10 \%$, dann steigt die Chance des Unternehmens ungefähr um das 1,7fache (Modellvariante 2b). Steigt die Anzahl der Patente des Unternehmens um 1, so steigt die Chance, Innovationsfördermittel zu erhalten, um das 1,2-fache (Modellvariante 3b). Steigt der Anteil der Marktneuheiten am Umsatz um $10 \%$, dann steigt die Chance des Unternehmens um das 4,7-fache (Modellvariante 4b). Hypothese 5 (Geförderte Unternehmen weisen eine höhere Innovationstätigkeit auf als nicht geförderte Unternehmen) wird somit auch durch die multivariate Analyse bestätigt.

Innovationsförderung kann sowohl dazu dienen, bislang wenig innovative Unternehmen zu fördern (allokationspolitische Begründung), als auch schon innovative Unternehmen in ihren Bemühungen zu unterstützen (wachstumspolitische Begründung) (vgl. Kapitel 2). Wird davon ausgegangen, dass Innovationsförderung nur für überhaupt innovative Unternehmen in Frage kommt, sollte die Grundgesamtheit für die Berechnung des Logit-Modells auf die überhaupt innovativ tätigen Unternehmen eingegrenzt werden. Bei dieser Betrachtung ergeben sich ähnliche Resultate in Bezug auf die Richtung der Koeffizienten für die Variablen Unternehmensalter, Beschäftigtenzahl, der Branchendummies, der Innovativitätsindikatoren sowie Uninformiertheit (vgl. Tabelle 19 in Anhang M). Die Höhe der Koeffizienten sowie die Signifikanz sind im Schnitt geringer als in der Analyse der Gesamtheit. Stark risikoaverse Unternehmer haben ebenfalls wie in der Gesamtbetrachtung eine deutlich höhere Chance, gefördert zu werden als die Vergleichsgruppe der risikoneutralen Unternehmer. Dieses Ergebnis ist jedoch nur in Modellvariante 1a) auf dem $10 \%$-Niveau signifikant. Bei zwei Erklärungsvariablen ergeben sich jedoch in der reduzierten Betrachtung deutliche Unterschiede. Erstens ist die Existenz einer eigenen F\&E-Abteilung nur in der Modellvariante $1 \mathrm{~b}$ ) ein signifikantes Unterscheidungsmerkmal von geförderten und nicht geförderten Unternehmen. Im Gegensatz zur Gesamtbetrachtung gibt der Besitz einer eigenen F\&E-Abteilung bei ausschließlicher Betrachtung von innovativen Unternehmen also keinen Hinweis auf eine höhere Chance, Fördermittel zu erhalten. Die Innovativitätsindikatoren Innovationsintensität und Anteil der Marktneuheiten am Umsatz stellen hier deutlich sinnvollere Indikatoren zur Abgrenzung dar. Zweitens ergibt sich ein Unterschied bei Betrachtung der risikofreudigen Unternehmer. Ihr Koeffizient ist nur in zwei von acht Modellvarianten negativ und in keiner Modellvariante signifikant. Dieses Ergebnis deutet darauf hin, dass sich aus der Gruppe der innovativ tätigen Unternehmer nicht die eher Risikoaversen bewerben, wie eine Vermutung in Abschnitt 5.5.5 besagte. Es muss allerdings berücksichtigt werden, dass die vorgenommenen Einschränkungen zu einer Fallzahl von nur noch 124 bis 149 betrachteten Unternehmen füh- 
ren. Das sinkende Pseudo $\mathrm{R}^{2}$ gibt außerdem einen Hinweis darauf, dass die auf innovativ tätige Unternehmen beschränkten Logit-Modellvarianten einen geringeren Erklärungsgehalt besitzen als die oben aufgeführten Logit-Modellvarianten.

Die Ergebnisse der univariaten statistischen Analysen in den vorangegangenen Abschnitten deuteten bereits darauf hin, dass sich geförderte und nicht geförderte Unternehmen in Bezug auf bestimmte Unternehmens- und Unternehmercharakteristika deutlich voneinander unterscheiden. Die Ergebnisse des Logit-Modells unterstützen diese Feststellung nunmehr unter Verwendung eines multivariaten statistischen Verfahrens. Es ist somit festzustellen, dass nicht nur die Eigenschaften des Unternehmens, wie Innovativität und Branchenzugehörigkeit, sondern auch die Fähigkeiten und Eigenschaften des Unternehmers, wie seine Informiertheit und auch seine Risikoeinstellung, einen wichtigen Erklärungsbeitrag bei der Unterscheidung von geförderten und nicht geförderten Unternehmen leisten können. Die Beschäftigtenzahl und das Unternehmensalter haben in der multivariaten Analyse im Gegensatz zur univariaten Analyse jedoch keinerlei Erklärungsgehalt für die Differenzierung zwischen geförderten und nicht geförderten Unternehmen.

\subsection{Zusammenfassung der Ergebnisse und Beantwortung der Hypothesen}

In diesem Kapitel wurde die Fragestellung erörtert, ob sich geförderte und nicht geförderte Unternehmen in Bezug auf generelle Charakteristika des Unternehmens sowie Eigenschaften und Fähigkeiten der verantwortlichen Unternehmer unterscheiden. Hierzu wurden drei Gruppen betrachtet: Unternehmen, die Fördermittel aus dem NIFP erhalten haben bzw. sich um solche beworben haben, Unternehmen, die Fördermittel aus anderen Programmen erhalten bzw. sich darum beworben haben, und Unternehmen, die sich weder um Förderung beworben noch Fördermittel erhalten haben. Wenn solche systematischen Unterschiede zwischen den Unternehmen existieren, deutet dies darauf hin, dass Innovationsförderprogramme nur von bestimmten Unternehmen bzw. Unternehmern wahrgenommen und für interessant befunden werden. Nur diese bewerben sich überhaupt um Fördermittel. Um diese Fragestellung zu untersuchen, wurden die Ergebnisse einer im Mai/Juni 2007 durchgeführten Unternehmensbefragung von im Rahmen des NIFP geförderten Unternehmen sowie einer Vergleichsgruppe untersucht und anhand der im theoretischen Teil dieser Arbeit formulierten Hypothesen diskutiert.

Unternehmen, die sich erfolgreich oder erfolglos um Förderung beworben haben, unterscheiden sich in Bezug auf Unternehmensgröße (gemessen mittels der Beschäftigtenanzahl) und Unternehmensalter nur in der univariaten Analyse 
signifikant von Unternehmen, die sich nicht um Förderung beworben haben. Die multivariate Analyse zeigt dagegen, dass Unternehmensgröße und -alter keinen Erklärungsbeitrag zur Differenzierung zwischen geförderten und nicht geförderten Unternehmen liefert.

\title{
Hypothese 5: Geförderte Unternehmen weisen eine höhere Innovati- onstätigkeit auf als nicht geförderte Unternehmen. (bestätigt)
}

Die durch das NIFP geförderten Unternehmer entsprechen eher dem Schumpeter'schen Entrepreneur und sind tatsächlich innovativer als durch andere Wirtschaftsprogramme geförderte und nicht geförderte Unternehmen, unabhängig vom verwendeten Innovativitätsindikator (verwendete Indikatoren: Innovationsintensität, Anteil der Beschäftigten im Bereich F\&E, Patente, Anteil der Marktneuheiten am Umsatz). Dieses Ergebnis bestätigt sich in uni- und multivariaten Analysen.

\begin{abstract}
Hypothese 6: Unternehmer, die sich um Innovationsförderung bewerben, sind in den Bereichen Innovation und Wirtschaftsförderung besser informiert als Unternehmer, die sich nicht für Förderprogramme bewerben. (bestätigt)
\end{abstract}

Hypothese 7: Unternehmer, die sich um Innovationsförderung bewerben, sind im Bereich Wirtschaftsförderung besser informiert als Unternehmer, die sich nicht für Förderprogramme bewerben. Aber sie sind nicht besser informiert, was den Bereich Innovation angeht. (nicht bestätigt)

Bei Betrachtung der Eigenschaften und Fähigkeiten der befragten Unternehmer im Rahmen der Entrepreneurship-Theorie ergeben sich ebenfalls deutliche Unterschiede zwischen den Gruppen. Unternehmer, die sich für ein Innovationsförderprogramm bewerben, nutzen fast alle in der Befragung angegebenen Informationsquellen in den Bereichen Wirtschaftsförderung und Innovation intensiver als nicht geförderte Unternehmer. Es wurde ein Informiertheitsindikator gebildet, dessen Betrachtung ergab, dass Unternehmer, die sich für ein Förderprogramm bewerben, bezogen auf die genannten Bereiche besser informiert sind. Dieses Ergebnis bestätigte sich in uni- und multivariaten Analysen. Unternehmer, die sich für ein Innovationsförderprogramm bewerben, entsprechen also stärker dem Casson'schen Entrepreneur als Unternehmer, die sich nicht für Wirtschaftsförderprogramme bewerben. Informiertheit stellt also de facto ein Selektionskriterium bei einer Bewerbung für ein Innovationsförderprogramm dar. Inwiefern dies wirtschaftspolitisch gewollt sein könnte, wird in den wirtschaftspolitischen Implikationen dieser Arbeit im folgendem Kapitel diskutiert. 
Hypothese 8: Der Bewerbungs- und Dokumentationsaufwand ist in der Realität nicht so groß wie in der Vorstellung derjenigen Unternehmer, die sich nicht für das NIFP beworben haben. (nicht bestätigt)

Beim Vergleich des tatsächlich geleisteten Bewerbungsaufwands der Unternehmer, die sich für das NIFP beworben haben, mit der Vorstellung der nicht geförderten Unternehmer vom zu leistenden Bewerbungsaufwand ergeben sich eklatante Unterschiede. Nicht geförderte Unternehmer halten die Antragstellung zwar generell eher für aufwendig als geförderte Unternehmer. Doch unterschätzen sie den mit der Antragstellung verbundenen Arbeitsaufwand erheblich.

\section{Hypothese 9: Innovative und nicht innovative Unternehmer unter- scheiden sich nicht in ihrer Risikoeinstellung. (nicht bestätigt)}

Hypothese 10: Unternehmer, die sich um Innovationsförderung bewerben, sind risikoscheuer als Unternehmer, die sich nicht bewerben. (teilweise bestätigt)

Die Ergebnisse der Befragung weisen darauf hin, dass Unternehmer, die sich für ein Förderprogramm wie das NIFP bewerben, risikoscheuer sind als nicht geförderte Unternehmer. Für diese empirische Evidenz gibt es verschiedene sinnvolle Erklärungsansätze. Eine mögliche Erklärung besteht darin, dass sich vorwiegend die risikoscheueren Unternehmer bewerben und den mit einer Bewerbung einhergehenden erheblichen Bewerbungs- und Dokumentationsaufwand deswegen in Kauf nehmen, weil sie die eventuell erheblichen Innovationsrisiken wenigstens teilweise finanziell absichern wollen. Volkswirtschaftlich gesehen ist eine Förderung dieser Unternehmer sinnvoll, weil diese ohne Fördermittel ihre Innovationsprojekte mit angenommenen positiven Spillover-Effekten für die Volkswirtschaft nicht durchgeführt hätten. Dieses Ergebnis konnte in der multivariaten Analyse allerdings nicht bestätigt werden.

Eine alternative Erklärung für die beobachteten Unterschiede in der Risikoeinstellung besteht darin, dass die durch das NIFP geförderten Unternehmer in der Regel sehr innovative Projekte mit einem hohen Risiko bearbeiten, weswegen sie generell Investitionsprojekte und deren Risiken genauer abwägen und versuchen, die vorhandenen Risiken zu minimieren. Aus diesem Grund könnten sie auch daran interessiert sein, sich gut zu informieren, um ihr Innovationsrisiko besser einschätzen zu können. Dieser Erklärungsansatz wird dadurch bestätigt, dass innovative Unternehmer risikoscheuer sind als nicht innovativ tätige Unternehmer.

Zusammengefasst können neben der Branchenzugehörigkeit sowohl die Innovativität des Unternehmens als auch die Risikoeinstellung und die Informiertheit der Unternehmer einen wichtigen Erklärungsbeitrag für die Beantwortung 
der Fragestellung leisten, inwiefern sich geförderte und nicht geförderte Unternehmen unterscheiden. Die Beschäftigtenzahl und das Unternehmensalter liefern dagegen keinen Erklärungsbeitrag bei der Differenzierung zwischen geförderten und nicht geförderten Unternehmen.

Die aufgeführten Unterschiede zwischen geförderten und nicht geförderten Unternehmen bzw. Unternehmern sind für die Wirtschaftspolitik von erheblicher Bedeutung, da sie eine Erklärung dafür liefern, welche Unternehmer sich überhaupt für Innovationsförderprogramme bewerben und Fördermittel erhalten. Bei der Beurteilung der Förderwürdigkeit eines Unternehmens durch verschiedene Bürokratieebenen steht zwar das konkrete Innovationsprojekt im Vordergrund und nicht die hier untersuchten grundlegenden Charakteristika von Unternehmen und Unternehmern. Doch deuten die bestehenden systematischen Unterschiede zwischen geförderten und nicht geförderten Unternehmen darauf hin, dass Innovationsförderprogramme hauptsächlich von einer bestimmten Unternehmens- bzw. Unternehmergruppe wahrgenommen und für interessant befunden werden, um sich für die dazugehörigen Fördermittel zu bewerben: den innovativen Unternehmen aus bestimmten Branchen (Maschinen- und Anlagenbau, Elektrotechnik sowie Hochtechnologie), die von einem Unternehmer geleitet werden, der eher risikoscheu ist und der Wert auf eine umfassende Meinungsbildung durch die Nutzung von verschiedenen Informationsquellen legt, um sich über die Bereiche Wirtschaftsförderung und Innovation zu informieren.

Unter Berücksichtigung all dieser Erkenntnisse können nun die ersten vier im Rahmen dieser Arbeit aufgestellten Hypothesen überprüft werden, die sich auf die mit der Förderung verbundenen Interessen von Politik und Bürokratie beziehen.

Hypothese 1: Es werden eher risikoreiche Innovationsprojekte von risikofreudigen Unternehmern, die in Bezug auf Innovation wenig Erfahrung haben, gefördert (allokationspolitische Begründung). (nicht bestätigt)

Hypothese 2: Es werden eher sichere Innovationsprojekte risikoscheuer Unternehmer, die über ausreichend Erfahrung die Innovationstätigkeit betreffend verfügen, gefördert (wachstumspolitische Begründung). (teilweise bestätigt)

Hypothese 3: Politiker haben den Anreiz, relativ sichere Innovationsprojekte risikoscheuer Unternehmer zu fördern, die erfahren in der Innovationstätigkeit sind, was genau der Zielgruppe der wachstumspolitischen Begründung für Innovationsförderung entspricht. (teilweise bestätigt) 
Hypothese 4: Die verschiedenen Gruppen der Bürokratie haben den Anreiz, eher sichere Innovationsprojekte risikoscheuer Unternehmer zu fördern, die über ausreichend Erfahrung die Innovationstätigkeit betreffend verfügen. (teilweise bestätigt)

Die Befragung lieferte keine Informationen über die einzelnen geförderten Innovationsprojekte und das mit ihnen verbundene Innovationsrisiko. Deswegen kann dieser Teil der Hypothesen nicht überprüft werden. Es fand sich jedoch empirische Evidenz dafür, dass eher risikoscheue Unternehmer, die erfahren in der Innovationstätigkeit sind, gefördert wurden. Die empirische Evidenz unterstützt somit die durch die Formulierung der Förderrichtlinie gestützte Hypothese, dass das NIFP ein wachstumspolitisch begründetes Förderprogramm darstellt und in der Praxis auch so umgesetzt wird. Die innovationspolitische Zielgruppe der verantwortlichen Politiker wird von den mit der Durchführung des Förderprogramms betrauten Bürokraten ausgewählt, indem eher in der Innovationstätigkeit erfahrene risikoscheue Unternehmer gefördert werden. 


\section{Zusammenfassung und Ausblick}

\subsection{Zusammenfassung der Ergebnisse}

Im Folgenden werden die Ergebnisse dieser Arbeit noch einmal kurz zusammengefasst:

- Die bis heute verwendete maßgebliche Definition des Innovationsbegriffs geht auf Schumpeter zurück, für dessen Innovationsbegriff zwei Kriterien erfüllt sein müssen: Die qualitative Neuartigkeit eines Produktes bzw. Verfahrens (Invention) sowie deren Durchsetzung am Markt oder innerhalb einer Organisation. (Abschnitt 2.1)

- Ausgangspunkt dieser Arbeit sind die Feststellungen, dass Innovativität und Wirtschaftswachstum positiv zusammenhängen und dass durch Marktversagen nicht ausreichend Innovation in der Gesellschaft hergestellt werden. Aus diesem Grund zählt die Europäische Kommission den Bereich Innovation bzw. F\&E zu den Schwerpunktbereichen staatlicher Beihilfen. ${ }^{462}$ So erhalten innovative Unternehmen des Verarbeitenden Gewerbes in Deutschland zu einem großen Teil staatliche Unterstützung, wobei das Instrument der Innovationsförderung eine wesentliche Rolle spielt. (Abschnitt 1.1)

- Das Vorliegen von positiven externen Effekten im Bereich F\&E, das hohe Risiko von Innovationsprojekten und die damit zusammenhängende Investitionszurückhaltung sowie Finanzierungsschwierigkeiten der Unternehmer führen zu einem allokativen Marktversagen und begründen ordnungsökonomisch ein staatliches Eingreifen in den Marktmechanismus mittels Innovationsförderung. Ein weiteres Argument für diese Form der staatlichen Intervention stellt der positive Zusammenhang zwischen technischem Fortschritt und Wirtschaftswachstum nach den Erkenntnissen der endogenen Wachstumstheorie dar. Hieraus lassen sich zwei mögliche Zielgruppen von Innovationsförderung ableiten: Aus allokationspolitischen Gründen sollten eher risikoreiche Innovationsprojekte von risikofreudigen Unternehmern, die in Bezug auf Innovation wenig Erfahrung haben, gefördert werden. Aus wachstumspolitischen Erwägungen heraus sollten eher sichere Innovationsprojekte risikoscheuer Unternehmer gefördert werden, die ausreichend erfahren in der Innovationstätigkeit sind. (Abschnitte 2.3 und 2.4)

462 Vgl. Kommission der Europäischen Gemeinschaften (2005), Teil II, Rn. 25. 
- Bei der herkömmlichen Messung der Fördereffekte im Sinne des Einflusses auf volkswirtschaftliche Schlüsselgrößen tritt eine Schwierigkeit auf: Die $\mathrm{Zu}$ ordnung von Unternehmen zu den beiden Gruppen erfolgt nicht zufällig. Bestimmte Faktoren sind dafür verantwortlich, dass sich Unternehmen um Förderung bewerben, und beeinflussen gleichzeitig die weitere Unternehmensentwicklung. Die Trennung dieser beiden Effekte ist bislang noch wenig erforscht. Daraus ergibt sich die Fragestellung, inwiefern sich Unternehmen, die Fördermittel aus einem Innovationsförderprogramm erhalten bzw. sich darum beworben haben, von Unternehmen, die sich nicht für Fördermittel bewerben und dementsprechend auch keine Förderung erhalten, unterscheiden. (Abschnitt 4.5)

- Ein Innovationsförderprogramm stellt eine Institution dar, da es aus einer Gesamtheit von Regeln zur Auswahl der geförderten Unternehmen und zur Durchführung der Fördermaßnahmen besteht. Diese Regeln beeinflussen das Verhalten der beteiligten Individuen aus den Akteursgruppen Politiker, Bürokraten und Unternehmer. Institutionelle Durchsetzungsmechanismen bestehen in der Auswahl der zu fördernden Unternehmen und der Überprüfung der Fördermittelverwendung. (Abschnitt 3.1)

- Die Analyse der Institution Innovationsförderung unter Anwendung der Theorie der Neuen Institutionenökonomik kommt zu dem Ergebnis, dass regionale Politiker ein hohes Interesse an Innovationsförderung haben. Denn durch die Förderung können sie Unternehmer darin unterstützen, Innovationsprojekte umzusetzen, die diese sonst nicht durchgeführt hätten. Diese Feststellung wird durch die Ergebnisse der Neuen Politischen Ökonomie, insbesondere der Theorie der politischen Konjunkturzyklen, unterstützt: Politiker haben aufgrund ihres Wiederwahlinteresses den Anreiz, diejenigen Unternehmer zu fördern, die in besonderem Maße zu Wachstum und Beschäftigung beitragen. Diese Gruppe entspricht der beschriebenen Zielgruppe der wachstumspolitischen Begründung der Innovationsförderung. (Abschnitt 4.3)

- Bezogen auf das aus Mitteln des EFRE und des Landes finanzierte Niedersächsische Innovationsförderprogramm (NIFP) bestimmen die niedersächsischen Politiker die Leitlinien der Innovationspolitik und stellen die erforderlichen Haushaltsmittel bereit, während die zuständigen Bürokraten sich um die konkrete Programmdurchsetzung kümmern. Es besteht eine Prinzipal-AgentBeziehung zwischen den Politikern als Prinzipalen und den Bürokraten als Agenten. Da die Politiker die Tätigkeit der Bürokraten nicht vollständig überwachen können, liegt eine Informationsasymmetrie („hidden action“) vor. Den zuständigen Mitarbeitern des Wirtschaftsministeriums obliegt die Auswahl der zu fördernden Unternehmen unter Berücksichtigung der Antragseva- 
luationen des Innovationszentrums, der NBank und den Innovationsberatern der Handwerkskammern. Da diese Bürokratiegruppen von der durch die Politik zugewiesenen Finanzausstattung abhängig sind, sind sie am Erfolg des NIFP interessiert. Sie unterliegen somit in unterschiedlichem Ausmaß dem Anreiz, eher risikoarme Innovationsprojekte zu fördern, welche mit einer hohen Wahrscheinlichkeit erfolgreich verlaufen und als „Leuchttürme" der regionalen Innovationspolitik vermarktet werden können. Indem die Bürokraten zur Förderung relativ sicherer Innovationsprojekte von risikoscheuen und in der Innovationstätigkeit erfahrenen Unternehmern tendieren, deckt sich ihre Zielgruppe mit der Zielgruppe der Politiker. (Abschnitte 4.3 und 4.4)

- Die empirische Evidenz der im Mai/Juni 2007 in Niedersachsen durchgeführten Unternehmensbefragung bestätigt die Hypothese, dass eher risikoscheue und in der Innovationstätigkeit erfahrene Unternehmer gefördert werden. Das NIFP erreicht also in der Praxis die durch die Politiker angestrebte Zielgruppe, da diese von den Bürokraten ausgewählt wird. (Abschnitt 6.7)

- Auch zwischen Bürokraten und Unternehmern liegt eine Prinzipal-AgentBeziehung vor, in der die Fördermittel vergebenden Bürokraten die Prinzipale und die Fördermittel empfangenden Unternehmer die Agenten sind. Die Prinzipale sind systematisch schlechter informiert als die Agenten sowohl hinsichtlich der Qualität der Projekte an sich (,hidden information“) als auch hinsichtlich der Verwendung der Fördermittel (,hidden action“). Um die erste Informationsasymmetrie zu beheben, existiert im F\&E-Programmteil des NIFP eine Qualitätskontrolle der beantragten Projekte durch das Innovationszentrum, die sicherstellen soll, dass nur besonders innovative Projekte gefördert werden. Aufgrund des kumulativen Charakters von Innovation werden diese mutmaßlich eher von Unternehmen mit hoher derzeitiger und vergangener Innovationsaktivität durchgeführt. Die empirische Evidenz bestätigt im Rahmen von uni- und multivariaten Analysen, dass geförderte Unternehmen signifikant innovativer sind als nicht geförderte Unternehmen - unabhängig von der Wahl des Innovativitätsindikators. (Abschnitte 4.5, 6.4 und 6.6)

- Um die Frage zu beantworten, warum sich nur ein Teil der potentiell für Innovationsförderprogramme in Frage kommenden Unternehmen tatsächlich um Fördermittel bewirbt, wird die Entrepreneurship-Theorie in die institutionenökonomische Analyse mit einbezogen, da sie Verhaltensweisen der jeweiligen Unternehmer anhand ihrer Fähigkeiten und Eigenschaften erklärt. (Abschnitte 5.2 und 6.7)

- Der Begriff des Unternehmers bezeichnet denjenigen, der die wesentlichen strategischen Entscheidungen des Unternehmens trifft. Der Begriff des Entre- 
preneurs bezeichnet einen Idealtypus des Unternehmers und wird - je nach Theorieansatz - über die Informationskompetenz, die Risikoeinstellung und die Innovativität des Unternehmers definiert. Diese Eigenschaften und Fähigkeiten des Unternehmers lassen sich anhand der drei Phasen der ,entrepreneurial opportunities" (EO) (Entdeckung, Bewertung und Ausbeutung) aufzeigen, wobei eine EO als Profit versprechende Möglichkeit innerhalb eines existierenden Unternehmens definiert wird. Innovation und Innovationsförderung stellen mögliche EO dar, deren drei Phasen mit Hilfe der EntrepreneurshipTheorie untersucht werden können. (Abschnitte 5.2 und 5.3)

- Verschieden ausgebildete Informationskompetenzen der Unternehmer sind ein Grund, warum sich nur ein Teil der für ein Innovationsförderprogramm in Frage kommenden Unternehmer um Fördermittel bewirbt. Unternehmer, die über eine gut ausgeprägte Informationskompetenz verfügen, nutzen verschiedene Informationsquellen und sind besonders gut über die Bereiche Innovation und Wirtschaftsförderung informiert, so dass ihnen die Suche nach und die Verarbeitung von Informationen generell leichter fällt als anderen Unternehmern. Sie entsprechen somit dem Idealtypus des Casson'schen Entrepreneurs. Die empirische Evidenz unterstützt diese theoretische Argumentation. (Abschnitte 5.4 und 6.5 )

- Ein mögliches Hemmnis, sich für Innovationsförderprogramme zu bewerben, ist der mit einer Bewerbung verbundene bürokratische Aufwand. Wird dieser Aufwand von nicht geförderten Unternehmern überschätzt, liegt unvollständige Information vor, die Unternehmer von einer Bewerbung um Fördermittel abhalten könnte. Jedoch ergibt die empirische Evidenz das gegenteilige Resultat: Nicht geförderte Unternehmer überschätzen den Bewerbungsaufwand nicht, sondern unterschätzen ihn erheblich. (Abschnitt 6.5)

- Die Risikoeinstellung des Unternehmers liefert eine weitere mögliche Erklärung für die Entscheidung einer Bewerbung für ein Innovationsförderprogramm. Risikoscheue Unternehmer nehmen eher den vergleichsweise hohen Bewerbungs- und Dokumentationsaufwand in Kauf, da der Erhalt der Fördermittel zu einer Verringerung des finanziellen Risikos für das Unternehmen führt. Weniger risikoscheue Unternehmer, die eher dem Idealtypus des Knight'schen Entrepreneurs entsprechen, suchen sich dagegen andere Finanzierungsquellen für ihre Innovationsprojekte, die mit weniger bürokratischem Aufwand verbunden sind. Die empirische Evidenz ist nicht eindeutig und weist teilweise darauf hin, dass sich risikofreudige Unternehmer seltener und stark risikoaverse Unternehmer häufiger für Innovationsförderung bewerben. (Abschnitte 5.5, 6.5 und 6.6) 
- Die empirische Evidenz bestätigt, dass innovative Unternehmer risikoscheuer sind als nicht innovativ tätige Unternehmer. Eine mögliche Erklärung für dieses Verhalten besteht darin, dass diese Unternehmer in der Regel innovative Projekte mit einem hohen Risiko bearbeiten, weswegen sie generell Investitionsprojekte und deren Risiken genau abwägen und versuchen, vorhandene Risiken zu minimieren. (Abschnitte 5.5 und 6.5)

- Geförderte Unternehmer gehören zur Zeit ihrer Förderung zwangsläufig zu den Schumpeter'schen Entrepreneuren, die sich durch ihre Innovationstätigkeit auszeichnen. Bezogen auf die Vergangenheit ist zu vermuten, dass sie ebenfalls zu den Schumpeter'schen Entrepreneuren gehörten und gerade aufgrund ihrer Innovationserfahrung eine EO wie Innovationsförderung ergreifen. Die empirische Evidenz bestätigt die theoretische Argumentation: Geförderte Unternehmer weisen eine höhere Innovationstätigkeit auf als nicht geförderte Unternehmer und entsprechen also eher dem Typus des Schumpeter'schen Entrepreneurs als nicht geförderte Unternehmer. (Abschnitte 5.6, 6.4 und 6.6)

Die in dieser Arbeit theoretisch und empirisch herausgearbeiteten systematischen Unterschiede zwischen geförderten und nicht geförderten Unternehmen zeigen, dass das NIFP nur von einer bestimmten Unternehmens- bzw. Unternehmergruppe wahrgenommen und für interessant befunden werden. Dabei handelt es sich um innovative Unternehmen aus bestimmten Branchen (Maschinen- und Anlagenbau, Elektrotechnik sowie Hochtechnologie), die von einem Unternehmer geleitet werden, der eher risikoscheu ist und Wert auf eine umfassende Meinungsbildung durch die Nutzung von verschiedenen Informationsquellen legt. Die Beschäftigtenzahl und das Unternehmensalter leisten dagegen in der multivariaten Betrachtung keinen Erklärungsbeitrag für die Differenzierung zwischen geförderten und nicht geförderten Unternehmen. Dieser beschränkte Bewerberkreis wirft die Frage auf, ob - unabhängig vom konkreten geförderten Innovationsprojekt - die Bewerber die eigentliche wirtschaftspolitische Zielgruppe der Förderung darstellen. Daran anschließend kann diskutiert werden, inwiefern sich Ausgestaltung und Vermittlung von Innovationsförderprogrammen verändern müssen. Die in dieser Arbeit dargestellten Ergebnisse beziehen sich dabei zunächst auf das NIFP. Sie können jedoch auch auf andere Innovationsförderprogramme übertragen werden, sofern diese eine verwandte institutionelle Ausgestaltung aufweisen. 


\subsection{Wirtschaftspolitische Implikationen}

\subsubsection{Zielgruppe der Förderung}

Die Ergebnisse der theoretischen und empirischen Analyse deuten darauf hin, dass Unternehmer, die sich für ein Förderprogramm wie das NIFP bewerben, risikoscheuer sind als nicht geförderte Unternehmer. Gleichzeitig zeichnen sich innovativ tätige Unternehmer durch eine signifikant höhere Risikoaversion aus als nicht innovativ tätige Unternehmer. Obwohl Innovationen im Allgemeinen mit hohen Risiken verbunden sind, werden sie also nicht zwingend von risikofreudigen Unternehmern durchgeführt, sondern eher von denjenigen Unternehmern, die sich gründlich informieren und ihre Entscheidung für oder gegen ein Innovationsprojekt genau abwägen. Hier ergibt sich wirtschaftspolitischer Handlungsbedarf im Sinne einer staatlichen Unterstützung von Innovation. Eine staatliche finanzielle Unterstützung kann zur Verwirklichung von risikoreichen Innovationsprojekten beitragen, gegen die sich insbesondere KMU ohne Fördermittel wegen des hohen Risikos entscheiden würden.

Welche Unternehmer durch das NIFP gefördert werden, hängt jedoch nicht nur davon ab, welche Unternehmer sich für das Programm bewerben, sondern auch von der durch die zuständigen Bürokraten vorgenommenen Auswahl der zu fördernden Innovationsprojekte. Im Rahmen der institutionenökonomischen Untersuchung wurde herausgearbeitet, dass die am NIFP beteiligten Bürokraten den Anreiz haben, zu einem erfolgreichen Programm beizutragen, indem eine risikoscheue Auswahl der zu fördernden Innovationsprojekte vorgenommen wird. Die so ausgewählten Projekte haben höhere Erfolgsaussichten und tragen eher zu Wachstums- und Beschäftigungseffekten bei bzw. können als technologische „Leuchttürme“ dienen. Des Weiteren weisen durch das NIFP geförderte Unternehmer eine deutlich höhere Innovationstätigkeit auf als nicht geförderte Unternehmer und entsprechen somit eher dem Schumpeter'schen Entrepreneur. Die Förderauswahl durch die in das NIFP involvierten Organisationen stellt somit eine Förderung der innovativsten Unternehmer sicher.

Geförderte Unternehmer weisen also zusammengefasst eine höhere Innovationstätigkeit auf als nicht geförderte Unternehmer und sind gleichzeitig risikoscheuer. Dieses Ergebnis bestätigt, dass das NIFP zu den wachstumspolitisch motivierten Förderprogrammen gehört, die sich eine Stärkung eher sicherer Innovationsprojekte risikoscheuer Unternehmer, die ausreichend erfahren in der Innovationstätigkeit sind, zum Ziel gesetzt haben. Eine solche „Stärkung der Starken" stellt ein weit verbreitetes Ziel in der staatlichen Innovationsförderung dar.

Wie in Kapitel 2 dargelegt, kann Innovationsförderung aus ordnungsökonomischer Sicht sowohl mit Hilfe der positiven Wachstumseffekte als auch einem vorliegenden allokativen Marktversagen begründet werden. Die Zielgruppe 
einer solchen allokationspolitisch motivierten Innovationsförderung stellen diejenigen Unternehmer dar, die bislang noch nicht oder eher wenig innovativ tätig sind und deswegen Schwierigkeiten haben, für ihre Innovationsprojekte Finanzmittel aufzutreiben. Hierunter finden sich viele kleine Unternehmen, wie in Abschnitt 2.3 erläutert wurde. Um diese Zielgruppe in ihrer Innovationstätigkeit zu ermutigen, werden - anders als beim NIFP - Programmansätze benötigt, die sich nicht an von vornherein besonders innovative Unternehmer richten. Ein derartiges Förderprogramm müsste sehr risikoreiche Innovationsprojekte von in der Innovationstätigkeit eher unerfahrenen Unternehmern unterstützen, weil diese besondere Schwierigkeiten haben, eine Finanzierung für diese Projekte zu erhalten.

Anstelle der Zuschussförderung stellt eine Vergabe von rückzahlbaren Finanzinstrumenten für die allokationspolitische Zielgruppe einen sinnvollen Ansatz dar. Bei erfolgreichem Verlauf des Innovationsprojekts müssen die geförderten Unternehmen einen Teil oder die gesamte Förderung zurückzahlen. Zum einen ist der staatliche Finanzierungsaufwand für rückzahlbare Finanzinstrumente deswegen deutlich geringer als für eine Zuschussförderung. Zum anderen ist dieses Förderinstrument für diejenigen Unternehmer möglicherweise wenig attraktiv, welche Innovationsprojekte mit sicheren privaten Erträgen vorhaben. Bei einer erfolgsabhängigen Rückzahlung, die über den Finanzierungsaufwand anderer Finanzierungsformen hinausgehen könnte, würden sich diese Unternehmer um eine alternative Finanzierungsform bemühen. Dagegen ist die beschriebene Finanzierungsform für diejenigen Unternehmer besonders attraktiv, die Innovationsprojekte mit hohen sozialen Erträgen (also positiven externen Effekten) planen und nicht sicher sind, ob und in welcher Höhe ein privater Ertrag auftritt. Sie müssen nur im Erfolgsfall eine Rückzahlung vornehmen und sichern sich für den Fall $\mathrm{ab}$, dass kein oder nur ein geringer positiver privater Ertrag auftritt. Aus gesellschaftlicher Perspektive sind diese Innovationsprojekte besonders förderungswürdig, so dass diese Ausgestaltungsform positiv zu bewerten ist.

Die Ausgestaltung des Förderinstruments als Zuschuss trägt in erheblicher Weise zur Attraktivität des Förderprogramms bei. Diese Vermutung wurde durch die Ergebnisse der Unternehmensbefragung bestätigt. Frage 20 des Fragebogens für die geförderten Unternehmer lautete: „Würden Sie sich in Zukunft wieder für das Niedersächsische Innovationsförderprogramm bewerben, wenn anstelle eines Zuschusses folgende Finanzierungsformen angeboten würden: öffentliche Beteiligung am Unternehmen, vergünstigte Darlehen, öffentliche Bürgschaft?" Im Falle einer alternativen Ausgestaltung des Förderinstrumentes würden sich deutlich weniger Unternehmer bewerben. Für ein vergünstigtes Darlehen würden sich $44,26 \%$ bewerben. Eine öffentliche Bürgschaft ist für 
$35,25 \%$ interessant, während eine öffentliche Beteiligung nur auf das Interesse von $22,13 \%$ stößt. $^{463}$

Es ist allerdings zu berücksichtigen, dass die Unternehmen diese Frage aus der komfortablen Situation eines erhaltenen Zuschusses heraus beantworten. Im Vergleich zu einem Zuschuss schneiden die alternativen Ausgestaltungsmöglichkeiten naturgemäß schlechter ab. In der Praxis würden sich einige der befragten Unternehmen trotz verneinender Aussage wahrscheinlich trotzdem für ein alternatives Finanzierungsinstrument bewerben. Dennoch deutet das Ergebnis darauf hin, dass Teile der geförderten Unternehmen entweder die staatlichen Beihilfen gar nicht so dringend benötigen und ihre Innovationsprojekte auch aus anderen Finanzierungsquellen bestreiten könnten (vgl. hierzu die Ausführungen zu den Mitnahmeeffekten in Abschnitt 4.5.1.2) oder den zu leistenden Bewerbungs- und Dokumentationsaufwand nur dann bereit sind zu tragen, wenn sie durch eine Zuschussfinanzierung eine adäquate Gegenleistung erhalten.

\subsection{2 „Förderdschungel“}

Es wurde theoretisch und empirisch herausgearbeitet, dass die Informiertheit des Unternehmers eine entscheidende Rolle bei der Bewerbung um Innovationsfördermittel spielt. Dieses de facto wirkende Selektionskriterium wäre wirtschaftspolitisch sinnvoll, sofern die Informiertheit als Signal für die Förderwürdigkeit des Unternehmens interpretiert wird. Unternehmer wenden Transaktionskosten auf, um verschiedene Informationsquellen zu nutzen und sich in der unübersichtlichen Förderlandschaft zurechtzufinden, wenn ihr Nutzen durch Förderung besonders hoch ist. Ihre Förderungswürdigkeit demonstrieren sie demnach dadurch, dass sie sich „durch den Förderdschungel gekämpft“ haben.

Es ist allerdings fraglich, inwiefern die Informationskompetenz bzw. die Informiertheit eines Unternehmers mit Innovativität und weiteren für die Förderziele relevanten Kriterien zusammenhängen. Denn obwohl für die Innovativität eine gut ausgebildete Informationskompetenz unabdingbar ist, so besitzen doch diejenigen Unternehmer, die schon in der Vergangenheit an Förderprogrammen teilgenommen haben, relative Informationsvorteile gegenüber in der Vergangenheit nicht geförderten Unternehmern. Es ist fraglich, ob sich dadurch besondere Kompetenz zur Innovationstätigkeit ergibt. Die im Rahmen der Förderprogramme verlangten Nachweise und Formulare ähneln sich, so dass der Bewerbungsaufwand eines Unternehmens bei jeder zusätzlichen Bewerbung um Fördermittel sinkt. Durch den Informationsvorsprung steigt außerdem die Wahrscheinlichkeit, dass bereits geförderte Unternehmen erneut gefördert werden unabhängig von ihrer tatsächlichen Förderwürdigkeit.

463 Keine Angabe machten bezogen auf die Antwort „,vergünstigstes Darlehen“ 9,84\%, bezogen auf die Antwort ,öffentliche Bürgschaft“ 11,48\% und bezogen auf die Antwort „öffentliche Beteiligung“ 8,20 \%. 
Diese Argumentation wird durch das Ergebnis gestützt, dass Unternehmen, die Fördermittel aus dem NIFP erhalten bzw. sich dafür beworben haben, in den letzten drei Jahren außerdem deutlich häufiger Fördermittel aus anderen Förderprogrammen erhalten bzw. sich dafür beworben haben als die restlichen befragten Unternehmen, wie aus Tabelle 17 deutlich wird. Nähere Informationen über die Art der Förderung und der geförderten Projekte liegen nicht vor. Jedoch weist dieses Ergebnis darauf hin, dass Unternehmer, die herausgefunden haben, wie sie sich Informationen über die Förderlandschaft beschaffen können und wie die Antragstellung funktioniert, in Zukunft weitere Bewerbungen um Fördermittel in Angriff nehmen.

Tabelle 17: Teilnahme an Förderprogrammen ${ }^{464}$

\begin{tabular}{lcc}
\hline & $\begin{array}{c}\text { NIFP (gefördert oder } \\
\text { beworben) }\end{array}$ & $\begin{array}{c}\text { Restliche Unternehmen } \\
\text { der Stichprobe }\end{array}$ \\
\hline Niedersächsischer Innovationskredit & $5,26 \%$ & $0,00 \%$ \\
Andere niedersächsische & $27,05 \%$ & $12,50 \%$ \\
Förderprogramme & $27,87 \%$ & $11,67 \%$ \\
Förderprogramme von Bund/KfW & $15,57 \%$ & $5,83 \%$ \\
Förderprogramme der EU & & \\
\hline
\end{tabular}

Quelle: Eigene Auswertung.

In der Vergangenheit geförderte Unternehmer besitzen also Informationsvorteile und können ihre Informationskompetenz in Bezug auf Förderprogramme immer weiter ausbauen, während die Möglichkeit besteht, dass die unübersichtlich erscheinende Förderlandschaft eine abschreckende Wirkung auf bislang noch nicht geförderte, aber eigentlich förderwürdige Unternehmer entfaltet. Deswegen bewerben sich diese Unternehmer nicht um Fördermittel, können aber ihre Innovationsprojekte ohne Förderung eventuell nicht durchführen. Da diese Innovationsprojekte möglicherweise einen höheren Nutzen für die Volkswirtschaft generieren als die tatsächlich geförderten Projekte der besser informierten Unternehmer, besteht Notwendigkeit für eine Veränderung im Fördersystem hin zu einer größeren Transparenz, so dass die Informationsanforderungen an die potentiellen Bewerber sinken. Es ist anzunehmen, dass sich in einem sehr transparenten Fördersystem insgesamt mehr Unternehmer um Fördermittel bewerben würden, so dass die Auswahl der zu fördernden Unternehmer aus einem größeren Bewerberpool getroffen werden kann. Diese Auswahl wäre mindestens ebenso gut und tendenziell besser als die beschränkte Auswahl in einem stark intransparenten Fördersystem.

464 Die Tabelle ist nicht kumulativ interpretierbar, da ein Teil der Unternehmen von mehreren der aufgeführten Förderprogramme Mittel erhalten hat. 
Die Erhöhung der Transparenz der Förderlandschaft könnte durch eine $\mathrm{Zu}$ sammenlegung vorhandener Förderangebote und somit eine Reduzierung der Zahl der Förderprogramme erfolgen. Problematisch daran dürfte es sein, die zuständigen Organisationen zur Abgabe von Kompetenzen zu bewegen. Aufgrund des in Abschnitt 3.3.1 beschriebenen Interesses der Bürokratie an einer Budgetmaximierung und Anhäufung von Aufgaben besteht Grund zur Annahme, dass die Abgabe von Aufgaben an andere Institutionen oder Bürokratien zu Widerstand innerhalb der betroffenen Bürokratie führen würde. Hinzu kommt die besondere Schwierigkeit, eine Einigung über eine Reform des Fördersystems quasi als „Lichtung des Förderdschungels“ über verschiedene politische Ebenen hinweg zu erreichen.

\subsubsection{Beratungsangebote in der Förderlandschaft}

$\mathrm{Da}$ es in der Praxis eher unwahrscheinlich ist, dass eine nachhaltige Vereinfachung der Förderlandschaft erfolgt, ist die Rolle der Beratungsinstitutionen umso wichtiger anzusehen. Die Vielzahl an verfügbaren Förderprogrammen bedingt eine große Zahl an in die Innovationspolitik involvierten Akteuren. In Niedersachsen zählen zu diesen neben den zuständigen Ministerien z. B. das Innovationszentrum, die Innovationsberater der Kammern, die NBank, die kommunalen Wirtschaftsförderer, die Landesinitiativen der Schwerpunkttechnologiefelder und die Kompetenzzentren an den Hochschulen. Die Unübersichtlichkeit der Akteursstruktur in der niedersächsischen Technologie- und Innovationslandschaft wird sogar von der sich zu über $40 \%$ im Eigentum des Landes Niedersachsen befindlichen Nord-LB konstatiert. ${ }^{465}$ Hinzu kommen die Akteure der Innovationsförderung auf Bundes- und EU-Ebene. Mit der Vielzahl an Förderprogrammen geht also eine Vielzahl an beteiligten Akteuren und zwangsläufig auch Informationsangeboten über die verschiedenen Förderprogramme einher. Unternehmen, die sich über Förderangebote informieren wollen, nutzen zusätzlich Unternehmensnetzwerke (z. B. das Innovationsnetzwerk Niedersachsen) sowie die Internetangebote des Landes (z. B. die Internetseite der NBank ${ }^{466}$ ) und des Bundes (z. B. die Förderdatenbank ${ }^{467}$ ). Ein Teil der Unternehmen nimmt außerdem eine kostenpflichtige Beratung privater Dienstleistungsunternehmen in Anspruch.

Die Stärkung von öffentlichen Beratungsinstitutionen für den Bereich der Wirtschaftsförderung stellt einen möglichen Ansatzpunkt dar, um Unternehmen verschiedener Größen und Hintergründe die Möglichkeit zu bieten, sich kostenlos über verfügbare Fördermöglichkeiten zu informieren und somit ähnliche Ausgangsmöglichkeiten für alle Unternehmen zu schaffen. $\mathrm{Zu}$ diesem Zweck

465 Vgl. Nord-LB Regionalwirtschaft (2007), S. 23 f.

$466 \mathrm{Vgl}$. http://www.nbank.de, zugegriffen am 11.08.2008.

467 Vgl. http://www.foerderdatenbank.de, zugegriffen am 11.08.2008. 
müsste der Bekanntheitsgrad der öffentlichen Beratungsinstitutionen steigen, so dass die Unternehmen einen klaren Ansprechpartner haben. Die Innovationsberater der Handwerkskammern stellen im Rahmen des NIFP ein gutes Beispiel für solche Beratungsinstitutionen dar, da die Organisation der Kammern allen Unternehmen bekannt und zugänglich ist. Die Abwicklung der gesamten Innovations- und Technologieförderung erfolgt in Niedersachsen über die NBank, so dass hier bereits eine Zentralisierung erfolgt ist.

Die empirische Untersuchung ergab, dass ein erheblicher Teil der geförderten Unternehmen kostenpflichtige Hilfe bei der Bewerbung und Antragstellung in Anspruch nahm. Zwar zeigen die Unternehmen mit der Inanspruchnahme dieser Hilfe, dass ihnen etwas an einer Förderung liegt. Jedoch kann es nicht das Ziel der aus öffentlichen Mitteln finanzierten Wirtschaftsförderung sein, durch mangelnde Transparenz und hohe Informationsanforderungen zur Erhaltung des privaten Beratungssektors beizutragen und indirekt deren Kosten zu tragen. Die Kosten, die den Unternehmen durch die private Beratung entstehen, hoffen sie durch die Fördermittel wieder zu erlangen. Entstünden keine Beratungskosten, könnte also die staatliche Förderung sinken und die Unternehmen erhielten netto dieselbe Summe für ihr eigentliches Innovationsprojekt. Die Fördermittel könnten also sehr viel effizienter eingesetzt werden.

\subsubsection{Bewerbungsaufwand um Innovationsförderung}

Im Bereich der Innovationsförderung existieren neben der Problematik der unüberschaubaren Förderlandschaft außerdem Informationsdefizite bezüglich des für eine solche Bewerbung erforderlichen Aufwands. Obwohl das NIFP im Vergleich zu anderen Förderprogrammen überschaubare formale Anforderungen an die Bewerbungen stellt, ${ }^{468}$ so hat die empirische Analyse gezeigt, dass ein erheblicher Zeitaufwand der zuständigen Unternehmensmitarbeiter mit Antragstellung und Dokumentation der Fördermittelverwendung verbunden ist. Dieser Zeitaufwand wird von den in der Förderung unerfahrenen Unternehmern stark unterschätzt, obwohl sie die Antragstellung im Allgemeinen für aufwendig und den Ablauf von Förderung für bürokratisch halten. Demzufolge scheuen nicht geförderte Unternehmer den Bewerbungsaufwand nicht etwa deswegen, weil sie eine überzogene Vorstellung vom mit einer Förderung verbundenen organisatorischen Aufwand haben. Würden sie den tatsächlichen Aufwand kennen, so kann vermutet werden, dass ihre potentielle Bewerbungsbereitschaft weiter sinken würde. Dieser Aspekt ist insbesondere für kleine Unternehmen relevant, in denen der Unternehmer die Antragstellung nicht delegieren kann, sondern neben der allgemeinen Geschäftstätigkeit durchführt. ${ }^{469}$ Hier sollte genauer geprüft

468 Vgl. Lahner und Müller (2004), S. 36 f., für diese Einschätzung bezogen auf das NIFP/Handwerk.

469 Vgl. Lahner und Müller (2004), S. 36, bezogen auf das NIFP/Handwerk. 
werden, inwiefern Antragstellung und Dokumentation vereinfacht werden können. Dabei besteht ein "trade-off" zwischen Aufwand für das Unternehmen und besseren Beurteilungsmöglichkeiten für die zuständigen Bürokraten, inwieweit eine Förderung von Projekt und Unternehmen gerechtfertigt ist.

\subsubsection{Institutionelle Ausgestaltung des Programmablaufs}

Die vorherigen Abschnitte beziehen sich zum einen auf die Auswahl der zu fördernden Unternehmer begründet in der Ausprägung bestimmter Unternehmenscharakteristika und zum anderen auf die Entscheidung der Unternehmer, sich über Fördermöglichkeiten zu informieren und sich daran anschließend für ein passendes Förderprogramm zu bewerben. In diesem Abschnitt geht es nun um die institutionelle Ausgestaltung des NIFP und die daraus resultierenden Implikationen für die Auswahl der zu fördernden Unternehmer. Diese Auswahl erfolgt im NIFP/F\&E unter Beteiligung des Innovationszentrums, welches keine direkte Beratung der Unternehmer bei der Erstellung von Projektskizze und -antrag übernimmt. Es gibt aber auch andere organisatorische Ansätze in der Wirtschaftsförderung, bei denen die Beratung der Unternehmen, die Bewertung der Anträge und die Entscheidung über die Vergabe der Fördermittel in einer Hand gebündelt sind. So wird für das NIFP/Handwerk die technologische Komponente von den Innovationsberatern der Handwerkskammern bewertet. In anderen Bundesländern gibt es ebenfalls vergleichbare Innovationsförderprogramme, bei denen die beschriebenen Kompetenzen in einer Hand liegen. ${ }^{470}$ Diese Bündelung der Kompetenzen kann durchaus sinnvoll sein, wenn es sich um weniger komplexe technische Zusammenhänge handelt, da die Verwaltungskosten des Programms, bestehend aus Kosten für die ministeriumsexternen Gutachter und Transaktionskosten für die komplizierter werdende Entscheidungsfindung, sinken. Die Mittelvergabe kann schneller und unkomplizierter erfolgen. Dennoch besteht bei einer derartigen Konstruktion die Gefahr, dass persönliche Beziehungen der Berater zu den Antrag stellenden Unternehmern in die Fördermittelvergabe mit einfließen. So könnten die Berater Unternehmer, die ihnen gut bekannt sind, weil sie beispielsweise häufig an Kammerveranstaltungen teilnehmen oder eine hohe wirtschaftliche Bedeutung für die Region haben, bei der Fördermittelvergabe bewusst oder unbewusst bevorzugen.

Des Weiteren besteht für das NIFP wie für jedes Wirtschaftsförderprogramm die Gefahr, dass die zuständigen Bürokraten zur Förderung der erstbesten zur Antragstellung gelangenden Projekte tendieren, um das Förderbudget so schnell wie möglich auszuschöpfen. Die empirische Evidenz für das NIFP ist hier nicht eindeutig: Das NFIP/F\&E hat keine Probleme mit einer zu schnellen

470 Vgl. z. B. das Innovationsprogramm für betriebliche Forschung und Entwicklung (FuEInnovationsprogramm) in Bremen und Bremerhaven, in: http://www.big-bremen.de/de/ foerderprogramme, zugegriffen am 13.02.2008. 
Mittelausschöpfung, während das NIFP/Handwerk im Jahr 2008 voraussichtlich keine Projekte fördern kann, weil die gesamten Mittel des Jahres 2008 schon Ende 2007 verplant wurden. Um den Anreiz für die Beteiligten zu mindern, die erstbesten Projekte zu fördern, stellt ein Wettbewerb um Fördermittel generell ein sinnvolles Vergabeverfahren dar. Der Wettbewerb kann als Entdeckungsmechanismus für die Auswahl der qualitativ besten Projekte genutzt werden. $\mathrm{Zu}$ diesem Zweck werden bestimmte Bewerbungstermine festgelegt und alle eingegangenen Projektanträge miteinander verglichen, um die festgelegte Fördersumme eines Termins auf die besten Projekte aufzuteilen. Der Nachteil dieses Verfahrens besteht darin, dass für viele Unternehmen mehr Zeit zwischen Antragstellung und Förderbestätigung vergeht als in einem Verfahren ohne feste Bewerbungstermine. Vor Erhalt des Förderbescheids dürfen die Unternehmen in der Regel noch nicht mit dem Innovationsprojekt anfangen, es sei denn, sie erhalten eine Ausnahmegenehmigung. Das NIFP/Handwerk besitzt eigentlich solche festen Bewerbungstermine, so dass hier der Wettbewerb um Fördermittel mit seinen beschriebenen positiven Effekten ausgenutzt wird. Inwiefern die Mittelausschöpfung für das Jahr 2008 in einer unzureichenden Ausstattung des Programmteils mit Fördermitteln oder einer mangelnden Koordination der Beteiligten begründet liegt, kann mit den vorliegenden Informationen nicht geklärt werden.

\subsubsection{Erfolgskontrolle des Programms}

Zumal das NIFP auch in der EFRE-Förderperiode 2007-2013 fortgeführt wird, stellt sich abschließend die Frage nach dem bisherigen Erfolg des Programms. Die Zufriedenheit der geförderten Unternehmer mit dem NIFP ist jedenfalls sehr hoch. Auf die Frage, ob sich die geförderten Unternehmen in Zukunft wieder für dieses Programm bewerben würden, antworteten $86,07 \%$ mit Ja, 4,92\% mit Nein und 9,02\% machten keine Angabe.

Inwiefern das NIFP aber die von der Politik verfolgten Ziele - Erhöhung des Wirtschaftswachstums und der Beschäftigung in Niedersachsen - erreicht hat, kann im Rahmen einer institutionenökonomischen Analyse nicht beantwortet werden, da diese ein Instrument zur Untersuchung der Anreizstrukturen eines bestehenden Systems ist. Hierzu wäre eine ausführliche Evaluation des Programms unter Verwendung der von der NBank bei Antragstellung gesammelten Daten, einer aktuellen Erhebung der Daten der in der Vergangenheit geförderten Unternehmen und eventuell einem Vergleich mit nicht geförderten Unternehmen vonnöten, auch wenn letzterer mit den in Abschnitt 1.2 diskutierten Schwierigkeiten behaftet ist. Die Evaluation sollte von einer unabhängigen, nicht in den Prozess des NIFP eingebundenen Institution durchgeführt werden. Eine solche Institution kennt zwar den Programmablauf nicht so detailliert wie die involvierten Akteure, kann jedoch einen unvoreingenommenen Blick auf das Programm 
werfen. ${ }^{471}$ Neben den direkten finanziellen Effekten der Förderung sollten auch die schwierig zu quantifizierenden, jedoch immens wichtigen positiven Nebeneffekte des Programms in einer vollständigen Kosten-Nutzen-Betrachtung des NIFP berücksichtigt werden. So profitieren geförderte Unternehmer von Netzwerkeffekten sowie der Erhöhung von Prestige und Bekanntheitsgrad, indem der Unternehmer beispielsweise bei regionalen Wirtschaftsveranstaltungen sein Unternehmen und seine Innovationen präsentieren kann. Im Rahmen des NIFP/Handwerk nehmen neben der finanziellen Förderung die Verbesserung des Innovationsmanagements und somit die Qualifizierung der Mitarbeiter für zukünftige Innovationsprozesse eine wesentliche Rolle ein. Diese Unterstützung ist sehr sinnvoll, wenn die Hilfe sich direkt auf die Bedürfnisse des einzelnen Unternehmens bezieht. ${ }^{472}$ Und auch nicht geförderte Unternehmer profitieren von Innovationsförderprogrammen durch die in Abschnitt 2.3.1 dargestellten positiven externen Effekte der Innovationen.

Derartige Wirkungsanalysen und Kosten-Nutzen-Betrachtungen können auch bei Betrachtung anderer Förderprogramme - auf den Ergebnissen dieser Arbeit aufbauen, indem sie die Charakteristika, in denen sich geförderte und nicht geförderte Unternehmen bzw. Unternehmer voneinander unterscheiden, in ihren Untersuchungen berücksichtigen.

\subsection{Ausblick}

Wie eingangs erwähnt lassen sich die Ergebnisse dieser Arbeit auch auf andere Innovationsförderprogramme übertragen. In diesem Zusammenhang besteht weiterer Forschungsbedarf in den Bereichen Risikoaversion von Unternehmern, dem Zusammenhang zwischen Informiertheit und Innovativität sowie der Wirkungsweisen alternativer institutioneller Ausgestaltungen von Innovationsförderprogrammen. So ist zum einen für andere Innovationsförderprogramme zu überprüfen, ob ebenfalls Unterschiede in der Risikoeinstellung von geförderten und nicht geförderten Unternehmern vorliegen. Zum anderen lohnt eine detailliertere Betrachtung der Unterschiede in der Risikoeinstellung zwischen innovativen und nicht innovativen Unternehmern. Insbesondere empirische Untersuchungen der Risikoeinstellung mit Hilfe von Experimenten könnten zur Untersuchung dieser Fragestellungen weiterführende interessante Erkenntnisse hervorbringen, da Experimente die Risikoeinstellung der Befragten anhand einer realen Entscheidungssituation mit echten Auszahlungen besser abbilden können, als es im Rahmen einer schriftlichen Befragung mit einer fiktiven Entscheidungssituation möglich ist. Ein weiterer interessanter Untersuchungsgegenstand

471 Vgl. hierzu allgemein Fölster (1991), S. 52 ff. und 70 f.

472 Vgl. hierzu allgemein Fölster (1991), S. 68. 
für die zukünftige Forschung besteht darin, inwiefern Informationskompetenz bzw. Informiertheit eines Unternehmers mit Innovativität und weiteren für die Förderziele relevanten Kriterien zusammenhängen und welche Methoden zur Messung der Informationskompetenz hierbei verwendet werden können. Außerdem könnten zukünftige Untersuchungen sich mit den Fragestellungen beschäftigen, inwiefern eine unterschiedliche institutionelle Ausgestaltung von Innovationsförderprogrammen andere Unternehmergruppen zur Bewerbung anregt bzw. andere Unternehmergruppen für die Förderung ausgewählt werden. Die Erkenntnisse dieser Arbeit stellen für alle diese Untersuchungen einen geeigneten Ausgangspunkt dar. 


\section{Literaturverzeichnis}

Acs, Zoltán J.; Audretsch, David B. (2005), Entrepreneurship, Innovation and Technological Change, Discussion Papers on Entrepreneurship, Growth and Public Policy, Max Planck Institute for Research into Economic Systems, Group Entrepreneurship, Jena, Nr. 2105.

Aghion, Philippe; Howitt, Peter (1992), A Model of Growth Through Creative Destruction, in: Econometrica, Bd. 60 (2), S. 323-351.

Alesina, Alberto; Tabellini, Guido (2007), Bureaucrats or Politicians? Part I: A Single Policy Task, in: The American Economic Review, Bd. 97 (1), S. 169-179.

Arrow, Kenneth J. (1962a), The Economic Implications of Learning by Doing, in: The Review of Economic Studies, Bd. 29 (3), S. 155-173.

Arrow, Kenneth J. (1962b), Economic Welfare and the Allocation of Resources for Invention, in: Nelson, Richard R. (Hrsg.), The Rate and Direction of Inventive Activity: Economic and Social Factors, Nachdruck (1975), Princeton University Press, Princeton, S. 609-626.

Arrow, Kenneth J. (1985), The Economics of Agency, in: Pratt, John W.; Zeckhauser, Richard J. (Hrsg.), Principals and Agents: The Structure of Business, Harvard Business School Press, Boston/Mass., S. 37-51.

Aschhoff, B.; Doherr, T.; Löhlein, H.; Peters, B.; Rammer, C.; Schmidt, T.; Schubert, T.; Schiwebacher, F. (2007), Innovationsverhalten der deutschen Wirtschaft: Indikatorenbericht zur Innovationserhebung 2006, ZEW, Mannheim.

Astor, Thomas; Bucksteeg, Mathias; Pfeiffer, Iris (2006), Zukunft Handwerk! Der Beitrag des Handwerks im Innovationsprozess, Prognos Studien Innovation, Basel et al.

Audretsch, David B.; Fritsch, Michael (2003), Linking Entrepreneurship to Growth: The Case of West Germany, in: Industry and Innovation, Bd. 10 (1), S. 65-73.

Austen-Smith, David (1997), Interest groups: Money, information, and influence, in: Mueller, Dennis C. (Hrsg.), Perspectives on Public Choice: A Handbook, Cambridge University Press, Cambridge, S. 296-321.

Baron, Robert A. (2004), The cognitive perspective: a valuable tool for answering entrepreneurship's basic "why" questions, in: Journal of Business Venturing, Bd. 19 (2), S. 221-240.

Baumol, William J. (1968), Entrepreneurship in economic theory, in: The American Economic Review, Bd. 58 (2), S. 64-71.

Baumol, William J. (2004), Education for Innovation: Entrepreneurial Breakthroughs vs. Corporate Incremental Improvements, NBER Working Paper, Cambridge/Mass., Nr. 10578.

Becker, Gary S. (1983), A Theory of Competition Among Pressure Groups for Political Influence, in: The Quarterly Journal of Economics, Bd. 98 (3), S. 371-400. 
Berghoff, Hartmut (2004), Moderne Unternehmensgeschichte: Eine themen- und theorieorientierte Einfuihrung, UTB, Paderborn.

Berthold, Ursula (1967), Zur Theorie der Subventionen: Ein Beitrag zur mikroökonomischen Analyse der Subventionswirkung und ihrer wirtschaftspolitischen Beurteilung, Berner Beiträge zur Nationalökonomie, Bd. 6, Haupt, Bern.

Bhattacharya, Sudipto; Ritter, Jay R. (1983), Innovation and Communication: Signalling with Partial Disclosure, in: The Review of Economic Studies, Bd. 50 (2), S. 331-346.

Bianchi, Milo; Henrekson, Magnus (2005), Is Neoclassical Economics still Entrepreneurless?, in: Kyklos, Bd. 58 (3), S. 353-377.

Bizer, Kilian (1998), Individuelles Verhalten, Institutionen und responsives Recht, SofiaDiskussionsbeiträge zur Institutionenanalyse, Darmstadt, Nr. 98-3.

Bizer, Kilian; Führ, Martin; Barginda, Karsten; Cichorowski, Georg; Weber, Olaf; Wiek, Armin (2003), Evaluation des 3-Städte-Klimaschutzprojektes Viernheim, Lampertheim und Lorsch. Im Auftrag des Hessischen Ministeriums für Umwelt, Landwirtschaft und Forsten, Sofia-Studien zur Institutionenanalyse, Darmstadt, Nr. 03-1.

Blanchflower, David G.; Oswald, Andrew J. (1998), What Makes an Entrepreneur?, in: Journal of Labor Economics, Bd. 16 (1), S. 26-60.

Blaug, Mark (1997), Not Only an Economist: Recent Essays by Mark Blaug, Edward Elgar Publishing, Cheltenham.

Bogumil, Jörg (2004), Probleme und Perspektiven der Leistungsmessung in Politik und Verwaltung, in: Kuhlmann, Sabine; Bogumil, Jörg; Wollmann, Hellmut (Hrsg.), Leistungsmessung und -vergleich in Politik und Verwaltung. Konzepte und Praxis, Vs Verlag, Wiesbaden, S. 392-398.

Bonnet, Jean; Brau, Thomas; Cussy, Pascal (2008), The entrepreneurial decision-making: a complex choice where taste, risk, endowments, necessity, opportunity, personal traits and behaviour matter, University of Caen, University of Rennes 1, Working Paper, Series: Industrial Economics Nr. 2008-01.

Bosma, Niels; Jones, Kent; Autio, Erkko; Levie, Jonathan (2008), Global Entrepreneurship Monitor: 2007 Executive Report, in: http:/www.gemconsortium.org/download.asp?fid=644, zugegriffen am 11.08.2008.

Bottazzi, Laura; Peri, Giovanni (2007), The International Dynamics of R\&D and Innovation in the Long Run and in the Short Run, in: The Economic Journal, Bd. 11 (518), S. 486-511.

Brennan, Geoffrey; Buchanan, James M. (1980), The Power to Tax: Analytic Foundations of a Fiscal Constitution, Cambridge University Press, Cambridge.

Breton, Albert (1996), Competitive Governments: An Economic Theory of Politics and Public Finance, Cambridge University Press, Cambridge.

Breton, Albert; Wintrobe, Ronald (1982), The logic of bureaucratic conduct: An economic analysis of competition, exchange, and efficiency in private and public organizations, Cambridge University Press, Cambridge. 
Brockhoff, Klaus (1999), Forschung und Entwicklung: Planung und Kontrolle, 5. erg. u. erw. Aufl., Oldenbourg, München.

Brockmann, Heiner (2005), KMU: Neudefinition kleiner und mittlerer Unternehmen der Europäischen Kommission, in: WiSt, Bd. 1, S. 39-40.

Brusoni, Stefano; Cefis, Elena; Orsenigo, Luigi (2006), Innovate or Die? A critical review of the literature on innovation and performance, Working Paper, CESPRI (Centro di Ricerca sui Processi di Innovazione e Internazionalizzazione), Milano, Nr. 179.

Buchanan, James M. (1980), Rent Seeking and Profit Seeking, in: Buchanan, James M.; Tollison, Robert D.; Tullock, Gordon (Hrsg.), Toward a Theory of the Rent-Seeking Society, Texas A \& M University Press, College Station/Texas.

Buenstorf, Guido (2007), Creation and Pursuit of Entrepreneurial Opportunities: An Evolutionary Economics Perspective, in: Small Business Economics, Bd. 28 (4), S. 323 337.

Bundesministerium der Finanzen (2006), 20. Subventionsbericht, Bundestagsdrucksache, Nr. 16/1020, Berlin.

Bundesministerium für Bildung und Forschung (2008), Bundesbericht Forschung und Innovation 2008, Referat Innovationspolitische Querschnittsfragen, Rahmenbedingungen.

Busenitz, Lowell W.; Barney, J. B. (1997), Differences between entrepreneurs and managers in large organizations, in: Journal of Business Venturing, Bd. 12 (1), S. 9-30.

Busom, Isabel (2000), An Empirical Evaluation of the Effects of R\&D Subsidies, in: Economics of Innovation and New Technology, Bd. 9 (2), S. 111-148.

Caliendo, Marco; Fossen, Frank M.; Kritikos, Alexander S. (2008), Risk attitudes of nascent entrepreneurs - new evidence from an experimentally validated survey, in: Small Business Economics (im Erscheinen).

Callon, Michel (1994), Is Science a Public Good? Fifth Mullins Lecture, Virginia Polytechnic Institute, 23 March 1993, in: Science, Technology \& Human Values, Bd. 19 (4), S. 395-424.

Canepa, Alessandra; Stoneman, Paul (2002), Financing Constraints on Innovation: A European Cross Country Study, EIFC Working Paper, United Nations University, Maastricht, Nr. 02-11.

Cantillon, Richard (1755), Essai sur la nature du commerce en général, Nachdruck (1964), Augustus M. Kelley Publishers, New York.

Casson, Mark (1992), Entrepreneurship: A Model of Risky Innovation under Capital Constraints, in: Norman, George; La Manna, Manfred (Hrsg.), The New Industrial Economics: Recent Developments in Industrial Organization, Oligopoly and Game Theory, Edward Elgar Publishing, Cheltenham, S. 186-213.

Casson, Mark (2001), Der Unternehmer: Versuch einer historisch-theoretischen Deutung, in: Geschichte und Gesellschaft, Bd. 27 (4), S. 524-544. 
Casson, Mark (2002), Entrepreneurship, in: Liberty Fund Inc, The Concise Encyclopedia of Economics, in: http://www.econlib.org/library/Enc/Entrepreneurship.html, zugegriffen am 02.08.2008.

Casson, Mark (2003), The Entrepreneur: An Economic Theory, 2. Aufl., Edward Elgar Publishing, Cheltenham.

Casson, Mark (2005), Entrepreneurship and the theory of the firm, in: Journal of Economic Behavior \& Organization, Bd. 58 (2), S. 327-348.

Casson, Mark; Wadeson, Nigel (2007), The Discovery of Opportunities: Extending the Economic Theory of the Entrepreneur, in: Small Business Economics, Bd. 28 (4), S. 285-300.

Chaudhry, Azam; Garner, Phillip (2007), Do Governments Suppress Growth? Institutions, Rent-seeking, and Innovation Blocking in a Model of Schumpeterian Growth, in: Economics and Politics, Bd. 19 (1), S. 35-52.

Coase, Ronald H. (1937), The Nature of the Firm, in: Economica, Bd. 4 (16), S. 386-405.

Cohen, Wesley M.; Levinthal, Daniel A. (1990), Absorptive Capacity: A New Perspective on Learning and Innovation, in: Administrative Science Quarterly, Bd. 35 (1), S. 128152.

Commons, John R. (1934), Institutional Economics: its place in political economy, Bd. 1, Nachdruck (1990), Transaction Publishers, New Brunswick/New Jersey.

Companys, Yosem E.; McCullen, Jeffery S. (2007), Strategic Entrepreneurs at Work: The Nature, Discovery, and Exploitation of Entrepreneurial Opportunities, in: Small Business Economics, Bd. 28 (4), S. 301-322.

Cools, Eva; van den Broeck, Herman (2006), Hunting the Heffalump: Can Trait and Cognitive Characteristics Predict Entrepreneurial Orientation?, Working Paper, Universiteit Gent, Faculteit Economie en Bedrijfskunde, Gent, Nr. 2006/427.

Cox, Robert; Jacobsen, Harald K. (2001), The Framework for Inquiry, in: Diehl, Paul F. (Hrsg.), The Politics of Global Governance - International Organizations in an Interdependent World, 2. Aufl., Boulder, London, S. 101-116.

Cremer, Wolfgang (2007), Art. 97 EGV, in: Calliess, Christian; Ruffert, Matthias (Hrsg.), EUV/EGV: Das Verfassungsrecht der Europäischen Union mit Europäischer Grundrechtecharta, 3. Aufl., C. H. Beck, München.

Crozier, Michel (1964), The Bureaucratic Phenomenon, University of Chicago Press, Chicago.

Czarnitzki, Dirk (2001), Die Auswirkungen der Forschungs- und Technologiepolitik auf die Innovationsaktivitäten ostdeutscher Unternehmen, in: Journal of Applied Social Science Studies, Bd. 124 (4), S. 1-22.

Czarnitzki, Dirk; Fier, Andreas (2002), Do Innovation Subsidies Crowd Out Private Investment? Evidence from the German Service Sector, in: Applied Economics Quarterly (Konjunkturpolitik), Bd. 48 (1), S. 1-25. 
Dasgupta, Partha (1987), The economic theory of technology policy: an introduction, in: Dasgupta, Partha; Stoneman, Paul (Hrsg.), Economic Policy and Technological Performance, Cambridge University Press, Cambridge, S. 7-23.

Dasgupta, Partha; David, Paul (1985), Information Disclosure and the Economics of Science and Technology, C.E.P.R. Discussion Papers, London, Nr. 73.

Dasgupta, Partha; Maskin, Eric (1987), The Simple Economics of Research Portfolios, in: The Economic Journal, Bd. 97 (387), S. 581-595.

Dasgupta, Partha; Stiglitz, Joseph E. (1980), Industrial Structure and the Nature of Innovative Activity, in: The Economic Journal, Bd. 90 (358), S. 266-293.

David, Paul A.; Hall, Bronwyn H.; Toole, Andrew A. (2000), Is public R\&D a complement or substitute for private R\&D? A review of the econometric evidence, in: Research Policy, Bd. 29 (4-5), S. 497-529.

Dávila, Alberto; Pagán, José A.; Grau, Monserrat Viladrich (1999), Immigration reform, the INS, and the distribution of interior and border enforcement resources, in: Public Choice, Bd. 99 (3-4), S. 327-345.

Downs, Anthony (1967), Inside Bureaucracy, A RAND Corporation Research Study, Little, Brown and Company, Boston.

Drucker, P. F. (1985), Innovation and Entrepreneurship: Practice and Principles, Harper \& Row, New York.

Duncombe, William; Miner, Jerry; Ruggiero, John (1997), Empirical evaluation of bureaucratic models of inefficiency, in: Public Choice, Bd. 93 (1), S. 1-18.

Eckhardt, Jonathan T.; Shane, Scott A. (2003), Opportunities and Entrepreneurship, in: Journal of Management, Bd. 29 (3), S. 333-349.

Erlei, Mathias; Leschke, Martin; Sauerland, Dirk (1999), Neue Institutionenökonomik, Schäffer-Poeschel, Stuttgart.

Europäischer Rat (2000), Schlussfolgerungen des Vorsitzes, Lissabon, 23. und 24. März 2000.

Europäischer Rat (2002), Schlussfolgerungen des Vorsitzes, Barcelona, 15. und 16. März 2002.

European Commission; Eurostat (2005), Regions: Statistical Yearbook 2005, Office for Official Publications of the European Communities, Luxembourg.

Evans, David S.; Jovanovic, Boyan (1989), An Estimated Model of Entrepreneurial Choice under Liquidity Constraints, in: The Journal of Political Economy, Bd. 97 (4), S. 808 827.

Evans, David S.; Leighton, Linda S. (1989), Some Empirical Aspects of Entrepreneurship, in: The American Economic Review, Bd. 79 (3), S. 519-535.

Ewers, Hans-Jürgen; Brenck, Andreas (1992), Innovationsorientierte Regionalpolitik: Zwischenfazit eines Forschungsprogramms, in: Birg, Hedwig; Schalk, Hans Joachim (Hrsg.), Regionale und sektorale Strukturpolitik - Rainer Thoss zum 60. Geburtstag, Institut für Siedlungs- und Wohnungswesen, Münster, S. 309-341. 
Fallgatter, Michael J. (2004), Entrepreneurship: Konturen einer jungen Disziplin, in: Schmalenbachs Zeitschrift für betriebswirtschaftliche Forschung, Bd. 56 (1), S. 23-44.

Fier, Andreas (2002), Staatliche Förderung industrieller Forschung in Deutschland, ZEW Wirtschaftsanalysen, Bd. 62, Nomos-Verlag, Baden-Baden.

Fier, Andreas; Heger, Diana; Hussinger, Katrin (2005), Die Wirkungsanalyse staatlicher Förderprogramme durch den Einsatz von Matching- und Selektionsmodellen am Beispiel der Fertigungstechnik, ZEW-Discussion Paper, Mannheim, Nr. 05-09.

Fölster, Stefan (1991), The Art of Encouraging Invention: A New Approach to Government Innovation Policy, The Industrial Institute for Economic and Social Research, Stockholm.

Förderer, Klaus; Krey, Kerstin; Palme, Klaus (1998), Innovation und Mittelstand: Eine Umfrage bei 1871 mittelständischen Unternehmen, Beiträge zur Gesellschafts- und Bildungspolitik, Institut der deutschen Wirtschaft, Köln, Nr. 222.

Frenz, Walter (2007), Handbuch Europarecht, Bd. 3, Beihilfe- und Vergaberecht, Springer, Berlin, Heidelberg.

Frey, Bruno S.; Schneider, Friedrich (1978), An Empirical Study of Politico-Economic Interaction in the United States, in: The Review of Economics and Statistics, Bd. 60 (2), S. 174-183.

Fritsch, Michael; Wein, Thomas; Ewers, Hans-Jürgen (2003), Marktversagen und Wirtschaftspolitik: Mikroökonomische Grundlagen staatlichen Handelns, 5. überarb. u. erg. Aufl., Vahlen, München.

Geroski, Paul (1995), Markets for Technology: Knowledge, Innovation and Appropriability, in: Stoneman, Paul (Hrsg.), Handbook of the Economics of Innovation and Technological Change, Blackwell, Oxford, Cambridge/Mass., S. 90-131.

Gifford, Sharon (2005), Risk and Uncertainty, in: Acs, Zoltán J.; Audretsch, David B. (Hrsg.), Handbook of Entrepreneurship Research: An Interdisciplinary Survey and Introduction, Springer, New York, S. 37-53.

Gist, John R.; Hill, R. Carter (1981), The economics of choice in the allocation of Federal grants: An empirical test, in: Public Choice, Bd. 36 (1), S. 63-73.

Gläser, Joachim (2002), Staatliche Gründungsförderung: Erkenntnisse aus der Neuen Institutionenökonomie, Trierer Schriften zur Mittelstandsökonomie, Münster, Nr. 4.

Goodacre, Alan; Tonks, Ian (1995), Finance and Technological Change, in: Stoneman, Paul (Hrsg.), Handbook of the Economics of Innovation and Technological Change, Blackwell, Oxford, Cambridge/Mass., S. 298-341.

Görg, Holger; Strobl, Eric (2007), The Effect of R\&D Subsidies on Private R\&D, in: Economica, Bd. 74 (294), S. 215-234.

Götz, Volkmar; Martínez Soria, José (2007), Subventionsrecht (H.III.), in: Dauses, Manfred A. (Hrsg.), Handbuch des EU-Wirtschaftsrechts, Bd. 2, C. H. Beck, München. 
Grichnik, Dietmar (2006), Die Opportunity Map der internationalen Entrepreneurshipforschung: Zum Kern des interdisziplinären Forschungsprogramms, in: Zeitschrift für Betriebswirtschaft, Bd. 76 (12), S. 1303-1333.

Griffith, Rachel; Harrison, Rupert; Van Reenen, John (2006), How Special is the Special Relationship? Using the Impact of U.S. R\&D Spillovers on U.K. Firms as a Test of Technology Sourcing, in: The American Economic Review, Bd. 96 (5), S. 1859-1875.

Griliches, Zvi (1990), Patent Statistics as Economic Indicators: A Survey, in: Journal of Economic Literature, Bd. 28 (4), S. 1661-1707.

Griliches, Zvi (1992), The Search for R\&D Spillovers, in: The Scandinavian Journal of Economics, Bd. 94 (Supplement: Proceedings of a Symposium on Productivity Concepts and Measurement Problems: Welfare, Quality and Productivity in the Service Industries), S. 29-47.

Grossman, Gene M.; Helpman, Elhanan (1990a), Comparative Advantage and Long-Run Growth, in: The American Economic Review, Bd. 80 (4), S. 796-815.

Grossman, Gene M.; Helpman, Elhanan (1990b), Trade, Innovation, and Growth, in: The American Economic Review, Bd. 80 (2), S. 86-91.

Grossmann, Volker (2007), How to promote R\&D-based growth? Public education expenditure on scientists and engineers versus R\&D subsidies, in: Journal of Macroeconomics, Bd. 29 (4), S. 891-911.

Grupp, Hariolf (1997), Messung und Erklärung des Technischen Wandels: Grundzüge einer empirischen Industrieökonomik, Springer, Berlin, Heidelberg, New York.

Gutmann, Gernot (1988), Marktwirtschaft, in: Albers, Willi; Zottmann, Anton (Hrsg.), Handwörterbuch der Wirtschaftswissenschaft: zugleich Neuauflage des Handwörterbuchs' der Sozialwissenschaften, Fischer, Stuttgart et. al.

Hall, Bronwyn H. (2002), The Financing of Research and Development, in: Oxford Review of Economic Policy, Bd. 18 (1), S. 35-51.

Hansmeyer, Karl-Heinrich (1977), Transferzahlungen an Unternehmen (Subventionen), in: Handbuch der Finanzwissenschaft, Bd. 3. gänzl. neubearb. Aufl., 3. Aufl., Mohr, Tübingen, S. 959-996.

Hartig, Rainer (1990), Ökonomische und polit-ökonomische Aspekte des Einsatzes von Subventionen als Instrument der Wirtschaftspolitik: Eine theoretische und empirische Analyse am Beispiel des Landes Niedersachsen, in: Hübl, Lothar (Hrsg.), Beiträge zur angewandten Wirtschaftsforschung, Bd. 19, Duncker \& Humblot, Berlin.

Harzem, Kerstin (1988), Subventionen aus Sicht der Neuen Politischen Ökonomie, Deutscher Instituts-Verlag Köln.

Haucap, Justus; Hartwich, Tobias (2006), Fördert oder behindert die Beihilfenkontrolle der Europäischen Union den (System-)Wettbewerb?, in: Schäfer, Wolf (Hrsg.), Wirtschaftspolitik im Systemwettbewerb, Duncker \& Humblot, Berlin, S. 93-144.

Hauschildt, J. (1997), Innovationsmanagement, 2. Aufl., Vahlen, München. 
Haverkamp, Katarzyna (2007), Bestimmungsfaktoren der Vertragsforschung in Deutschland: Eine theoretische und ökonometrische Analyse, CeGE Schriften, Bd. 12, Peter Lang, Frankfurt am Main.

Henrekson, Magnus (2007), Entrepreneurship and Institutions, in: Comparative Labor Law \& Policy Journal, Bd. 28 (4), S. 717-742.

Heyden, Maribel (2002), Bestimmungsgründe von Existenzgründungen im Handwerk unter besonderer Berücksichtigung des sozioökonomischen Ansatzes, Dissertation, Göttingen.

Hodgson, Geoffrey M. (1998), The Approach of Institutional Economics, in: Journal of Economic Literature, Bd. 36 (1), S. 166-192.

Holmes, Thomas J.; Schmitz Jr., James A. (1990), A Theory of Entrepreneurship and Its Application to the Study of Business Transfers, in: The Journal of Political Economy, Bd. 98 (2), S. 265-294.

Holtz-Eakin, Douglas; Joulfaian, David; Rosen, Harvey S. (1994), Entrepreneurial decisions and liquidity constraints, in: RAND Journal of Economics, Bd. 25 (2), S. 334 347.

Hujer, Reinhard; Radić, Dubravko (2005), Evaluating the Impacts of Subsidies on Innovation Activities in Germany, in: Scottish Journal of Political Economy, Bd. 52 (4), S. 565-586.

Issing, Otmar (1979), Innovationsförderung und Ordnungspolitik, in: List-Forum, Bd. 10 (1), S. $33-51$.

Jaenichen, Sebastian; Steinrücken, Torsten (2007), Regulierung des Standortwettbewerbs durch Beihilfenrecht oder Subventionssteuern?, in: Schmollers Jahrbuch, Bd. 127 (3), S. $407-440$.

Jensen, Michael C.; Meckling, William H. (1976), Theory of the Firm: Managerial Behavior, Agency Costs and Ownership Structure, in: Journal of Financial Economics, Bd. 3, S. 305-360.

Jones, Charles I. (2002), Introduction to Economic Growth, 2. Aufl., Norton, New York, London.

Jungmittag, Andre (2004), Innovations, technological specialisation and economic growth in the EU, Commission, European, Economic Papers, Brussels, Nr. 199.

Jungmittag, Andre; Grupp, Hariolf (2006), Wechselwirkungen zwischen Innovations- und Wachstumsprozessen in Deutschland 1951-1999 im Vergleich zu 1850-1913, in: Jahrbücher für Nationalökonomie und Statistik, Bd. 226/2, Lucius \& Lucius, Stuttgart, S. 180-207.

Kahneman, Daniel; Lovallo, Dan (1994), Timid Choices and Bold Forecasts: A Cognitive Perspective on Risk Taking, in: Rumelt, Richard P.; Schendel, Dan E.; Teece, David J. (Hrsg.), Fundamental Issues in Strategy: A Research Agenda, Harvard Business School Press, Boston/Mass., S. 71-96.

Kaish, Stanley; Gilad, Benjamin (1991), Characteristics of opportunities search of entrepreneurs versus executives: sources, interests, general alertness, in: Journal of Business Venturing, Bd. $6(1)$, S. 45-61. 
Kets de Vries, Manfred F. R.; Miller, Danny (1986), Personality, Culture and Organization, in: Academy of Management Review, Bd. 11 (2), S. 266-279.

Keynes, John Maynard (1936), The General Theory of Employment Interest and Money, Harcourt, Brace and Company, New York.

Kihlstrom, Richard E.; Laffont, Jean-Jacques (1979), A General Equilibrium Entrepreneurial Theory of Firm Foundation Based on Risk Aversion, in: The Journal of Political Economy, Bd. 87 (4), S. 719-748.

Kirzner, Israel M. (1974), Competition and Entrepreneurship, 2. Aufl., University of Chicago Press, Chicago, London.

Kirzner, Israel M. (1979), Perception, Opportunity and Profit: Studies in the Theory of Entrepreneurship, University of Chicago Press, Chicago, London.

Klette, Tur Jakob; Møen, Jarle; Griliches, Zvi (2000), Do subsidies to commercial R\&D reduce market failures? Microeconometric evaluation studies, in: Research Policy, Bd. 29, S. 471-495.

Klevorick, Alvin K.; Levin, Richard C.; Nelson, Richard R.; Winter, Sidney G. (1995), On the sources and significance of interindustry differences in technological opportunities, in: Research Policy, Bd. 24 (2), S. 185-205.

Klodt, Henning (1995), Grundlagen der Forschungs- und Technologiepolitik, Vahlen, München.

Knight, Frank H. (1921), Risk, Uncertainty and Profit, Houghton Mifflin Company, Boston, New York.

Kommission der Europäischen Gemeinschaften (2005), Aktionsplan staatliche Beihilfen: Weniger und besser ausgerichtete staatliche Beihilfen - Roadmap zur Reform des Beihilferechts 2005-2009 (Konsultationspapier).

Kondratieff, Nikolai D. (1926), Die langen Wellen der Konjunktur, in: Archiv für Sozialwissenschaft und Sozialpolitik, Bd. 56 (3), S. 573-609.

Krahnen, Jan P.; Rieck, Christian; Theissen, Erik (1997), Messung individueller Risikoeinstellungen, CFS Working Paper, Frankfurt, Nr. 1997/03.

Krueger, Anne O. (1974), The Political Economy of the Rent-Seeking Society, in: The American Economic Review, Bd. 64 (3), S. 291-303.

Kuznets, Simon (1962), Inventive Activity : Problems of Definition and Measurement, in: National Bureau of Economic Research (Hrsg.), The Rate and Direction of Inventive Activity: Economic and Social Factors, Nachdruck (1975), Arno Press, New York, S. $19-43$.

Lach, Saul (2002), Do R\&D Subsidies Stimulate or Displace Private R\&D? Evidence from Israel, in: The Journal of Industrial Ecnomics, Bd. 50 (4), S. 369-390.

Lachenmaier, Stefan; Rottmann, Horst (2008), Employment Effects of Innovation at the Firm Level, in: Jahrbücher für Nationalökonomie und Statistik, Bd. 227 (3), S. 254 272. 
Lahner, Jörg (2004), Innovationsprozesse im Handwerk, Göttinger Handwerkswirtschaftliche Studien, Göttingen, Nr. 69.

Lahner, Jörg; Müller, Klaus (2004), Innovationen im Handwerk, Deutsches Handwerksinstitut, Seminar für Handwerkswesen an der Universität Göttingen, Göttinger Handwerkswirtschaftliche Arbeitshefte, Nr. 54.

Lambooy, Jan G. (2000), Regional Growth, Knowledge and Innovation, in: Kuklinski, Antoni; Orlowski, Witold M. (Hrsg.), The Knowledge-Based Economy: The Global Challenges of the 21st Century, Komitet Badan Naukowych, Warszawa, S. 100-113.

Landes, David S. (1999), The Wealth and Poverty of Nations: Why Some Are So Rich and Some So Poor, W. W. Norton \& Company, New York, London.

Langlois, Richard N. (2002), Schumpeter and the Obsolescence of the Entrepreneur, UConn Department of Economics Working Paper, Storrs/Connecticut, Nr. 2002-19.

Lazear, Edward P. (2005), Entrepreneurship, in: Journal of Labor Economics, Bd. 23 (4), S. 649-680.

Lederman, Daniel; Saenz, Laura (2005), Innovation and Development around the World, 1960-2000, Policy Research Working Paper, World Bank, Nr. 3774.

Leibenstein, Harvey (1966), Allocative Efficiency vs. "X-Efficiency", in: The American Economic Review, Bd. 56 (3), S. 392-415.

Lerner, Josh (1999), The Government as Venture Capitalist: The Long-Run Impact of the SBIR Program, in: The Journal of Business, Bd. 72 (3), S. 285-318.

Licht, Amir N. (2007), The Entrepreneurial Spirit and What the Law Can Do About It, in: Comparative Labor Law \& Policy Journal, Bd. 28 (4), S. 817-862.

Lindsay, Cotton M. (1976), A Theory of Government Enterprise, in: The Journal of Political Economy, Bd. 84 (5), S. 1061-1078.

Long, J. Scott; Freese, Jeremy (2001), Regression Models of Categorical Dependent Variables Using Stata, Stata Corporation, College Station/Texas.

Lucas Jr., Robert E. (1988), On the mechanics of economic development, in: Journal of Monetary Economics, Bd. 22 (1), S. 3-42.

Lückgen, Ingo; Oberschachtsiek, Dirk; Sternberg, Rolf; Wagner, Joachim (2006), Nascent Entrepreneurs in German Regions: Evidence from the Regional Entrepreneurship Monitor (REM), in: Fritsch, Michael; Schmude, Jürgen (Hrsg.), Entrepreneurship in the Region, Springer, Heidelberg, Berlin, S. 7-34.

Magri, Silvia (2007), The financing of small innovative firms: the Italian case, Banca d'Italia, Temi di discussione, Nr. 640 .

Marshall, Alfred (1920), Principles of Economics: An introductory volume, 8. Aufl., Nachdruck (1972), The Macmillan Press, London.

Martínez-Ros, Ester; Tribó, Josep A. (2000), R\&D Investments and Financial Mechanisms, in: Kuklinski, Antoni; Orlowski, Witold M. (Hrsg.), The Knowledge-Based Economy: The Global Challenges of the 21 st Century, Komitet Badań Naukowych, Warszawa, S. 261-273. 
Marx, Karl (1904), Das Kapital: Kritik der politischen Ökonomie, Buch III: Der Gesammtprocess der kapitalistischen Produktion, 2. Aufl., Otto Meissners Verlag, Hamburg.

Maurer, Hartmut (2006), Allgemeines Verwaltungsrecht, 16. überarb. u. erg. Aufl., C. H. Beck, München.

McMullen, Jeffery S.; Plummer, Lawrence A.; Acs, Zoltán J. (2007), What is an Entrepreneurial Opportunity?, in: Small Business Economics, Bd. 28 (4), S. 273-283.

Metcalfe, Stan (1995), The Economic Foundations of Technology Policy: Equilibrium and Evolutionary Perspectives, in: Stoneman, Paul (Hrsg.), Handbook of the Economics of Innovation and Technological Change, Blackwell, Oxford, Cambridge/Mass., S. 409512.

Meyer, John R. (1984), Deregulierung und die Wiedergeburt des Unternehmers, in: Bös, Dieter; Stolper, Hans-Dieter (Hrsg.), Schumpeter oder Keynes? Zur Wirtschaftspolitik der neunziger Jahre, Bd. 12, Springer-Verlag, Berlin et. al., S. 147-176.

Mill, James (1820), Essay on Government, in: The Encyclopedia Britannica, Supplement, Edinburgh.

Mill, John Stuart (1848), Principles of Political Economy with some of their Applications to Social Philosophy, Nachdruck (1961), Augustus M. Kelley Publishers, New York.

Miner, John B.; Raju, Nambury S. (2004), Risk Propensity Differences Between Managers and Entrepreneurs and Between Low- and High-Growth Entrepreneurs: A Reply in a More Conservative Vein, in: Journal of Applied Psychology, Bd. 89 (1), S. 3-13.

Moe, Terry M. (1997), The positive theory of public bureaucracy, in: Mueller, Dennis C. (Hrsg.), Perspectives on Public Choice: A Handbook, Cambridge University Press, Cambridge, S. 455-480.

Mohnen, Pierre; Palm, Franz C.; van der Loeff, Sybrand Schim ; Tiwari, Amaresh Kumar (2008), Financial Constraints and Other Obstacles: Are They a Threat to Innovation Activity?, CESifo Working Paper Series, Maastricht, Nr. 2204.

Morone, Piergiuseppe; Testa, Giuseppina (2008), Firms' Growth, Size and Innovation: an Investigation into the Italian Manufacturing Sector, in: Economics of Innovation and New Technology, Bd. 17 (4), S. 311-329.

Mueller, Dennis C. (2003), Public Choice III, Cambridge University Press, Cambridge.

Nee, Victor; Ingram, Paul (1998), Embeddedness and Beyond: Institutions, Exchange and Social Structure, in: Brinton, Mary C.; Nee, Victor (Hrsg.), The new institutionalism in sociology, Russell Sage Foundation, New York, S. 19-45.

Nelson, Richard R. (1959), The Simple Economics of Basic Scientific-Research, in: The Journal of Political Economy, Bd. 67 (3), S. 297-306.

Niedersächsische Staatskanzlei (2006), Hinweise zu Inhalt und Gestaltung von Zuwendungsrichtlinien. 
Niedersächsisches Institut für Wirtschaftsforschung (2008), Forschung, experimentelle Entwicklung und Innovationen in der niedersächsischen Wirtschaft: Analyse von ausgewählten Innovationsindikatoren für das Niedersächsische Ministerium für Wirtschaft, Arbeit und Verkehr, Hannover.

Niefert, Michaela; Metzger, Georg; Heger, Diana; Licht, Georg (2006), HightechGründungen in Deutschland: Trends und Entwicklungsperspektiven: Endbericht, Zentrum für Europäische Wirtschaftsforschung, Mannheim.

Niskanen, William A. (1994), Bureaucracy and Public Economics, Edward Elgar Publishing, Aldershot et. al.

Nord-LB Regionalwirtschaft (2007), Innovationsland Niedersachsen, Hannover, Braunschweig, Magdeburg.

Nordhaus, William D. (1975), The Political Business Cycle, in: The Review of Economic Studies, Bd. 42 (2), S. 169-190.

Nordhaus, William D. (2004), Schumpeterian Profits in the American Economy: Theory and Measurement, NBER Working Paper, Cambridge/Mass., Nr. 10433.

North, Douglass C. (1990), Institutions, Institutional Change and Economic Performance, Cambridge University Press, Cambridge.

Norton Jr., William I.; Moore, William T. (2002), Entrepreneurial Risk: Have We Been Asking the Wrong Question?, in: Small Business Economics, Bd. 18 (4), S. 281-287.

Oberender, Peter (1987), Möglichkeiten und Grenzen staatlicher Technologieförderung: Eine ordnungspolitische Analyse, in: Jahrbuch für Sozialwissenschaft, Bd. 38, Vandenhoeck \& Ruprecht, Göttingen, S. 127-146.

OECD; Eurostat (2005), Oslo Manual: Guidelines for collecting and interpreting innovation data, 3. Aufl.

Ostrom, Elinor (1986), An agenda for the study of institutions, in: Public Choice, Bd. 48 (1), S. 3-25.

Paldam, Martin (1997), Political business cycles, in: Mueller, Dennis C. (Hrsg.), Perspectives on Public Choice: A Handbook, Cambridge University Press, Cambridge, S. $342-370$.

Palich, Leslie E.; Bagby, D. Ray (1995), Using cognitive theory to explain entrepreneurial risk-taking: Challenging conventional wisdom, in: Journal of Business Venturing, Bd. 10, S. 425-438.

Parker, Simon C. (2007), Entrepreneurial learning and the existence of credit markets, in: Journal of Economic Behavior \& Organization, Bd. 62 (1), S. 37-46.

Patel, Pari; Pavitt, Keith (1995), Patterns of Technological Activity: their Measurement and Interpretation, in: Stoneman, Paul (Hrsg.), Handbook of the Economics of Innovation and Technological Change, Blackwell, Oxford, Cambridge/Mass., S. 14-51.

Pavitt, Keith (2001), Public Policies to Support Basic Research: What Can the Rest of the World Learn from US Theory and Practice? (And What They Should Not Learn), in: Industrial and Corporate Change, Bd. 10 (3), S. 761-779. 
Picot, Arnold; Dietl, Helmut (1990), Transaktionskostentheorie, in: WiSt (4), S. 178-184.

Piga, Claudio A.; Atzeni, Gianfranco E. (2007), R\&D Investment, Credit Rationing and Sample Selection, in: Bulletin of Economic Research, Bd. 59 (2), S. 149-178.

Pratt, John W.; Zeckhauser, Richard J. (1985), Principals and Agents: An Overview, in: Pratt, John W.; Zeckhauser, Richard J. (Hrsg.), Principals and Agents: The Structure of Business, Harvard Business School Press, Boston/Mass., S. 1-35.

Rammer, Christian; Binz, Hanna (2006), Zur Förderung von FuE in der Wirtschaft durch den Staat, in: Legler, Harald; Grenzmann, Christoph (Hrsg.), FuE-Aktivitäten der deutschen Wirtschaft, Stifterverband für die Deutsche Wissenschaft, Essen, S. 131147.

Richter, Rudolf; Furubotn, Eirik G. (2003), Neue Institutionenökonomik: Eine Einführung und kritische Würdigung, 3. Aufl., Mohr Siebeck, Tübingen.

Romer, Paul M. (1990), Endogenous Technological Change, in: The Journal of Political Economy, Bd. $98(5,2)$, S. 71-102.

Röpke, Wilhelm (1929), Staatsinterventionismus, in: Handwörterbuch der Staatswissenschaften, Ergänzungsband, 4. Aufl., Jena, S. 861-882.

Russell, Bertrand (1947), Macht: eine sozialkritische Studie, Europa Verlag, Zürich.

RWI (2004), Determinanten des Strukturwandels im deutschen Handwerk, Bd. II, Studie im Auftrag des Bundesministeriums für Wirtschaft und Arbeit, Essen.

Santarelli, Enrico; Vivarelli, Marco (2007), Entrepreneurship and the process of firms' entry, survival and growth, in: Industrial and Corporate Change, Bd. 16 (3), S. 455-488.

Sappington, David E. M. (1991), Incentives in Principal-Agent Relationships, in: The Journal of Economic Perspectives, Bd. 5 (2), S. 45-66.

Sarasvathy, Saras D. (2004), The questions we ask and the questions we care about: reformulating some problems in entrepreneurship research, in: Journal of Business Venturing, Bd. 19 (5), S. 707-717.

Sarasvathy, Saras D.; Simon, Herbert A.; Lave, Lester (1998), Perceiving and managing business risks: differences between entrepreneurs and bankers, in: Journal of Economic Behavior \& Organization, Bd. 33 (2), S. 207-225.

Say, Jean-Baptiste (1861), Traité d'économie politique ou simple exposition de la manière dont se forment, se distribuent et se consomment les richesses, 7. Aufl., Guillaumin, Paris.

Schienstock, Gerd (2000), Technology Policy in a Globalising Learning Economy. From Direct Technology Policy towards Conditions-Enabling Innovation Policy, in: Kuklinski, Antoni; Orlowski, Witold M. (Hrsg.), The Knowledge-Based Economy: The Global Challenges of the 21 st Century, Komitet Badań Naukowych, Warszawa, S. 20 31.

Schmidt, Manfred G. (1995), Wörterbuch zur Politik, Alfred Kröner Verlag, Stuttgart.

Schmoller, Gustav (1900), Grundriß der Allgemeinen Volkswirtschaftslehre. Erster größerer Teil, Duncker \& Humblot, Leipzig. 
Schotter, Andrew (1981), The economic theory of social institutions, Cambridge University Press, Cambridge.

Schotter, Andrew (1986), The evolution of rules, in: Langlois, Richard N. (Hrsg.), Economics as a process. Essays in the New Institutional Economics, Cambridge University Press, Cambridge, S. 117-133.

Schröder, Michael; Steiger, Max (1996), Zugang mittelständischer Unternehmen zum Kapitalmarkt, in: Ridinger, Rudolf; Steinröx, Manfred (Hrsg.), Mittelstandsförderung in der Praxis, Verlag Dr. Otto Schmidt, Köln, S. 112-135.

Schulte, Reinhard (2007), Kann man Entrepreneurship an Universitäten lehren? Überlegungen zur akademischen Ausbildung im unternehmerischen Denken und Handeln, in: Raich, Margit; Pechlaner, Harald; Hinterhuber, Hans H. (Hrsg.), Entrepreneurial Leadership: Profilierung in Theorie und Praxis, Deutscher Universitäts-Verlag, Wiesbaden, S. 257-276.

Schumpeter, Joseph (1926), Theorie der wirtschaftlichen Entwicklung: Eine Untersuchung über Unternehmergewinn, Kapital, Kredit, Zins und den Konjunkturzyklus, 2. neubearb. Aufl., Duncker \& Humblot, München, Leipzig.

Scott, John T. (2000), R\&D and Government Initiatives, in: Kuklinski, Antoni; Orlowski, Witold M. (Hrsg.), The Knowledge-Based Economy: The Global Challenges of the 21 st Century, Komitet Badań Naukowych, Warszawa, S. 62-68.

Shane, S. (2003), A General Theory of Entrepreneurship: The Individual-Opportunity Nexus, Edward Elgar Publishing, Cheltenham.

Shane, Scott A. (2000), Prior Knowledge and the Discovery of Entrepreneurial Opportunities, in: Organization Science, Bd. 11 (4), S. 448-469.

Shane, Scott A.; Venkataraman, S. (2000), The Promise of Entrepreneurship as a Field of Research, in: Academy of Management Review, Bd. 25 (1), S. 217-226.

Simon, Herbert A. (1955), A Behavioral Model of Rational Choice, in: The Quarterly Journal of Economics, Bd. 69 (1), S. 99-118.

Simon, Herbert A. (1959), Theories of Decision-Making in Economics and Behavioral Science, in: The American Economic Review, Bd. 49 (3), S. 253-283.

Simon, Herbert A. (1979), Rational Decision Making in Business Organizations, in: The American Economic Review, Bd. 69 (4), S. 493-513.

Simon, Herbert A. (2000), Bounded Rationality in Social Science: Today and Tomorrow, in: Mind and Society, Bd. 1 (1), S. 25-40.

Smith, Adam (1776), An Inquiry into the Nature and Causes of the Wealth of Nations, Nachdruck (1937), P. F. Collier \& Son Corporation, New York.

Steinrücken, Torsten; Jaenichen, Sebastian (2003), Heterogene Standortqualitäten und Signalstrategien: Ansiedlungsprämien, Werbung und kommunale Leuchtturmpolitk, in: Zeitschrift für Wirtschaftspolitik, Bd. 52 (3), S. 399-419.

Stewart Jr., Wayne H.; Roth, Philip L. (2001), Risk Propensity Differences Between Entrepreneurs and Managers: A Meta-Analytic Review, in: Journal of Applied Psychology, Bd. $86(1)$, S. 145-153. 
Stewart Jr., Wayne H.; Roth, Philip L. (2007), A Meta-Analysis of Achievement Motivation Differences between Entrepreneurs and Managers, in: Journal of Small Business Management, Bd. 45 (4), S. 401-421.

Thiel, Wolfgang (1988), Mikroökonomische Fundierung des dynamischen Innovationswettbewerbs, Dissertation, Karlsruhe.

Tollison, Robert D. (1982), Rent Seeking: A Survey, in: Kyklos, Bd. 35 (4), S. 575-602.

Tollison, Robert D. (1997), Rent seeking, in: Mueller, Dennis C. (Hrsg.), Perspectives on Public Choice: A Handbook, Cambridge University Press, Cambridge, S. 506-525.

Tutz, Gerhard (2000), Die Analyse kategorialer Daten: anwendungsorientierte Einführung in Logit-Modellierung und kategoriale Regression, Oldenbourg, München, Wien.

Tversky, Amos; Kahneman, Daniel (1986), Rational Choice and the Framing of Decisions, in: The Journal of Business, Bd. 59 (4, Part 2: The Behavioral Foundations of Economic Theory), S. S251-S278.

Unger, Brigitte; Zagler, Martin (2003), Institutional and Organizational Determinants of Product Innovations, in: Innovation: The European Journal of Social Science Research, Bd. 16 (3), S. 293-310.

van Praag, C. M.; Cramer, J. S. (2001), The Roots of Entrepreneurship and Labour Demand: Individual Ability and Low Risk Aversion, in: Economica, Bd. 68 (269), S. 45 62.

van Stel, Andre J.; Carree, Martin; Thurik, Roy (2005), The Effect of Entrepreneurial Activity on National Economic Growth, in: Small Business Economics, Bd. 24 (3), S. 311-321.

Vaubel, Roland (1991), A Public Choice View of International Organization, in: Vaubel, Roland; Willett, Thomas D. (Hrsg.), The Political Economy of International Organizations. A Public Choice Approach, Boulder, San Francisco, Oxford, S. 27-45.

Voigt, Stefan (2002), Institutionenökonomik, Wilhelm Fink Verlag, München.

von Hayek, Friedrich A. (1945), The Use of Knowledge in Society, in: The American Economic Review, Bd. 35 (4), S. 519-530.

von Mangoldt, Hans (1855), Die Lehre vom Unternehmergewinn: Ein Beitrag zur Volkswirtschaftslehre, Nachdruck (1966), Sauer und Auvermann, Frankfurt am Main.

von Mises, Ludwig (1949), Human Action: A Treatise on Economics, Yale University Press, New Haven.

von Thünen, Johann Heinrich (1875), Der isolirte Staat in Beziehung auf Landwirtschaft und Nationalökonomie: Der naturgemäße Arbeitslohn und dessen Verhältniß zum Zinsfuß und zur Landrente, Bd. 2, 3. Aufl., Wiegandt, Hempel \& Parey, Berlin.

Wallsten, Scott J. (2000), The effects of government-industry R\&D programs on private R\&D: the case of the Small Business Innovation Research program, in: RAND Journal of Economics, Bd. 31 (1), S. 82-100.

Williamson, Oliver E. (1990), Die ökonomischen Institutionen des Kapitalismus: Unternehmen, Märkte, Kooperationen, Mohr, Tübingen. 
Williamson, Oliver E. (1993), The Economic Analysis of Institutions and Organisations - in General and with Respect to Country Studies, OECD Economics Department Working Papers, Paris, Nr. 133.

Wintrobe, Ronald (1997), Modern bureaucratic theory, in: Mueller, Dennis C. (Hrsg.), Perspectives on Public Choice: A Handbook, Cambridge University Press, Cambridge, S. 429-454.

Wyckoff, Paul Gary (1990), The simple analytics of slack-maximizing bureaucracy, in: Public Choice, Bd. 67 (1), S. 35-47.

Zagler, Martin (2007), Innovation and Growth Policy, in: Wirtschaftspolitische Blätter, Bd. 4, S. 821-836.

Zeppernick, Ralf (1985), Mehr Staat oder mehr Markt? Die Forderungen nach einer neuen Industrie- und Forschungspolitik, in: Gutowski, Armin; Molitor, Bruno (Hrsg.), Hamburger Jahrbuch für Wirtschafts- und Gesellschaftspolitik, Bd. 30, Mohr, Tübingen, S. 69-84.

Zimmermann, Horst (1987), Der Mitnahmeeffekt, in: WiSt (7), S. 339-343. 


\section{Anhang}

\section{Anhang A: Gespräch mit Herrn Strunk-Lissowski (Innovations- berater der Handwerkskammer Hildesheim-Süd- niedersachsen) am 27.03.2008}

Machen Sie aktive Beratung in dem Sinne, dass Sie geeignete Unternehmen auf das Innovationsförderprogramm ansprechen?

In Beratungsgesprächen werden geeignete Unternehmen auf die entsprechenden Programme hingewiesen. Meistens kommen Unternehmer auf uns zu, die durch Meldungen in Lokalzeitungen, Verbandsmitteilungen, Informationsveranstaltungen der Handwerkskammern, oder durch Vermittlung der Kreishandwerkerschaften, der Wirtschaftsförderer und der Kollegen auf das Programm aufmerksam wurden.

Inwiefern werden Sie in die Antragstellung der Unternehmen mit eingebunden, um Hilfestellung zu leisten?

Erfahrene Innovationsberater erkennen anhand der Projektskizze, ob ein Innovationsprojekt für den Betrieb durchführbar ist. Allerdings findet meist durch die laufende Betreuung der Innovationsberater eine große Entwicklung vom ersten Entwurf zum fertigen Projektantrag statt.

Wichtige Kriterien für den Projektantrag sind:

- Finanzierung des Projektes durch das Unternehmen muss gesichert sein,

- Organisation innerhalb des Unternehmens muss stimmen,

- Stand der Technik muss dargestellt sein (in der Regel mit Hilfe einer Patentrecherche),

- Innovation muss über den Stand der Technik hinausgehen,

- die Innovation muss vom Markt her entwickelt werden.

Positiv ist die Zusammenarbeit mit Pilotanwendern. Allerdings kommt die Idee im Regelfall vom Betrieb auf Grund eines „demand-pull“-Impulses und nicht von einer wissenschaftlichen Einrichtung oder einem Berater. Wenn ein Betrieb schon ein Patent hat, wirkt sich dies in der Regel positiv auf den Projektantrag aus.

\section{Woran erkennen Sie potentiell für das Programm geeignete Unternehmer?}

Die Unternehmer müssen bereit sein, die Anforderungen des Programms zu erfüllen. Dazu gehören neben der Antragstellung und dem Nachweis der Projektkosten auch die Wartezeit bis zur Bewilligung des Projektes. Ein vorzeitiger Maßnahmebeginn ist nur mit Genehmigung möglich. Des Weiteren unterschätzen viele Unternehmer die Dauer und Komplexität von F\&E, so dass die Beratung von Innovationsberatern mit ihrer Erfahrung sehr hilfreich ist. So wurden in den letzten zehn Jahren landesweit ca. 500 Projekte in diesem Programmteil gefördert. Mit jedem interessierten Betrieb findet in der Frühphase der Antragstellung ein intensi- 
ves Gespräch statt, das meist mehrere Stunden dauert. In diesem Gespräch werden Chancen und Möglichkeiten des Projektes betriebsbezogen ausgelotet.

Woraus besteht (für Sie) die Zielgruppe von typischen Unternehmen, die für das Innovationsförderprogramm geeignet sind?

Typische für das Niedersächsische Innovationsförderprogramm geeignete Unternehmen befinden sich in der Wachstumsphase. Sie sind keine Gründer mehr, aber auch noch nicht etabliert. Des Weiteren sind Unternehmen mit einer „etablierten Innovationskultur“ für dieses Programm geeignet.

Erfolgt die Bewertung der Anträge zusammen mit allen Innovationsberatern der Handwerkskammern? Oder wird die Bewertung je nach Handwerkskammerbezirk des Antrag stellenden Unternehmens vorgenommen?

Es gibt nach jeder Bewerbungsrunde ein Treffen der Innovationsberater der niedersächsischen Handwerkskammern mit Vertretern des Heinz-Piest-Instituts für Handwerkstechnik an der Leibniz Universität Hannover, des Innovationszentrums Niedersachsen, der Vereinigung der Handwerkskammern Niedersachsen, des Wirtschaftsministeriums und der NBank. Diese Runde spricht Förderempfehlungen aus. Die NBank prüft noch die Formalia, bevor die Projekte gefördert werden können. Ca. 90 \% der Förderanträge, die es in diese Runde schaffen, werden genehmigt. Denn der Selektionsprozess erfolgt vorher. Da der Innovationsberater in die Antragstellung eingebunden wird, erkennt er meist relativ schnell, ob das Projekt für die Förderung geeignet ist. Wenn nicht, rät er dem Unternehmer von einer Antragstellung ab.

Anmerkung: Andere Bundesländer finden das Programm sehr interessant, insbesondere wegen der Bewertung der Anträge direkt durch die Innovationsberater, die im Regelfall einen tiefgehenden Einblick in die Betriebe haben.

Wie nehmen Sie die inhaltliche Bewertung der Anträge (die ja aus den verschiedensten Bereichen stammen) vor? Gibt es bestimmte festgelegte Kriterien?

Die Innovationsberater nehmen neben der technologischen und organisatorischen Bewertung auch die Bewertung der Finanzsituation der Unternehmen vor. Dabei ist es wichtig, dass die Finanzsituation der Unternehmen eine erfolgreiche Umsetzung des Projektes gewährleistet. Nur in Ausnahmefällen setzt sich der Innovationsberater mit der Hausbank in Verbindung, um die Gesamtfinanzierung des Projektes zu sichern. Bei der Bewertung der Risiken stellt das Marktrisiko ein signifikant hohes Risiko dar. Die technologischen Risiken sind im Regelfall beherrschbarer.

\section{Wie definieren Sie Innovation für das Handwerk?}

Es handelt sich hauptsächlich um Produkt-, Verfahrens- und Dienstleistungsinnovationen, und es muss sich um deutschlandweite Neuheiten handeln. Manche in der Vergangenheit geförderte Unternehmen erzielen heute 30-40\% ihres Umsatzes mit den entsprechenden innovativen Produkten. Der Gegenstand der Förderung ist durch die Richtlinie vorgegeben. 
Wird Ihre Arbeit in der Handwerkskammer evaluiert? Bzw. was ist die Zielsetzung Ihrer Arbeit, gibt es eine Erfolgsmessung? Was ist das Interesse der Handwerkskammer bei der Innovationsförderung?

Die NBank evaluiert die Projekte insbesondere in Bezug darauf, ob die Angaben der Unternehmen im Antrag sich im Nachhinein bestätigen. So werden beispielsweise die im Antrag angegebenen Beschäftigungssteigerungen überprüft. Generell ist es jedoch schwierig, die Erfolge von Innovationsprojekten zu evaluieren. Dazu müssten Größen wie der unternehmerische Umsatz aus innovativen Produkten drei Jahre nach der Förderung erhoben und untersucht werden. Eine qualitative Einschätzung der direkt betreuten Unternehmen kann von den Innovationsberatern selbst vorgenommen werden. Die Arbeit der Innovationsberater selbst wird aber nicht direkt an quantitativen Größen des Förderprogramms gemessen. Unter den geförderten Projekten sind immer mal wieder „Leuchtturmprojekte“ dabei, also Erfolgsbeispiele von geförderten Unternehmen, die dazu benutzt werden können, um Werbung für das Programm zu machen und den Programnmerfolg darzustellen.

\section{Welche Schwierigkeiten treten bei der Förderung auf?}

Bei marktnahen Projekten, die z. B. auf Anregung der Nachfrage durchgeführt werden, werden manche Unternehmer davon abgeschreckt, dass sie nur zu festen Antragsterminen die Förderung beantragen können und somit teilweise einige Zeit warten müssen, bis sie mit der Durchführung beginnen können. Probleme treten auf, wenn die Betriebe ihre Projekte nicht fristgerecht beenden können. Gründe für eine Verlängerung der Laufzeit können sein: die gute Auftragslage (oft), technische Schwierigkeiten (seltener), lange Lieferzeiten für Vorprodukte bzw. Produktionsfaktoren (manchmal) Die Förderquote eines Projektes beträgt maximal $35 \%$. Der Hochschulanteil kann mit $50 \%$ gefördert werden.

\section{Wie bewerten Sie das Innovationsförderprogramm?}

Ich bewerte das Programm sehr positiv. Insbesondere ist das Programm erfolgreich darin, den Handwerksbetrieben die Strukturierung von F\&E-Prozessen im Sinne eines erfolgreichen Projektmanagements nahezubringen, was die Handwerksbetriebe auch in ihrer zukünftigen Projektplanung (ohne Förderung) anwenden können. 


\section{Anhang B: Gespräch mit Herrn Dr. Buß (Innovationszentrum Niedersachsen) am 28.03.2008}

Wie wichtig ist der Bereich der Technologieberatung im Vergleich zur Bearbeitung der Förderanträge für das Innovationszentrum?

Die Ausrichtung als Technologiescout und strategische Beratung für Technologie- und Innovationspolitik steht für ca. $85 \%$ der Arbeit des Innovationszentrums. Die Arbeit der Bewertung der Förderanträge nimmt nur ca. $15 \%$ der Kapazität ein. Die Arbeit mit den Unternehmen und den konkreten Förderanträgen dient dem Innovationszentrum auch als gesunde Erdung und ein „Dranbleiben“ an den unternehmerischen Belangen.

Der Bereich Konzeption/Evaluation von innovationspolitischen Instrumenten beinhaltet eine wissenschaftliche Auseinandersetzung mit der Förderpolitik? Wie unabhängig ist das Innovationszentrum?

Die Innovationszentrum Niedersachsen $\mathrm{GmbH}$ sieht sich als vollkommen unabhängig an, u. a. auch deswegen, weil ihre Kosten und Mitarbeiter durch eine feste Rahmenfinanzierung gedeckt werden und nicht über einzelne Aufträge erfolgt. Die Bezeichnung als ,think tank“ ist treffend.

\section{Wie läuft die Bewertung von Förderanträgen ab? (vgl. Abbildung 10)}

Das Antrag stellende Unternehmen reicht eine Projektskizze bei der NBank ein, die „das Gesicht zum Kunden“ ist. Die NBank prüft auf formale Vollständigkeit der Skizzenunterlage. Das Innovationszentrum bewertet die Projektskizze, die im Durchschnitt ca. 5-8 Seiten beträgt. Dabei wird gegebenenfalls über die NBank Kontakt zum Unternehmen aufgenommen, z. B. verbunden mit einem Besuch beim Unternehmen, um sich selbst einen Eindruck zu verschaffen. Dies gilt insbesondere bei KMU. Das Ziel des Innovationszentrums, das meist auch verwirklicht werden kann, ist eine Bearbeitungszeit der Projektskizze von höchstens 14 Tagen, um dem Unternehmen über die NBank zügig eine erste Rückmeldung zu geben.

Vielfach werden die Gutachten des Innovationszentrums, insbesondere wenn die Skizze zur Überarbeitung zurück an den Antragsteller geht, als konstruktive Hilfestellung (z. B. Konkretisierung des Lösungsweges) wahrgenommen. Durch optionale Unternehmensbesuche und Gesprächsangebote zusammen mit der. NBank sollen insbesondere auch Unternehmen mit bisher geringer Innovationsbeteiligung und wenig Erfahrung in der Skizzenerstellung Gelegenheit gegeben werden, gute Projektideen nach Skizzeneinreichung verständlich zu präsentieren.

\section{Nach welchen Kriterien werden die Förderanträge durch das Innovationszentrum bewertet?}

Es werden eine Technologieanalyse, eine Markteinschätzung und eine Ressourcenbewertung vorgenommen, die auf dem Foliensatz näher ausgeführt werden. . Dabei betrachtet das Innovationszentrum gegebenenfalls auch die gesamtwirtschaftliche Situation des (jungen) Unter- 
nehmens, wobei jedoch die NBank die wirtschaftlichen Kennzahlen und die Finanzierung des Projektes prüft.

Wichtige Kriterien bei der Bewertung der Förderanträge sind:

- Neuheit in Deutschland

- Kennt sich ein Unternehmen inhaltlich in dem Bereich aus, für den es Förderung beantragt (Erfahrung des Managements)?

- Hat das Unternehmen schon früher F\&E betrieben?

- Ist der technische Lösungsweg ausreichend detailliert und nachvollziehbar?

- Hat das Unternehmen die notwendigen Ressourcen, um das Projekt durchzuführen? Insbesondere die Wissensressourcen spielen hier eine entscheidende Rolle. Aber auch die Frage, ob ein Unternehmen den finanziellen Eigenanteil stemmen kann, was insbesondere für Gründer ein Problem darstellen kann, die dann aber auf andere Förderprogramme zugreifen können.

- Darüber hinaus erfolgt eine Abschätzung, ob der geplante Ressourcenansatz (Personal-, Sachkosten, Fremdleistungen etc.) angemessen erscheint.

- Gibt es ausreichende Knowhow-Zugewinne und Wertschöpfungsbeiträge, die in der Region verbleiben? Liegt ein Vermarktungsinteresse des antragstellenden Unternehmens vor? Es können auch überregionale Kooperationen gefördert werden, doch es ist wichtig, dass der Großteil der Wertschöpfung in Niedersachsen bleibt.

- Im Innovationsförderprogramm sind Unternehmen, jedoch keine Hochschulen und Forschungseinrichtungen antragsberechtigt.

Innovationsprojekte sind mit zwei Risiken behaftet: dem technologischen Risiko und dem wirtschaftlichen Risiko. Beide Risiken müssen gegeben sein, damit ein Unternehmen mit niedersächsischen Steuermitteln gefördert werden kann. Das technologische Risiko bedeutet, dass die Gefahr bestehen muss, dass das Innovationsprojekt an technologischen Schwierigkeiten scheitert. Das wirtschaftliche Risiko bedeutet u. a., dass Ertragslage und Projektkosten in einem angemessen Verhältnis stehen sollten.

\section{Erfolgt eine Evaluation der Innovationspolitik durch das Innovationszentrum?}

Das Innovationszentrum evaluiert verschiedene Instrumente der Technologie- und Innovationspolitik des Landes (z. B. Landesinitiativen, Strukturen und Förderprogramme im Technologietransfer etc.) Die eigentliche Datenbank-Dokumentation, das Berichtswesen und die Abwicklung des Innovationsförderprogramms läuft bei der NBank, mit der zusammen regelmäßig Auswertungen erfolgen. Dabei findet bisher jedoch noch keine (methodisch nicht ganz einfach zu fassende) statistische Wirkanalyse (z. B. Regressionsanalysen und Vergleichgruppenanalysen) statt. Daneben gibt es regelmäßig Studien des Niedersächsischen Instituts für Wirtschaftsforschung zur F\&E-Dynamik in Niedersachsen.

Welchen wissenschaftlichen Hintergrund haben die Mitarbeiter des Innovationszentrums (eher die technische oder die wirtschaftliche Seite)?

Die Mitarbeiter, die die Förderanträge bewerten, kommen aus den unterschiedlichsten wissenschaftlichen Disziplinen (Agrarwirtschaft, Luft- und Raumfahrttechnik, Maschinenbau etc.). Die Förderanträge werden je nach Sachgebiet auf die Mitarbeiter aufgeteilt. Oftmals wird ergänzend eine Technologieeinschätzung bei den zuständigen Landesinitiativen angefragt, 
aber das endgültige Gutachten wird vom Innovationszentrum geschrieben und vom $\mathrm{Ge}$ schäftsführer freigegeben.

Wer evaluiert die Arbeit des Innovationszentrums?

Alle drei bis vier Monate wird der Aufsichtsrat, in dem die vier Landesministerien Umwelt, Landwirtschaft, Wirtschaft und Finanzen vertreten sind, über die Arbeit des Innovationszentrums informiert. Der Aufsichtsrat ist bislang mit der Arbeit des Innovationszentrums sehr zufrieden. Das Innovationszentrum wurde noch von der Vorgänger-Regierung eingerichtet und arbeitet parteiunabhängig. Auch die jetzige Regierung zeigt sich mit der Arbeit des Innovationszentrums sehr zufrieden. 


\title{
Anhang C: Fragebogen für durch das NIFP geförderte Unter- nehmen
}

\author{
Q \\ GEORG-AUGUST-UNIVERSITÄT \\ GÖTTINGEN
}
Dipl.-Volksw. Verena Mertins Volkswirtschaftuiches Seminar Platz der Gottinger Sieben 3, 37073 Gottingen Tel: $0551-397400$, Fax: 0551-3919558 E-Mal: vmertingondg de

\section{Befragung zur Innovationsförderung in Niedersachsen}

Ihre Mitarbeit ist auberst wichtig, Sie können damit entscheidend zur Verbesserung der Ausgestaltung und des Ablaufs der Niedersäachsischen Inrovationsoorderung beitragen.

Das Ausinllen des Fragebogens dauort 10-15 Minuten. Bitle beantworten Sie möglichst alle Fragen. Thre Angaben werden selbstverständlich vertraulich twhandelt. Die anschließende Auswertung crfolgt anonym.

Unter dem Begriff Innovation sollen in diesem Fragebogen verstanden werden: (1) neue oder wesentlich verbesserte Produkte oder Dienstleistungen (Produktimnovationen), die Sie am Markt einfuhren, und (2) neue oder wesentlich verbesserte Fertigungs- Verfahrenstechniken (Prozessimnovationen), die Sie in Threm Untemehmen einführen.

\section{Angaben zu Ihrem Unternehmen}

1) Welche Funktion haben Sie im Unternehmen?

2) In welcher Branche ist Jhr Lnternehnen tätig?

3) Wie lange existiert Ihr Intemehmen schon? Seit Jahren.

4) Wie lauten die ersten beiden Zahlen der Postleitzahl lhres Lntemehmenssitzes?

5) Ist lhr Lintemehmen Mitglied in einer Industrie- und Handelskanmer?

口 Ja $\square$ Nein

Ist lhr Unternehmen Mitglied in einer Handwerkskammer?

$\square \mathrm{Ja}$ a Nein

6) Wie viele Beschaftigte hat lhr Lntemehmen insgesant?

Wie viele Beschäftigte arbeiten im Bereich F\&E bzw. Innovation?

7) Hat Thr Untemehmen eine eigene Abteilung, die forr Forschung und Entwicklung bzw. fur den Bereich Innovation zuständig ist? $\square \mathrm{Ja}$ Nein

8) Wie viele Patente besitzt Ihr Untemehmen?

9) Hat thr Lintemehmen in den Jahren 2004-2006 neue oder merklich ver- $\square$ Ja Dein besserte Produkte'Dienstleistungen aut den Markt gebracht?

Hat lhr Untemehmen in den Jahren 2004-2006 untemehmensinten $\square$ Ja $\square$ Nein neue oder merklich verbesserte Fertigungs-/Verfahrenstechniken eingefithrt?

Sind in Ihrem Unternehmen in den Jahren 2004-2006 Aktivinaten, die $\square \mathrm{Ja}$ Nein die Entwicklung oder Einfühnung von Produkt- bzw. Prozessinnovationen eum Ziel hatlen, noch nichl abgeschlossen worden?

Sind in threm Unternehmen in den Jahren 2004-2006 Aktivitälen, die dic Entwicklung oder Einfuhrung von Produk1- bzw. Prozessimnovationen zum Ziel hatten, ganz abgebrochen worden? 
10) Innovationsaktivitäten verursachen sine Vielzahl von Aufwenchungen (z.B. Erwerb von l.izenzen, Produktdesign. Weiterbildung der Mitarbeiter usw.), dic uber die reinen F\&F.Autwendungen hinausgelhen.

Schătzen Sie bitte den Anteil der Innovationsaufwendungen (laufende Autwendungen und Investitionen) am (Imsatz im Jahr 2006: $\%$

11) Wie hoch ist der durchschnittliche Anteil Thres Unternehmensumsalzes mit Marktneuhaiten sowie merklich verbesserten Produkten bzw. Dienstleistungen am Gesamtumsatz? \%

\section{Informationsquellen}

12) Welche Bedeutung haben folgende Informationsquelken für Thr Linternehmen, ...

\begin{tabular}{|c|c|c|}
\hline & $\begin{array}{l}\text {... um sich uber Wirt- } \\
\text { schaftsförderprogramme } \\
\text { zu informieren? }\end{array}$ & $\begin{array}{l}\text {... um Anrcgungen für } \\
\text { Innovationen zu } \\
\text { crhalten? }\end{array}$ \\
\hline & 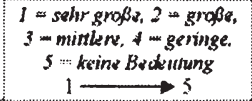 & $\begin{array}{c}1=\text { sehr gro } \beta, 2 \text { as großs, } \\
3-\text { mittlere, } 4-\text { geringe. } \\
5=\text { keine Beckutung } \\
1 \longrightarrow 5\end{array}$ \\
\hline $\begin{array}{l}\text { Informationsschriften/Internctseite des } \\
\text { L.andes Niedersachsen und der NBank }\end{array}$ & ロロロロロ & ロロロロ \\
\hline BMW i-Forderdutenbunk & ロロロロロ & 00000 \\
\hline $\begin{array}{l}\text { Kommunale Wirtschafts fordereinrich- } \\
\text { ungen }\end{array}$ & ロロロロロ & 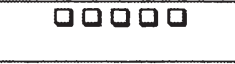 \\
\hline Innovations herater der Kammern & ロロロロ & ロロロロロ \\
\hline Verblinde & ロロロロロ & ロロロロロ \\
\hline Kunden, Licferanten, Hausbanken & ロロロロロ & ロロロロロ \\
\hline Internet, Presse, Rundfunk, Femsehen & ロロロロロ & ロロロロロ \\
\hline Patentdatenbanken & ロロロQם & ロロロロ \\
\hline Hochschultransfercinrichtungen & ロロロロロ & ロロロロロ \\
\hline Fachmessen, Fachtagungen & ロロロロ & ロロロロ \\
\hline Andere Betriebe & ロロロロ & ㅁㅁ \\
\hline Unternchmensnetzwerke & वロव口व & ロロロロロ \\
\hline Private Unternehmensbcrater & ロロロロ & ロロロロ \\
\hline Aus dem Unternelimen heraus & ロロロロロ & ロロロロ \\
\hline Sonstiges: & ロロロロ & ロロロロ \\
\hline
\end{tabular}

13) Gibt es eine bestimmte Person bzw. Personen, die in Ihrem Lntemehmen für die Suche nach Wirtschaftsförderprogrammen verantwortlich istisind?

Ja. Fs handelt sich um (Funktion im (intemehmen):

Dein. 
14) Welche Bedeutung haben folgende Innovationshemmunisse tür Ihr Unternehmen?

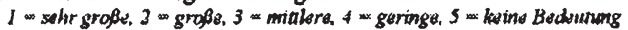

\begin{tabular}{|c|c|}
\hline Innovationsrisiko & ロロロロ \\
\hline Mangel an Eigen-oder Fremdkapilal & 00000 \\
\hline Mangel an geeignetem Fachpersonal & $0 \square \square \square \square$ \\
\hline Unternehmensinterne Widerstände & 口व口व \\
\hline Mangel an Informationen (Technologie, Marketing, usw.) & $0 \square 0 \square \square$ \\
\hline Mangel an geeigneten Kooperationspartnern & 口व口व \\
\hline Gesetzgetung, Regelungen & 口ロロ口 \\
\hline Sonstiges: & 口ロロロ \\
\hline
\end{tabular}

\section{Fragen zur Person}

15) Welche der folgenden Eigenschaften schätzen Sie als wichtig ein um ein erfolgreicher Lintemehmor zu sein?

$l=$ außerordontlich wichtig, $2 m$ sehr wichtig, $3=$ wichtig, 4 wenigor wichtig, $5=$ wnwichbg

\begin{tabular}{|c|c|}
\hline Risikobereituchan & 口םव口 \\
\hline gut informicrt scin & ロロロロ口 \\
\hline Aufgeschlossenheit gegenubber Neuem & ㅁㅁㅁ \\
\hline Entscheidungsfahigkeit & $0 \square \square \square \square$ \\
\hline Koondinations - und Organisationstahigkeit & ロロםロ \\
\hline Frindungsgeist/Kreativitat & ロロロ口 \\
\hline Sonstige Eigenxchuften: & ם वם \\
\hline
\end{tabular}

16) Wie schătzen Sie persönlich die folgenden zwei Investitionsmöglichkeiten ein?

Moglichkeit A: Sie erhalten als lirtrag absolut sicher $100.000 \mathrm{E}$.

Moglichkeit B: Sie erhalten als Ertrag nit einer Wahrscheinlichkeit von $50 \%$ einen bestimmen Geldbetrag in Höhe von X.

Wie hoch müsste dieser (ieldbetrag $X$ sein, damit Sie beide Investitionsmöglichkeiten vollkommen gleich bewenten?
Q Kleiner als $190.000 E$
Q Zwischen 210.000 und $250.000 €$
a zwischen 190.000 und $210.000 €$
口 Grober als $250.000 €$

\section{Teilnahme am Niedersåchsischen Innovationsförderprogramm}

17) For welche dieser niedersachsischen Programme hat sich Ihr Untemehmen in den letzten drei Jahren theworben und aus welchen Programmen hat Thr Unternehmon in den letzten drei Jahren Fordennittel erhalten? (Mehrfachnenmungen moglich)

$\square$ Niedersăchsisches lnnovationsforderprogramm

a) fur Forschungs- und Entwicklungsausgaben

b) tür das Handwerk

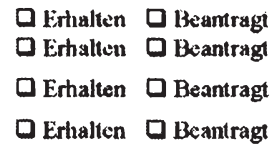

Q Niedersächsischer Innovationskredit

口 Andere niedersāchsische Wirtschaftsförderprogramme:

Erhalien Beantragt 
Hat sich Ihr Unternehmen in den letalen drei Jahren für Wirtsclaftsförderprograume des Bundes, der KfW oder der EU beworben und aus welchen Programmen hat Ihr Unternehmen in den letzten drei Jahren Fördermittel erhalten? (Mehrfachnennungen moglich)

Wirtschaftsförderprogramme von Bund bzw. KfW: $\square$ Eirhahen $\square$ Beantragt

Wirtschaftsförderprogramme der EU: Q Erhalten Deantragt

18) W(irden Sie sich in Zukunf wieder fìr das Niedersăchsische Innovationsförderprogramm bewerben? $\square$ Ja Nein

19) Welcke der folgenden Aussagen trelfen Ihrer Ansicht nach auf das Nieckrsächsische Innovationsforderprogramm zu?
Die Antragstellung ist
aufwendig
nicht aufwendig
Der Ablauf der Förderung verlief
$\square$ bürokratisch
$\square$ unbürokratisch
Die Fördersummen sind
zu gering
$\square$ ausreichend
Die Förderlandschaft ist
$\square$ unabersichtlich
$\square$ abersichllich

Sonstige Anmerkıngen zu Ablauf und Ausgestaltung:

20) Würden Sic sich in Zukunft wieder für das Niedersä̈hsische Inwvationsförderprogramm bewerben, wann anstelle cines Zuschusses folgende Finauzierungsformen angeboten werden:
a) öflentliche Beteiligung am Unternehmen
○ Ja Nein
b) vergunstigte Darlehen
a Ja Nein
c) Sfrentiche Burgschat
$\square \mathrm{Ja} \square$ Nein

21) Schätzen Sie bitte. welcher Zeitaufwand in Ihnem Unternehmen benötigt wurde. um im Rahmen des Niedersächsischen Inuюvationsförderprogranuns ...

- die Antragskizze zu erstellen: ca. Persomenstunden,

- den Forderantrag zu arstellen: ca. Personenstunden.

- die Dokumentation der Fördermitteherwendung zu erstellen (falls bereits erfolgt): ca. Personenstunden.

22) Hahen Sie exteme Hilfe bei der Antragstellung in Anspruch genommen?

a Ja. Die exteme Hilfe hat ca. Euro gekostet. $\square$ Nein.

23) Angenommen, lhr Untemehmen hätte keine Fördermittel durch das Niederstichsische Innovationsforderprogramm erhalten. Ware das Innovationsprojekt trotzdem durchgefuhrt worden?

Wir hätten das Innovationsprojekt allch ohne Förderung durchgefuhrt.

$\square$ Wir luatten nur eine abgespeckte Version durchgefülurt im I mfang von ca. $\%$ der urspränglich veranschlagten Kosten.

Wir hätten das lmovationsprojekt vine Förderung garantiert nicht durchgeführt.

\section{Vielen Dank für Inre Mitarbeit!}




\section{Anhang D: Fragebogen für die Vergleichsgruppe}

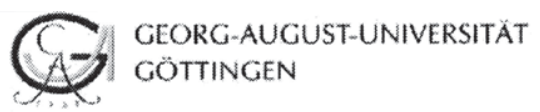

Dipd. Volksw. Verena Mertins Platz der Göttinger Sieben 3, 37073 Göttingen Tet: :0551-397400, Fax: 0551-3919558

E-Mail: vmertinggudg de

\section{Befragung: Innovationsverhalten niedersächsischer Unternehmen}

Ihre Mitarbeit ist außerst wichtig. Das Ausfallen des Fragebogens dauert 10-15 Minuten. Bitte beantworten Sie möglichst alle Fragen. Ihre Angaben wenden selbstverständlich vertraulich behandelt. Die anschliebende Auswertung erfolgt anonym.

Unter dem Begriff Imnovation sollen in diesem Fragebogen verstanden werden: (1) neue oder wesentlich verbesserte Produkte oder Dienstleistungen (Produdtinnovationen), die Sie sm Markt einführen, und (2) neue oder wesentlich verbesserte Fertigungs-/Verfahrenstechniken (Prozessinnovationen), die Sie in Ihrem Unternehmen einführen.

\section{Angaben zu Ihrem Unternehmen}

1) Welche Funktion haben Sie im Intemehmen?

2) In welcher Branche ist Ihr L'nternehmen tätig?

3) Wie lange existier Ihr Internehmen schon? Seit Jahrell.

4) Wie lauten die ersten beiden Zahlen der Postleitzahl Ihres Unternehmenssitzes?

5) Ist Ihr Intemehmen Mitglied in eilher Industrie- und Handelskammer? $\square \mathrm{Ja} \square$ Niein Ist Jhr I internetmmen Mitglied in einer Handwerkskammer? $\square$ Ja $\square$ Nein

6) Wis vicle Beschåftigte hat Ihr Lintemehmen insgesant?

Wie viele Beschäftigte arteiten im Bercich F\&E bzw. Innovation?

7) Hat lhr Ǔnternehmen eine eigene Abteilung, die für Forschung und Fntwicklung bzw. für den Bereich Innuvation zuständig ist? $\square \mathrm{Ja} \square$ Nein

8) Wie viele Patente besitz thr Untemehmen?

9) Hat lhr Unternehmen in den Jahren 2004-2006 neuc oder merklich ver. $\square \mathrm{Ja}$ Q Niein besserte Produkte Dienstleistungen auf den Markt gebracht?

Hat llur Unternehmen in den Jahren 2004-2006 untemehmensintern $\square$ Ja $\square$ Nein neue oder merklich verbesserte Fertigungs-l'erfahrenstechniken eingelührt?

Sind in Ihrem Untemehmen in den Jahren 2004-2006. Aktivitaten, dic $\mathrm{Ja}$ Nein dic Entwicklung oder Einfuhrung von Produkt- bzw. Prozessinnovationen num Z.iel hatten, moch nicht abgeschlossen worden?

Sind in llurem Untemehmen in den Jahren 2004-2006 Aktivitalen. die die Entwicklung oder Einfahnung von Produkt- bzw. Prozessinnovationen zum Ziel hallen, ganz abgebrochen worden? 
10) Innovationsaktivităten verursachen eine Vielzahl von Aufivenchungen (z.B. Enwerb von Lizenzen, Produltdesign, Weiterbildung der Mitarbeiter usw.), die ūber die reinen F\&LAufwendungen hinausgehen.

Schătzen Sie bitte den Anteil der lmnovationsaufwendungen (laufende Aufivendungen und Investitionen) am Umsatz im Jahr 2006: $\%$

11) Wie hoch ist der durchschnittiche Amteil thres tintemehmensumsatzes mit Marktneuheiten sowie merklich verbesserten Produkten baw. Dienstleistungen am Gesamtunsatz? $\%$

\section{Informationsquellen}

12) Welche Bedeutung haben folgende Informationsquellen fur thr Unternetumen, ...

\begin{tabular}{|c|c|c|}
\hline & $\begin{array}{l}\text {... um sich uber Wint. } \\
\text { schafisforderyprogramme } \\
\text { zu infonnieren? }\end{array}$ & $\begin{array}{l}\text {... un Anregungen für } \\
\text { Innevationen zu } \\
\text { erhalton? }\end{array}$ \\
\hline & 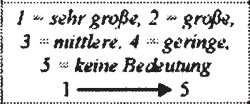 & 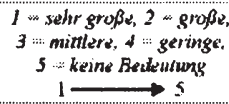 \\
\hline $\begin{array}{l}\text { Informationsschriften Internetseite des } \\
\text { I andes Nicdersachsen und der NBank }\end{array}$ & ロロロロロ & ロロロロロ \\
\hline BMW i-Forderdatenbank & 00000 & 00000 \\
\hline $\begin{array}{l}\text { Kommunale Wirtschaftsfordereintich- } \\
\text { tungen }\end{array}$ & ロロロロ & םロロロ \\
\hline Innovationsberater der Kammern & 00000 & वववव \\
\hline Verhande & םםロ & םםロ \\
\hline Kunden, Lieferanten, Hausbanken & ロロロロ & ロロロロロ \\
\hline Internet, Presse. Rundfunk, Fernsehen & ロロロロ & ロ \\
\hline Patentdatenhanken & םロ & ロ \\
\hline Hochschultransfercinrichtungen & घロव口व & ロロロロ \\
\hline Fachmessen, Fachtagungen & ロ & פםロ \\
\hline Andere Betriebe & ロロロロ & ㅁㅁㅁ \\
\hline Unternehmensnetzwerke & पव口व & ㅁㅁㅁ \\
\hline Privale Unternehmensberaler & ロロロロ & ロロロロロ \\
\hline Aus dem Unternelimen heraus & ㅁㅁㅁ & ㅁㅁㅁㅁ \\
\hline onsuges. & פםם - 3ם & פロ \\
\hline
\end{tabular}

13) Gibt es eine bestimmte Person bzw. Personen, die in Ihrem Unternehmen für die Suche nach Wirtschattsförderprogrammen verantwortlich istisind?

$\square$ Ja. Es handelt sich um (Funkition im Untemehmen):

Q Nein. 
14) Welche Bedeutung haben folgende Innovationshemmnisse für Mhr Unternehmen? $1=$ sehrgroße, $2=$ gnoße. $3-$ milflers, $4=$ geringe, $5=$ ksine Buckeutung

\begin{tabular}{|l|c|}
\hline Innovationsrisiko & $\square \square \square \square \square$ \\
\hline Mangel an Eigen- eder Fremdkapital & $\square \square \square \square \square$ \\
\hline Mangel an geeignetem Fachpersonal & $\square \square \square \square \square$ \\
\hline Unternehmensinterne Widerstände & $\square \square \square \square \square$ \\
\hline Mangel an Informationen (Technologie, Marketing, usw.) & $\square \square \square \square \square$ \\
\hline Mangel an gecigneten Kooperationspartncm & $\square \square \square \square \square$ \\
\hline Gesetuyebung, Regelungen & $\square \square \square \square \square$ \\
\hline Sonstiges: & $\square \square \square \square \square$ \\
\hline
\end{tabular}

\section{Fragen zur Person}

15) Wolche dor folgenden Eigenschaften schătzen Sie als wichtig ein, um ein erfolgreichor Untemehmer zu sein?

1 - aublenorchnolich wichtig, $2=$ wehr wichtig, 3 - wichtig, $4-$ weniger wichtig, 3 - unwichtig

\begin{tabular}{|l|c|}
\hline Risikobereitschaft & $\square \square \square \square \square \square$ \\
\hline gut informicrt sein & $\square \square \square \square \square$ \\
Aufgeschlossenheit gegenubber Neuem & $\square \square \square \square \square$ \\
Entscheidungsfahigkeit & $\square \square \square \square \square$ \\
\hline Koondinations- und Organisationsfähigkeit & $\square \square \square \square \square$ \\
\hline ErfindungsgeistKreativität & $\square \square \square \square \square$ \\
Sonstige Eigenschaften: & $\square \square \square \square \square$ \\
\hline
\end{tabular}

16) Wie schatzen Sie persónlich die folgenden zwei Investitionsmoglichkeiten ein?

Möglichkeit A: Sie erhalten als Frtrag absolut sicher $100.000 \mathrm{f}$.

Mosglichkeit B: Sie erhalten als Ertrag mit einer Wahrscheinlichkeit von $50 \%$ einen bestimmten Cieldbetrag in Höhe von $X$.

Wie hoch musste dieser Celdbetrag $X$ sein. damit sic heide Investitionsmõglichkeiten vollkommen gleich bewerten?

口 Kleiner als $190.000 €$

Zwischen 190.000 und $210.000 \mathrm{f}$
Z Zwischen 210.000 und $250.000 €$

D GroBer als 250.000€

\section{Teilnahme an Wirtschaftsförderprogrammen}

17) Hat sich Ihr Untemchmen in den leteten drei Jahren for sines oder mehrere der folgenden Wirtschaftsforderprogramme beworben und ggf. Fordermittel erhalien?

Niedersachsische Wirtschaftsforderprogramme:

Q Wirtschaftsiorderprogramme von Bund bzw. KfW:

W Wirtschaftsforderprogramme der F.U: $\square$ Beworben Erhalten

Beworben E Erhalten

$\square$ Beworben $\square$ lishalten

a Nein. 
18) Hat sich Ihr Untenchmen in den letaten drei Jahren speziell beim Niedersächsischen Innovationsforderprogramm (für Forschungs- und Entwicklungsausgaben oder für das Hlandwerk) beworben?

$\square$ Ja. In diesem Fall schătzen Sie bitte, welcher Zeitaufwand in Ihrem Untemehmen insgesamt benotigi wirde. um ...

- die. Antragskizze zu erstellen: ca.

- den Förderantrag zu crstellen: is. Personenstunden,

- und (falls sine Fördenung orfolgt ist) die Dokumentation der Fordermittelverwendung zu erstellen: ca. Personenstunden.

口 Nein. In diesem fall stellen Sie sich bitte vor, llhr Unternehmen wöde sich un För* derung for ein Innovationsworhaben bewerben. Schatzen Sis bitte, welcher Zeitaufwand in Ihrem Lnternehunen insges amt benötigt wärde, urn ...

- die Antrayskizze zu crstellen: ca. Personenstunden,

- den forderantrag zu enstellen: ca. Personenstunden

- und (falls eine Forderung erfolge) die Dokumentation der Fordermittelvernwenching zu arstellen: ca. Personenstunden.

19) Welche der folgenden Aussagen treffèn Ihrer Ansicht nach auf Innovationsforderprogramme allgemein zu?

Die Antragstellung ist

Der Ablauf der Fördenung verläutt

$\square$ aufwendig

bürokratisch

$\square$ nicht aufwendig

Die Fördersummen sind

a gu gering

$\checkmark$ unbürokratisch

Die Förderlandschaft ist

$\square$ unübersichtlich

$\square$ ausreichend

uubersichtlich

20) Haben Sie noch Anmerkungen zum Thema Innovation und Innovationstörderung?

Vielen Dank für Ihre Mitarbeit! 


\section{Anhang E: Wirtschaftszweige der befragten Industriebetriebe}

\section{Statistische Systematik der Wirtschaftszweige in der Europäischen Gemeinschaft}

(NACE, Revision 2) ${ }^{473}$

15 Ernährungsgewerbe

16 Tabakverarbeitung

17 Textilgewerbe

18 Bekleidungsgewerbe

19 Ledergewerbe

20 Holzgewerbe (ohne Herstellung von Möbeln)

21 Papiergewerbe

22 Verlagsgewerbe, Druckgewerbe, Vervielfältigung von bespielten Ton-, Bild- und Datenträgern

23 Kokerei, Mineralölverarbeitung, Herstellung und Verarbeitung von Spalt- und Brutstoffen

24 Herstellung von chemischen Erzeugnissen

25 Herstellung von Gummi- und Kunststoffwaren

26 Glasgewerbe, Herstellung von Keramik, Verarbeitung von Steinen und Erden

27 Metallerzeugung und -bearbeitung

28 Herstellung von Metallerzeugnissen

29 Maschinenbau

30 Herstellung von Büromaschinen, Datenverarbeitungsgeräten und -einrichtungen

31 Herstellung von Geräten der Elektrizitätserzeugung, -verteilung u.Ä.

32 Rundfunk- und Nachrichtentechnik

33 Medizin-, Mess-, Steuer- und Regelungstechnik, Optik, Herstellung von Uhren

34 Herstellung von Kraftwagen und Kraftwagenteilen

35 Sonstiger Fahrzeugbau

36 Herstellung von Möbeln, Schmuck, Musikinstrumenten, Sportgeräten, Spielwaren und sonstigen Erzeugnissen

37 Recycling

$473 \mathrm{Vgl}$. die Internetseite von Eurostat, in: http://ec.europa.eu/eurostat/ramon, zugegriffen am 11.08.2008. 


\section{Anhang F: Gewerke der befragten Handwerksbetriebe}

\section{Anlage Nr. Gewerbe der Handwerksordnung}

A 3 Zimmerer

A 13 Metallbauer

A 14 Chirurgiemechaniker

A 15 Karosserie- und Fahrzeugbauer

A 16 Feinwerkmechaniker

A 17 Zweiradmechaniker

A 18 Kälteanlagenbauer

A 19 Informationstechniker

A 20 Kraftfahrzeugtechniker

A 21 Landmaschinenmechaniker

A 24 Installateur und Heizungsbauer

A 25 Elektrotechniker

A 26 Elektromaschinenbauer

A 27 Tischler

A 28 Boots- und Schiffbauer

A 33 Augenoptiker

A 34 Hörgeräteakustiker

A 35 Orthopädietechniker

A 36 Orthopädieschuhmacher

A 37 Zahntechniker

A 40 Glasbläser und Glasapparatebauer

B1 4 Behälter- und Apparatebauer

B1 14 Modellbauer 


\section{Anhang G: Anschreiben für die Industrieunternehmen der Ver- gleichsgruppe}

\author{
C-A Georg-August-Universität \\ Göttingen
}

\author{
Wirtschaftswissenschaftliche Fakultät \\ Volkswirtschaftliches Seminar

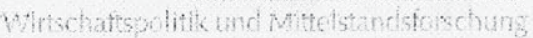

Verena Mertins · Platz der Gortinger Sieben 3 - 37073 Gơtingen

An die Geschäftsführung
Dipl.-Volksw. Verena Mertins

\section{Befragung zum Innovationsverhalten der niedersächsischen Wirtschaft}

Sehr geehrte Damen und Herren,

in Kooperation mit dem Niedersächsischen Ministerium für Wirtschaft, Arbeit und Verkehr und dem Volkswirtschaftlichen Institut für Mittelstand und Handwerk an der Universität Göttingen führen wir eine Befragung zum Innovationsverhalten der niedersăchsischen Wirtschaft durch. Dabei steht im Mittelpunkt des Forschungsvorhabens die zentrale Frage, welche Innovationshemmnisse für niedersăchsische Unternehmen von Bedeutung sind und welche Gründe Unternehmen davon abhalten, sich für Innovationsförderprogramme zu bewerben.

Wir haben Ihre Adresse von der Industrie- und Handelskammer Hannover als Teil einer Stichprobe von niedersächsischen Unternehmen erhalten. Damit die Ergebnisse dieser Evaluation repräsentativ sind und die Sichtweisen der Unternehmen angemessen berücksichtigen, sind wir unbedingt auf Ihre Mitwirkung angewiesen.

Wir bitten Sie, sich für den beigefügten Fragebogen etwas Zeit (ca. $10-15$ Minuten) zu nehmen und ihn bis zum 30.06.07 ausgefüllt im beigelegten Rückumschlag an uns zurückzusenden. Der Fragebogen sollte möglichst von einem Mitglied der Geschäftsführung ausgefüllt werden.

Ihre Angaben werden selbstverständlich von uns vertraulich behandelt. Die Nummerierung der Fragebögen erfolgt, um Unternehmen nicht erneut zu befragen, die bereits geantwortet haben. Die Auswertung der Fragebögen erfolgt vollstăndig anonym.

Năhere Informationen zum Forschungsvorhaben finden Sie auf der Homepage der Universität Göttingen: http://www.wwl.wiso.uni-goettingen.de/ehrstuhl_seite.php?ID=10\&ID_Bereich=6\&Name=Befragung

Wir bedanken uns jetzt schon für Ihre Mithilfe - insbesondere für die aufgewendete Zeit und verbleiben

mit freundlichen Grüßen 


\section{Anhang H: Anschreiben für die Handwerksunternehmen der Vergleichsgruppe}

\author{
Q \\ Georg-August-Universität \\ Göttingen
}

Wirtschaftswissenschaftliche Fakultät

Volkswirtschaftliches Seminar

Verena Mertins · Platz der Gơttinger Sieben 3 - 37073 Gottingen

An die Geschäftsführung
Dipl.-Volksw. Verena Mertins

Befragung zum Innovationsverhalten der niedersăchsischen Wirtschaft

Sehr geehrte Damen und Herren,

in Kooperation mit dem Niedersächsischen Ministerium für Wirtschaft, Arbeit und Verkehr und dem Volkswirtschaftlichen Institut für Mittelstand und Handwerk an der Universităt Göttingen führen wir eine Befragung zum Innovationsverhalten der niedersächsischen Wirtschaft durch. Dabei steht im Mittelpunkt des Forschungsvorhabens die zentrale Frage, welche Innovationshemmnisse für niedersächsische Unternehmen von Bedeutung sind und welche Gründe Unternehmen davon abhalten, sich für Innovationsförderprogramme zu bewerben.

Wir haben Ihre Adresse von der zuständigen Handwerkskammer als Teil einer Stichprobe von Unternehmen Ihrer Region erhalten. Damit die Ergebnisse dieser Evaluation repräsentativ sind und die Sichtweisen der Unternehmen angemessen berücksichtigen, sind wir unbedingt auf Ihre Mitwirkung angewiesen.

Wir bitten Sie, sich für den beigefügten Fragebogen etwas Zeit (ca. 10 -15 Minuten) zu nehmen und ihn bis zum 15.07.07 ausgefüllt im beigelegten Rückumschlag an uns zurückzusenden. Der Fragebogen sollte möglichst von einem Mitglied der Geschäftsführung ausgefullt werden.

Ihre Angaben werden selbstverständlich von uns vertraulich behandelt. Die Nummerierung der Fragebögen erfolgt, um Unternehmen nicht emeut zu befragen, die bereits geantwortet haben. Die Auswertung der Fragebögen erfolgt vollständig anonym.

Nähere Informationen zum Forschungsvorhaben finden Sie auf der Homepage der Universität Göttingen: http://uww. vwl.wiso.uni-goettingen.de/ehrstuhl_seite.php?ID=10\&ID_Bereich=6\&Name=Befragung

Wir bedanken uns jetzt schon für Ihre Mithilfe - insbesondere für die aufgewendete Zeit und verbleiben

mit freundlichen Grüßen 


\section{Anhang I: Anschreiben für durch das NIFP geförderte Unter- nehmen}

NBank Gunther-Wagner-Alles 12-14 30177 Hannover

Name des Unternehmens

Geschäftsleitung

Straße Hausnummer

Postleitzahl Ort
Hennover, 13. April 2007

Produktentwicklung / Fördercontrolling

Dr. Arnd Kölling

Telefon 0511. 30031-232

Telefax 0511. 30031-11232

and. boollingenbank.de

Innovationsförderung in Niedersachsen

Sehr geehrte Damen und Herren,

in einer Zeit die von Strukturwandel und Globalisierung geprägt ist, werden Innovationen auch für regional verwurzelte Betriebe immer bedeutsamer, um das Fortbestehen und das Wachstum der Unternehmen zu gewährleisten. Sie sind daher ein immer wichtigerer Bestandteil wirtschaftlichen Handelns in modernen Industrie- und Dienstleistungsgesellschaften. Das Land Niedersachsen hat daher die NBank damit beauftragt, Betriebe zu fördern, die Innovationen planen und durchführen möchten.

Ihr Unternehmen gehört zu denen, die vom Niedersächsischen Innovationsförderprogramm profitierten bzw. noch profitieren, da Innen zwischen 2004 und 2007 eine Förderung aus diesem Programm bewilligt wurde. Sie haben gezeigt, dass Sie unternehmerisch handeln sowie tatkräftig und zukunftsorientiert auf die Veränderungen der Wirtschaft reagieren. Wir möchten Sie nun um Ihre Mithilfe bei der Untersuchung der Innovationsförderung in Niedersachsen bitten.

Der beigefügte Fragebogen, den wir in Zusammenarbeit mit der Universität Göttingen, Professur für Wirtschaftspolitik und Mittelstandsforschung, entwickelt haben, soll die Effektivität des Niedersächsischen Innovationsförderprogramm überprüfen und helfen, die Förderung zu optimieren. Um ein genaues Bild über die Durchführung von Innovationen und die Wirkung des Förderprogramms zu bekommen, benötigen wir möglichst viele ausgefüllte Fragebögen. Daher wären wir Ihnen sehr dankbar, wenn Sie sich die Zeit nehmen würden und unsere Fragen beantworten könnten (ca. 10 - 15 Minuten). Ihre Angaben werden selbstverständlich vertraulich behandelt und nur zu dem angegebenen Zweck anonym ausgewertet. Nach Abschluss der Studie erhalten Sie eine Zusammenfassung der wichtigsten Ergebnisse.

Die Rückantwort ist für Sie natürlich kostenlos, das Porto übernehmen wir. Bitte verwenden Sie dazu den beigefügten, bereits freigemachten Rückumschlag. Wir würden uns freuen, wenn wir Ihren ausgefüllten Fragebogen bis zum \{Datum\} erhalten würden. 
Für Rückfragen stehen wir Ihnen natürlich geme zur Verfügung. Bitte wenden Sie sich an unseren Mitarbeiter Dr. Arnd Kölling (Tel.: 0511/30031-231 bzW. E-Mail: arnd.koelling@nbank.de).

Wir bedanken uns schon jetzt für Ihre Mithilfe und hoffen, dass Sie sich an unserer Untersuchung beteiligen werden.

Mit freundlichen Grüßen

Dr. Sabine Johannsen

(Vorstand)
Dr. Anja Altmann

(Leitung ProduktentwickJung / Fördercontrolling) 


\section{Anhang J}

Tabelle 18: Informationsquellen für Innovation bezogen auf nicht geförderte und durch Innovationsförderung geförderte Unternehmen (nur innovative Unternehmen)

\begin{tabular}{|c|c|c|c|c|c|c|}
\hline Informationsquellen für Innovation & $\begin{array}{l}\text { NIFP- } \\
\text { Förderung } \\
\text { (Mittelwert) }\end{array}$ & $\begin{array}{c}\text { Keine } \\
\text { Bewerbung } \\
\text { (Mittelwert) }\end{array}$ & $\begin{array}{l}\text { NIFP- } \\
\text { Förderung } \\
\text { (Median) }\end{array}$ & $\begin{array}{c}\text { Keine } \\
\text { Bewerbung } \\
\text { (Median) }\end{array}$ & $\begin{array}{l}\text { Mann-Whitney- } \\
\text { U-Prüfgröße } \\
\text { Z-Wert }\end{array}$ & $\operatorname{Pr}$ \\
\hline Aus dem Unternehmen heraus & 2,26 & 2,30 & 2 & 2 & 0,54 & 0,59 \\
\hline Fachmessen/-tagungen & 2,28 & 2,48 & 2 & 3 & 1,23 & 0,22 \\
\hline Kunden/Lieferanten/Hausbanken & 2,68 & 2,41 & 2 & 2 & $-1,07$ & 0,29 \\
\hline Andere Betriebe & 2,98 & 3,38 & 3 & 3 & 1,96 & 0,05 \\
\hline Internet/Presse/Rundfunk/Fernsehen & 3,03 & 3,20 & 3 & 3 & 0,72 & 0,47 \\
\hline Unternehmensnetzwerke & 3,36 & 3,96 & 3 & 4 & 2,87 & $0,00 * * *$ \\
\hline Hochschultransfereinrichtungen & 3,35 & 4,20 & 3 & 5 & 3,93 & $0,00 * * *$ \\
\hline Patentdatenbanken & 3,78 & 4,62 & 4 & 5 & 4,57 & $0,00 * * *$ \\
\hline Innovationsberater der Kammern & 3,74 & 4,24 & 4 & 5 & 2,57 & $0,01 * *$ \\
\hline Verbände & 3,78 & 3,61 & 4 & 4 & $-0,44$ & 0,66 \\
\hline $\begin{array}{l}\text { Informationsschriften/Internetseite } \\
\text { des Landes Niedersachsens und der } \\
\text { NBank }\end{array}$ & 3,84 & 4,33 & 4 & 5 & 3,02 & $0,00 * * *$ \\
\hline $\begin{array}{l}\text { Kommunale Wirtschaftsförder- } \\
\text { einrichtungen }\end{array}$ & 4,04 & 4,28 & 4 & 5 & 1,56 & 0,12 \\
\hline $\begin{array}{l}\text { BMWi-Förderdatenbank } \\
\text { Private Unternehmensberater }\end{array}$ & $\begin{array}{l}4,16 \\
4,36\end{array}$ & $\begin{array}{l}4,64 \\
4,56\end{array}$ & $\begin{array}{l}4 \\
5\end{array}$ & $\begin{array}{l}5 \\
5\end{array}$ & $\begin{array}{l}3,21 \\
1,38\end{array}$ & $\begin{array}{l}0,00 * * * \\
0,17\end{array}$ \\
\hline
\end{tabular}

Quelle: Eigene Auswertung. 


\section{Anhang K: Branchenzugehörigkeit und -dummies des Verarbei- tenden Gewerbes}

NACE Industriezweige des Verarbeitenden Gewerbes

Rohstoffherstellung und -verarbeitung (Branchendummy 1)

20 Holzgewerbe (ohne Herstellung von Möbeln)

21 Papiergewerbe

22 Verlagsgewerbe, Druckgewerbe, Vervielfältigung von bespielten Ton-, Bild- und Datenträgern

23 Kokerei, Mineralölverarbeitung, Herstellung und Verarbeitung von Spalt- und Brutstoffen

24 Herstellung von chemischen Erzeugnissen

25 Herstellung von Gummi- und Kunststoffwaren

26 Glasgewerbe, Herstellung von Keramik, Verarbeitung von Steinen und Erden

27 Metallerzeugung und -bearbeitung

28 Herstellung von Metallerzeugnissen

Maschinen- und Anlagenbau, Elektroindustrie (Branchendummy 2)

29 Maschinenbau

30 Herstellung von Büromaschinen, Datenverarbeitungsgeräten und -einrichtungen

31 Herstellung von Geräten der Elektrizitätserzeugung, -verteilung u. ä.

Hochtechnologie (Branchendummy 3)

32 Rundfunk- und Nachrichtentechnik

33 Medizin-, Mess-, Steuer- und Regelungstechnik, Optik, Herstellung von Uhren

34 Herstellung von Kraftwagen und Kraftwagenteilen

35 Sonstiger Fahrzeugbau

Sonstige Branchen des Verarbeitenden Gewerbes (Referenzkategorie)

15 Ernährungsgewerbe

16 Tabakverarbeitung

17 Textilgewerbe

18 Bekleidungsgewerbe

19 Ledergewerbe

36 Herstellung von Möbeln, Schmuck, Musikinstrumenten, Sportgeräten, Spielwaren und sonstigen

Erzeugnissen

37 Recycling 


\section{Anhang L: Branchenzugehörigkeit und -dummies des Handwerks}

Anlage Nr. Gewerbe der Handwerksordnung

Rohstoffherstellung und -verarbeitung (Branchendummy 1)

A 13 Metallbauer

A 40 Glasbläser und Glasapparatebauer

Maschinen- und Anlagenbau, Elektroindustrie (Branchendummy 2)

A 16 Feinwerkmechaniker

A 18 Kälteanlagenbauer

A 25 Elektrotechniker

A 26 Elektromaschinenbauer

B1 4 Behälter- und Apparatebauer

B1 14 Modellbauer

Hochtechnologie (Branchendummy 3)

A 14 Chirurgiemechaniker

A 15 Karosserie- und Fahrzeugbauer

A 28 Boots- und Schiffbauer

A 33 Augenoptiker

A 34 Hörgeräteakustiker

A 35 Orthopädietechniker

A 36 Orthopädieschuhmacher

A 37 Zahntechniker

Sonstige Branchen des Verarbeitenden Gewerbes (Referenzkategorie)

A 3 Zimmerer

A 17 Zweiradmechaniker

A 19 Informationstechniker

A 20 Kraftfahrzeugtechniker

A 21 Landmaschinenmechaniker

A 24 Installateur und Heizungsbauer

A 27 Tischler 


\section{Anhang M}

Tabelle 19: Ergebnisse des Logit-Modells nur für innovativ tätige Unternehmen

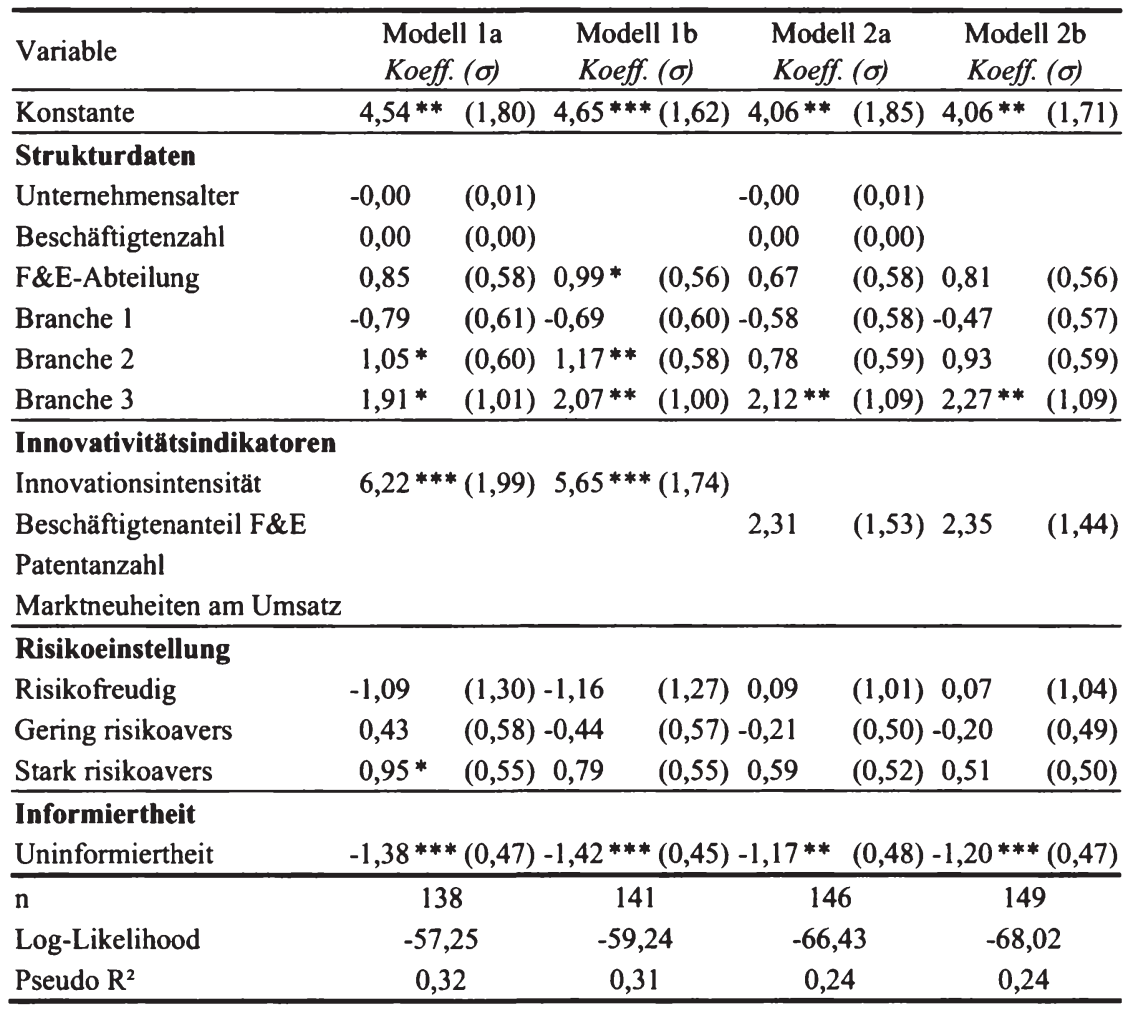

Huber-White Standardfehler in Klammern, *10\%,**5\%,*** $1 \%$, \# Variable wurde aus dem Logit herausgenommen wegen vollständiger Vorhersehbarkeit des Erfolgs.

Quelle: Eigene Auswertung. 
Tabelle 19: Ergebnisse des Logit-Modells nur für innovativ tätige Unternehmen (Forts.)

\begin{tabular}{|c|c|c|c|c|c|c|c|c|}
\hline \multirow{2}{*}{$\begin{array}{l}\text { Variable } \\
\text { Konstante }\end{array}$} & \multicolumn{2}{|c|}{$\begin{array}{l}\text { Modell 3a } \\
\text { Koeff. }(\sigma)\end{array}$} & \multicolumn{2}{|c|}{$\begin{array}{l}\text { Modell 3b } \\
\text { Koeff. }(\sigma)\end{array}$} & \multicolumn{2}{|c|}{$\begin{array}{l}\text { Modell 4a } \\
\text { Koeff. }(\sigma)\end{array}$} & \multicolumn{2}{|c|}{$\begin{array}{l}\text { Modell } 4 \mathrm{~b} \\
\text { Koeff. }(\sigma)\end{array}$} \\
\hline & $5,02 * *$ & $*(1,70)$ & $4,75 * * *$ & $(1,63)$ & $4,21 * *$ & $(1,88)$ & $4,25 * *$ & $(1,74)$ \\
\hline \multicolumn{9}{|l|}{ Strukturdaten } \\
\hline Unternehmensalter & $-0,01$ & $(0,01)$ & & & $-0,00$ & $(0,01)$ & & \\
\hline Beschäftigtenzahl & $-0,00$ & $(0,00)$ & & & 0,00 & $(0,00)$ & & \\
\hline F\&E-Abteilung & 0,69 & $(0,58)$ & 0,78 & $(0,57)$ & 0,31 & $(0,58)$ & 0,49 & $(0,57)$ \\
\hline Branche 1 & $-1,08 *$ & $(0,57)$ & $-1,21 * *$ & $(0,54)$ & $-0,82$ & $(0,57)$ & $-0,71$ & $(0,56)$ \\
\hline Branche 2 & 0,16 & $(0,54)$ & 0,17 & $(0,51)$ & 0,61 & $(0,55)$ & 0,79 & $(0,55)$ \\
\hline Branche 3 & \# & & \# & & $2,04^{*}$ & $(1,14)$ & $2,16^{*}$ & $(1,13)$ \\
\hline
\end{tabular}

\section{Innovativitătsindikatoren}

Innovationsintensität

Beschäftigtenanteil F\&E

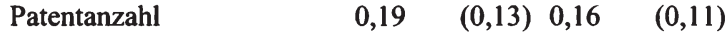

Marktneuheiten am Umsatz

$2,83 * *(1,13) 2,71 * * *(1,04)$

\begin{tabular}{|c|c|c|c|c|c|c|c|c|}
\hline \multicolumn{9}{|l|}{ Risikoeinstellung } \\
\hline Risikofreudig & 0,14 & $(1,13)$ & 0,23 & $(1,16)$ & 0,16 & $(1,37)$ & 0,11 & $(1,38)$ \\
\hline Gering risikoavers & $-0,14$ & $(0,54)$ & $-0,16$ & $(0,53)$ & $-0,30$ & $(0,54)$ & $-0,30$ & $(0,54)$ \\
\hline Stark risikoavers & 0,58 & $(0,54)$ & 0,54 & $(0,53)$ & 0,54 & $(0,54)$ & 0,45 & $(0,53)$ \\
\hline \multicolumn{9}{|l|}{ Informiertheit } \\
\hline Uninformiertheit & \multicolumn{8}{|c|}{$-1,19 * * *(0,45)-1,20 * * *(0,45)-1,20 * *$} \\
\hline$n$ & \multicolumn{2}{|c|}{124} & \multicolumn{2}{|c|}{126} & \multicolumn{2}{|c|}{137} & \multicolumn{2}{|c|}{140} \\
\hline Log-Likelihood & \multicolumn{2}{|c|}{$-63,38$} & \multicolumn{2}{|c|}{$-65,55$} & \multicolumn{2}{|c|}{$-61,99$} & \multicolumn{2}{|c|}{$-63,60$} \\
\hline Pseudo $\mathrm{R}^{2}$ & \multicolumn{2}{|c|}{0,19} & \multicolumn{2}{|c|}{0,18} & \multicolumn{2}{|c|}{0,25} & \multicolumn{2}{|c|}{0,25} \\
\hline
\end{tabular}


Das cege - Centrum für Europa-, Govemance- und Entwicklungsforschung - wurde 1999 von der Wirtschaftswissenschafttichen Fakultăt der Georg-August-Universitắt gegründet. Das cege dient als Forum zur internationalen und interdisziplinären Forschungszusammenarbeit in den Themenbereichen Europäische Integration, Governance und Entwicklungsökonomik. In den cege-Schriften werden Forschungsergebnisse aus Dissertationen, Habilitationen oder anderen Forschungsprojekten des cege veröffentlicht.

Band 1 Axel Gerloff: Wechselkurspolitik in Mittel- und Osteuropa. Eine theoretische Analyse unter besonderer Berücksichtigung der Erfahrungen der zehn Kandidaten für eine EU-Osterweiterung. 2001.

Band 2 Ingo Konrad: Zur Integration ausgewăhlter mittel- und osteuropăischer Lănder in die wăhrungspolitische Ordnung Europas. 2002.

Band 3 Axel Hennighausen: Wegekostenfinanzlerung und Lenkung im deregulierten europåischen Verkehrsmarkt. 2002.

Band 4 Wolfgang Münch: Effects of EU Enlargement to the Central European Countries on Agricultural Markets. 2002.

Band 5 Holger Niermann: Welchen Finanzausgleich braucht Europa? Ein konkreter Vorschlag zur Neugestaltung der europäischen Finanzbezlehungen mit Simulationsmodell. 2002.

Band 6 Jens Südekum: Agglomeration and Regional Unemployment Disparities. A Theoretical Analysis with Reference to the European Union. 2003.

Band 7 Christoph Schinke: Der Geldmarkt im Euro-Wăhrungsraum. Geldmarktgeschăfte, Zinsbildung und die Taylor Rule. 2004.

Band 8 Kai Stukenbrock: The Stability of Currency Boards. 2004.

Band 9 Harald Grethe: Effects of Including Agricultural Products in the Customs Union between Turkey and the EU. A Partial Equilibrium Analysis for Turkey. 2004.

Band 10 Gơtz Zeddies: Perspektiven der Gemeinschaftspolitiken in der erweiterten EU. 2005.

Band 11 Ingmar Kumpmann: Systemwettbewerb und Umverteilung. Gefăhrdet die Globalisierung den Sozialstaat? 2005.

Band 12 Katarzyna Haverkamp: Bestimmungsfaktoren der Vertragsforschung in Deutschland. Eine theoretische und Okonometrische Analyse. 2007.

Band 13 Peter Schwarz: Kapitalsteuerwettbewerb zwischen Nationalstaaten unter besonderer Berücksichtigung Deutschlands. Eine empirische Analyse. 2007.

Band 14 Andreas Poppe: Auswirkungen der Einführung einer konsolidierten Körperschaftsteuer-Bemessungsgrundlage in der Europăischen Union. Eine empirische Analyse der Konzernstrukturen und des Steueraufkommens. 2008.

Band 15 Sebastian Hess: Meta-Analyse angewandter Gleichgewichtsmodelle des internationalen Agrarhandels. 2008.

Band 16 Mareike Köller: Ausländische Direktinvestitionen und regionale Integration - das Beispiel Irland. 2009.

Band 17 Verena Mertins: Institutionenőkonomische Analyse von Innovationsförderung. Eine theoretische und empirische Betrachtung am Beispiel Nledersachsens. 2009.

www.peterlang.de 